\title{
Cancer Metastases of 202 Kinds of Cancers via Cytocapsular Tubes
}

Tingfang $\mathrm{Yi}^{1,2,3 *}, \mathrm{PhD}$, Gerhard Wagner ${ }^{1,2,3,4}, \mathrm{PhD}$

1. Cytocapsula Research Institute, 245 First Street, Cambridge, MA, 02142 USA

2. Cellmig Biolabs Inc., 790 Memorial Drive, Cambridge, MA, 02139 USA

3. Celldim Therapeutics Inc., 790 Memorial Drive, Cambridge, MA, 02139 USA

4. Department of Biological Chemistry and Molecular Pharmacology of Harvard Medical School, 240 Longwood, Boston, MA, 02115 USA

*Address reprint requests to Dr. Tingfang Yi, Cytocapsula Research Institute, 245 First Street, Cambridge, MA, 02142 USA, or at tyi@ cytocapsula.com.

BACKGROUND Cancer metastasis is the primary source of solid cancer lethality, but the underlying mechanisms have essentially remained elusive. Recently, we found that cytocapsular tubes, a newly discovered organelle of mammalian cells, conduct cell translocation, suggesting an efficient pathway for cancer cell metastases.

METHODS We performed immunohistochemistry staining and fluorescence microscope imaging analyses of 6 kinds of normal organs ( $n=14$ patients), 38 subtypes of benign tumors ( $\mathrm{n}=$ 126 patients), and 8,061 clinical solid cancer tissue samples (covering 202 types and subtypes of cancers, and including cancers, paracancer tissues and metastatic cancers) taken from 7,125 cancer patients. These solid cancers cover 30 types of organs. We characterized cytocapsular tubes (CTs), cell migration in CTs, and CT quantity, density, network, superstructures and lifecycle in clinical samples of normal tissues, benign tumors, cancers, paracancer tissues, and metastatic cancers.

RESULTS There is no cytocapsular tubes (CTs) in normal organ tissues. Among the 126 benign tumor samples (covering 38 subtypes) from 126 patients, $86.6 \%$ do not have CTs and the other $13.4 \%$ show benign-to-malignancy transitions with CTs. Of the 8,061 solid cancer tissue 
and paracancer samples from 7,125 patients, $100 \%$ of cancers (including carcinomas in situ), 100\% of paracancer tissues, and $100 \%$ of metastatic cancer tissues/organs, harbor large quantities (from thousands to hundreds of thousands) of CTs with many cancer cells in migration inside.

\section{CONCLUSION}

Cytocapsular tubes and networks provide membrane-enclosed physical freeway systems for clinical cancer cell metastasis to neighboring and far-distance tissues and organs.

\section{Introduction}

Cancer is a leading cause of human mortality ${ }^{1}$. It has been estimated that approximately 19.3 million new cancer cases and approximately 10 million cancer deaths occurred worldwide in 2020 alone $^{1}$. Cancer metastasis is the major source of cancer lethality ${ }^{2-6}$. The verifiable identification of cancer metastasis mechanisms is a prerequisite for the effective prognosis, diagnosis, pharmacotherapy and treatment for patients with suspicious, progressive, symptomatic, metastatic and recurrent cancers, for effective drug development, for minimizing side effects and sufferings, and for reliably determining the benefits of treatments. Prognosis, diagnosis and treatment responses are usually assessed with the application of radiographic/ MRI imaging at millimeter $(\mathrm{mm})$ level, which fails to detect the cancer metastasis pathways at micrometer $(\mu \mathrm{m})$ level $^{7-9}$. The methods of precise identification of initial cancerous transformation at $\mu \mathrm{m}$ level in tissues are absent. Therefore, there is an urgent need for verifiable identification of cancer metastasis mechanisms and biomarkers that effectively detect cancer metastasis physical pathways with high sensitivity and specificity. 
Cancer cell metastasis proceeding unprotected through tissues in vivo would encounter numerous heterogeneous neighbor cells and diverse extracellular matrices (ECM), all of which function as both migration clues and substantial environmental obstacles impeding the migration and long distance translocation of cancer cells. Although a number of hypotheses have been proposed ${ }^{10-16}$, consistent models are still missing that could explain the mechanisms underlying cancer metastasis to different sites.

Recently, we discovered that previously unknown membrane-enclosed organelles, dubbed cytocapsular tubes $(\mathrm{CTs})^{17}$, can conduct efficient cell translocation (Fig. S1A; Movies S1-2 ). We developed anti-CM-01 antibodies that recognize a CT membrane protein marker in-vitro (Fig. S1A). We have now used the same membrane protein marker, to investigate CTs in 8,061 solid cancer and paracancer tissues from 7,125 cancer patients (covering 202 kinds of cancers, and including cancers, paracancer and metastatic cancer tissues). We found that the cancer cells in all examined 202 kinds of cancers employ a similar mechanism for near- and long-distance metastases based on CT networks.

\section{Methods}

\section{Patients and Sample Collection}

The sources of clinical samples are described in Fig. 1. The patients providing formalin-fixed paraffin-embedded (FFPE) tissue samples gave informed consent that indicated that they understood that the biopsies (needle biopsy or surgical biopsy) were performed for research purposes only. Comparative deidentified samples of normal tissues, benign tissues, carcinoma in situ, cancer, paracancer, metastatic tissues with their cancer stages identified according to the TNM system (cancer stages: 0, I, II, III, IV) were obtained from archival materials. The cancer, 
paracancer and metastatic cancer tissues, in which the original cancer niches were identified by indicated cancer specific molecular markers, were identified by hospital pathology laboratories and obtained from archival materials.

\section{Histology and Immunohistochemical testing}

Cancer lesion samples were obtained from the index patients from clinical hospitals in USA (8,036 specimens from 7,088 patients, US Biomax; others samples directly from clinical hospitals). Samples were processed for histopathological evaluation with the use of hematoxylin and eosin (H\&E) staining. Immunohistochemical (IHC) fluorescence tests were performed to stain CTs using rabbit anti-CM-01 monoclonal and polyclonal primary antibodies (self-made), mouse anti-gamma-actin monoclonal primary antibodies (Abcam, ab123034) (Fig. S1 ).

\section{Data collection}

Cytocapsular tubes (not sectioned, longitudinally sectioned, and cross sectioned) without degradation (3 6 $6 \mathrm{~m}$ in measured diameter) were counted using a fluorescence microscope and ImageJ. The presence of CTs degrading into thick strands $(1 \sim 2 \mu \mathrm{m}$ in measured diameter), thin strands $(0.2 \sim 1 \mu \mathrm{m}$ in measured diameter), or the disintegration state were reported without quantification.

\section{Statistical analysis}

For each specimen, the number of fully intact cytocapsular tubes was counted in 5 areas $(0.35 \mathrm{~mm} \times 0.35 \mathrm{~mm}$, length $\mathrm{x}$ width) of the sample (top, bottom, left, right, and center), and the

CT density $\left(\mathrm{CT} / \mathrm{mm}^{2}\right)$ was calculated and determined for each area. The average CT density across the 5 sites was treated as the specimen's overall CT density. 


\section{Results}

\section{Cancer metastasis via cytocapsular tubes in 202 kinds of cancers}

Normal tissues: In the 6 types of normal tissues analyzed immunohistochemically (breast, colon, liver, lung, prostate, and stomach; $n=1$ to 3 patients for each type, $n=14$ patients in total), no cytocapsular tubes were found (CT, 0\%) (Fig. 2A and Fig. S2A, 2D, ).

Benign tumors: Of the 6 types (breast, lung, prostate, stomach, liver, colon; covering 38 subtypes) of clinical benign tumor samples ( $n=1$ to 15 patients of each subtype, $n=126$ patients in total), approximately $86.6 \%$ don't present CTs; however, approximately $13.4 \%$ harbor CTs, displaying the transition of a benign tumor to a malignant tumor (Fig. 2A, and Fig. S2B, 2E and Table S1 ). CTs are invisible in conventional hematoxylin \& eosin staining assay or in IHC staining assays with multiple popular cancer protein marker antibodies (such as anti-ER, anti-PR and anti-HER2 antibodies for breast cancer; anti-MSH-2 antibody for colon cancer) (Figs.S2-3 ), but visible in IHC staining assays with anti-CM-01 antibodies in multiple types of cancers, including cancers in breast, colon, oral and thyroid (Figs. S2-S5 ).

Carcinoma in situ: This is the early stage before spreading. In breast carcinoma in situ tissues $(\mathrm{n}=12), 100 \%$ of them have plenty of CTs and the average CT density is high up to $106 \mathrm{CT} / \mathrm{mm}^{2}$ (Excel S1).

202 subtypes of cancer: Next, we performed CT analyses in 202 subtypes of cancers. In the 6 , 837 cancer tissue samples from 6,795 cancer patients (covering 202 subtypes of cancer tissues), all of them (100\%) harbor CTs with various CT densities, and cancer cells were found in migration inside the CTs (Figs.2-3, S2, Tables 1-2, Excel S1-2 ). These 202 subtypes of cancers cover up to 30 kinds of organs: adrenal gland, bladder, bone, brain, breast, cervix uteri, colon, esophagus, gallbladder, head/neck, intestine, kidney, liver, lung, lymph node, oesophagus, oral 
cavity, ovary, pancreas, penis, prostate, rectum, skin, spleen, stomach, testis, thymus, thyroid, uterus, and vulva ( $n=3 \sim 2774$ patients per type of organ). The CT densities in cancers of these 30 kinds of organs vary in a range from 6 to $111 \mathrm{CT} / \mathrm{mm}^{2}$ (Figs.2-3, Excel S1-2 ).

Paracancer: This is a state tissues must pass towards solid cancer metastasis. We performed IHC staining analyses with clinical paracancer tissues (namely "normal adjacent tissue, NAT") of breast and prostate cancers, and found that these paracancer tissues contain plenty of CTs and CT networks with many cancer cells migrating in CTs (Fig.2). Next, we performed IHC staining analyses with paracancer tissues of 12 types of cancers (covering 64 subtypes of cancers) in bladder, breast, esophagus, colon, intestine, kidney, liver, lung, ovary, pancreas, stomach and uterus (Fig. 4). In these paracancer tissues of 64 subtypes of cancers ( $n=3 \sim 365$ samples per subtype), $100 \%$ paracancer tissues harbor plenty of CTs. The CT densities of these paracancer tissues range from 67 to $114 \mathrm{CT} / \mathrm{mm}^{2}$ (Fig. 4). There are many cancer cells migrating in all the CTs in paracancer tissues of all the 64 cancer subtypes (Fig. 4).

Metastatic cancers: Subsequently, we investigated CTs in clinical metastatic mucinous adenocarcinoma from colon in liver, metastatic adenocarcinoma from ovary in epiploon, metastatic breast adenocarcinoma in lymph node, and metastatic mucinous adenocarcinoma from unknown sites in epiploon, $100 \%$ of them harbor numerous CTs with many cancer cells migrating in CTs (Fig. S6 ). We performed CT analyses in 9 types of metastatic cancer tissues in multiple metastatic sites, and found that $100 \%$ of these clinical metastatic cancers harbor a lot of CTs with many cancer cells in migration in CTs (Fig.5A). We performed CT analyses in more metastatic cancers with successive cancer-paracancer-metastatic sites in breast, colon, pancreas and ovary, $100 \%$ of these samples along the cancer-paracancer-metastatic site axis harbor plenty 
of CTs with increased CT density in paracancer tissues comparing to cancer original niches and metastatic cancers (Fig.5B).

\section{Lifecycle of cytocapsular tubes in clinical tissues}

We analyzed the acquired $>60,000$ frames of IHC fluorescence microscope images of the examined 8,061 cancer/paracancer/metastatic samples taken from 7,125 patients covering 202 kinds of cancers, and found variations in CT quantity, density, morphologies, distribution, CT networks, stages in CT life cycle, and superstructure textures, displaying a huge diversity of CTs and networks in all kinds of clinical cancers. Thus, based on the comprehensive analyses of these images, we can identify a lifecycle of cytocapsular tubes in clinical cancers. It can be categorized into 5 successive stages: 1) CT initiation; 2) CT elongation, network formation and cancer cell migration inside; 3) CTs and networks invasion into and pass through paracancer tissues; 4) CTs and CT networks cross tissues and organs and reach neighboring and far-distance destinations; 5) CT degradation and decomposition.

In the initiation phase, individual cancer cells generate cytocapsulas that envelope the cancer cells. Cancer cells migrate inside and generate elongated CTs. The rear part of the CTs display shrunk and thin-tailed morphologies as they are not well anchored in the ECM (Fig.S7A). CTs continue to elongate and form long CTs with consistent diameter/width (2 6 $4 \mathrm{~m}$ in diameter). Individual elongated CTs aggressively invade into neighboring loose and compact tissues, even invade through the tightly-connected and thickness-enhanced epithelial layer of terminal ducts in breast cancer (Fig.S7B). Membrane-enclosed CTs interconnect to each other and form superlarge 3D CT networks and provide membrane-enclosed 3D freeway systems that allow multiple migration directions for CT-conducted cancer cell dissemination in tissues (Fig.S8). 
Elongated CTs generate curved and entangled CT masses that occupy spaces and create CT mass cavities (CMCs). Locally, still-free cancer cells invade into CTs and migrate away via CTs , which creates free (liquid-filled) spaces and cavities that grow in size (Figs.S9A-B). After CT masses degrade and decompose, the empty but liquid filled CT mass cavities (CMCs) are left in the compact cancer tissues (Figs. S9C). Numerous CTs align together and form CT bundles and invade into compact tissues (Fig.S10A). Nearby cancer cells invade into CTs and migrate away, CT masses occupy the spaces while few/no cancer cells remain outside of CTs (Fig. S10B). Cancer cells generate highly curved and coiled CTs, and invade into compact tissues in multiple directions, providing more freeways for cancer cell metastasis (Fig. S11). Free cancer cells invade into CTs generated by other cancer cells, make more CTs and CT networks, and escape to neighboring and far-distance destinations, leaving no free cancer cells beyond CT masses (Figs.S12-13). Cancer cells can generate highly curved CT masses and big curved CT masses, which provide more closed CT freeways for cancer cell invasion into CTs for metastasis (Fig.S14). Cancer cells generate multiple layered, highly curved, coiled superstructures invading into compact tissues, providing large CT contacting surfaces for cancer cell invasion into CT networks for cancer cell metastasis (Fig.S15). During CT conducted cancer metastasis, cancer cells engender many kinds of different complex superstructures for invading into and pass through diverse compact tissues (Fig.S16), huge, dozens-layers complex superstructures invading into tissues with diverse densities (Fig. S17), highly compact cone-shaped superstructure (Fig. S18A), or multiple layered complex superstructures (Fig.S18B). Massive CT clusters can powerfully invade into all kinds of loose and compact tissues (Figs.S19-20). Straight, curved and coiled CTs interconnect together and form large CT networks for cancer metastasis. Even though some parts of CT networks decompose due to CT degradation, the left 
CT networks remain capable of providing CT efficient freeway systems for cancer cell metastasis progression. Thus, CT networks display potent damage resistance capacities and provide efficient CT freeway systems for cancer metastasis (Fig.S21). Subsequent CT degradation employs a multiple-step format: 1) numerous dot membranes on CT surfaces collapse, 2) CTs form a thin CT strand-interconnected network in tube shapes; 3) thin CT strands decompose, disconnect and form individual CT strands; 4) disconnected thin CT strands, decompose and disappear in a complex manner (Figs.S22-24).

The numbers of CTs are always much larger than those of circulating system vessels (blood/lymph vessels) (Fig. S25A). In most instances, plenty of CTs pass through, surround and embed circulating system vessels (blood vessels and lymph vessels) without invading into blood vessels and lymph vessels (Fig. S25A). In the examined 8 kinds of organs, the average number ratio of CTs versus blood and lymph vessels in cancers, paracancer tissues and metastatic cancers is around 1000 (Fig. S25B ). In the late (but not early) stage cancers (stage III or IV), CTs invade into the tightly connected endothelial layers of approximately 1 in $10^{4}$ blood/lymph vessels and release cancer cells into circulation system vessels (Fig.S26A). Approximately $1 \%$ of CT invaded blood/lymph vessels present leak/disassembly followed by local blood cell spreading (Figs.S26B-C). CT analyses with needle biopsy samples provide a linear CT scan in multiplelayer tissues (Fig.S27).

\section{Cytocapsular tube based cancer metastasis grading}

Based on the above observations and data of cancer CT numbers, density, degradation, and metastasis situations of the 8,061 solid cancer and paracancer tissue samples from 7,125 cancer patients (covering 202 kinds of cancers), we suggest a five stage CT-based cancer metastasis 
(CM) grading reference: CM0, CM1, CM2, CM3, and CM4 (Table S3). On the basis of the comprehensive analyses of the cancer stages and CT based CM grade of 7251 benign tumor and cancer patients, we found relationships between cancer stages and CT based CM grades listed in

\section{Table S4.}

\section{Discussion}

It is vital to understand the long-term elusive mechanics underlying cancer metastasis ${ }^{18-25}$. Here, we investigated the metastasis mechanics of 202 kinds of clinical cancers, and evidenced that cancer cells generate substantial membrane-enclosed freeway systems formed by cytocapsular tubes and CT networks that conduct cancer-cell metastases to neighboring and far-distant tissues and organs; this was found to be the case in all 202 kinds of solid cancers tested.

Our data indicate that cancerous cells upon becoming malignant decide to generate extra-cellular cytocapsular tubes initiated by a not-yet-known mechanism. CTs are used by the originator cells and other cancer cells. From there on, cancer cells can migrate in large quantity CTs and protected from outside attacks. CTs can be very long consistent with far-distance metastasis. While most of our data shown here are obtained from snapshots of clinical cancers samples received from cancer tissue banks, the emerging picture is confirmed by clinal data from live cancer patients with cancers in 9 kinds of organs: breast, colon, lung, oral, pancreas, prostate, skin, testis, and thyroid.

It was unforeseen that cytocapsular tubes would be ubiquitously used as physical freeways for cancer metastasis in all 202 kinds of examined solid cancers. Membrane-enclosed homogeneous CTs shield cancer cells from external heterogeneous obstacles of neighbor cells and ECM, and 
significantly facilitate efficient cancer cell dissemination. 3D CT networks provide substantial physical freeways leading in multiple and expanding directions for cancer cell metastases to fardistance tissues and organs. Collective and self-organization activities of long CTs, large networks and superstructures augment collective cancer metastasis through dense, compacted and diverse tissues, cross organs and reach far-distance destinations. The number of cancer cellfilled CTs is approximately 1000-fold larger than that of blood and lymph vessels, and only approximately one 1 of 10,000 blood/lymph vessels present CT-vessel invasion in late stage cancers where cancer cells are released into the circulation systems. This is consistent with clinical observations that there are rare circulating cancer cells (CTCs) in the circulation system $^{26-28}$. These above observations evidenced that the ubiquitous and large quantities of CT networks provide the major physical pathways for neighboring and far-distance cancer metastases in cancer patients.

It is essential to precisely evaluate the cancer metastasis status in prognosis, before and after treatments, and during cancer management to achieve better outcomes for patients. So far, a quantitative and precise cancer metastasis evaluation system is absent ${ }^{2-3,29}$. The cytocapsular tube-based cancer metastasis (CM) grading described here may facilitate the accurate evaluation of cancer cell status and metastasis, thereby improving therapy outcomes. To take advantage of the possibilities for the precise and qualitative evaluation of CT-based cancer metastasis, more clinical analyses are required. In the future, we expect that CT-based drug and technology development will significantly improve cancer pharmacotherapy and management.

Supported by a grant (to Dr. Yi) from Cytocapsula Research Institute Fund for Cytocapsular Tube Conducted Cancer Metastasis Research, and a grant (to Dr. Yi) from Cellmig Biolabs Inc Fund for Cytocapsular Tube Cancer Metastasis Research. 
We greatly acknowledge Dr. Ed Harlow, Harvard Medical School (USA) and Cancer Institute of University of Cambridge (UK), Dr. Jon Aster, Harvard Medical School, Dr. Jelle Wesseling, Leiden University (Netherlands), Dr. David Golan of Harvard Medical School (USA), and Steven Ganshirt, MD of Northwestern University Medical School for their help and meaningful discussion in the study.

\section{Reference:}

1. Sung H, et al, Global cancer statistics 2020: GLOBOCAN estimates of incidence and mortality worldwide for 36 cancers in 185 countries. CA Cancer J Clin 2021; Online ahead of print.

2. Chaffer CL, Weinberg RA. A Perspective on Cancer Cell Metastasis. Science 2011; 331:1559-64

3. Friedl P, Wolf K. Tumour-cell invasion and migration: Diversity and escape mechanisms. Nat Rev Cancer 2003; 3:362-374.

4. Steeg PS. Targeting metastasis. Nat Rev Cancer. 2016 Apr; 16: 201-218.

5. Massagué J, Obenauf AC. Metastatic Colonization. Nature 2016; 529: 298-306

6. Barbolina MV, et al. Microenvironmental regulation of ovarian cancer metastasis. Cancer Treat Res 2009; 149:319-334.

7. Thiery JP, Acloque H, Huang RY, Nieto MA. Epithelial-mesenchymal transitions in development and disease. Cell 2009; 139:871-890.

8. Franz CM, Jones GE, Ridley AJ. Cell migration in development and disease. Dev Cell 2002; 2:153-158.

9. Mehlen P, Puisieux A. Metastasis: a question of life or death. Nat Rev Cancer 2006; 6:449-58

10. Stephen Paget. The distribution of secondary growths in cancer of the breast. The Lancet 1889; 133:571573.

11. Fidler IJ.The pathogenesis of cancer metastasis: the `seed and soil' hypothesis revisited. Nat Rev Cancer 2003; 3:453-8

12. Erler JT, et al. Lysyl oxidase is essential for hypoxia-induced metastasis. Nature 2006; 440:1222-6.

13. Hanahan D, Weinberg RA. Hallmarks of cancer: the next generation. Cell 2011; 144:646-74.

14. Turajlic S, Swanton C. Metastasis as an evolutionary process. Science 2016; 352:169-75.

15. Chiang AC, Massagué J. Molecular basis of metastasis. N Engl J Med 2008;359:2814-23.

16. Valastyan S, Weinberg RA. Tumor metastasis: molecular insights and evolving paradigms. Cell 2011; 147: 275-292.

17. Yi F, Wagner G. Cytocapsular tubes conduct cell translocation. Proc Natl Acad Sci U S A 2018; 115 : E1137-E1146.

18. Sporn, M.B. The war on cancer. Lancet 1996; 347, 1377-1381.

19. Kinzler, K.W., and Vogelstein, B. Landscaping the cancer terrain. Science 1998; 280, 1036-1037.

20. Chambers, A.F., and Matrisian, L.M. Changing views of the role of matrix metalloproteinases in metastasis. J. Natl. Cancer Inst 1997; 89, 1260-1270.

21. Johnson, J.P. Cell adhesion molecules of the immunoglobu- lin supergene family and their role in malignant transformation and progression to metastatic disease. Cancer Metastasis Rev 1991; 10, 11-22.

22. Chiang AC, Massagué J. Molecular basis of metastasis. N Engl J Med 2008; 359:2814-23.

23. Zetter BR. The cellular basis of site-specific tumor metastasis. N Engl J Med 1990; 322:605-12.

24. Roodman GD. Mechanisms of bone metastasis. N Engl J Med 2004; 350:1655-64.

25. Birkbak NJ, McGranahan N. Cancer genome evolutionary trajectories in metastasis. Cancer Cell 2020; $13 ; 37(1): 8-19$.

26. Ashworth TR.. A case of cancer in which cells similar to those in the tumours were seen in the blood after death. Med J Aust. 1869;14:146-7.

27. Nowell PC.. The clonal evolution of tumor cell populations. Science. 1976;194:23-8.

28. Dawson S, et al. Analysis of circulating tumor DNA to monitor metastatic breast cancer. N Engl J Med. 2013;368:1199-209.

29. Boire A, et al. Brain metastasis. Nat Rev Cancer 2020; 20:4-11. 


\section{Figure legends:}

\section{Figure 1. Origen and Enrollment of Clinical Samples from 7,253 Patients.}

In the 7,253 cancer patients, 846 cancer patients provided both cancer and paracancer tissues, 42 cancer patients provided 84 samples (2 tissue samples per patient), and 5,907 cancer patients provided one tissue sample each. In the 24 cancer patients, each provided with 1 original cancer sample, 1 paracancer sample and 1 metastatic cancer sample.

Figure 2. Cytocapsular tubes that are ubiquitously present in clinical solid cancer tissues (but not in normal tissues or benign tumors) are membrane-enclosed, physical freeways of cancer cell metastasis.

A: Comprehensive analyses of 6 kinds of normal human tissues, 38 kinds of benign tumors in 6 kinds of tissues, and 202 types/subtypes of solid cancers in 30 kinds of tissues evidence that CTs, which are ubiquitously distributed in solid cancers but not in normal tissues or non-transformed benign tumors, are physical freeways of cancer cell metastasis and dissemination. B: Representative fluorescence microscope image of a clinical pancreas carcinoma stained with DAPI, anti-CM-01 and anti- $\gamma$-actin antibodies in immunohistochemistry (IHC) assays. A long and highly curved, membrane-enclosed cytocapsular tube (CT) with many hairpins, turns and coils (white arrows, measured length is approximately $5400 \mu \mathrm{m}$ in the sectioned specimen analyzed by ImageJ), forming a super-large structure in an irregular morphology. Outer side that 
well-formed structure, there are many CT strands (CTS) degraded from another layer of superlarge CT structure(s). C: Representative fluorescence microscope image of a clinical breast cancer with many aligned CTs (white arrows) and CT bunches. Breast cancer cells (yellow and orange arrows) migrate in the long and membrane enclosed freeways of CTs. Indicated are: longitudinally sectioned CT fragments with longitudinally sectioned cells migrating in CTs (yellow arrows, in a thin and long morphology shaped by CT membranes), cross sectioned CTs with cross sectioned cells in migration in CTs (orange arrows), and cross sectioned CT surfaces without cells (purple arrows). Scale bar, $10 \mu \mathrm{m}$.

Figure 3. Characterization of cytocapsular tubes in 202 kinds of clinical cancers in 30 types of human organs.

The cancer patient and tissue sample collection underwent randomization. The cytocapsular tube presence, percentages and densities in all the 6,837 examined cancer tissue samples from 6,795 cancer patients (covering 202 kinds of solid cancers and 30 types of organs).

\section{Figure 4. Cytocapsular tubes are massively distributed in paracancer tissues.}

A: Representative fluorescence microscope image of paracancer or "normal adjacent tissues (NAT)" of a breast cancer with massive long, curved and entangled CTs (green color, white arrows) conducting cancer cell metastasis. There are many breast cancer cells in migration (orange arrows, the nuclei are in blue color) in the thousands of thousands of massive CTs. These images show that previously thought "normal adjacent tissues" are not real normal tissues, but harbor thousands or millions of membrane-enclosed highways of CTs conducting massive cancer 
cell dissemination. B: Table of characterization of CTs in the examined clinical paracancer tissues of 64 subtypes of cancers in 12 kinds of organs.

Figure 5. Cytocapsular tubes (CTs) conduct crossing-organ cancer metastasis in solid cancers.

A: Characterization of CTs in crossing-organ cancer metastasis from original cancer niches (in 9 kinds of organs) to multiple far-distance and crossing-organ dissemination. B: Characterization of CTs in 8 kinds of cancers with crossing-organ metastasis from examining CTs in original cancer niches, paracancer tissues, and metastatic cancer tissues.

\section{Supplementary Methods}

Proteome characterization of cancer cell cytocapsulas and cytocapsular tubes and cytocapsular tube protein marker identification.

Multiple stable dual SILAC labeled human cancer cells $\left(100 \%\right.$ SILAC labeling with ${ }^{13} \mathrm{C}_{6},{ }^{15} \mathrm{~N}_{2^{-}}$ Lys and ${ }^{13} \mathrm{C}_{6},{ }^{15} \mathrm{~N}_{4}$-Arg) of pancreas cancer cell Bxpc3, non-small cell lung cancer cell A-427, breast cancer cell MCF-7, and colon cancer cell SK-CO-1 cells were cultured on 3D-Matrigel matrix to grow cytocapsulas and cytocapsular tubes ${ }^{1}$. With cytocapsulae and cytocapsular tube ecellularization and ecellularized cytocapsulae and cytocapsular tube collection methods, SILAC labeled, ecellularized, and cell-free cytocapsulas and cytocapsular tubes were collected, followed 
by SDS-gel purification and LC-MS/MS proteome analyses. We identified 412 cytocapsula and cytocapsular tube proteins. Protein marker candidate screenings were performed in cancer cell cytocapsulae and cytocapsular tubes in vitro with antibodies and IHC-fluorescence microscope analyses. An identified cytocapsular tube membrane protein marker named as CM-01 (Cancer Metastasis-01, an established membrane protein) was used for cytocapsular tube analyses in clinical tissues in vivo.

\section{Supplementary Figure Legends:}

Figure S1. Verification of a cytocapsular tube protein marker in cancer cell cytocapsular membranes in vitro and in vivo.

A: Representative fluorescence microscope images of pancreas cancer cell Bxpc3 cytocapsulae with anti-CM-01 and anti- $\gamma$-actin antibodies in immunohistochemistry (IHC) assays. Bxpc3 cells were implanted onto and cultured on a 3D-Matrigel matrix. Bxpc3 pancreas cancer cells grow cytocapsulas. After ecellulation, Bxpc3 cells existed and left ecellulated cytocapsulas (EC, orange arrows). Cytocapsular membranes (CM, white arrows) of single Bxpc3 cells, Cytocapsular membranes enclosing two or more Bxpc3 cells (CM, purple arrows), and cells outside CMs (cyan arrows) are shown. Panels of DAPI staining, anti-CM-01 antibody staining, anti- $\gamma$-actin antibody staining, and merge are shown. B: Representative image of collective thyroid cancer cytocapsular tubes (CTs) invading into compact thyroid cancer tissues. The bright green color specific for cytocapsular tubes, and the very low green background color in noncytocapsular tube thyroid cancer tissues document the relative enhanced abundance of the protein marker CM-01 recognized by anti-CM-01 antibody and the specific binding ability of 
anti-CM-01 antibodies. Longitudinally-sectioned CTs (white arrows) and cross-sectioned CTs (purple arrows) are shown. Scale bar, $10 \mu \mathrm{m}$.

Figure S2. Cytocapsular tubes are present in clinical cancer tissues, but not in normal tissues or benign tumor tissues.

A-C: Representative images of H\&E stained samples of normal clinical breast tissue, clinical breast benign tumor and clinical malignant breast cancer. D-F: Representative images of IHC fluorescence images of anti-CM-01 (green), anti- $\gamma$-actin (red) and DAPI (blue) stained samples of clinical normal tissue, breast benign tumor and malignant breast cancer, respectively. Conventional H\&E staining method does not clearly show cytocapsular tubes (CTs) in malignant breast cancer tissues, while immunohistochemistry (IHC) staining with anti-CM-01 antibodies shows numerous CTs (white arrows) in malignant breast cancer tissues. Scale bar, $10 \mu \mathrm{m}$.

\section{Figure S3. Anti-CM-01 antibodies, but not conventional popular cancer cell marker antibodies or H\&E staining, show cytocapsular tubes in clinical cancer tissues.}

A: Representative images of H\&E staining (panel 1) and immunohistochemistry (IHC) staining with antibodies recognize ER (estrogen receptor, panel 2), PR (Progesterone receptor, panel 3) and HER2 (human epidermal growth factor receptor 2, panel 4), and anti-CM-01 antibody (panel 5) with clinical breast cancer tissues. Only IHC staining with anti-CM-01 antibody show that there are many cytocapsular tubes (CTs, white arrow, in curved tube-shaped morphologies with 3-6 $\mu \mathrm{m}$ in diameter/width, in red-framed areas) in clinical breast cancer tissues, which are invisible in the panels 1-4. These 5 specimen analyzed here are 5 continuously-sectioned 
specimens $(5 \mu \mathrm{m}$ in thickness, panels $1-5)$ from the same site of the same clinical breast tumor sample. B: Representative images of H\&E staining (panel 1) and IHC staining with antibodies recognizing DNA mismatch repair protein MSH-2 (panel 2), and anti-CM-01 antibody (panel 3) in clinical colon cancer tissues. The specimen in the above analyses are three continuouslysectioned specimens (5 $5 \mathrm{~m}$ in thickness, panels 1-3) from the same site of the same clinical colon cancer sample.

There are many cytocapsular tubes (CT, white arrows) in clinical breast and colon cancer tissues, which are invisible in conventional methods of H\&E staining and IHC staining assay with conventional cancer markers (ER, PR, HER2 for breast cancer, andMSH-2 for colon cancer), but can be clearly detected by anti-CM-01 antibodies in IHC the fluorescence staining assay. There are many colon cancer cells (in cyan color) in migration in CTs in the colon cancer tissue. Scale bar: $10 \mu \mathrm{m}$.

Figure S4. Individual cytocapsular tubes (CTs) potently invade into and pass through compact tissues.

A: Representative image of compact clinical oral cancer tissue. Individual oral cancer cells are propertied with potent capacities to generate long and straight CT freeways crossing condensed and highly compacted tissues in CT conducting cancer cell metastasis. Longitudinal sectioned CT (white arrows) and CT strands (CTS, orange arrows) are shown. B: Representative image of thyroid cancer. Individual thyroid cancer cells exhibit potent abilities to generate CT freeways invading into dense and compacted tissues for cancer cell metastasis. Longitudinal-sectioned or not-sectioned curved CT (white arrows) and cross-sectioned CT (purple arrows) are shown. The

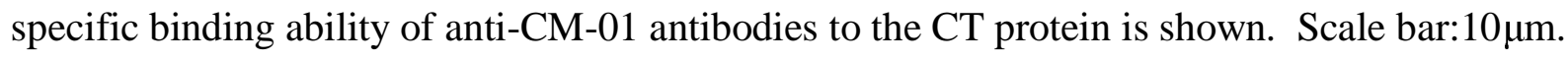


Figure S5. Collective cancer cell cytocapsular tubes (CTs) powerfully invade into compact tissues.

A: Representative image of collective and curved breast cancer CTs invading into compacted tissues. Breast cancer cells present powerful abilities in engendering collective curved CTs for massive cancer metastasis in compacted tissues. Curved, longitudinal-sectioned or not-sectioned CT (white arrows), cross-sectioned CT (purple arrows), and CT strands (CTS, orange arrows) are shown. B: Representative image of cytocapsular tubes (CTs, white arrows) in clinical pancreas cancer tissues. Massive curved CTs invade into compact tissues. Scale bar: $10 \mu \mathrm{m}$.

Figure S6. Long distance cancer metastasis crossing organ/tissue via cytocapsular tubes (CTs) and CT networks.

A: Representative image of metastatic mucinous adenocarcinoma from colon via colon cancer cell CT in liver. Many CTs align and entangle together and form compact CT bundles with multiple sharp tips (top of the panel) invading into the liver with many cancer cells in migration inside colon cancer-cell CTs. Large quantities of liver cells surrounding the colon cancer cell CT cluster are apoptotic and disappear, leaving a big area absent of liver tissues. Longitudinally sectioned colon CTs (white arrows) with longitudinally-sectioned cell in migration in colon CTs (purple arrows), and cross-sectioned colon CTs (orange arrows) in liver are shown. B: Representative image of metastatic adenocarcinoma from ovary via ovary cancer cell CTs in epiploon. There are many straight and curved ovary cancer cell CTs entangled together, invading into compact epiploon tissues, with many ovary cancer cells migrating in CTs. Longitudinally sectioned ovary CTs (white arrows) with longitudinally sectioned cell in migration in CTs 
(purple arrows), and cross-sectioned ovary CTs (orange arrows) in epiploon are shown. C: Representative image of metastatic breast adenocarcinoma from breast via breast cancer cell CTs (and CT networks) in lymph node. Many curved breast cancer cell CTs entangled together and interconnected to form a condensed CT cluster powerfully invading into compact lymph node. Several small CT bunches (cyan arrows) invade into lymph node at the right and left sides. D: Representative image of mucinous adenocarcinoma from unknown site via CT in epiploon. Longitudinally sectioned ovary CTs (white arrows) with longitudinally sectioned cells migrating in CTs (purple arrows) are shown in epiploon. Scale bar:10 $\mu \mathrm{m}$.

\section{Figure S7. Initiation and early elongation of cytocapsular tubes in clinical breast carcinoma}

\section{in situ.}

A: Initiation and early elongation of cytocapsular tube in clinical breast ductal carcinoma in situ. There are plenty of single breast cancer cell cytocapsular tube (CT) in curved morphologies showing shrunk thin tails without CT degradation. The longitudinally sectioned (or not sectioned) CT fragments (white arrows), longitudinal sectioned CT fragments carrying cancer cells (in cyan or blue color and in long and thin morphology) are shown in migration in CTs (cyan arrows), cross sectioned cytocapsula with cell inside (red arrow), and cross sectioned CTs (purple arrows). B: Elongation of CTs in clinical breast ductal carcinoma in situ. Breast cancer cell CTs (white arrows) elongate and aggressively invade into terminal ducts and neighboring tissues. The elongated CTs show consistent diameter/width without the shrunk thin tails. Terminal ducts (TDs) are shown. Scale bar: $10 \mu \mathrm{m}$. 
Figure S8. Cytocapsular tubes (CTs) interconnect and form large 3D CT networks for cancer cell dissemination.

A: Representative image of a part of sectioned network of large and 3D breast cancer cell CT networks with breast cancer cells migrating in CT networks. Cells migrating in CTs can generate new CTs and CT branches in diverse directions. CTs (white arrows) interconnect to each other and form large, 3D and self-organized networks. There are many breast cancer cells (purple arrows) freely migrating in the CT networks, in which most cells are in long and thin morphologies squeezed and shaped by the CT membranes. CT connection node (orange arrows), and longitudinal sectioned CT fragment with longitudinal sectioned cells in CTs (purple arrows) are shown. B: Representative image of a part of sectioned 3D colon cancer cell CT networks with colon cancer cells migrating in CT networks. CT (white arrows), CT connection node (cyan arrows), longitudinal sectioned CT fragment with longitudinal sectioned cells in CTs (purple arrows), cross sectioned CT surfaces with exposed round CT membranes with the tiny CT lumen in a dimmed/darkness in the center (yellow arrows), cross sectioned CT surfaces with shrunk CT membrane and without dimmed lumens (orange arrows) are shown. Multiple ( 3 or more) CTs form a CT hole/grid. In the right part, there is a compact, dense and wide CT network bundle with many long and straight CTs (white brace) align together and interconnect to each other. In a long and straight CT fragment (white brace), there are 5 colon cancer cells (purple arrows) that migrate inside. In the left part, there is a compact and thin CT network bundle with many curved CTs (white thin arrows) entangled together, with many colon cancer cells (purple arrows) in migration in CTs. There is no CT degradation. The grids or holes among CTs in the 3D CT networks are formed during CT interconnection to generate 3D CT networks. Scale bar, 10 $\mu$ m. 


\section{Figure S9. Development of cytocapsular tube (CT) mass cavities.}

A-B: Highly curved/coiled breast cancer cell CTs (white arrows) entangle together and engender CT masses. CT masses occupy spaces in compact breast cancer tissues and form CT mass cavities (CMCs, yellow arrows). Representative image of cross-sectioned CMCs (A) and longitudinally-sectioned CMCs (B, yellow braces) in compact breast cancer tissues are shown. These sectioned CMCs interconnect and form CMC clusters or networks distributed in tissues. Cancer cells surrounding the CT mass cavity can invade into the CTs and leave, and the cavity space size will increase, and the cavities grow into bigger ones. C: Representative image of empty CMCs after CT degradation and decomposition. After CT degradation, CTs disappear, leaving empty CMCs in the compacted cancer tissues. Scale bar:10 $\mu \mathrm{m}$.

Figure S10. Collective cytocapsular tubes (CTs) align together and form CT bundles for massive cancer cell dissemination.

A: Representative image of breast cancer presents many long and straight breast cancer cell CTs aligning together and form compact CT bundles that conduct cancer metastasis to far distance tissues and organs. B: Representative image of breast cancer displays that massive highly curved breast CTs entangled together occupying the space. The cancer cells in that place have invaded into these CTs and relocate to other sites via the CTs, leaving no breast cancer cell here. CT (white arrows), and cell in migration in CTs (purple arrows) are shown. Scale bar:10 $\mu \mathrm{m}$.

Figure S11. Curved cytocapsular tubes (CTs) and CT clusters invade into compact tissues in diverse pathways conducting cancer metastasis in multiple directions. 
A: Representative image of breast cancer tissues. Highly curved and coiled breast cancer cell CTs potently invade into and pass through compacted tissues in multiple and diverse pathways, and conduct cancer metastasis in multiple directions. The cancer cells outside of and close to the CTs can invade into the CTs and relocate to other sites via CTs. B: A representative image presents that numerous highly curved/coiled breast CT masses conquer the place. The cancer cells in the place have invaded into these curved CTs and relocate to other sites via the CTs, leaving no breast cancer cells. Even with the highly curved morphology, these CTs (without degradation) keep the similar and consistent sizes in diameter/width. CT (white arrows), and cell in migration in CTs (purple arrows) are shown. Scale bar: $10 \mu \mathrm{m}$.

\section{Figure S12. Massive breast cancer cell cytocapsular tubes (CTs) conduct cancer metastasis.}

A: Representative image of clinical breast cancer tissues with CT masses. There are many highly curved CTs that pile up and form compact CT masses. The major breast cancer cells in place invade into the CTs and relocate away, leaving very few cells in the center. B: Representative image of breast cancer tissues with CT masses. A large quantity of curved CTs pile up and form large CT masses occupying the spaces. The breast cancer cells in this place invade into these CTs and relocate away leaving no cancer cells outside of CTs. At the top right corner, there are two big cavities caused by the CT degradation and decomposition. CT (white arrows), and cell in migration in CTs (purple arrows) are shown. Scale bar:10 $\mu \mathrm{m}$.

Figure S13. Massive breast cancer cell CTs conduct massive cancer metastasis. There are plenty of hairpin-shaped and highly curved CTs (white arrows) in clinical breast carcinoma with 
cancer cells (purple arrows) migrating in CTs. Massive cancer cells invaded the CTs leaving no cancer cell outside. Some CTs degrade into CT strands (CTS, orange arrows). Scale bar, 10 $\mu$ m.

\section{Figure S14. Highly curved pancreas and lung cancer cell CTs induce cancer metastasis.}

A: Representative image of pancreas cancer with plenty of highly curved pancreas cancer cell CTs randomly distributed in clinical pancreas cancer tissues. At the top, there is a long and highly curved pancreas cancer cell CT with many condensed hairpin-shaped turns and a continuously maintained diameter of $4 \mu \mathrm{m}$. B: Representative image of lung cancer with multilayered, highly curved lung cancer cell CTs. At the bottom, there are multiple layers of CT individuals with regular and irregular turns and curves. The highly curved CT repeats significantly increase the area for contacting more cancer cells, providing more close freeways for cancer cell metastasis in an efficient format. Scale bar, $10 \mu \mathrm{m}$.

Figure S15. Cross-section view of highly curved, coiled, multi-layered pancreatic-cancer

\section{CT super-structures.}

The highly curved and coiled pancreatic cancer cell CTs form multi-layered CT superstructures in pancreas cancer, displaying the powerful capacities of CTs in generating complex superstructures when invading into tissues during the CT conducted cancer metastasis. CT (white arrows), and CT strands (CTS, orange arrows) are shown. Scale bar:10 $\mu \mathrm{m}$.

Figure S16. Cross-section view of two big, round, multi-layered, multi-staged, complex colon cancer CT superstructures.

A representative cross-section view of two big and complex CT super-structures of round morphology. The bigger one displays multiple layers: outer side thin layer, in severe degradation 
into thin CT strands; middle thick layer, many highly curved CTs distributed in a ring circulating the center; and inner side layer, CTs with severe degradation into cloud-like status. At the bottom left corner, most CTs degrade into CT strands (CTS) in diverse thickness in this big complex CT super-structure. These two complex CT super-structures of round morphology and exhibiting multiple layers of multi-staged CTs display the vigorous abilities of collective CTs in engendering diverse and effective superstructures for efficient invasion during conducting cancer metastasis. CT (white arrows), and CT strands (CTS, orange arrows) are shown. Scale bar:10 $\mu \mathrm{m}$. Figure S17. Cross-section view of huge, flattened and oval shaped, multi-layered, complex prostate cancer $\mathrm{CT}$ superstructures.

A: Representative image of cross-section view of a part of a huge, flattened and oval shaped, multi-layered, complex prostate CT superstructure. There are many circulated CTs that form a complex CT superstructure. The purple arrow labeled CT bundle is formed by aligned 4 long and curved CTs with a big hair-pin shaped curve. This huge, multi-layered, complex CT superstructure presents the potent capacities of collective CT invasion in cancer metastasis. Multiple CTs tightly align together and form a CT bundle with enhanced invasion capabilities during conducting cancer cell metastasis in tissues. B: Representative image of a part of crosssection view of a huge, multi-layered, irregular-shaped morphology, multi-CT stages, and complex pancreas cancer cell CT superstructures. In the middle, there is a part of a single, long, highly curved pancreas cancer cell CT with many hairpins, turns, coils and irregular curves. In the outside, there are multiple layered CTs degrade into strands in various sizes in width. CT (white arrows), and CT strands (CTS, orange arrows) are shown. Scale bar:10 $\mu \mathrm{m}$. 
Figure S18. Longitudinal section view of long and big breast cancer CT superstructures in conducting cancer metastasis in compact tissues.

A: Longitudinal section view of a long, big and needle-shaped breast cancer CT superstructure during cancer metastasis. This image shows that many long, curved or straight breast CTs entangle together, and form a long, big, self-organized and needle-shaped CT superstructure, massively and powerfully spur into the highly condensed and compacted tissues, conducting cancer metastasis. There are multiple CTs (orange arrows) extend into the compacted tissue surrounding the CT super-structure including the front. B: Longitudinal view of a long, big, sandwiched, irregular CT superstructure in conducting cancer metastasis. Most CTs are degraded into CT strands (CTS, orange arrows). CT (white arrows), and cells in migration in CTs (purple arrows) are shown. Scale bar: $10 \mu \mathrm{m}$.

Figure S19. Massive invasion of breast cancer CTs into diverse types of tissues during cancer metastasis.

A: A representative image of breast cancer tissue shows that a large quantity of breast cancer CTs massively invade into the neighboring tissue among terminal ducts (TD), and some CTs (purple arrows) even crossed through the tight contacted epithelial cell layers of TD and invade into the TD lumens. B: A representative image presents that massive CTs in three different CT lifecycle stages invade into diverse tissues during breast cancer metastasis. The right CT mass in a big round morphology is in a late CT stage with CT degradation into cloud-like status. In the middle CT mass, most CTs are not in degradation, and some CTs cross the tight TD epithelial layer and invade into the TD lumens (purple arrow). In the bottom CT mass, most CTs degrade 
into thick and thin strands. Longitudinal sectioned CT (white arrows), cross-sectioned CTs (yellow arrows), and CT strands (CTS, orange arrows) are shown. Scale bar:10 $\mu \mathrm{m}$.

\section{Figure S20. Collective CT clusters invade through compact tissues conducting long distance, CT-directed cancer metastasis.}

A. Representative fluorescence microscope image shows that many curved and entangled prostate cancer-cell CTs formed a curved cone-shaped CT network cluster that invades through a compact tissue layer during cancer metastasis. B. Representative image displays numerous curved, coiled and entangled breast cancer cell CT (white arrows) formed collective CT masses that invade into compact tissues in multiple directions, wrapping compact tissues and separating them into several islands. These images document that collective CT clusters can aggressively invade through compact tissues and reach far distance tissues/organs, conducting cancer

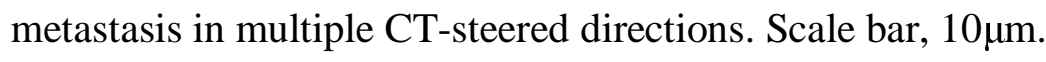

Figure S21. Cytocapsular tube networks possess robust capacities to direct cancer cell metastasis-pathway and are network damage resistant.

A: Representative image of compacted straight breast cancer CT network bunches with CT degradation. There are several big holes in the compact CT network bundle caused by CT degradation and partly CT network decomposition, but there are still some CTs that connect between the small bundles, which enables the 3D CT network bundles to keep interconnections, thus allowing cancer cell metastasis among the whole CT networks to continue and provide CT network damage resistance. B: Representative image of compacted curved CT network masses with severe CT degradation. In the compact and condensed breast cancer CT network masses formed by highly curved, coiled and entangled CTs, part of CTs degrade and networks 
decompose, leaving many big holes in the CT network masses. The remaining 3D CT networks keep interconnections via curved or coiled CTs, which maintains the powerful cancer cell metastasis pathway capacities with CT network damage resistance. Scale bar: $10 \mu \mathrm{m}$.

Figure S22. Cytocapsular tube degradation stage I: point array-membrane collapse and decomposition, and CT strand network-tube formation.

A-B: Representative breast cancer CT degradation from a breast cancer specimen with fresh breast cancer tissues and instant FFPE (formalin-fixed, paraffin-embedded) treatment. There are many tiny points (point array, $<0.1 \mu \mathrm{m}$ in width in measured sizes) in the CT membranes collapse and decompose, leaving plenty of tiny holes $(0.05-0.2 \mu \mathrm{m}$ in width in measured sizes) in CTs. The remaining CT strands interconnect and form CT strand-network in the CT tubes: the whole CTs transiently remain the outline of the original CT, while the CT surfaces are composed of thin or very thin CT strand networks with many point-shaped tiny holes (point array) inside. Subsequently, the membrane degradation continues, connected CT strand networks in the CT tubes decompose into disconnected thin strands until they completely disappear. The white-line framed area is enlarged and shown in panel B. This enlargement shows not-yet-degraded CTs (white arrows), micro CT strand network tubes (purple arrow), and tiny point CT membrane degradation-generated holes (orange arrows). Scale bar:10 $\mu \mathrm{m}$.

Figure S23: Cytocapsular tube degradation stage II: Disconnected CT strands, thin CT strands.

A: Representative image of breast cancer showing degradation of micro CT strand network tubes into CT strands, thin CT strands, and cloud like status. Labeled are: CTs without degradation 
(white arrows), CT strands (orange arrows), thin strands (yellow arrows), and cloud-like status

(purple arrows). B: Representative image of breast cancer cell CT degradation into CT strands and thin CT strands. Linear colon cancer cell CT strands (orange arrows) and thin strands (yellow arrows) sporadically distributed in colon tissues are shown. Scale bar:10 $\mu \mathrm{m}$.

\section{Figure S24. Massive cytocapsular tube degradation.}

A: After cancer cells invade into CTs and migrate away, massive CTs degrade into massive thick and thin strands. There are countless CT strands. B: CT strands continue to degrade into massive very thin strands, followed by decomposition and disappearance. After CT strands disappear, these sites will leave cavities filled with intercellular fluids. Scale bar: $10 \mu \mathrm{m}$.

\section{Figure S25. Cytocapsular tubes and networks dominate cancer metastasis pathways.}

A: Representative fluorescence microscope image of prostate cancer tissue specimen shows that there are several hundreds of CTs (white arrows) and only one micro blood vessel (MBV) in this imaged area. Some CTs degrade into CT strands (CTS, orange arrows). B: Comprehensive analyses of ratios of numbers of CTs and circulatory system vessels (blood vessels and lymph vessels) examined in cancer, paracancer and metastatic cancer for 8 types of cancers in breast, colon, lung, liver, ovary, pancreas, prostate and stomach. Scale bar, $10 \mu \mathrm{m}$.

\section{Figure S26. Cytocapsular tubes invade into micro blood vessels and release cancer cells into} circulation systems at late cancer stages.

A. Representative image of rare CT invasion into micro blood vessels (MBV) in stage III prostate cancer. Approximately 1 in $10^{4} \mathrm{CTs}$ (white arrows) wrap around micro blood vessels, 
aggressively invade into and pass through the tightly connected endothelial layer of micro blood vessel. The cyan arrow labels a CT that protrudes into the micro blood vessel lumen and cancer cells migrate inside. This indicates that cancer cells can be released from the CTs that invade into blood and lymph vessels and subsequently allowing cancer cells release into the circulation systems as circulating tumor cells. There are numerous CTs (white arrow), degraded CT strands (CTS, orange arrows) outside the circulation system vessels. Red blood cells (RBC, purple arrows) are also shown. B. Representative image of a micro blood vessel that is leaky due to CT invasion in stage IV lung cancer. The micro blood vessel with leak caused by CT invasion is full of red blood cells (RBC, purple arrows), which spread and distribute around the local neighboring tissues. C. Representative image of blood vessels with leaks caused by severe CT invasion. This triggered blood vessel disassembly and decomposition in stage IV prostate cancer. There are many CT strands and fragments (CTS, orange arrows) and red blood cells (RBC, purple arrows). The local prostate cancer cells invaded into CTs (white arrows) and migrate away, leaving spaces without tissue cells. Scale bar:10 $\mu \mathrm{m}$.

\section{Figure S27. Continuous multi-layered tissue scanning of cancer cell cytocapsular tube in needle biopsy for cancer prognosis and diagnosis.}

Representative images of multiple sites in needle biopsy with suspicious breast cancer tissues by CT analyses for breast cancer diagnosis. In site 3, there are many breast cancer cell CTs without degradation. In sites 4 and 5, there are numerous breast cancer cell CT strands in degradation. Scale bar: $10 \mu \mathrm{m}$. 
Movie S1: Movie of cancer stem cell (CSC) cytocapsular-tube initiation, generation, and bidirectionally migration in the CSC CT it generated.

A single breast cancer stem cell from the HMLER(CD $\left.44^{\text {high }} / \mathrm{CD} 24^{\text {low }}\right)^{\text {FA }}$ subpopulation ${ }^{1}$ generates a short cytocapsular tube in the 3D Matrigel matrix. Subsequently, it migrates bidirectionally in the CT it engendered. Initially, the cell generates a cytocapsula outside of and disconnected from the plasma membrane. Then the cell moves to the left to elongate the cytocapsular membrane to engender a short cytocapsular tube (CT) in its wake. Then, the cell migrates in the CT to the right. There are several transient spike-like features of still unknown structure dynamically connecting the rear of the cell with the CT membranes. Scale bar:10 $\mu \mathrm{m}$.

\section{Movie S2: Multiple cancer stem cells bi-directionally migrate in a single long CSC cytocapsular tube.}

There are 4 breast cancer stem cell HMLER(CD $\left.44^{\text {high }} / \mathrm{CD} 24^{\text {low }}\right)^{\mathrm{FA}}$ subpopulation cells migrate in a long cytocapsular tube (CT) generated by another breast CSC. The top CSC bi-directionally migrates inside the breast CSC CT. Scale bar: $10 \mu \mathrm{m}$.

\section{References:}

1. Yi F, Wagner G. Cytocapsular tubes conduct cell translocation. Proc Natl Acad Sci U S A 2018; 115 : E1137-E1146. 
Fig. 1

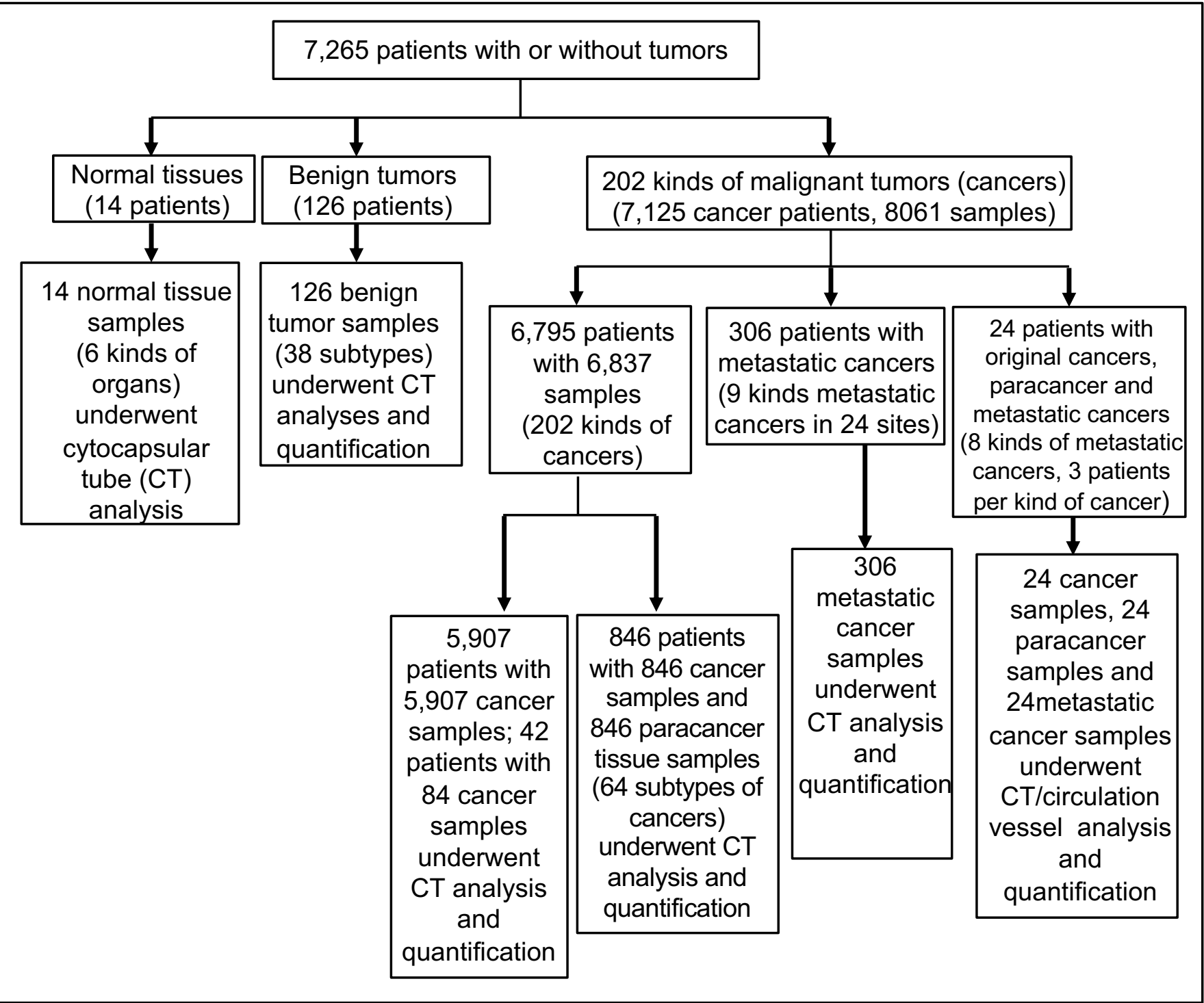




\section{Fig. 2}

was not certified by peer review) is the author/funder. All rights reserved. No reuse allowed without permission.

\begin{tabular}{|c|c|c|c|c|c|c|c|}
\hline \multirow{4}{*}{ Normal tissue } & \multirow{4}{*}{6} & Breast & & 3 & 3 & 0 & 0 \\
\hline & & Liver & & 2 & 2 & 0 & 0 \\
\hline & & Lung & & 3 & 3 & 0 & 0 \\
\hline & & Prostate & & 1 & 1 & 0 & 0 \\
\hline Benign tumor tissue (details in Table S1) & 6 & & 38 & 126 & 126 & 17 & $13.4 \%$ \\
\hline Cancer/malignant tumor (details in Table 2) & 30 & & 202 & 6795 & 6837 & 6837 & $100 \%$ \\
\hline
\end{tabular}

B

\section{Clinical pancreas carcinoma}

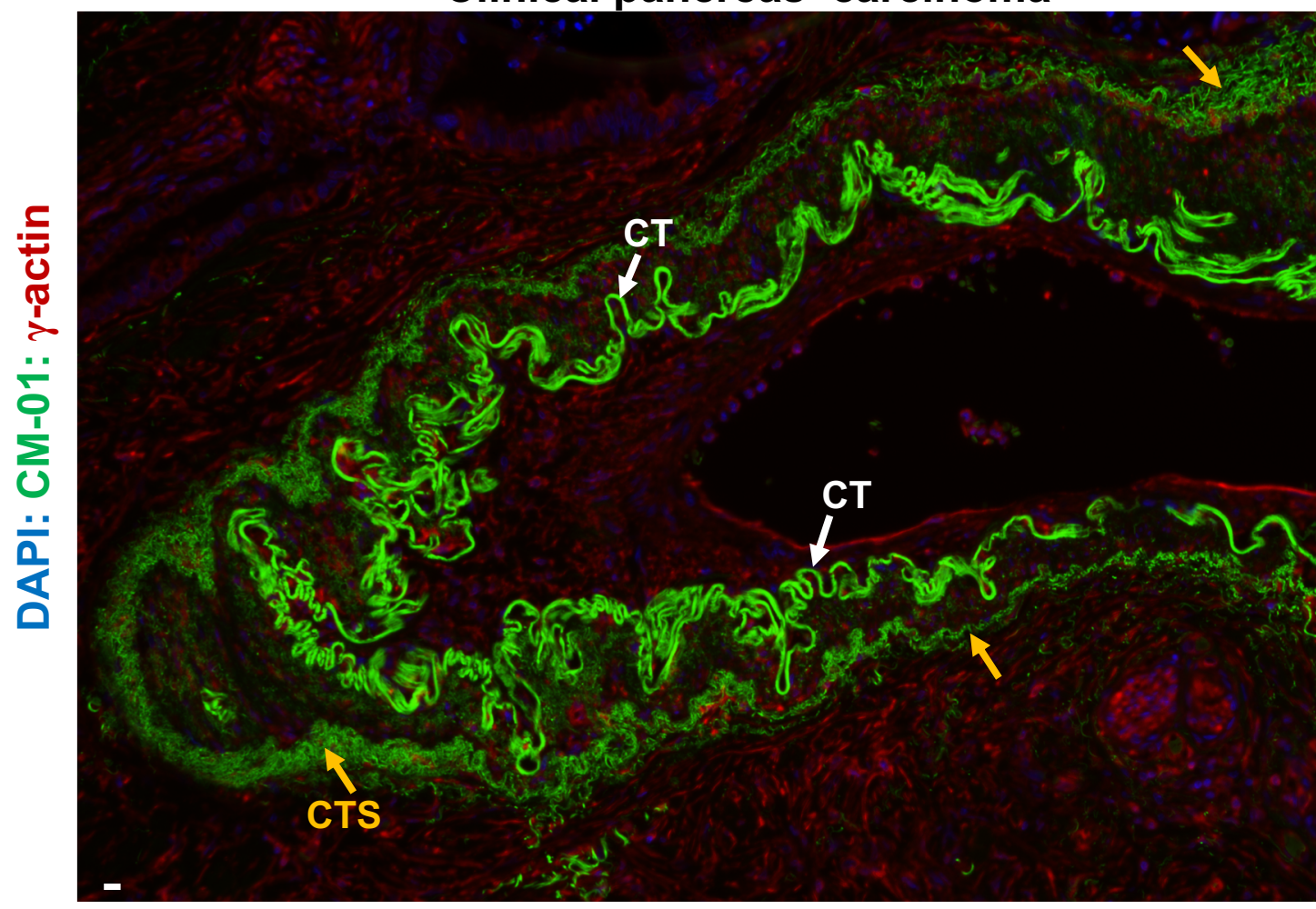

C

\section{Clinical breast cancer}

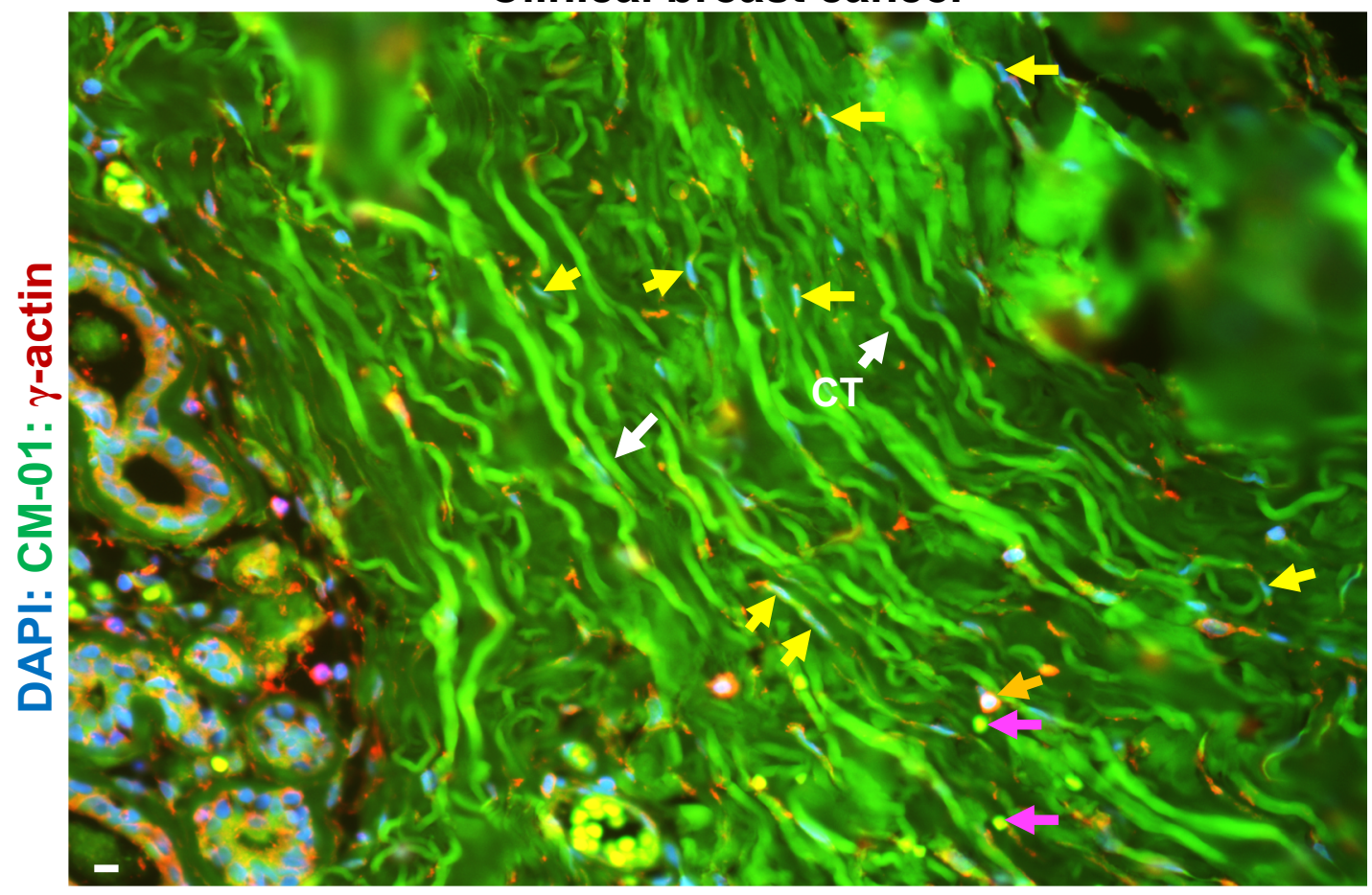




\begin{tabular}{|c|c|c|c|c|c|c|c|c|c|c|c|}
\hline & $\begin{array}{c}\text { Cancer types by } \\
\text { organs, tissues }\end{array}$ & $\begin{array}{c}\text { Cancer } \\
\text { subtypes }^{\#} \\
\end{array}$ & $\begin{array}{c}\text { Tissue } \\
\text { specimen } \\
\text { number } \\
\end{array}$ & $\begin{array}{l}\text { Patient } \\
\text { number }\end{array}$ & Male & Female & $\begin{array}{c}\text { Age } \\
\text { range }\end{array}$ & $\begin{array}{l}\text { Cancer } \\
\text { stages }\end{array}$ & $\begin{array}{c}\text { Tissues with } \\
\text { cytocapsular tubes } \\
\text { (CTs) }\end{array}$ & $\begin{array}{c}\% \text { of cancer } \\
\text { tissues with CT }\end{array}$ & $\begin{array}{c}\text { CT density } \\
\left(\mathrm{CT} / \mathrm{mm}^{2}\right) \\
\text { range }\end{array}$ \\
\hline 1 & Adrenal Gland & 3 & 23 & 22 & 10 & 12 & $26-71$ & I-IV & + & 100 & $6-73$ \\
\hline 2 & Bladder & 5 & 46 & 46 & 30 & 16 & $36-74$ & I-IV & + & 100 & $20-102$ \\
\hline 3 & Bone & 12 & 212 & 210 & 115 & 95 & $21-78$ & I-IV & + & 100 & $7-78$ \\
\hline 4 & Brain & 3 & 124 & 122 & 86 & 36 & $24-67$ & I-IV & + & 100 & $9-109$ \\
\hline 5 & Breast ${ }^{*}$ & 27 & 2786 & 2774 & & 2774 & $18-86$ & I-IV & + & 100 & $6-113$ \\
\hline 6 & Cervix uteri & 7 & 53 & 51 & & 51 & $37-55$ & I-IV & + & 100 & $8-97$ \\
\hline 7 & Colon $^{* *}$ & 6 & 586 & 586 & 323 & 263 & $16-90$ & I-IV & + & 100 & $18-113$ \\
\hline 8 & Esophagus & 5 & 63 & 62 & 41 & 21 & $24-65$ & I-IV & + & 100 & $10-103$ \\
\hline 9 & Gallbladder & 3 & 26 & 26 & 20 & 6 & $37-62$ & I-IV & + & 100 & $8-106$ \\
\hline 10 & Head/neck & 5 & 12 & 12 & 4 & 8 & $41-66$ & I-IV & + & 100 & $12-111$ \\
\hline 11 & Intestine & 3 & 15 & 15 & 10 & 5 & $36-68$ & I-IV & + & 100 & $12-106$ \\
\hline 12 & Kidney & 10 & 123 & 120 & 81 & 39 & $33-65$ & I-IV & + & 100 & $5-98$ \\
\hline 13 & Liver & 6 & 362 & 358 & 231 & 127 & $36-76$ & I-IV & + & 100 & $23-106$ \\
\hline 14 & Lung & 23 & 683 & 676 & 437 & 239 & $27-78$ & I-IV & + & 100 & $45-103$ \\
\hline 15 & Lymph node & 9 & 56 & 56 & 31 & 25 & $21-73$ & I-IV & + & 100 & $26-107$ \\
\hline 16 & Oesophagus & 2 & 4 & 4 & 3 & 1 & $34-54$ & I-IV & + & 100 & $25-98$ \\
\hline 17 & Oral cavity & 5 & 17 & 17 & 10 & 7 & $28-69$ & I-IV & + & 100 & $15-87$ \\
\hline 18 & Ovary & 7 & 83 & 83 & & 83 & $32-61$ & I-IV & + & 100 & $18-107$ \\
\hline 19 & Pancreas & 13 & 222 & 218 & 162 & 56 & $28-74$ & I-IV & + & 100 & $29-111$ \\
\hline 20 & Penis & 2 & 3 & 3 & 3 & & $45-62$ & I-IV & + & 100 & $25-104$ \\
\hline 21 & Prostate & 5 & 875 & 871 & 871 & & $35-77$ & I-IV & + & 100 & $35-110$ \\
\hline 22 & Rectum & 5 & 5 & 5 & 4 & 1 & $32-57$ & I-IV & + & 100 & $45-97$ \\
\hline 23 & Skin & 7 & 122 & 122 & 35 & 87 & $21-79$ & I-IV & + & 100 & $27-108$ \\
\hline 24 & Spleen & 2 & 6 & 6 & 1 & 5 & $36-48$ & I-IV & + & 100 & $32-97$ \\
\hline 25 & Stomach & 6 & 245 & 245 & 123 & 122 & $36-72$ & I-IV & + & 100 & $38-109$ \\
\hline 26 & Testis & 5 & 24 & 24 & 24 & & $41-67$ & I-IV & + & 100 & $37-111$ \\
\hline 27 & Thymus & 4 & 5 & 5 & 4 & 1 & $37-56$ & I-IV & + & 100 & $35-107$ \\
\hline 28 & Thyroid & 5 & 17 & 17 & 10 & 7 & $29-61$ & I-IV & + & 100 & $27-102$ \\
\hline 29 & Uterus & 5 & 26 & 26 & & 26 & $35-56$ & I-IV & + & 100 & $36-108$ \\
\hline \multirow[t]{2}{*}{30} & Vulva & 3 & 13 & 13 & & 13 & $32-55$ & I-IV & + & 100 & 34-108 \\
\hline & Total & 202 & 6837 & 6795 & 2669 & 4126 & & & + & 100 & \\
\hline
\end{tabular}

(\#: Details of 10 or more subtypes are shown in Table S2;* Details of a part of the samples are shown in Excel S1; ** Details are shown in Excel S2 ). 
Fig.4

Clinical paracancer tissues of breast cancer

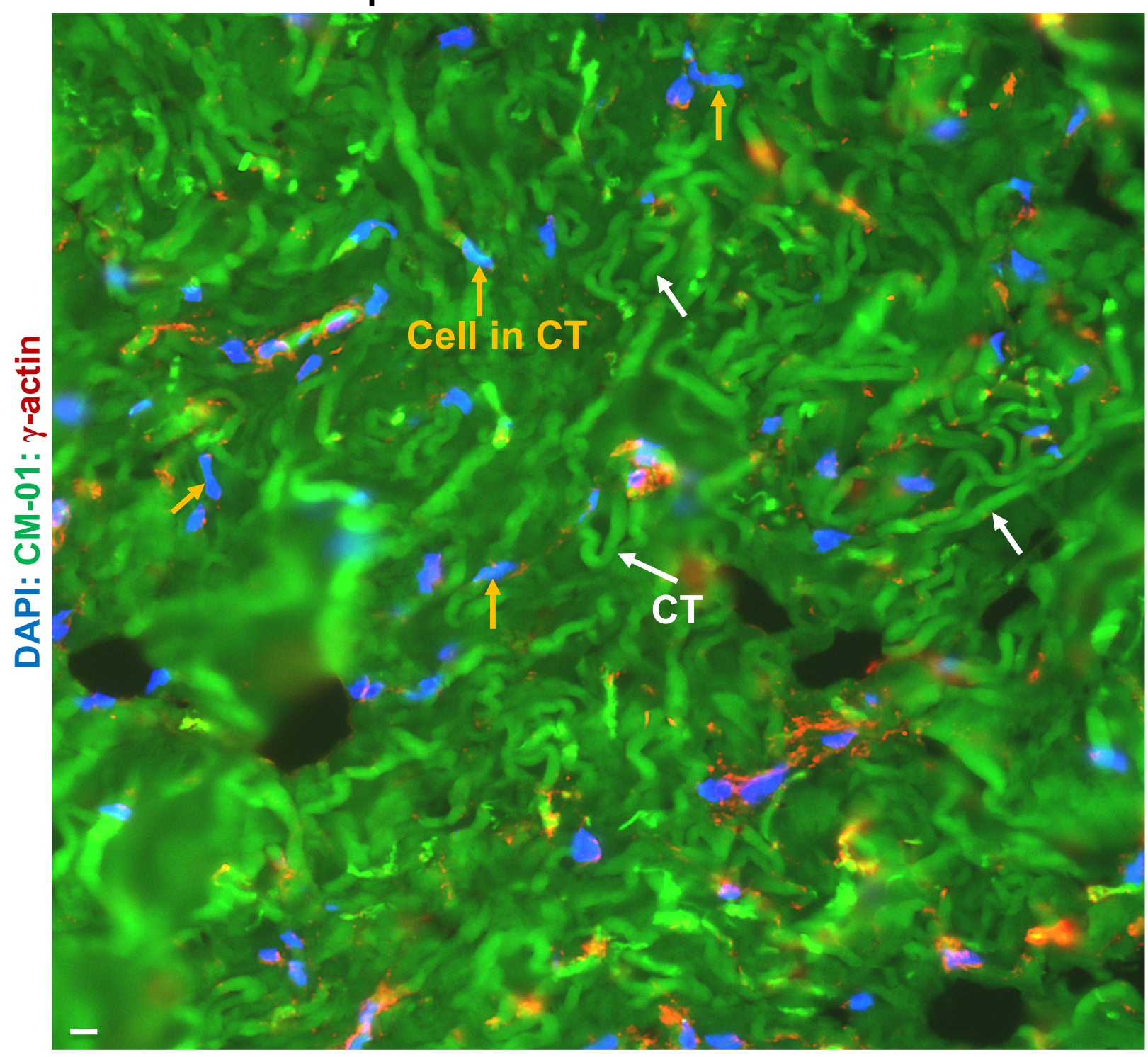

B

\begin{tabular}{|c|c|c|c|c|c|}
\hline Cancer types & Cancer subtypes & $\begin{array}{l}\text { Paracancer tissue } \\
\text { specimen number }\end{array}$ & $\begin{array}{c}\text { Number of paracancer } \\
\text { tissues with } \mathrm{CT}\end{array}$ & $\begin{array}{l}\% \text { of paracancer } \\
\text { tissue with CT }\end{array}$ & CT density \\
\hline Bladder & 2 & 10 & 10 & 100 & 72-101 \\
\hline Breast & 25 & 356 & 356 & 100 & $78-114$ \\
\hline Esophagus & 1 & 5 & 5 & 100 & $72-103$ \\
\hline Colon & 7 & 121 & 121 & 100 & $71-109$ \\
\hline Intestine & 1 & 3 & 3 & 100 & $78-107$ \\
\hline Kidney & 2 & 6 & 6 & 100 & $75-112$ \\
\hline Liver & 6 & 89 & 89 & 100 & $67-103$ \\
\hline Lung & 5 & 65 & 65 & 100 & $82-112$ \\
\hline Ovary & 2 & 12 & 12 & 100 & $79-114$ \\
\hline Pancreas & 6 & 96 & 96 & 100 & $81-107$ \\
\hline Stomach & 6 & 78 & 78 & 100 & $79-113$ \\
\hline Uterus & 1 & 5 & 5 & 100 & $81-102$ \\
\hline Total & 64 & 846 & 846 & 100 & \\
\hline
\end{tabular}




\begin{tabular}{|c|c|c|c|c|c|c|}
\hline $\begin{array}{l}\text { Original cancer } \\
\text { niche }\end{array}$ & $\begin{array}{c}\text { Cancer metastasis } \\
\text { to other organs }\end{array}$ & $\begin{array}{l}\text { Patient } \\
\text { number }\end{array}$ & $\begin{array}{l}\text { Metastatic cancer } \\
\text { tissue number }\end{array}$ & $\begin{array}{l}\text { Number of metastatic } \\
\text { cancer tissues with } \mathrm{CT}\end{array}$ & $\begin{array}{l}\% \text { of metastatic } \\
\text { cancer with CT }\end{array}$ & $\begin{array}{l}\text { CT density } \\
\left(\mathrm{CT} / \mathrm{mm}^{2}\right) \\
\end{array}$ \\
\hline \multirow{5}{*}{ Breast } & lymph node & 45 & 45 & 45 & 100 & $36-104$ \\
\hline & lung & 4 & 4 & 4 & 100 & $65-112$ \\
\hline & liver & 3 & 3 & 3 & 100 & $53-108$ \\
\hline & bone & 3 & 3 & 3 & 100 & $47-102$ \\
\hline & Neck/head & 5 & 5 & 5 & 100 & $68-113$ \\
\hline \multirow{5}{*}{ Colon } & lymph node & 32 & 32 & 32 & 100 & $42-105$ \\
\hline & liver & 4 & 4 & 4 & 100 & $50-102$ \\
\hline & epiploon & 3 & 3 & 3 & 100 & $53-104$ \\
\hline & stomach & 4 & 4 & 4 & 100 & $82-113$ \\
\hline & ovary & 4 & 4 & 4 & 100 & $46-108$ \\
\hline Esophagus & lymph node & 23 & 23 & 23 & 100 & $37-98$ \\
\hline \multirow[t]{2}{*}{ Liver } & lymph node & 34 & 34 & 34 & 100 & $45-107$ \\
\hline & spleen & 5 & 5 & 5 & 100 & 51-109 \\
\hline \multirow{2}{*}{ Lung } & lymph node & 21 & 21 & 21 & 100 & $65-98$ \\
\hline & abdominal cavity & 3 & 3 & 3 & 100 & $62-101$ \\
\hline \multirow{3}{*}{ Stomach } & lymph node & 25 & 25 & 25 & 100 & $52-106$ \\
\hline & liver & 8 & 8 & 8 & 100 & $56-99$ \\
\hline & lung & 7 & 7 & 7 & 100 & $47-104$ \\
\hline \multirow[t]{2}{*}{ Pancreas } & lymph node & 32 & 32 & 32 & 100 & $53-106$ \\
\hline & mesentery & 4 & 4 & 4 & 100 & $34-103$ \\
\hline \multirow[t]{2}{*}{ Prostate } & lymph node & 13 & 13 & 13 & 100 & $47-98$ \\
\hline & stomach & 4 & 4 & 4 & 100 & $54-105$ \\
\hline \multirow[t]{2}{*}{ Ovary } & lymph node & 21 & 21 & 21 & 100 & $44-101$ \\
\hline & liver & 4 & 4 & 4 & 100 & $56-109$ \\
\hline & Tot & 306 & 306 & 306 & 100 & \\
\hline
\end{tabular}

B

\begin{tabular}{|c|c|c|c|c|c|c|c|c|c|}
\hline $\begin{array}{c}\text { Original } \\
\text { cancer } \\
\text { niche }\end{array}$ & $\begin{array}{l}\text { Patient/ } \\
\text { tissue } \\
\text { number }\end{array}$ & $\begin{array}{l}\text { CT density } \\
\text { in cancer } \\
\text { tissues }\end{array}$ & $\begin{array}{l}\text { Paracancer } \\
\text { tissue number }\end{array}$ & $\begin{array}{c}\% \text { of } \\
\text { paracancer } \\
\text { with CT } \\
\end{array}$ & $\begin{array}{l}\text { CT density } \\
\text { in } \\
\text { paracancer }\end{array}$ & $\begin{array}{c}\text { Cancer } \\
\text { metastasis to } \\
\text { organs }\end{array}$ & $\begin{array}{c}\text { Metastatic cancer } \\
\text { tissue (MCT) } \\
\text { number }\end{array}$ & $\begin{array}{c}\% \text { of metastatic } \\
\text { cancer tissue } \\
\text { with CT }\end{array}$ & $\begin{array}{c}\text { CT } \\
\text { density } \\
\text { of MCT }\end{array}$ \\
\hline Breast & 3 & $35-101$ & 3 & 100 & $81-112$ & head/neck & 3 & 100 & $69-103$ \\
\hline Colon & 3 & $76-102$ & 3 & 100 & $92-112$ & Stomach & 3 & 100 & $96-113$ \\
\hline Lung & 3 & $69-110$ & 3 & 100 & $94-113$ & Lymph & 3 & 100 & $89-108$ \\
\hline Liver & 3 & $43-98$ & 3 & 100 & $89-108$ & Spleen & 3 & 100 & $85-107$ \\
\hline Ovary & 3 & $46-92$ & 3 & 100 & 85-109 & Liver & 3 & 100 & $84-105$ \\
\hline Pancreas & 3 & $38-98$ & 3 & 100 & $87-113$ & Stomach & 3 & 100 & $61-104$ \\
\hline Prostate & 3 & $46-103$ & 3 & 100 & $86-108$ & Liver & 3 & 100 & $78-102$ \\
\hline Stomach & 3 & $52-106$ & 3 & 100 & 89-113 & Lung & 3 & 100 & 81-105 \\
\hline
\end{tabular}




\section{Fig.S1}

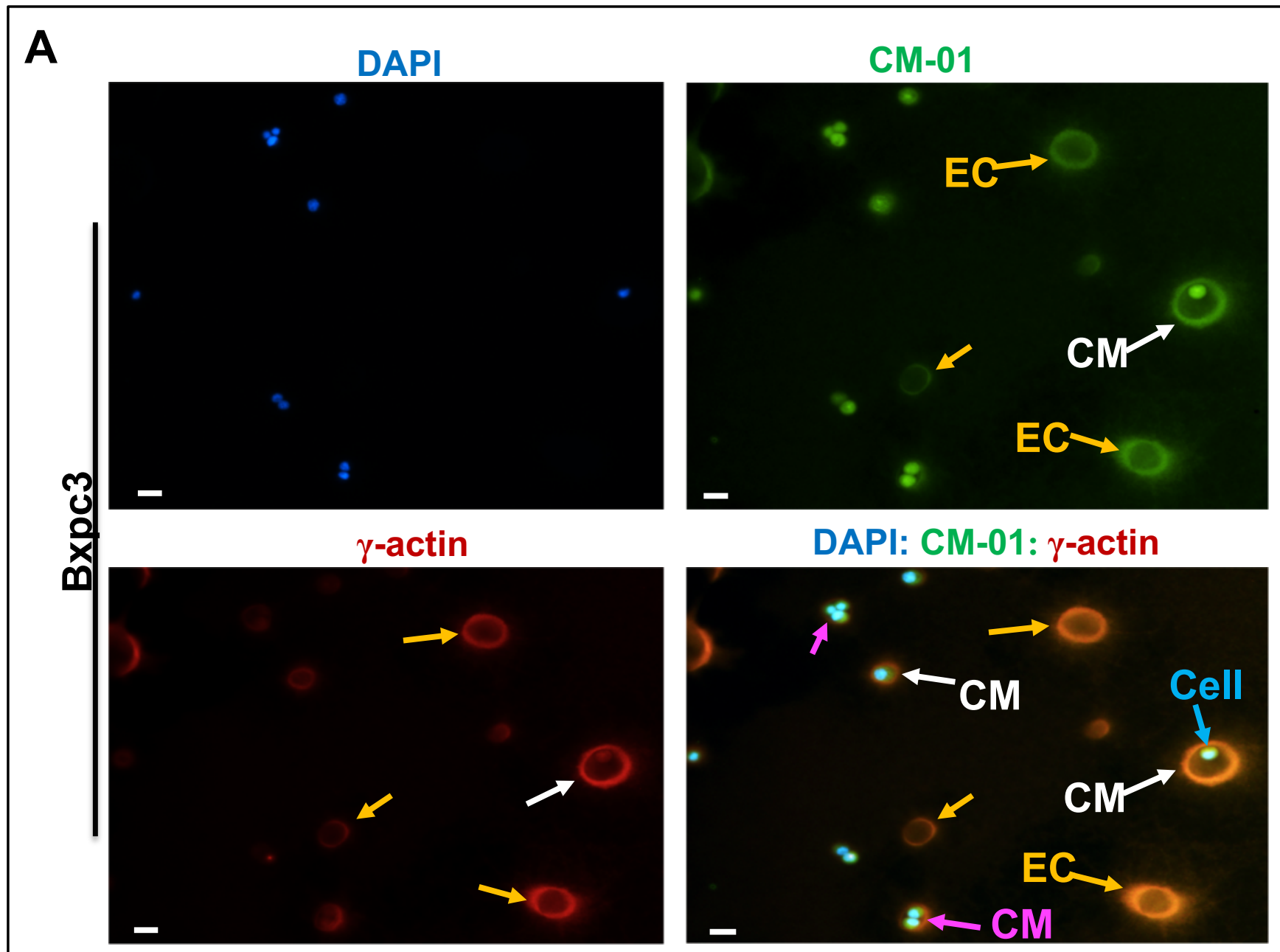

B

\section{Clinical thyroid cancer}

$\frac{1}{0}$ 


\section{Fig.S2}

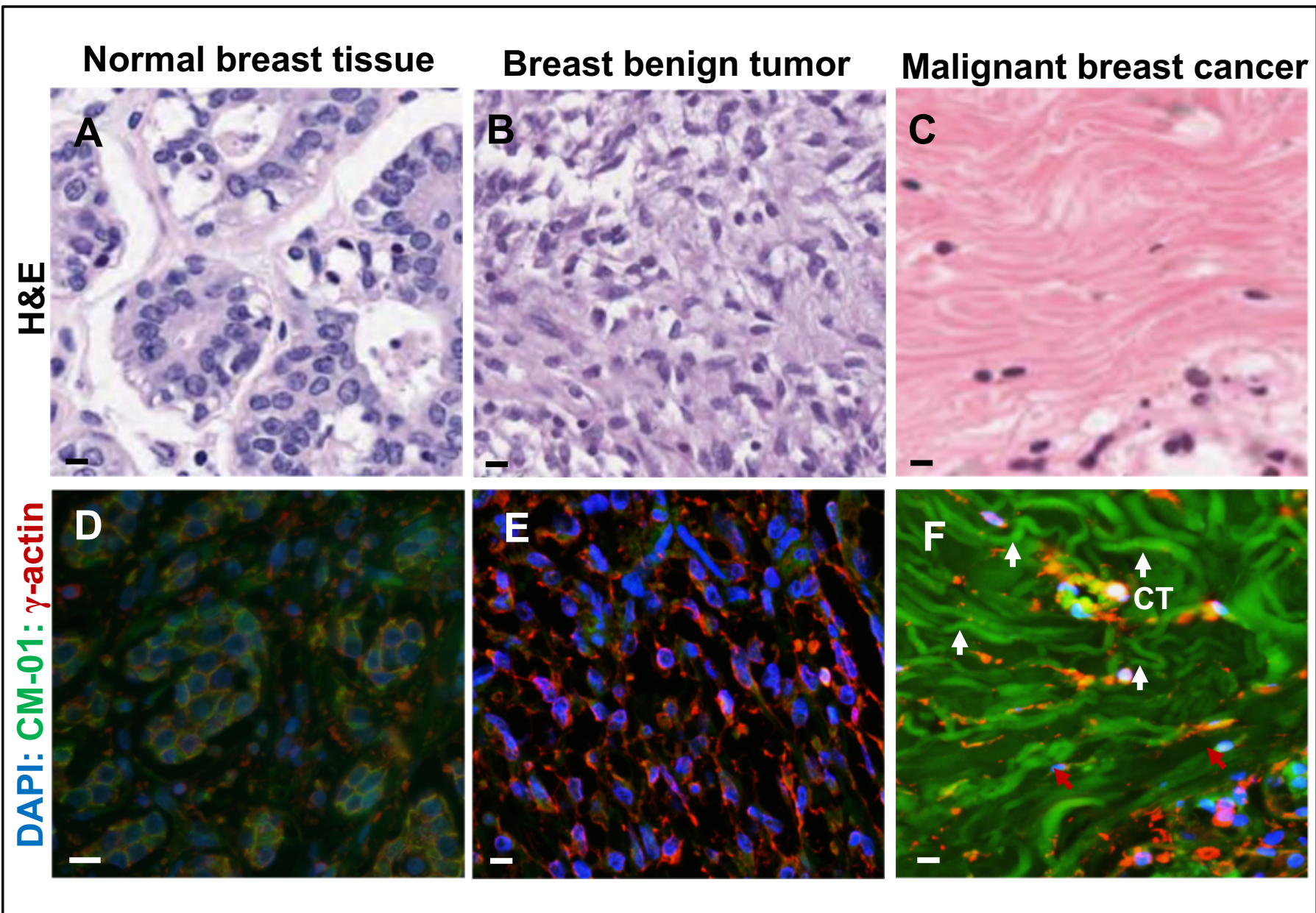




\section{Fig.S3}

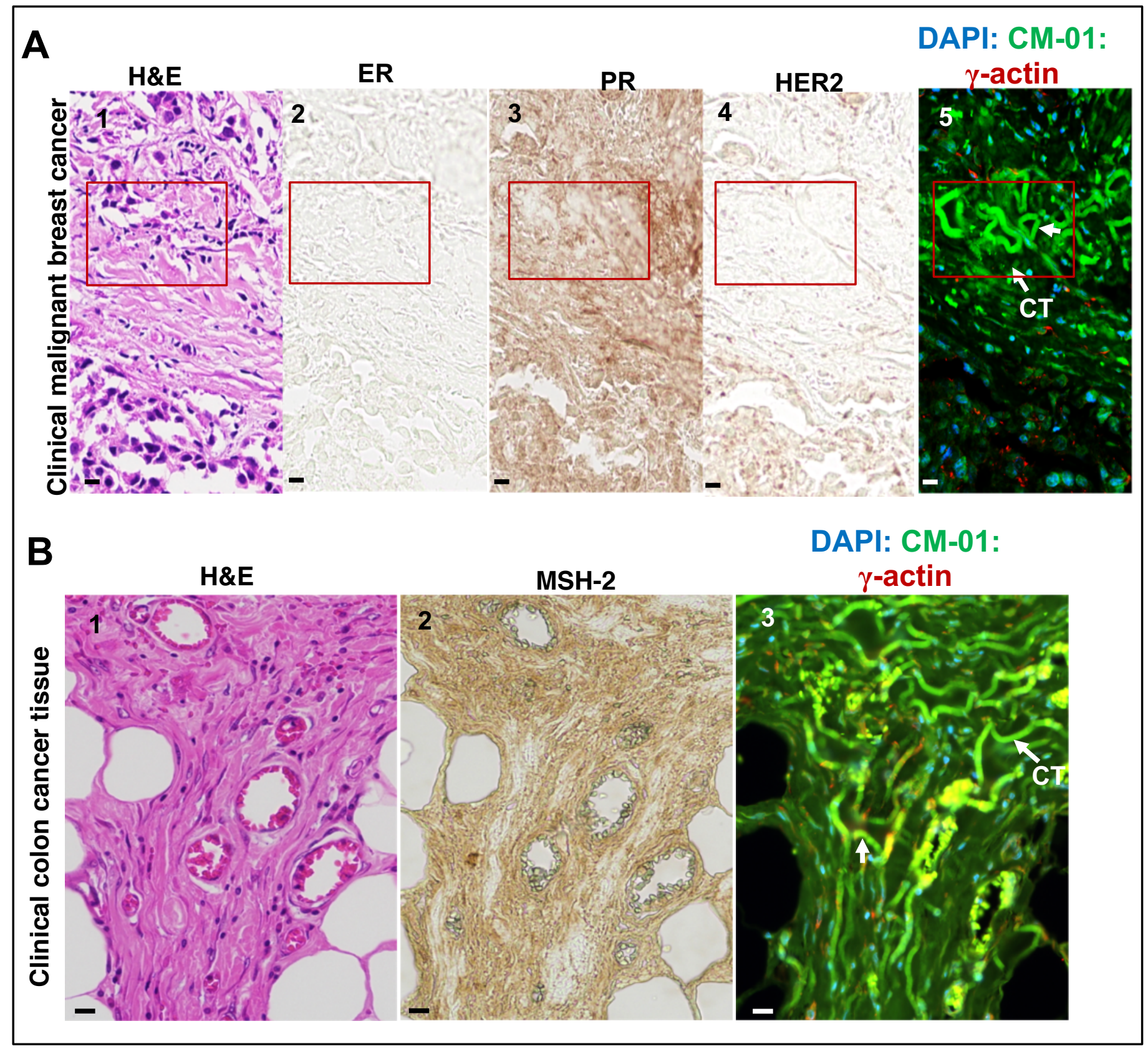


Fig.S4

was not certified by peer review) is the author/funder. All rights reserved. No reuse allowed without permission.

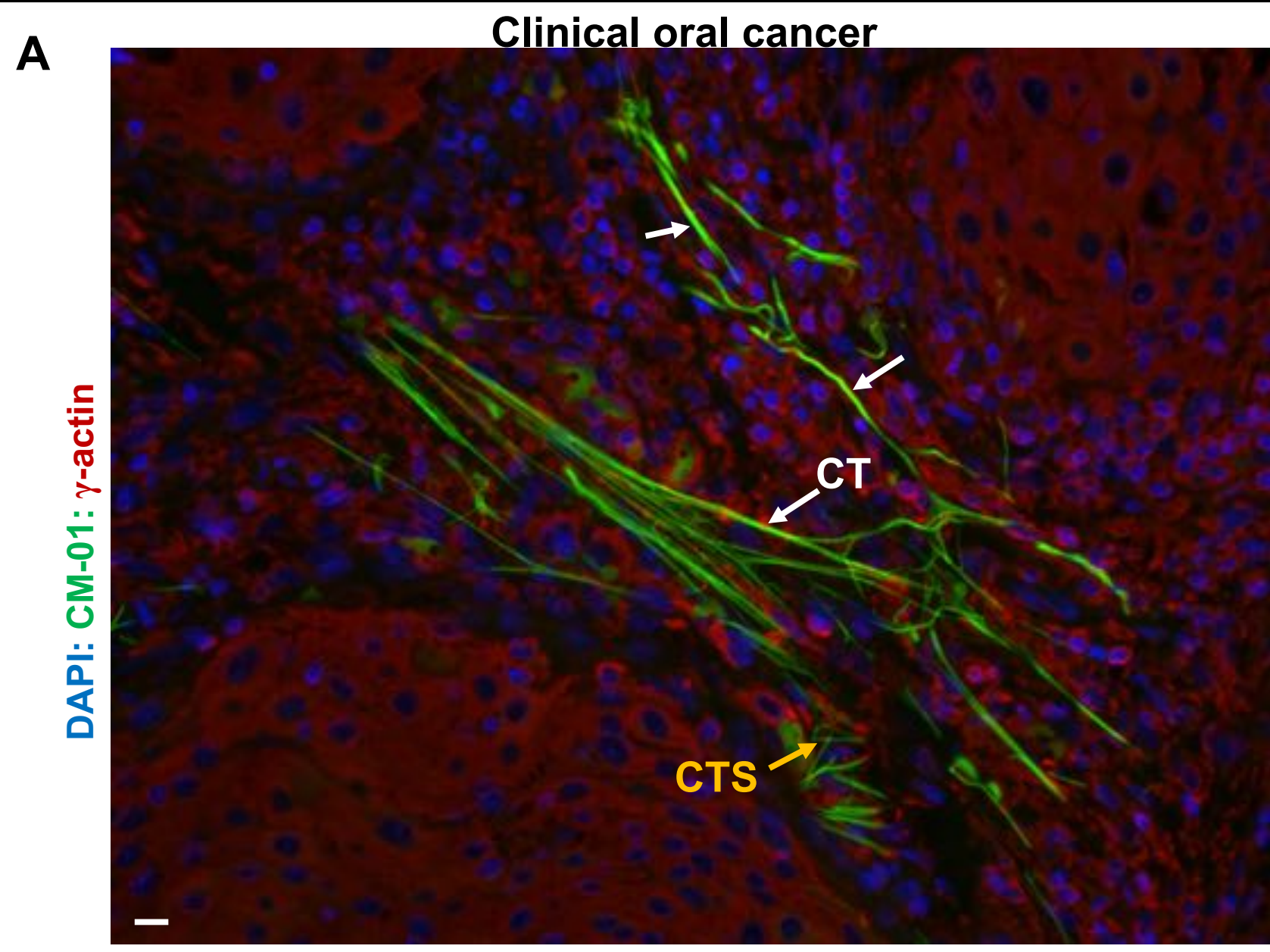

B

\section{Clinical thyroid cancer}

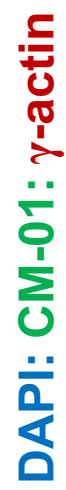

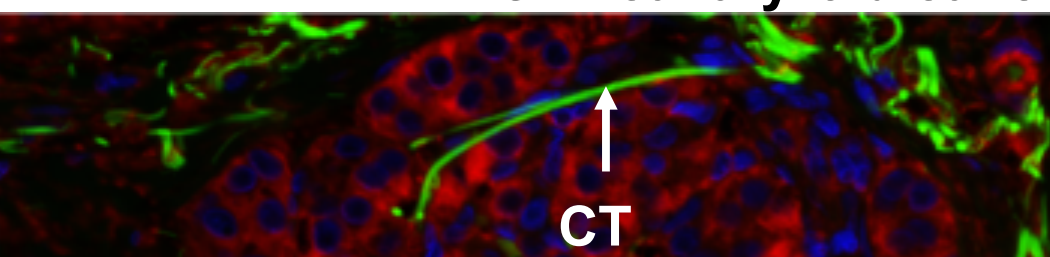

 
Fig.S5

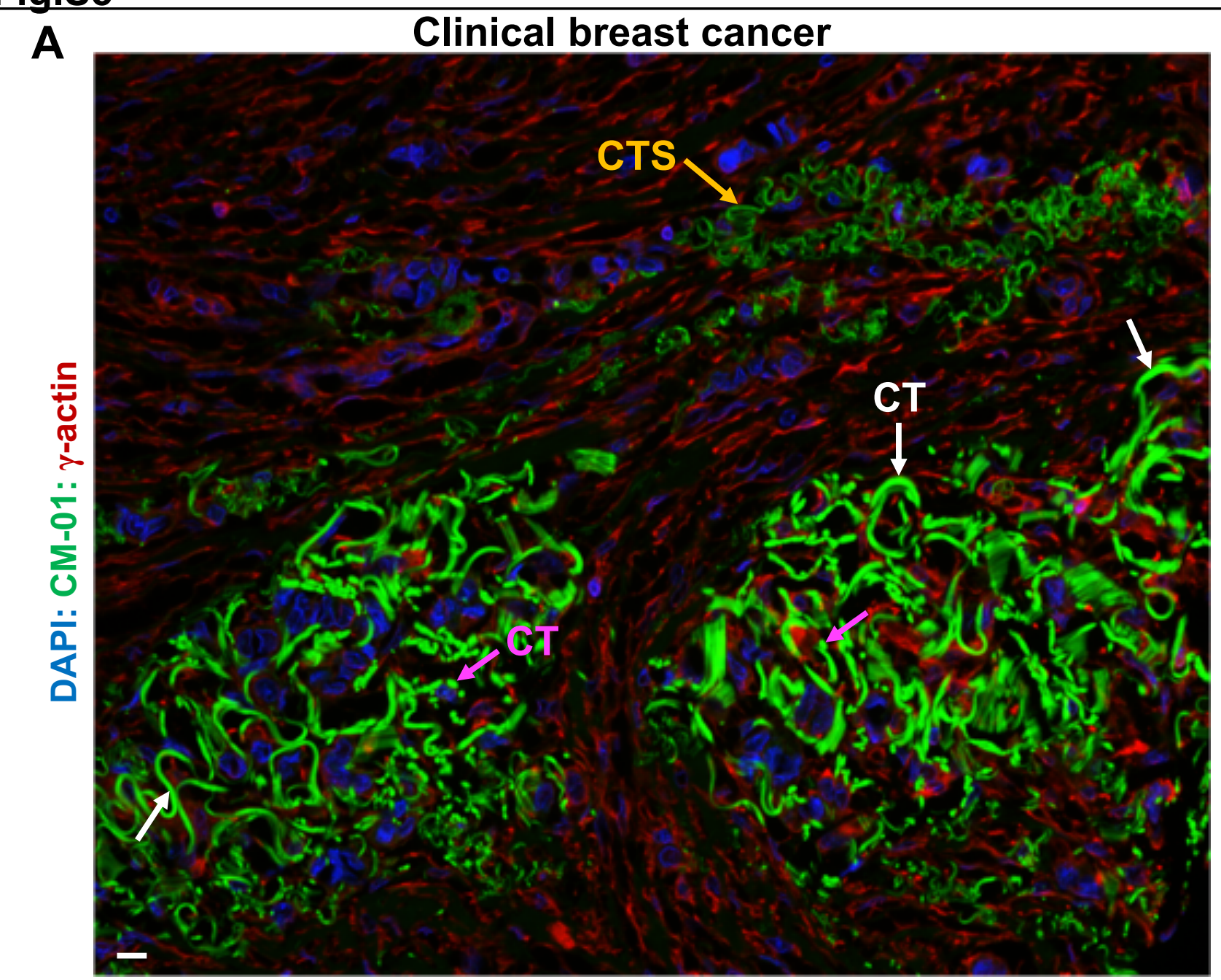

B

Clinical pancreas cancer

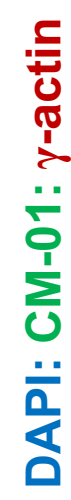

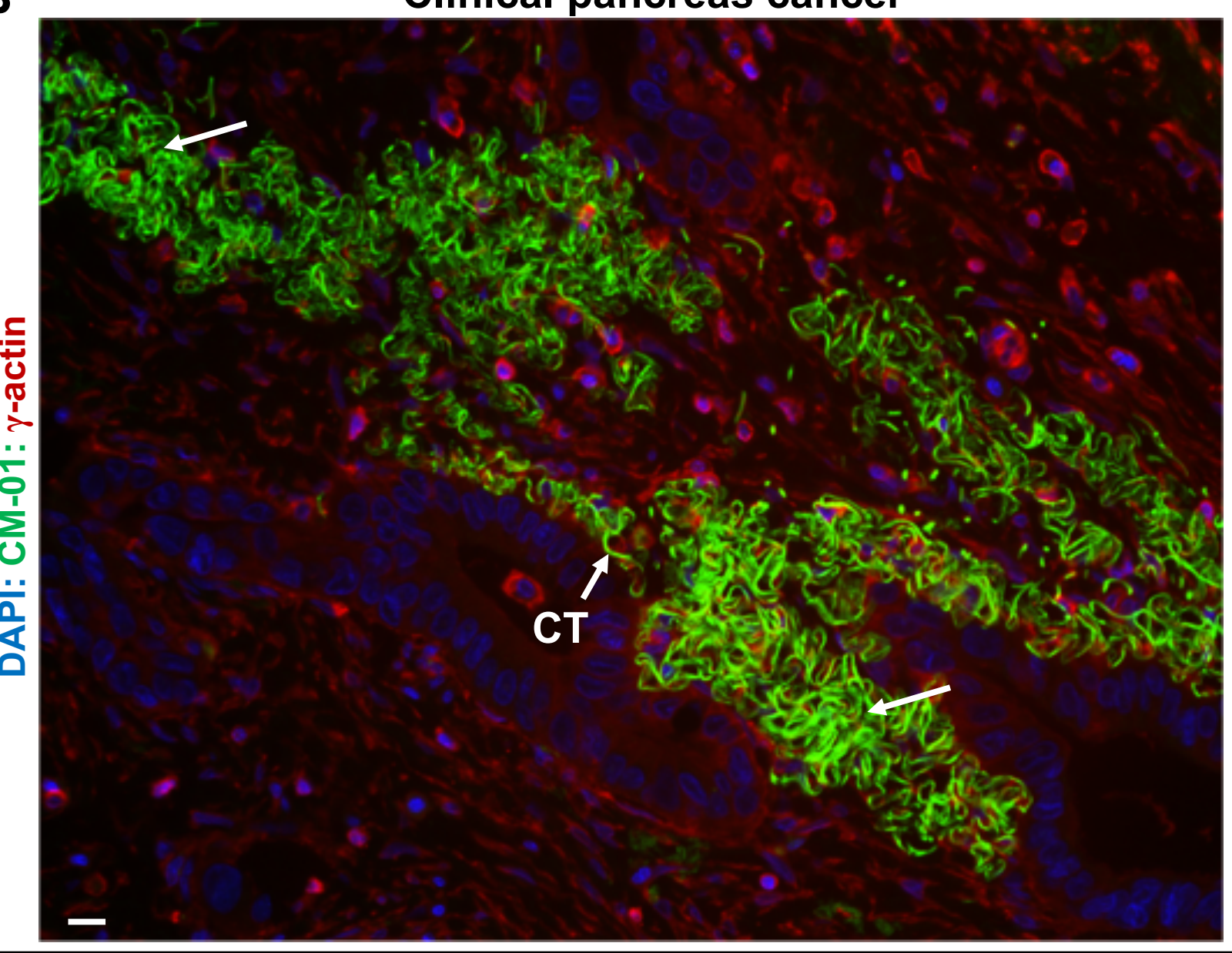




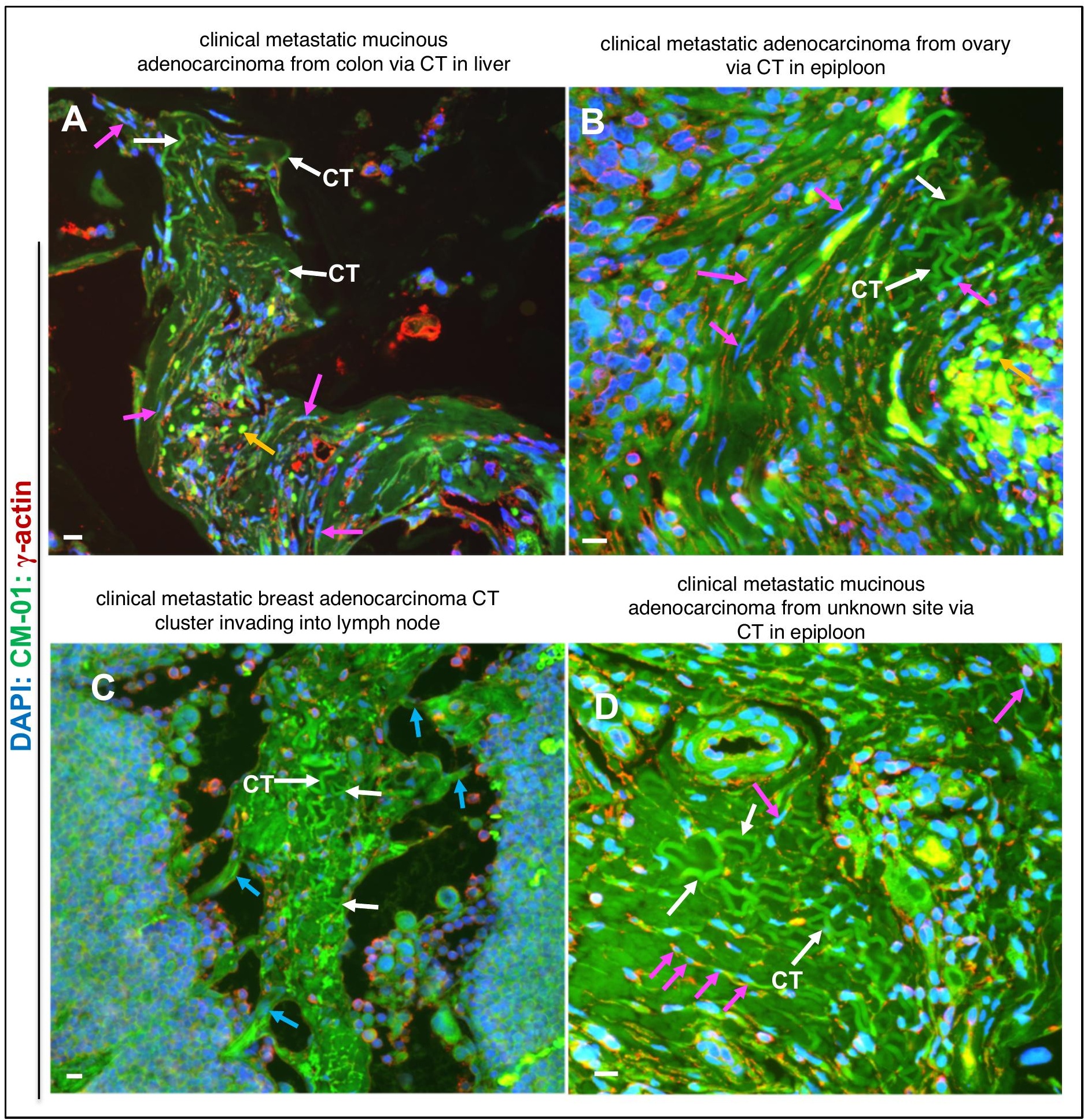


Fig. 57

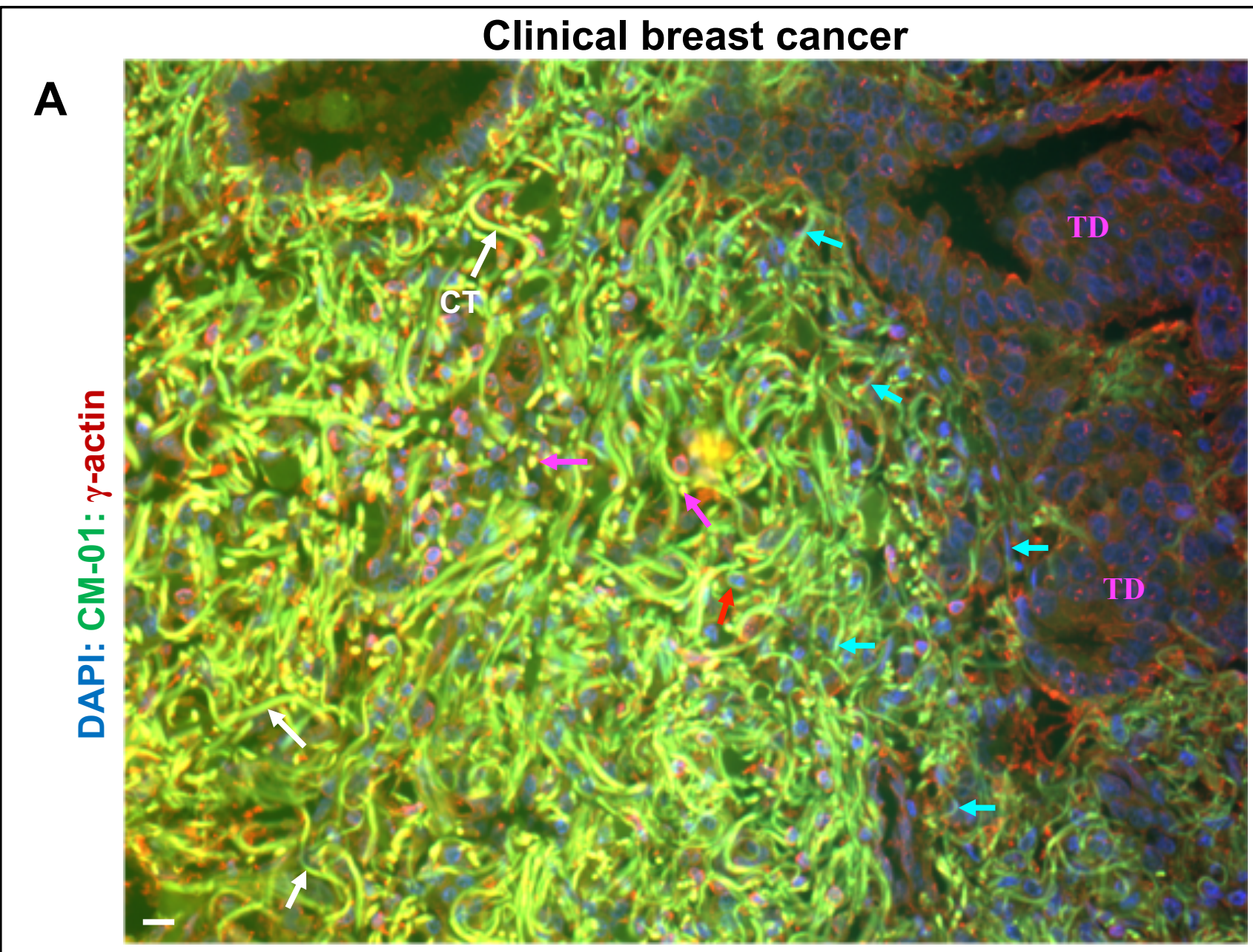

B

\section{Clinical breast cancer}

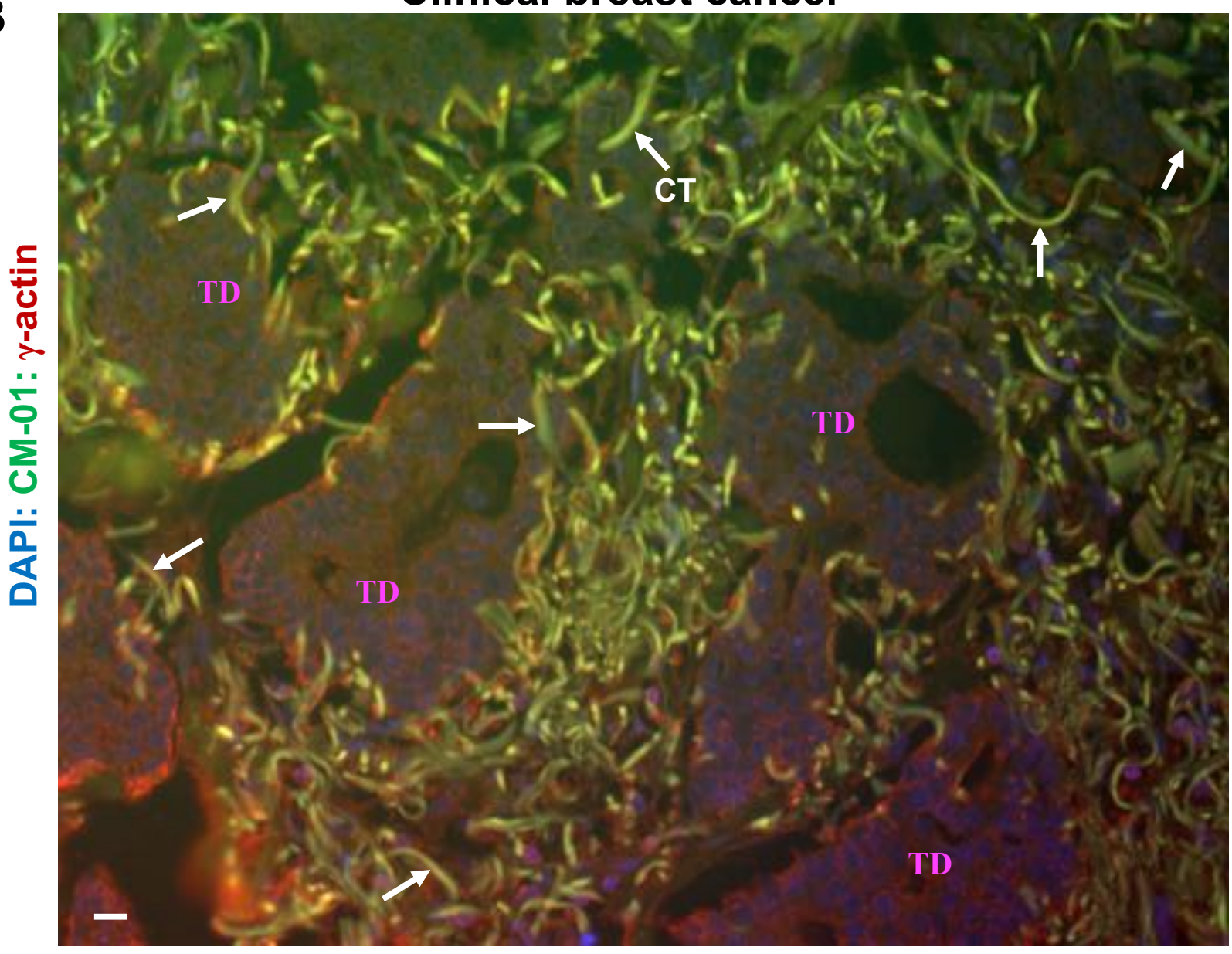




\section{Fig.S8}

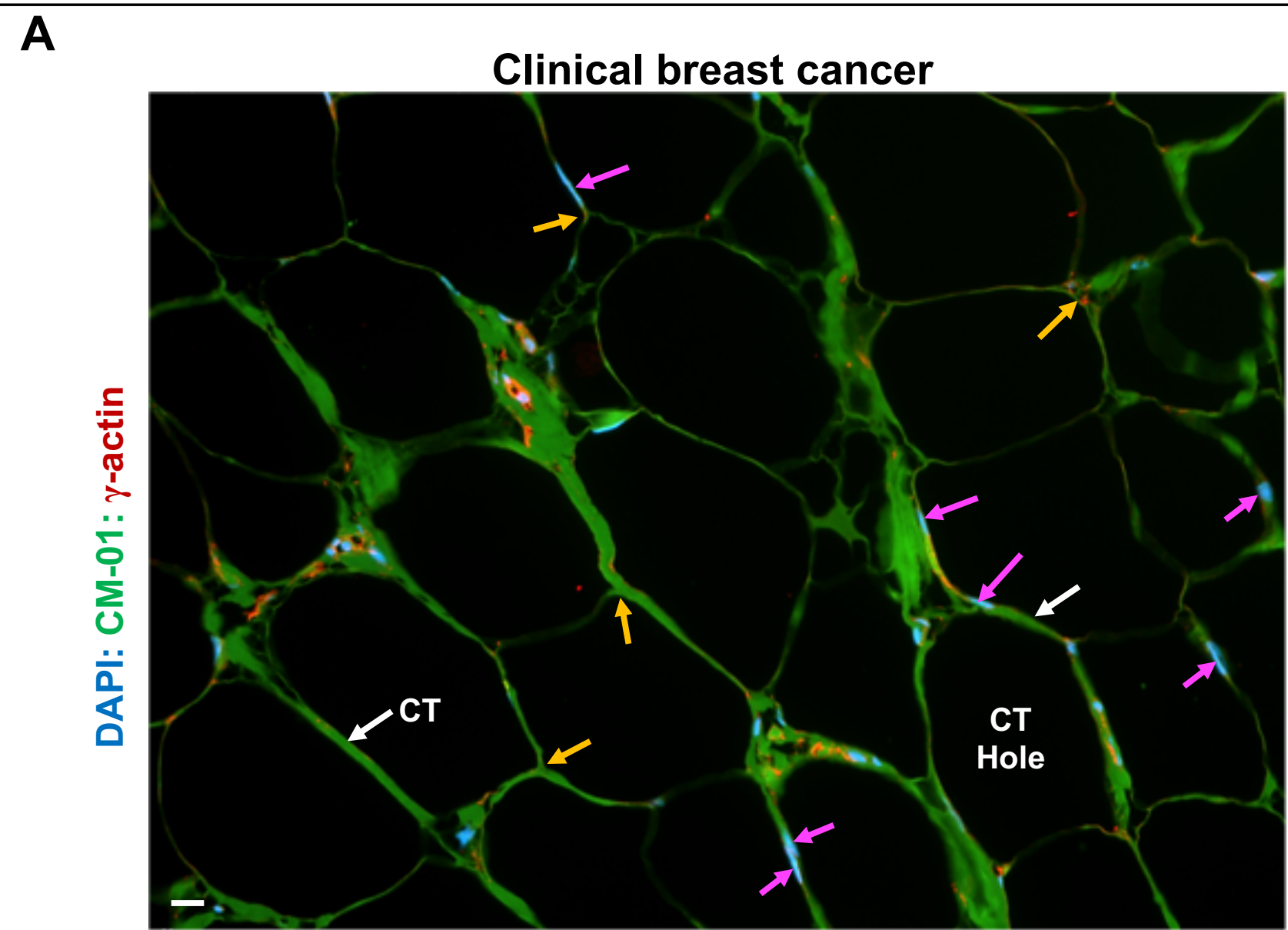

B

Clinical colon cancer

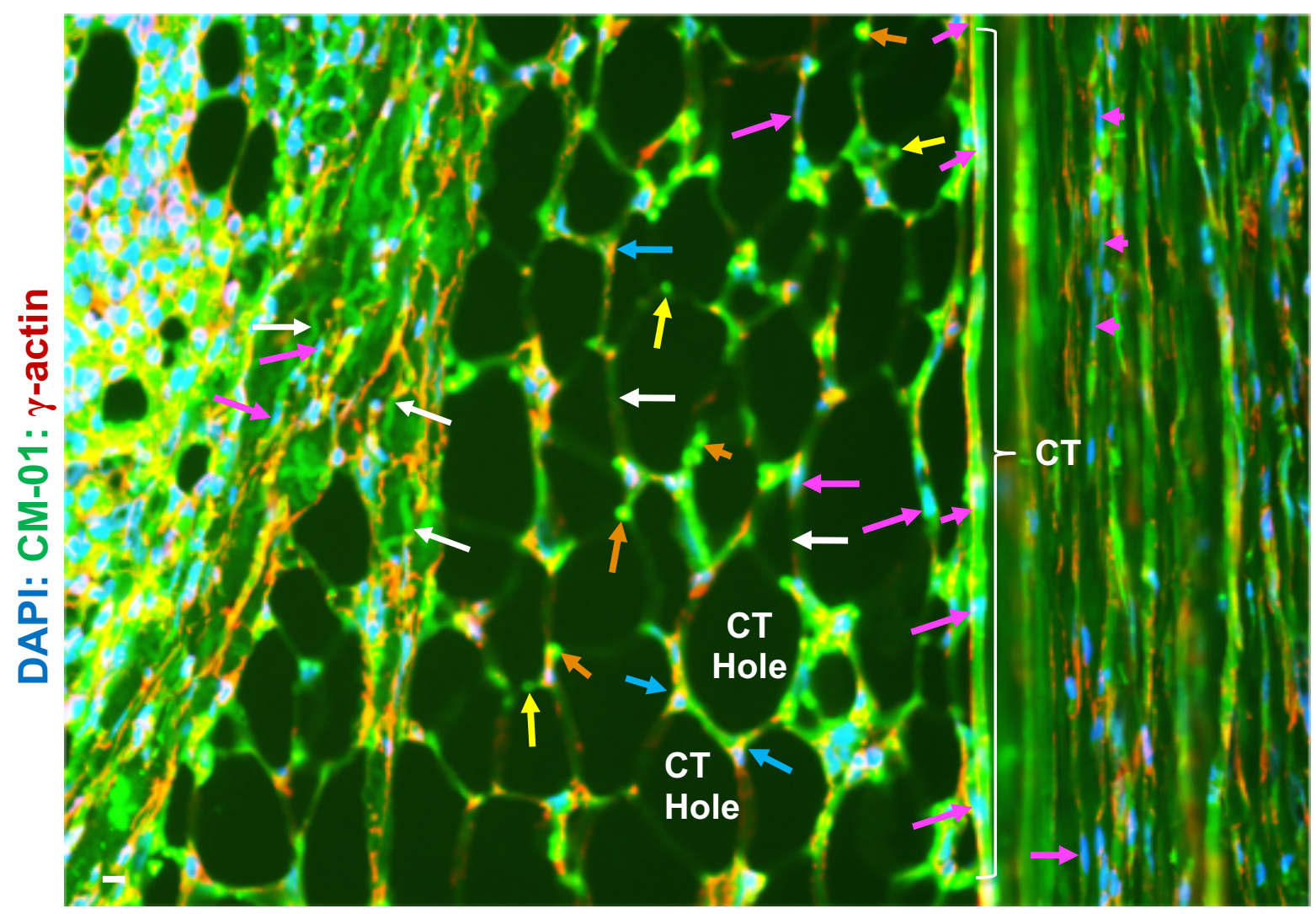




\section{Fig.S9}

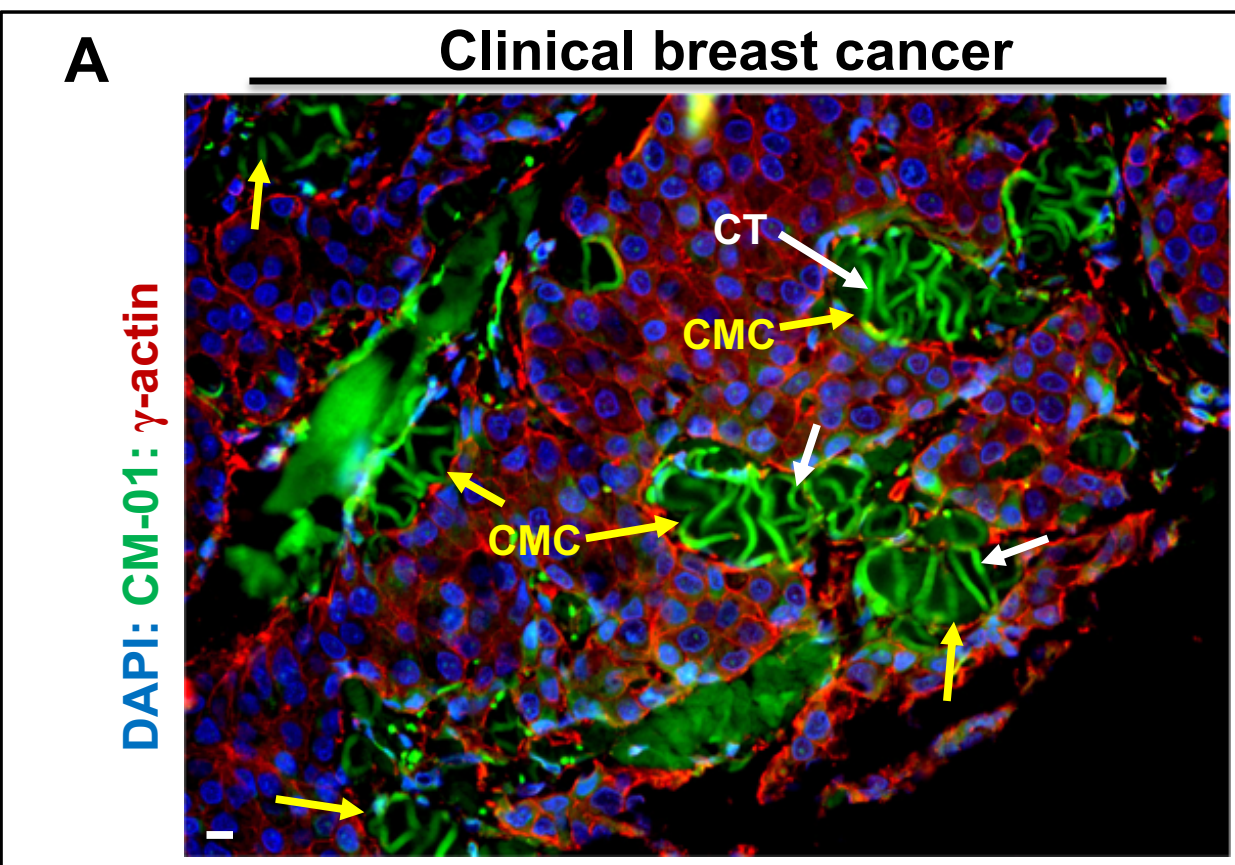

B

C
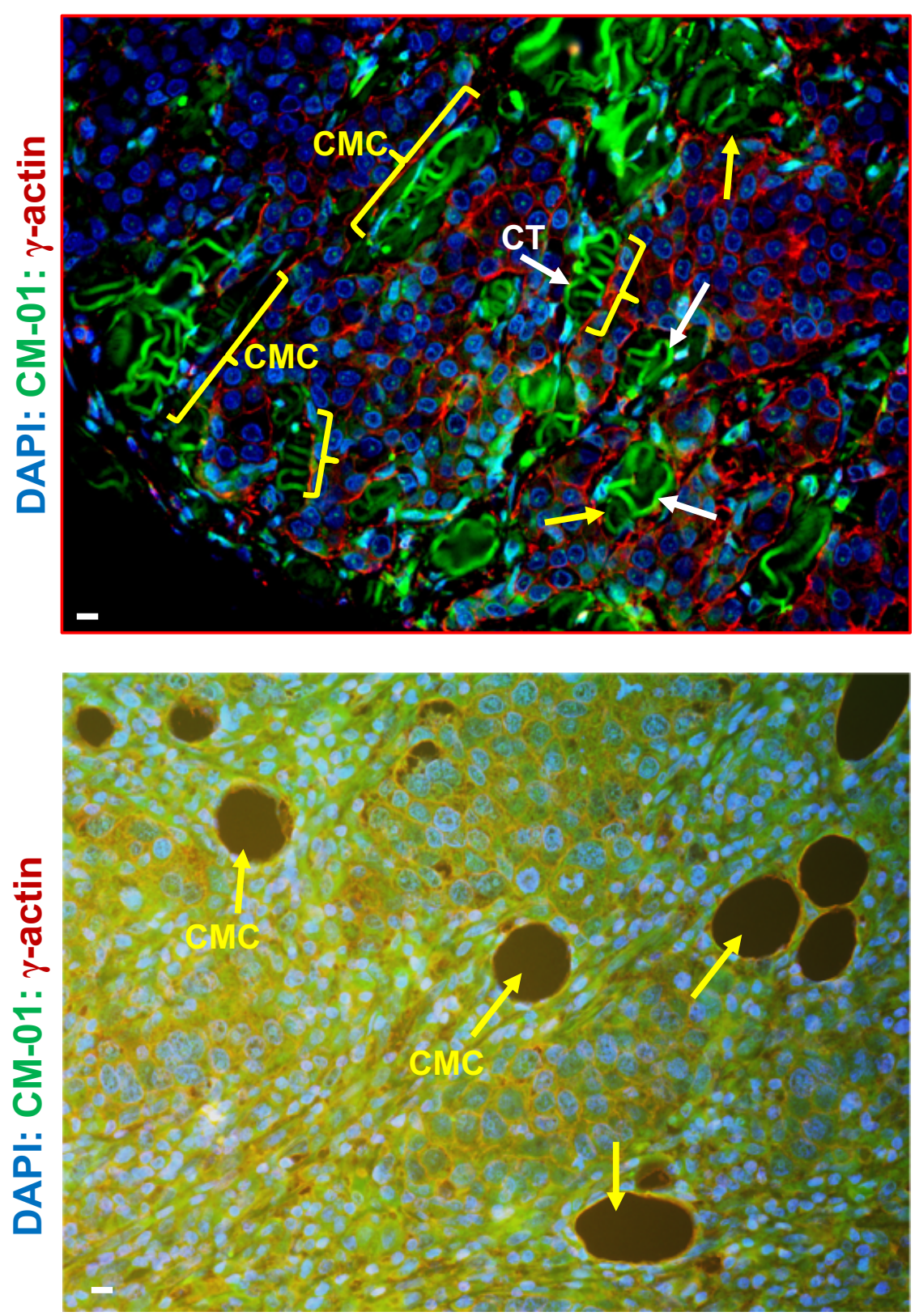


\section{Fig.S10}

A

\section{Clinical breast cancer}

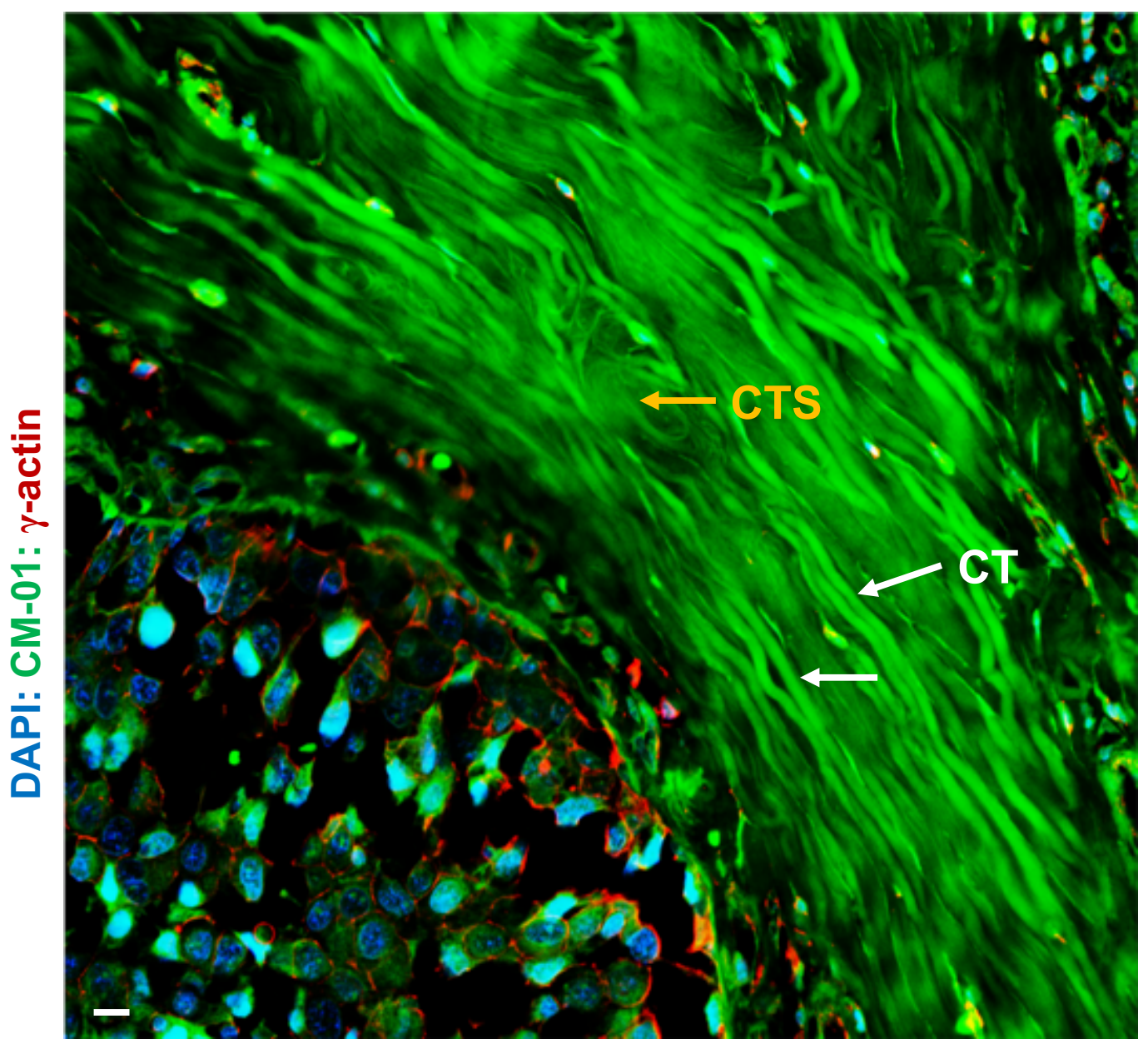

B

\section{Clinical breast cancer}

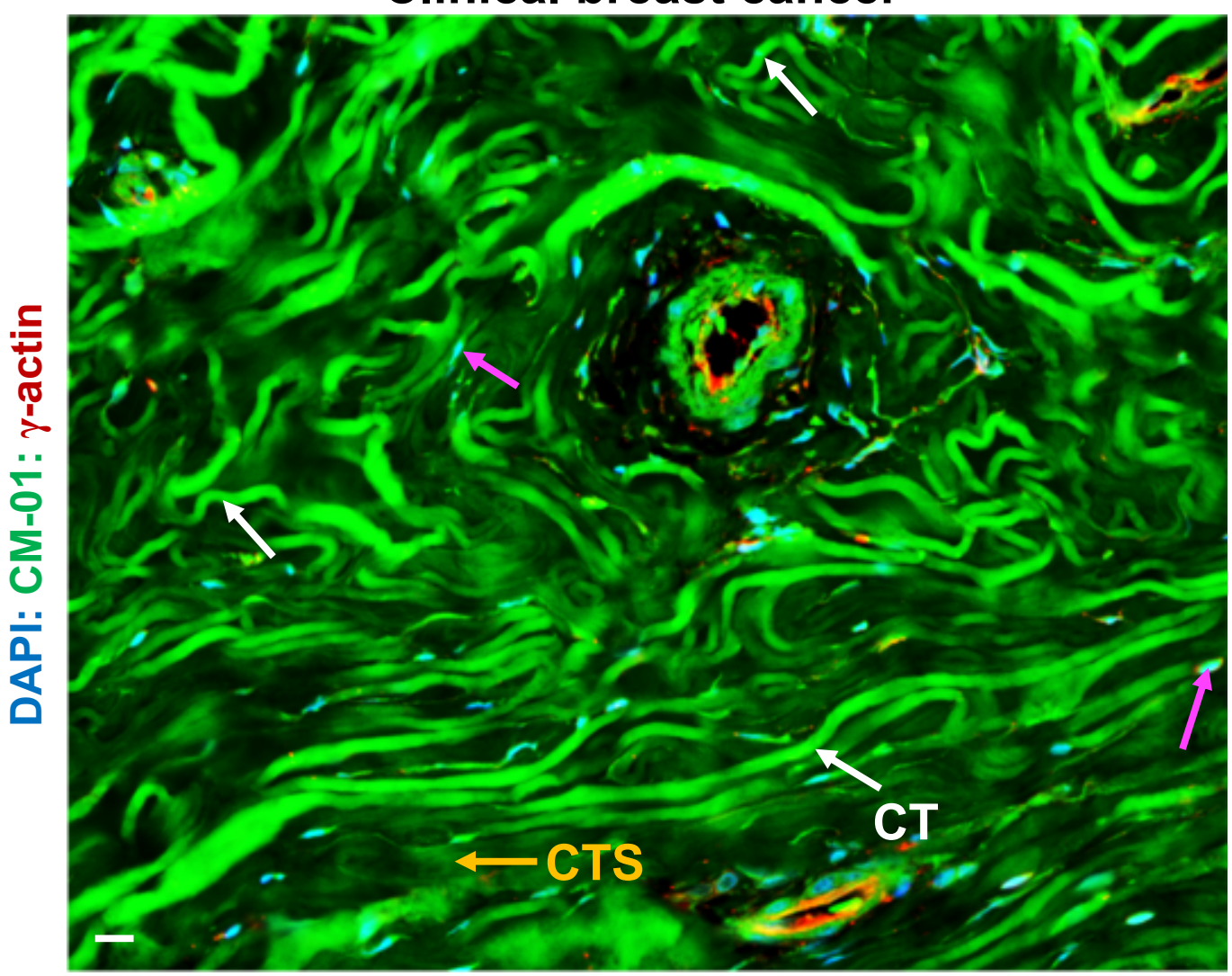




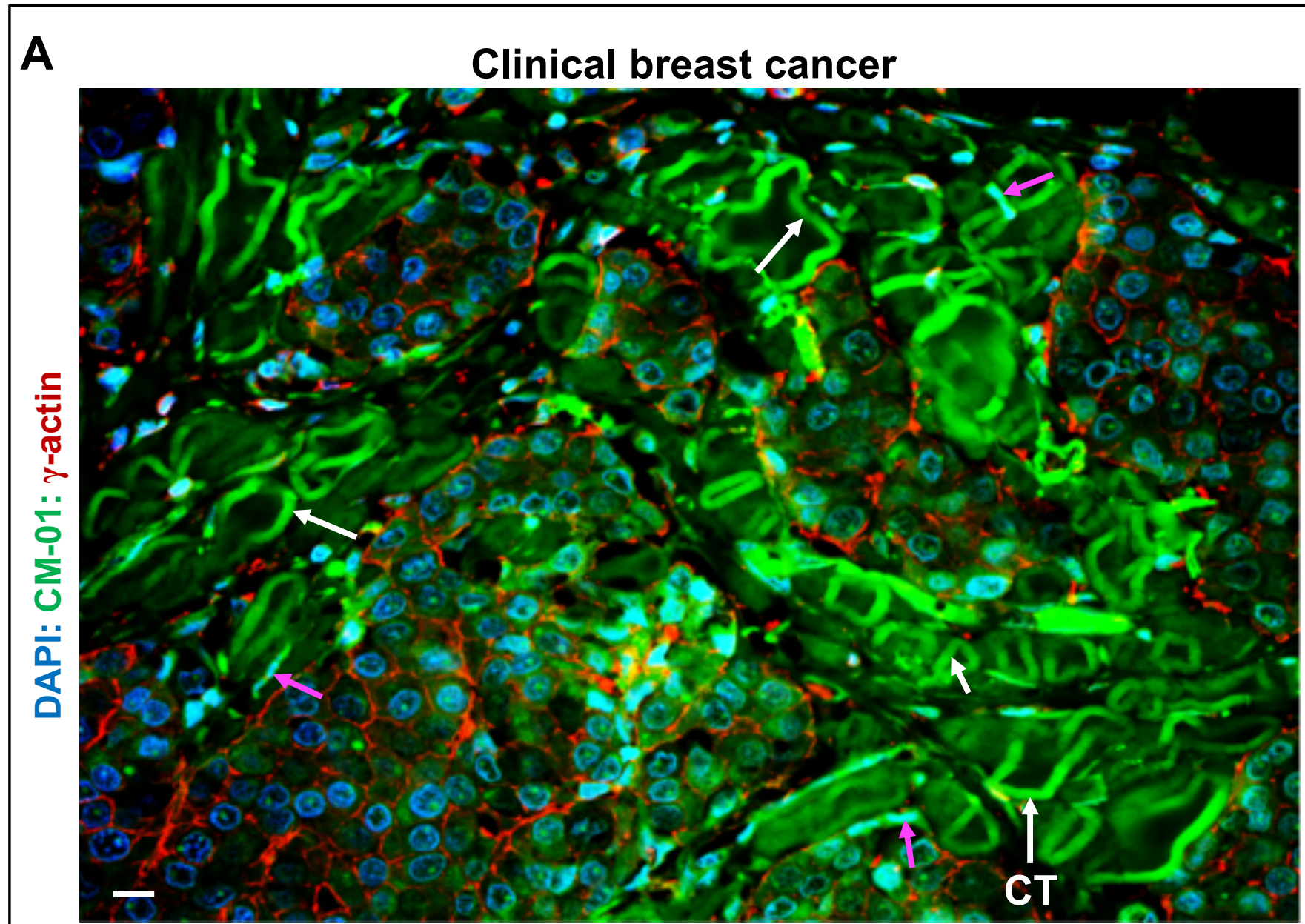

B

\section{Clinical breast cancer}

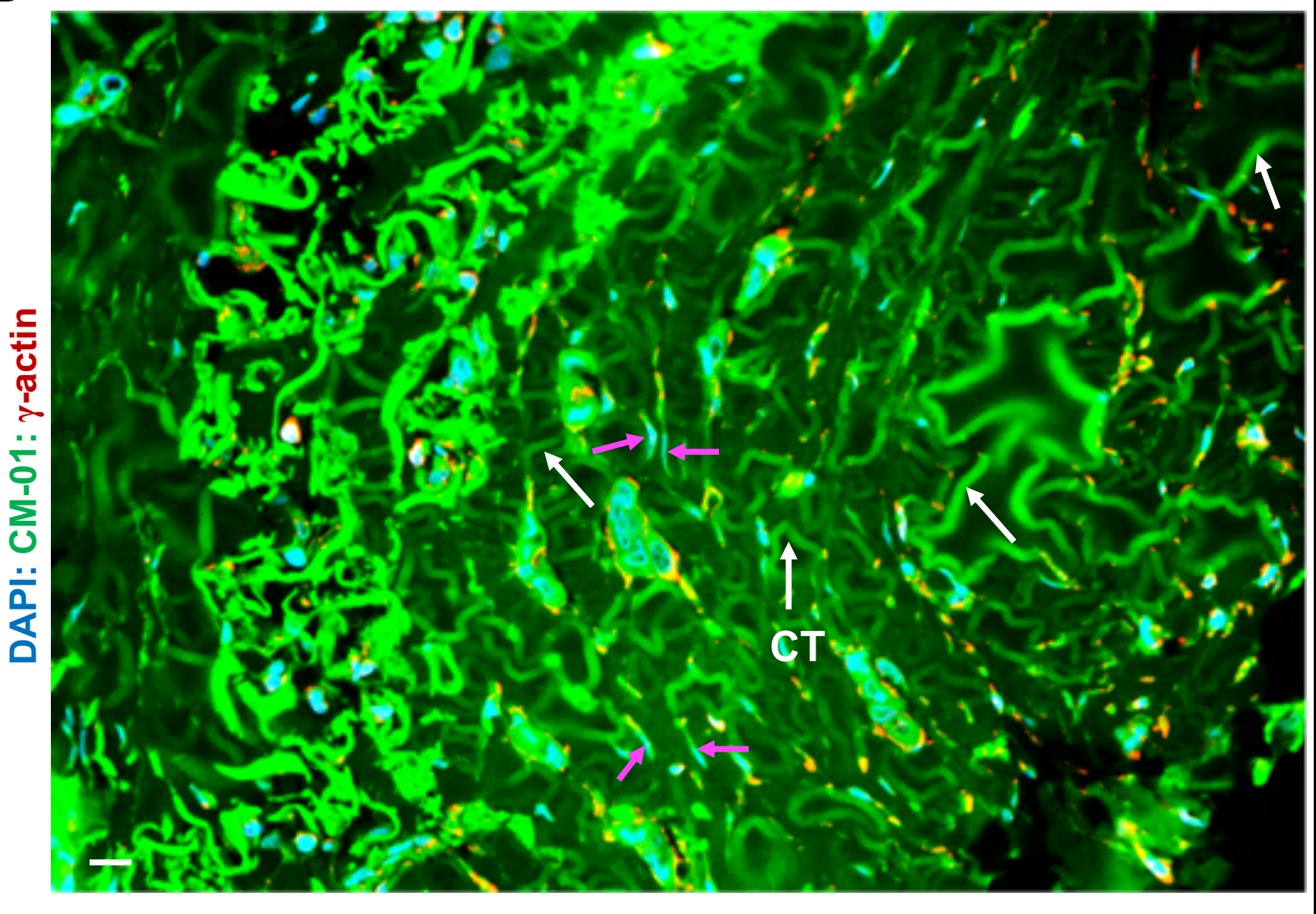




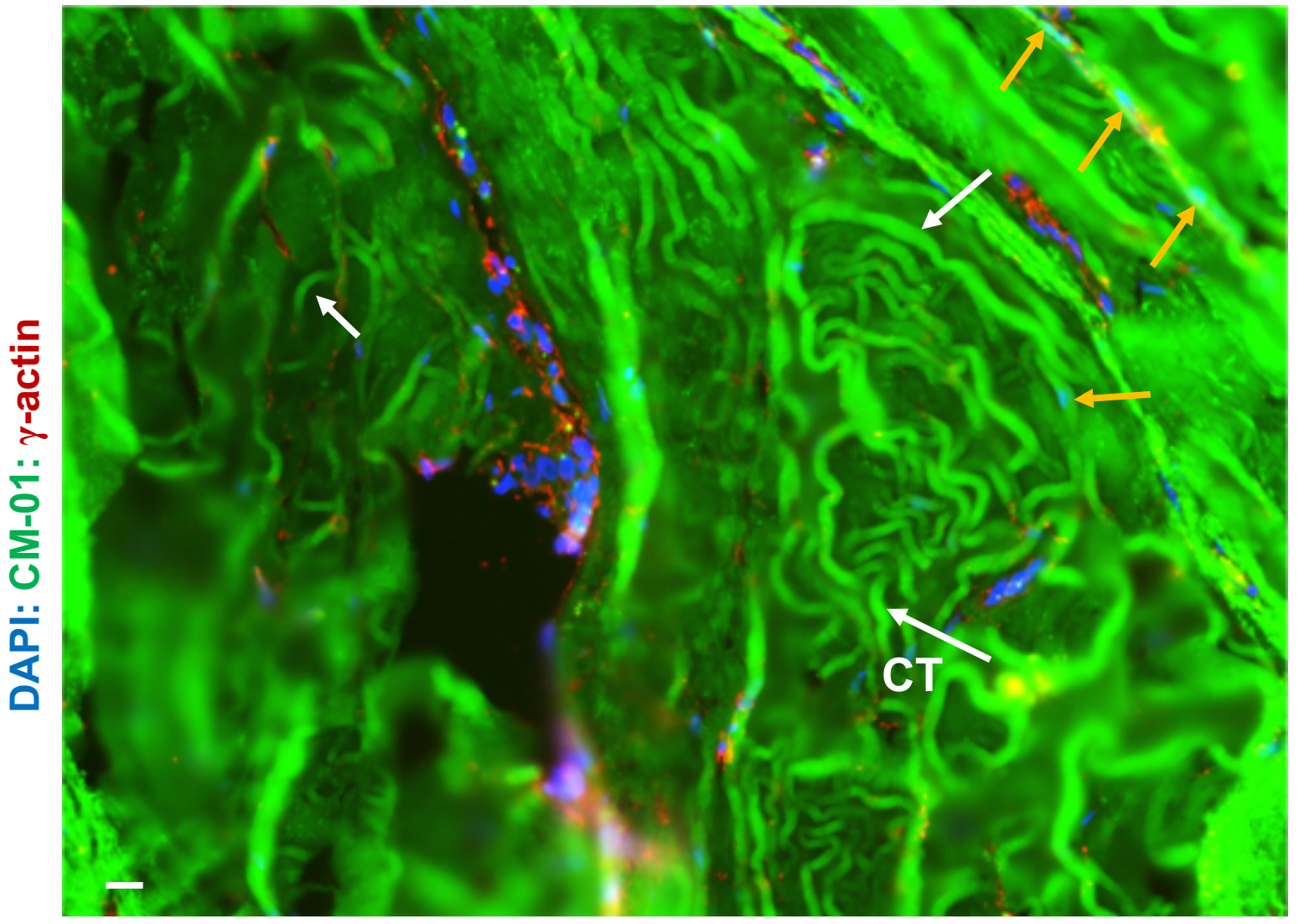

B

\section{Clinical breast cancer}

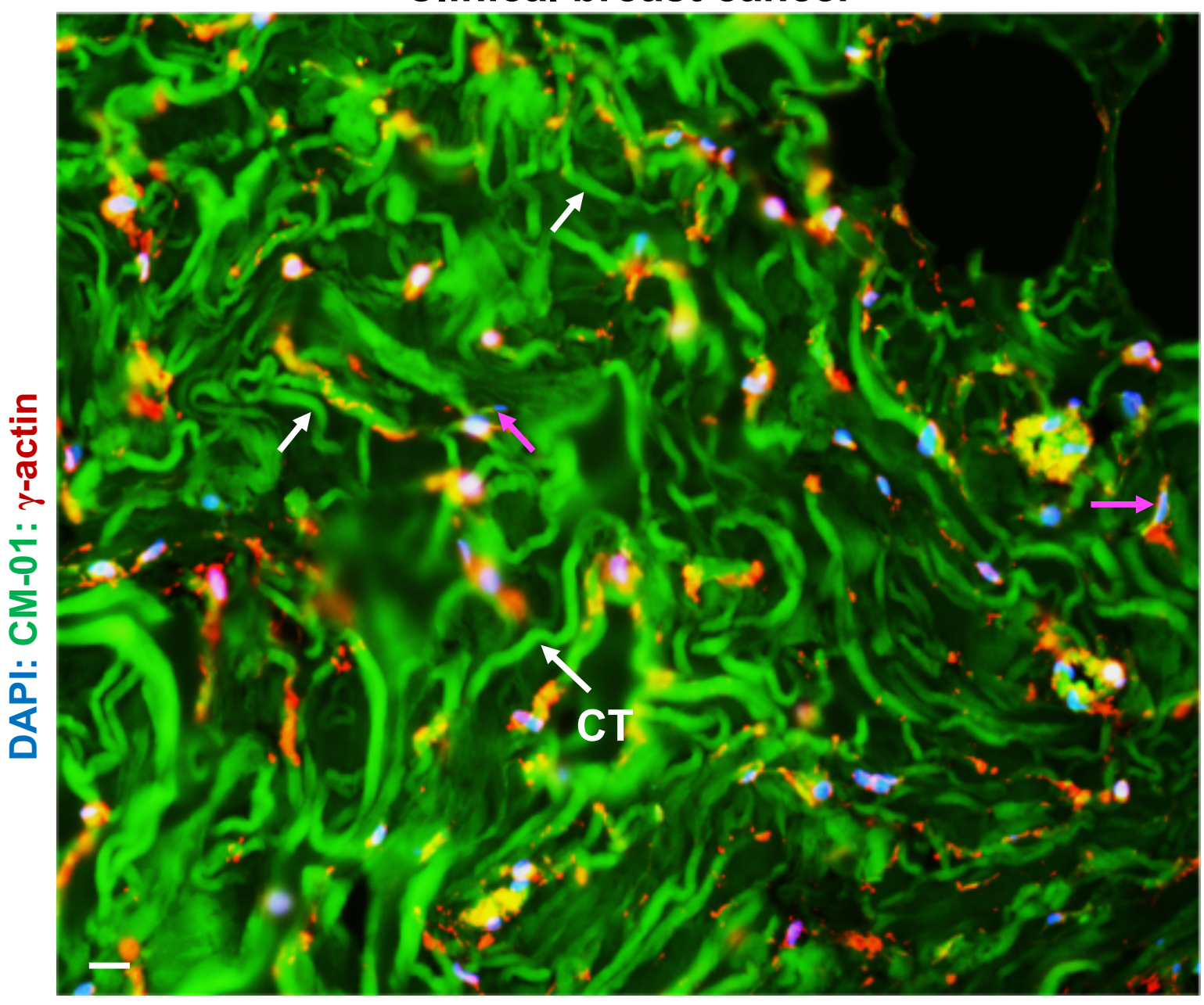




\section{Fig.S13}

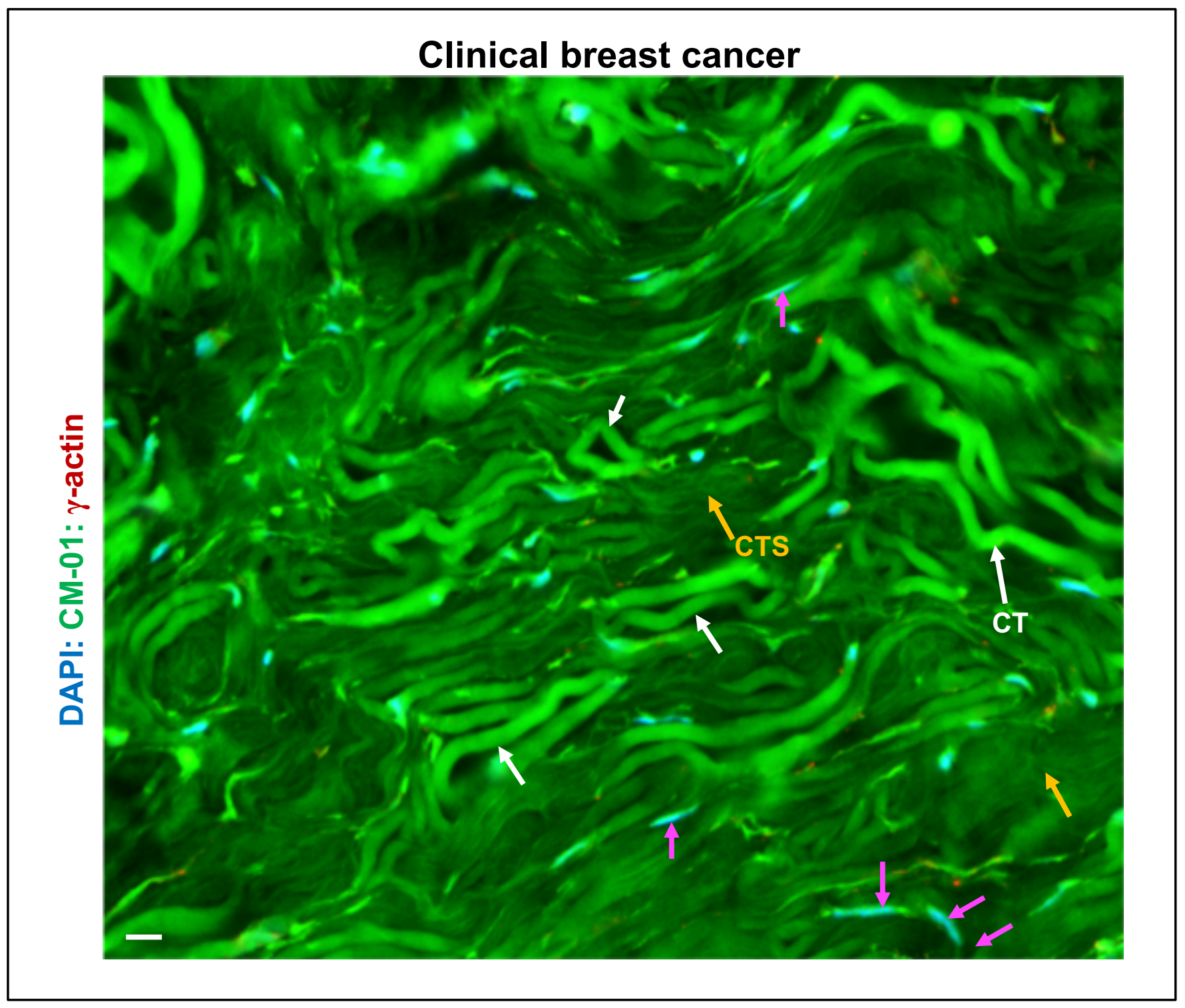


Fig.S14

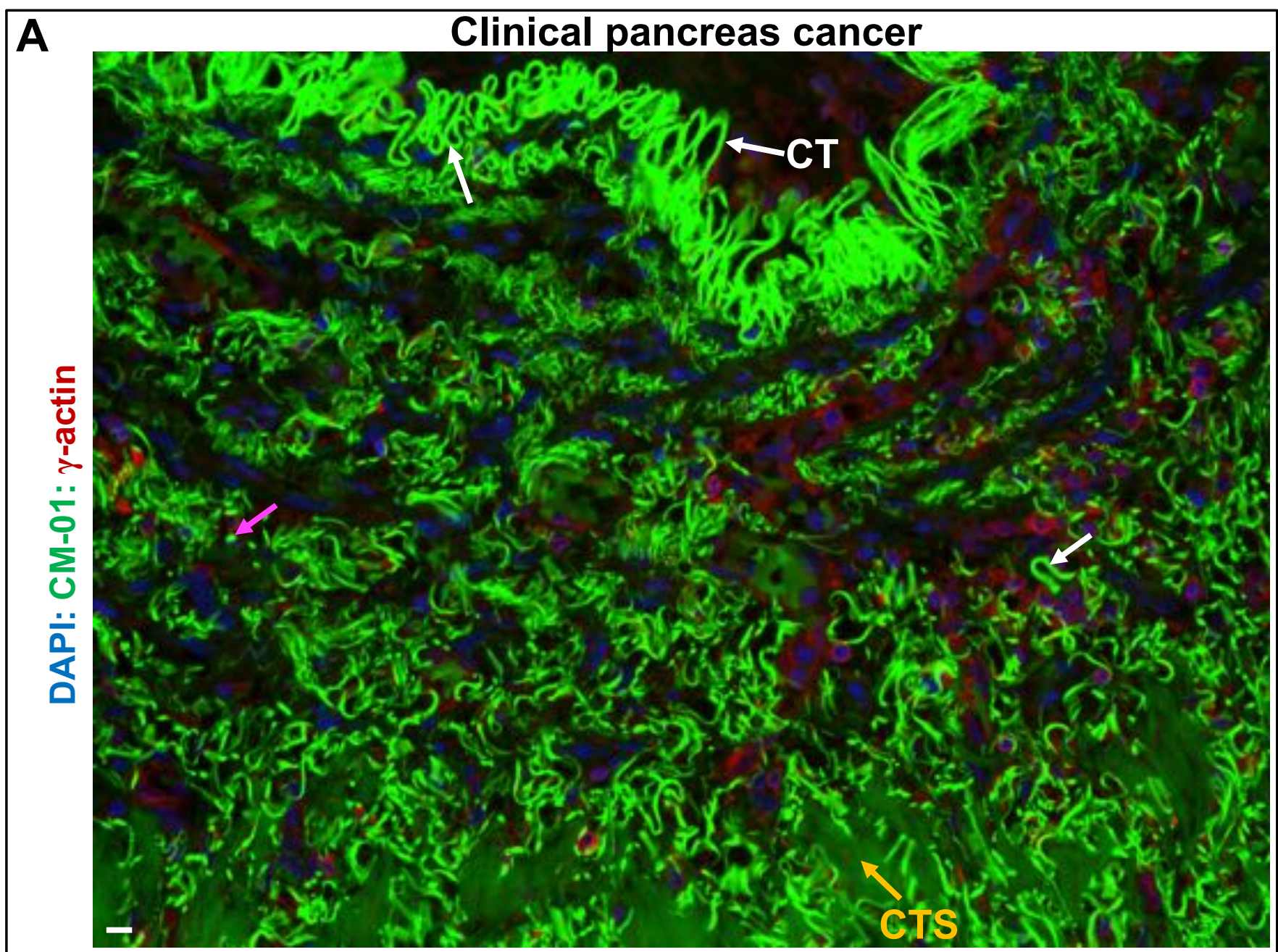

B

Clinical lung cancer

들

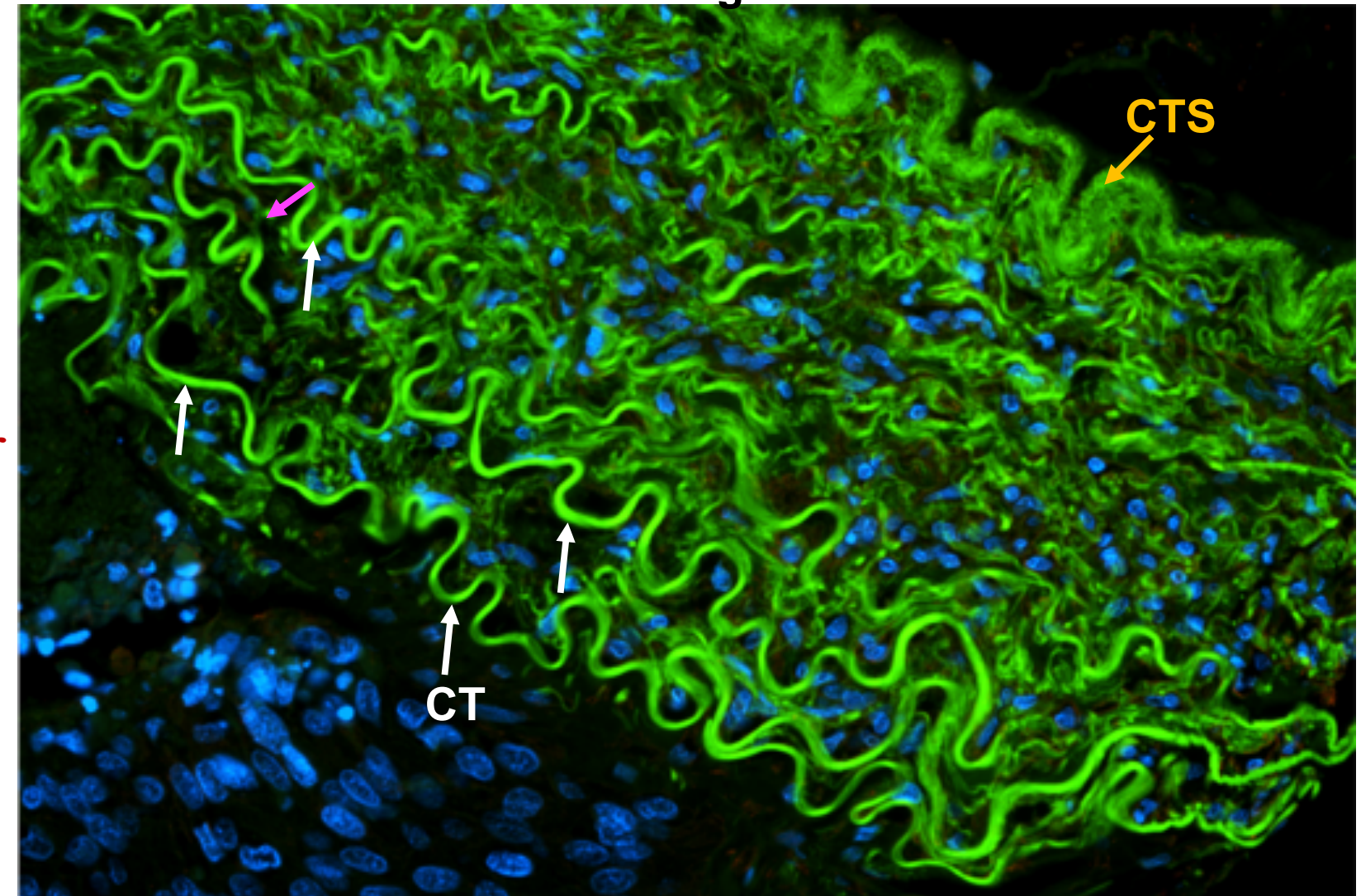




\section{Fig.S15}

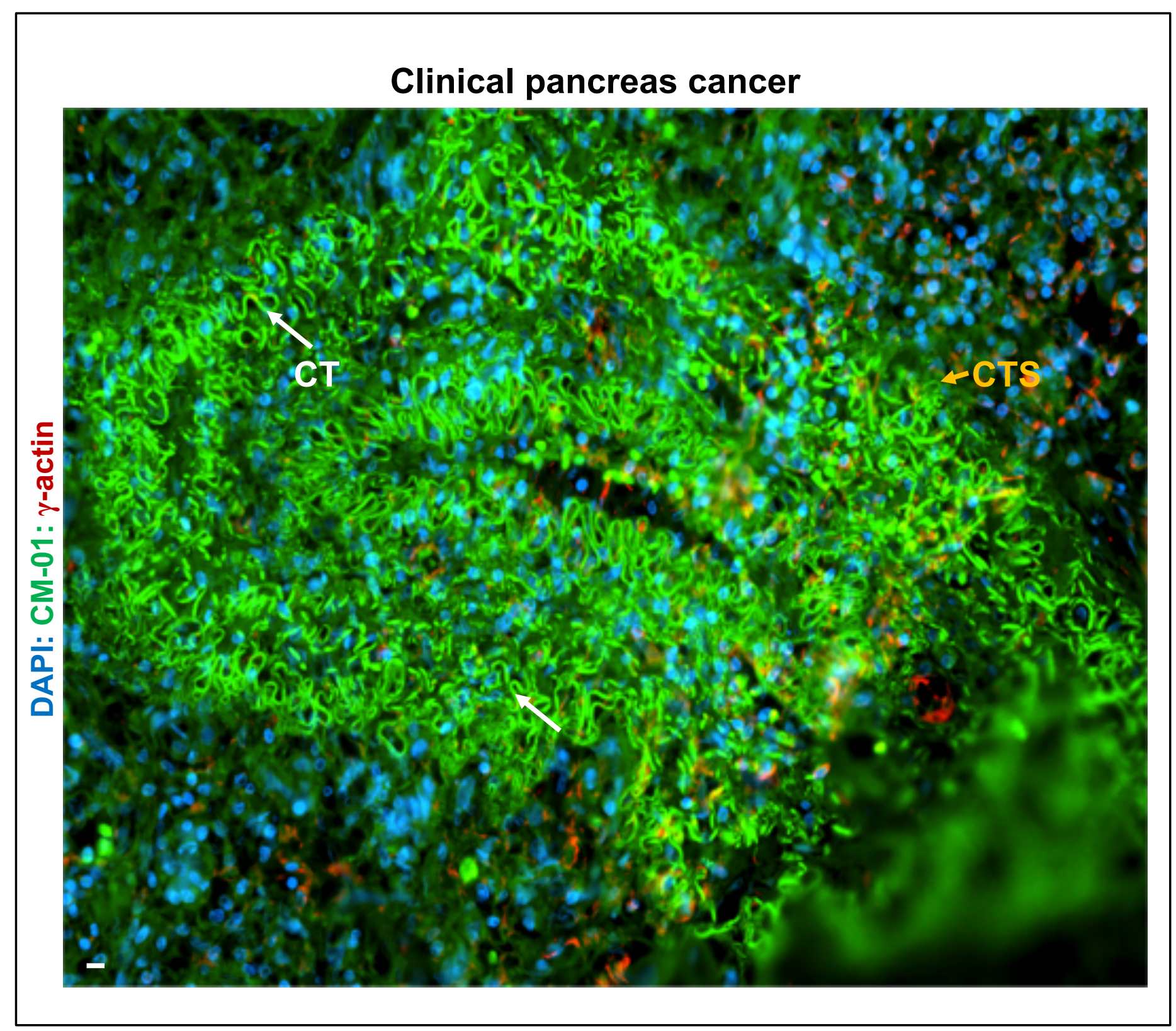




\section{Fig.S16}

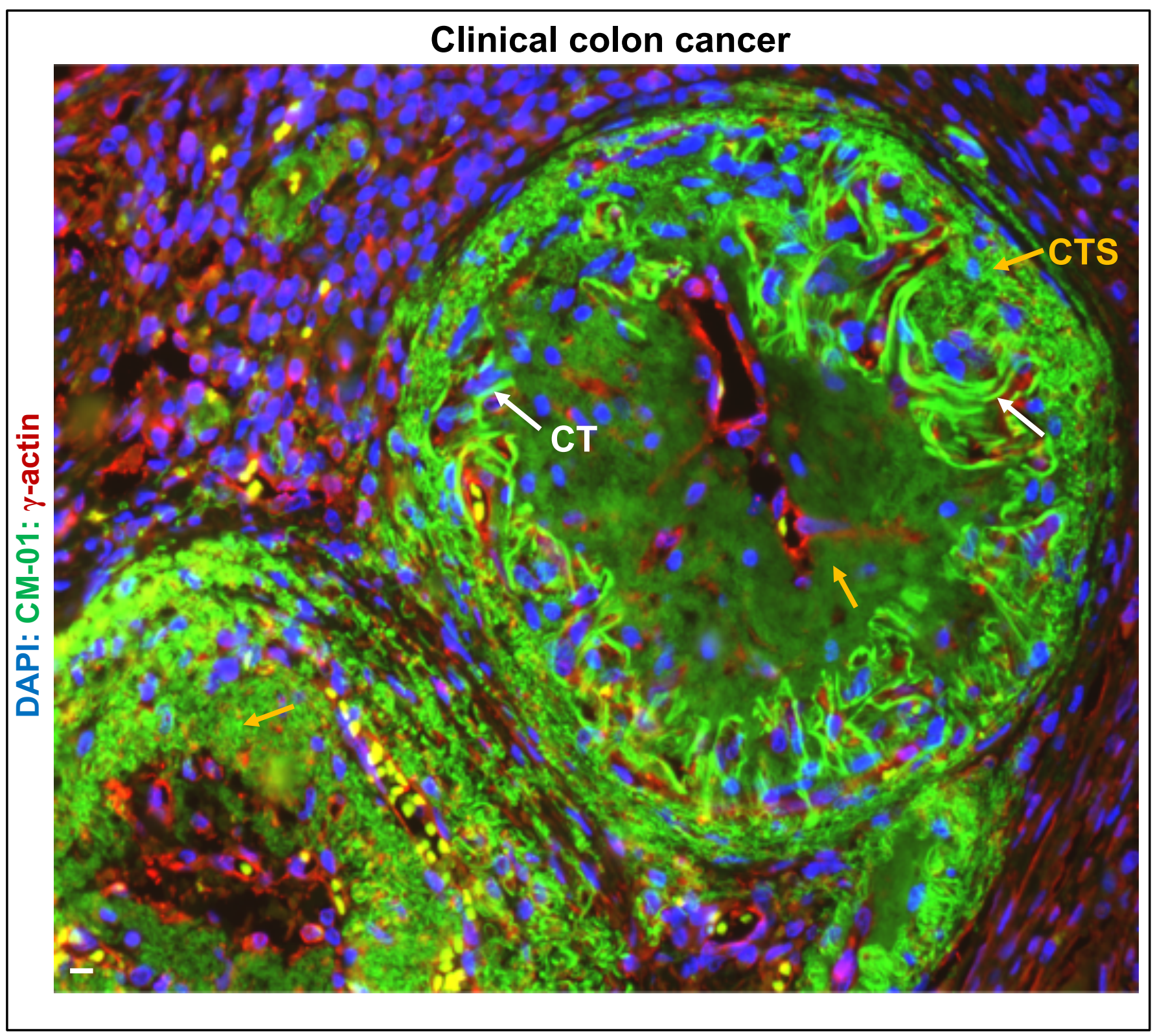




\section{Fig.S18}

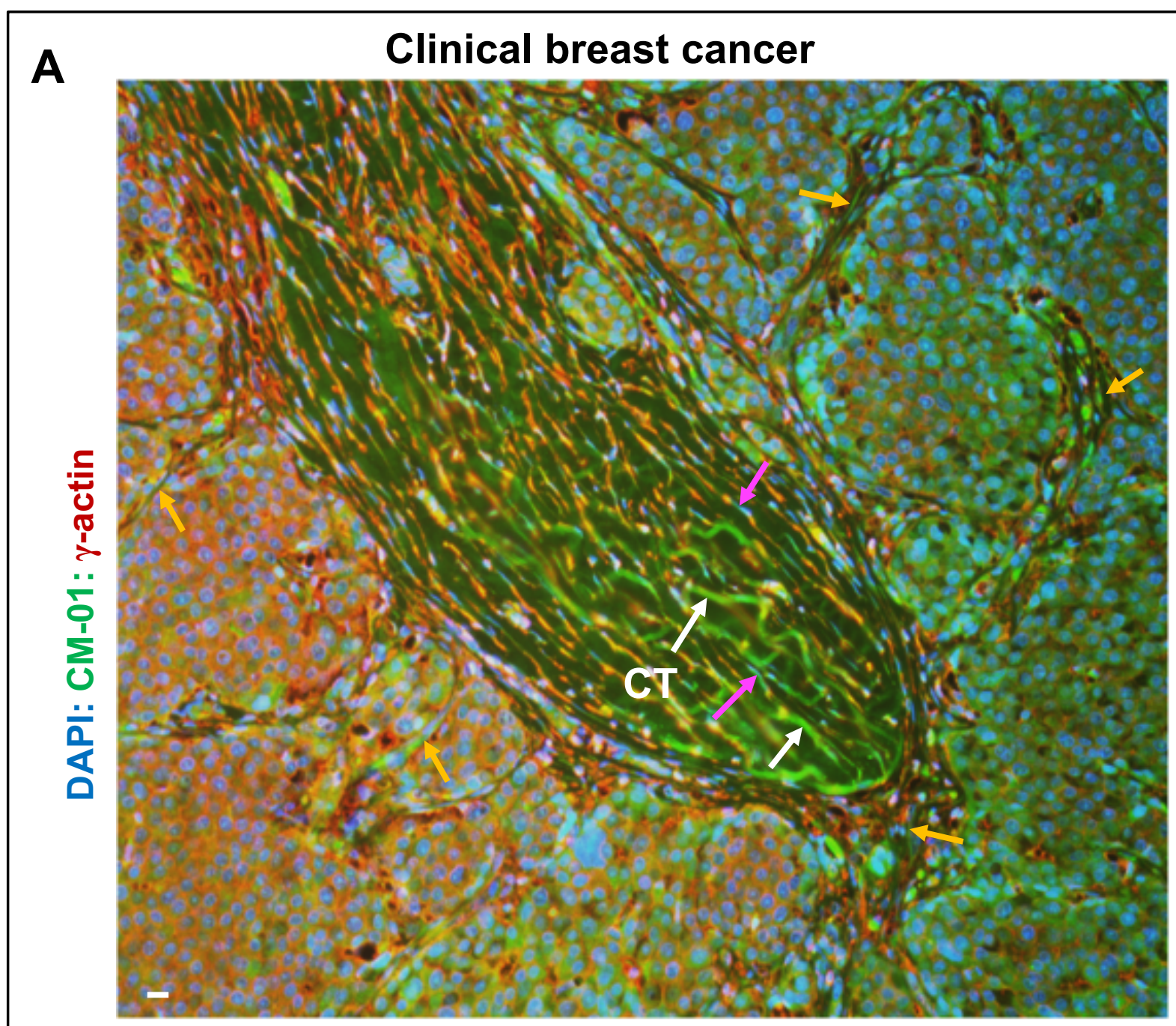

B

\section{Clinical breast cancer}

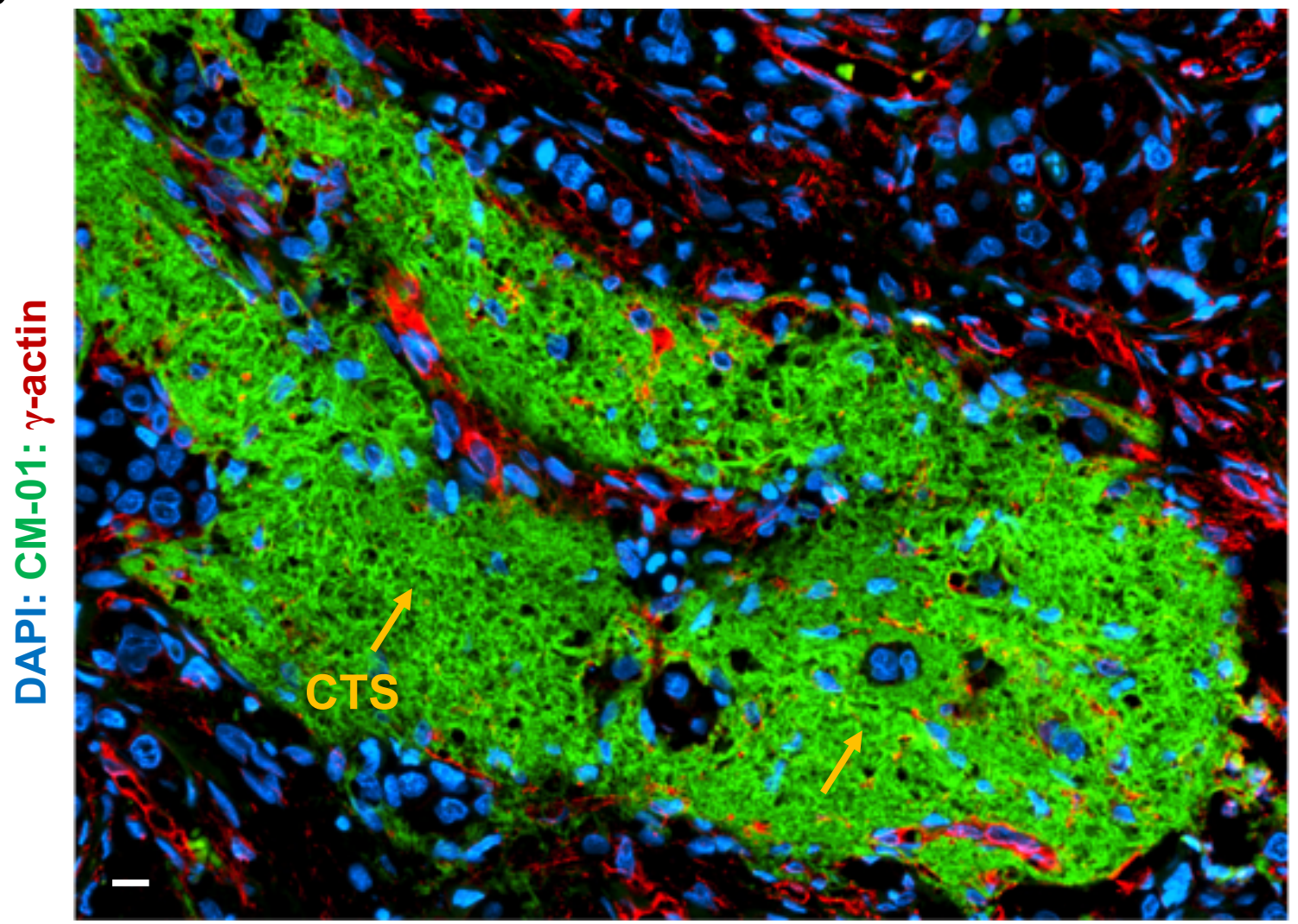




\section{Fig.S19}

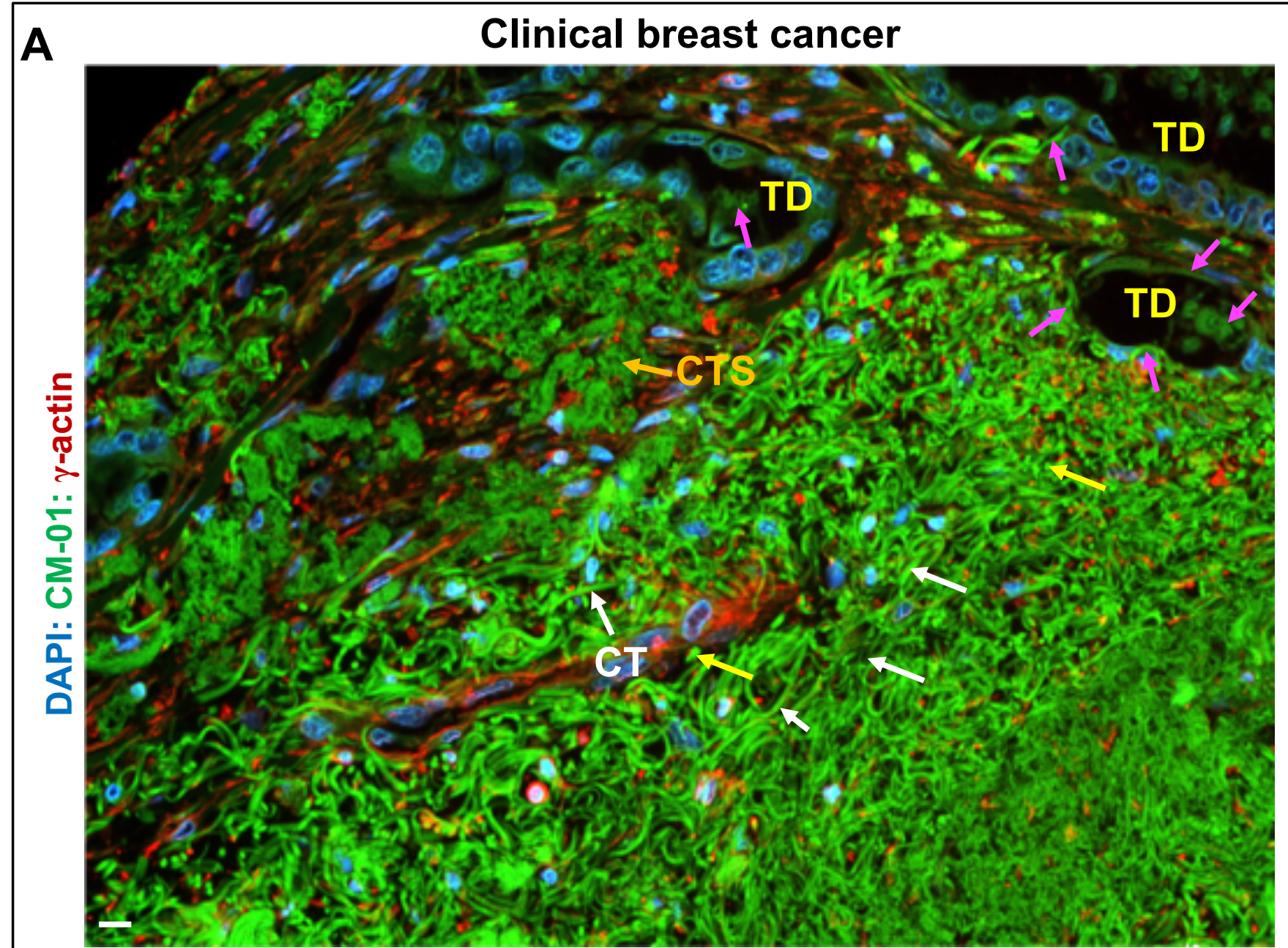

B

Clinical breast cancer

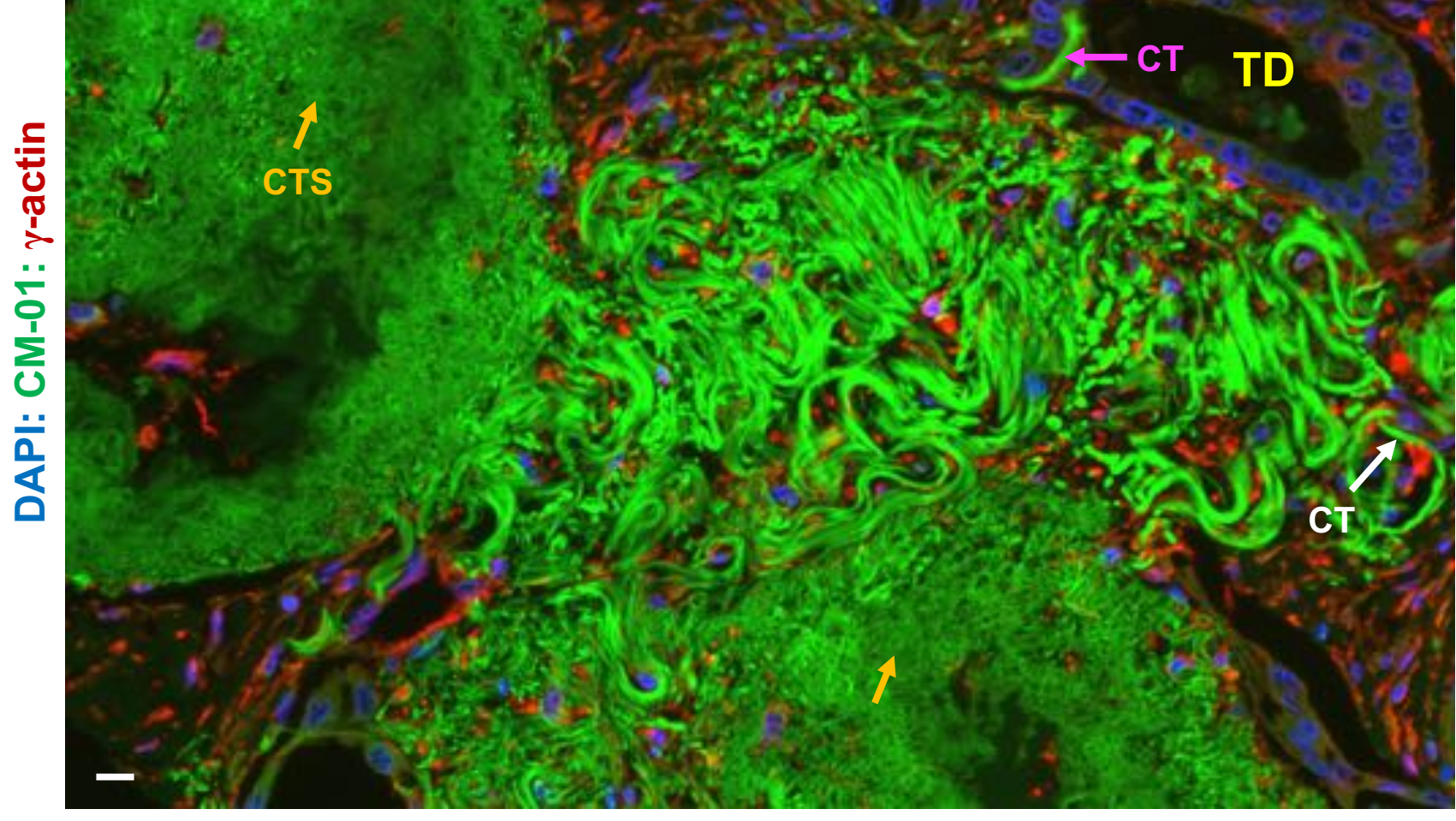




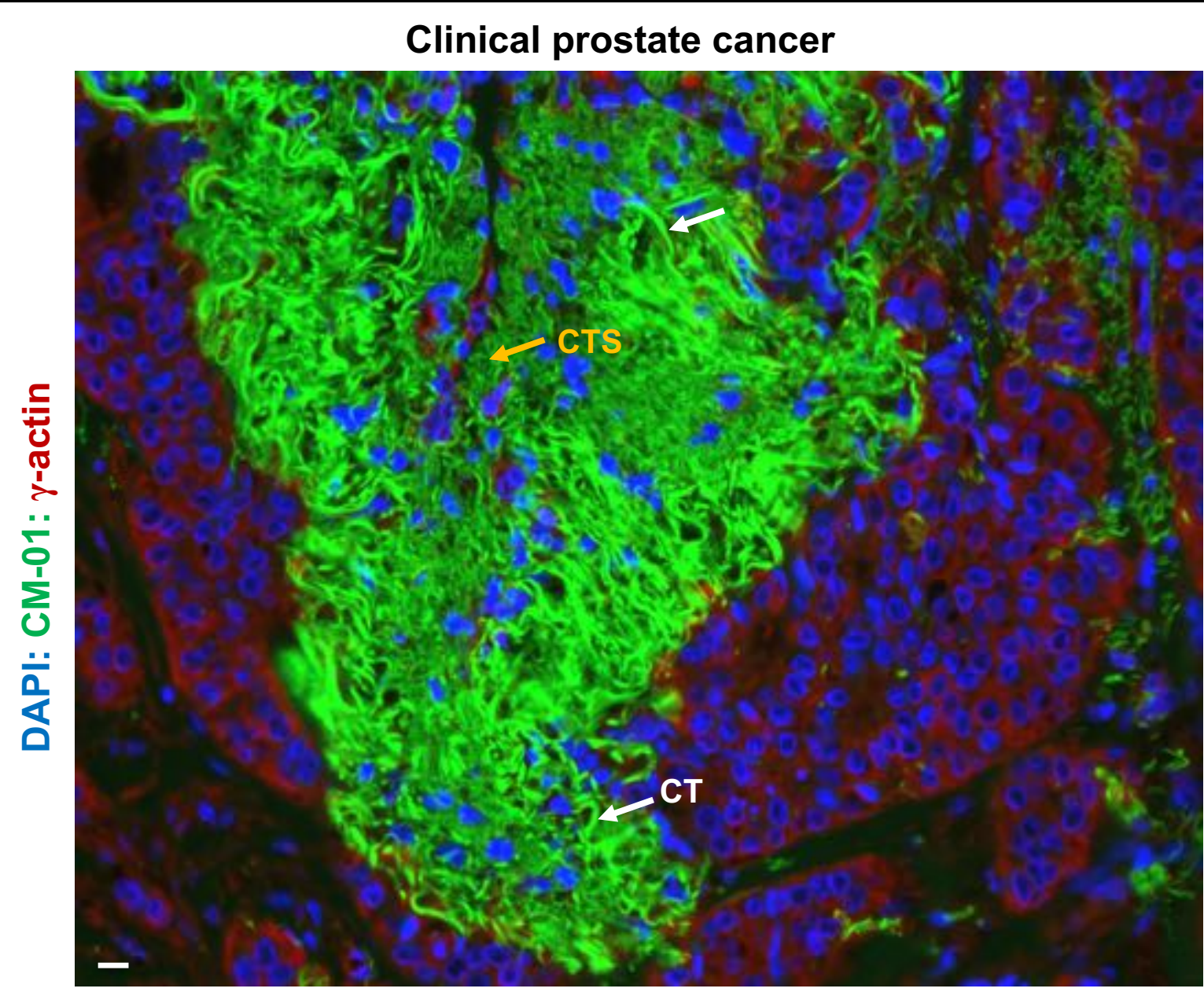

B

Clinical breast cancer

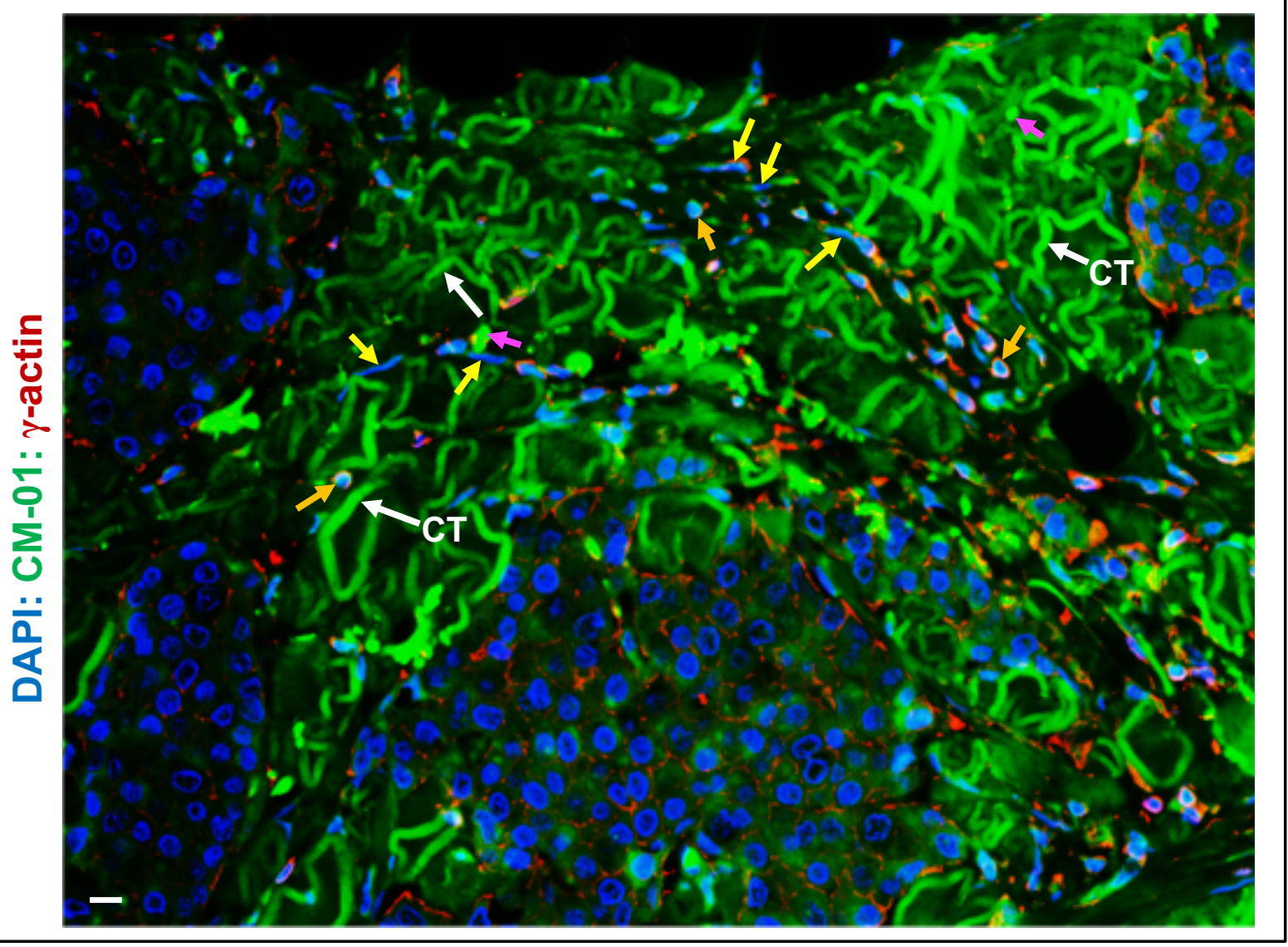




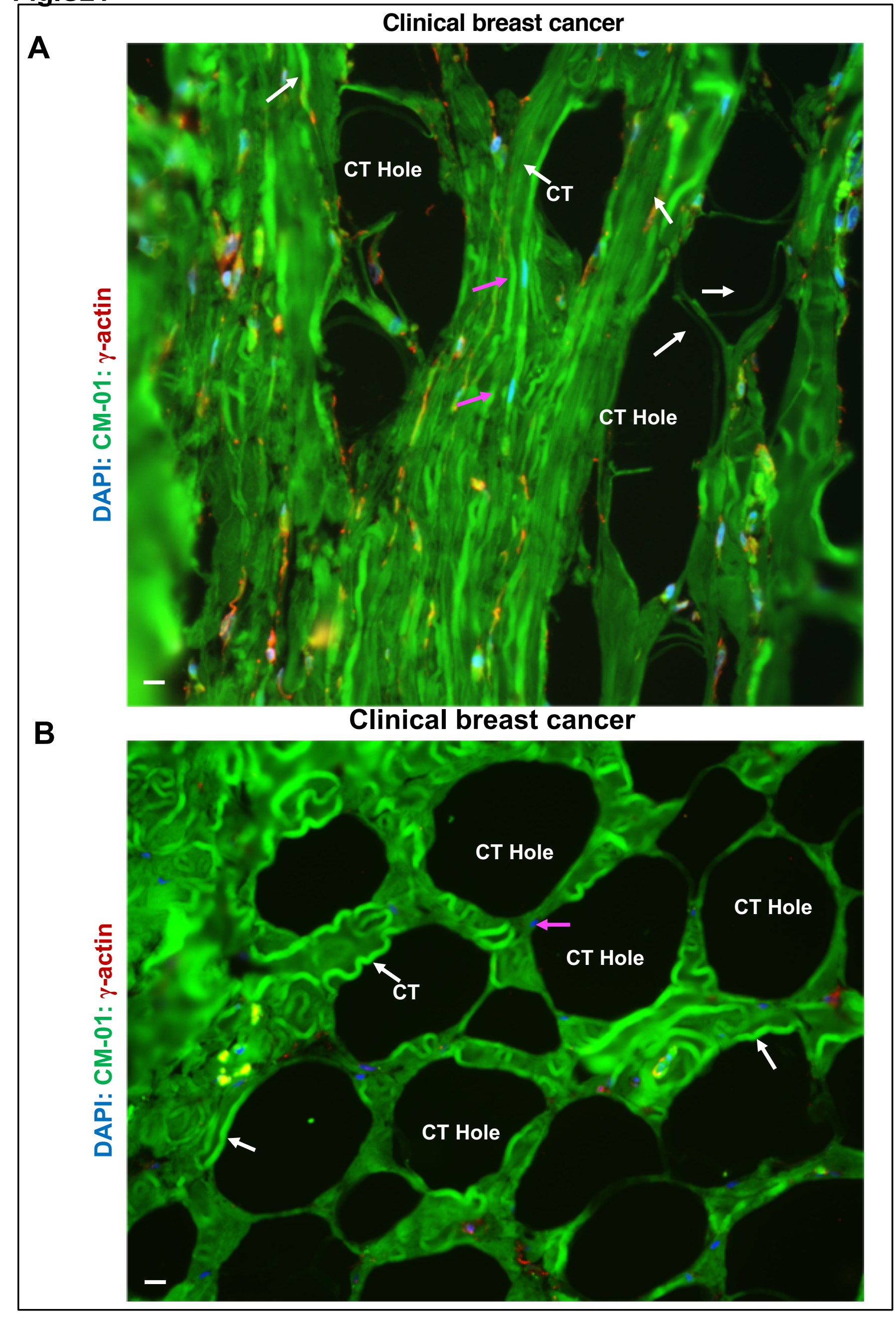




\section{Fig.S22}

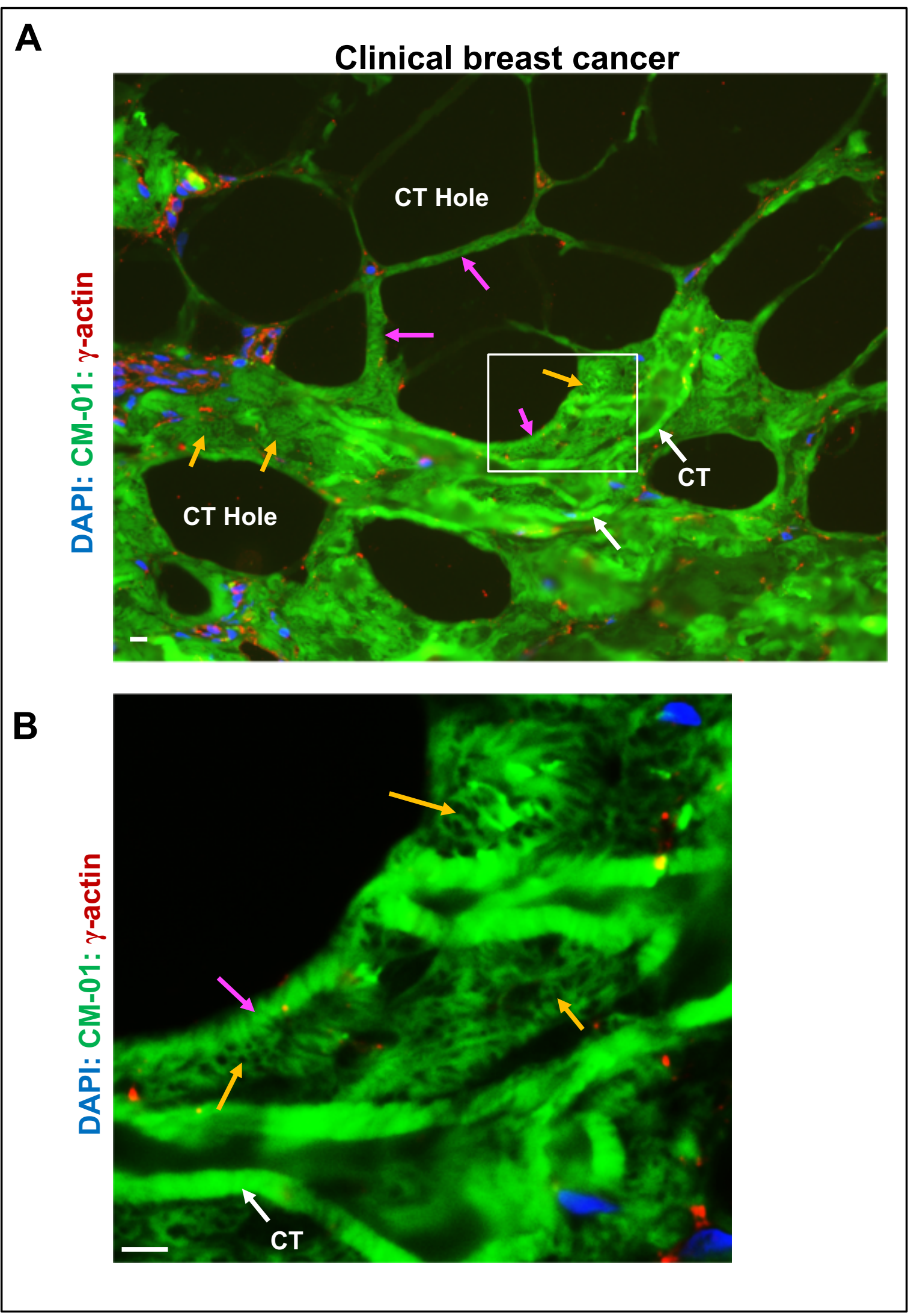




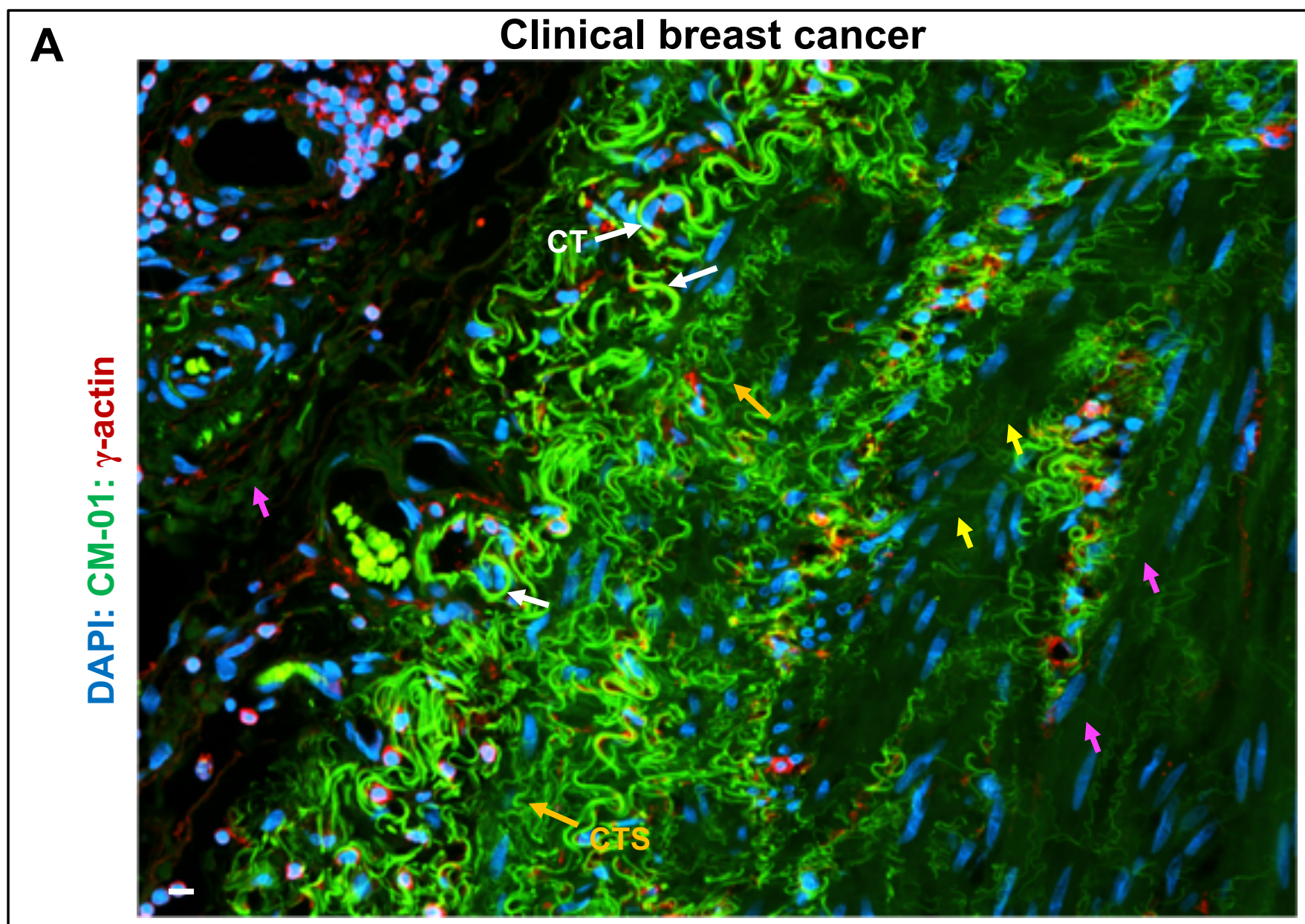

B

\section{Clinical colon cancer}

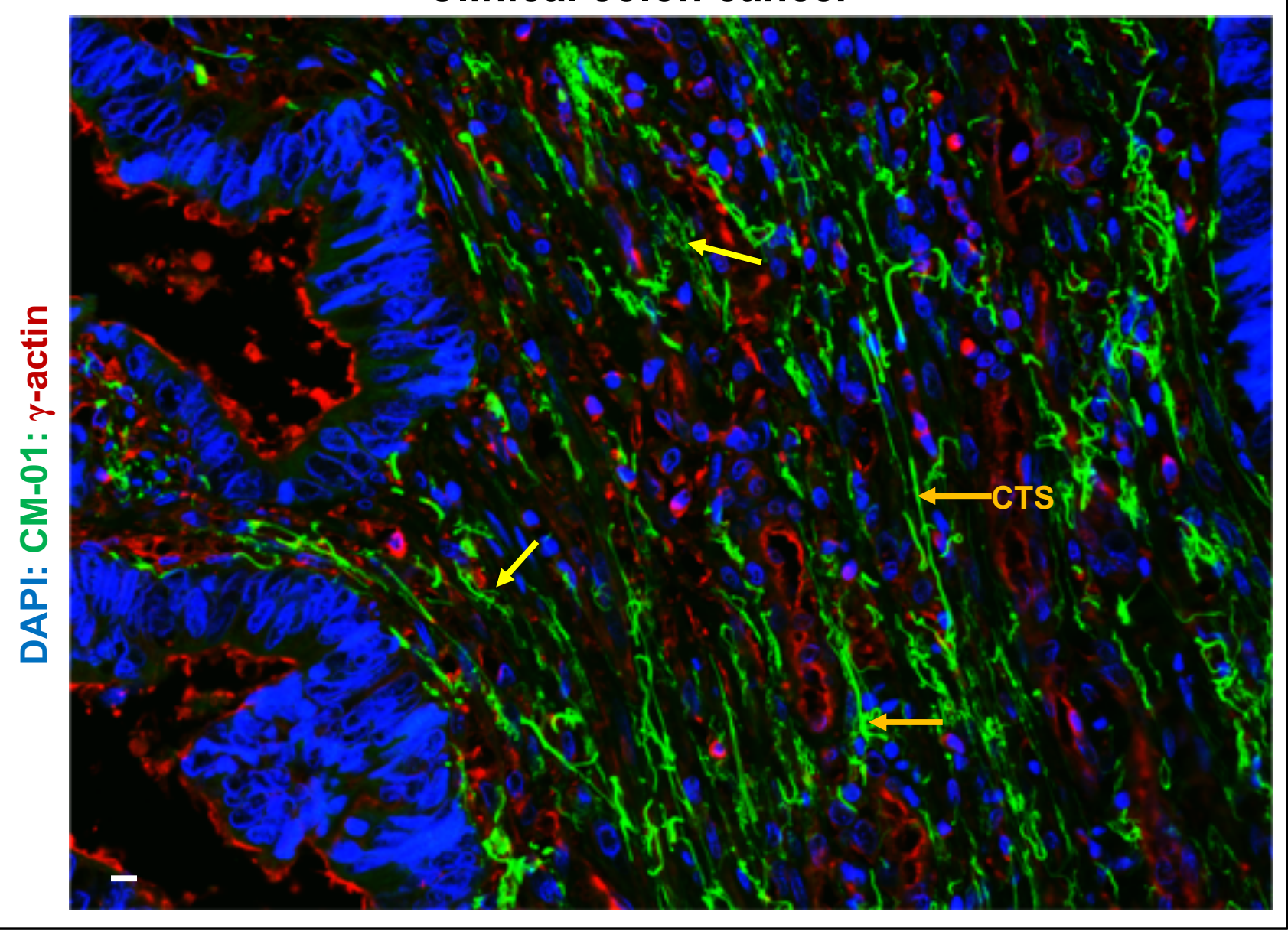




\section{Fig.S24}

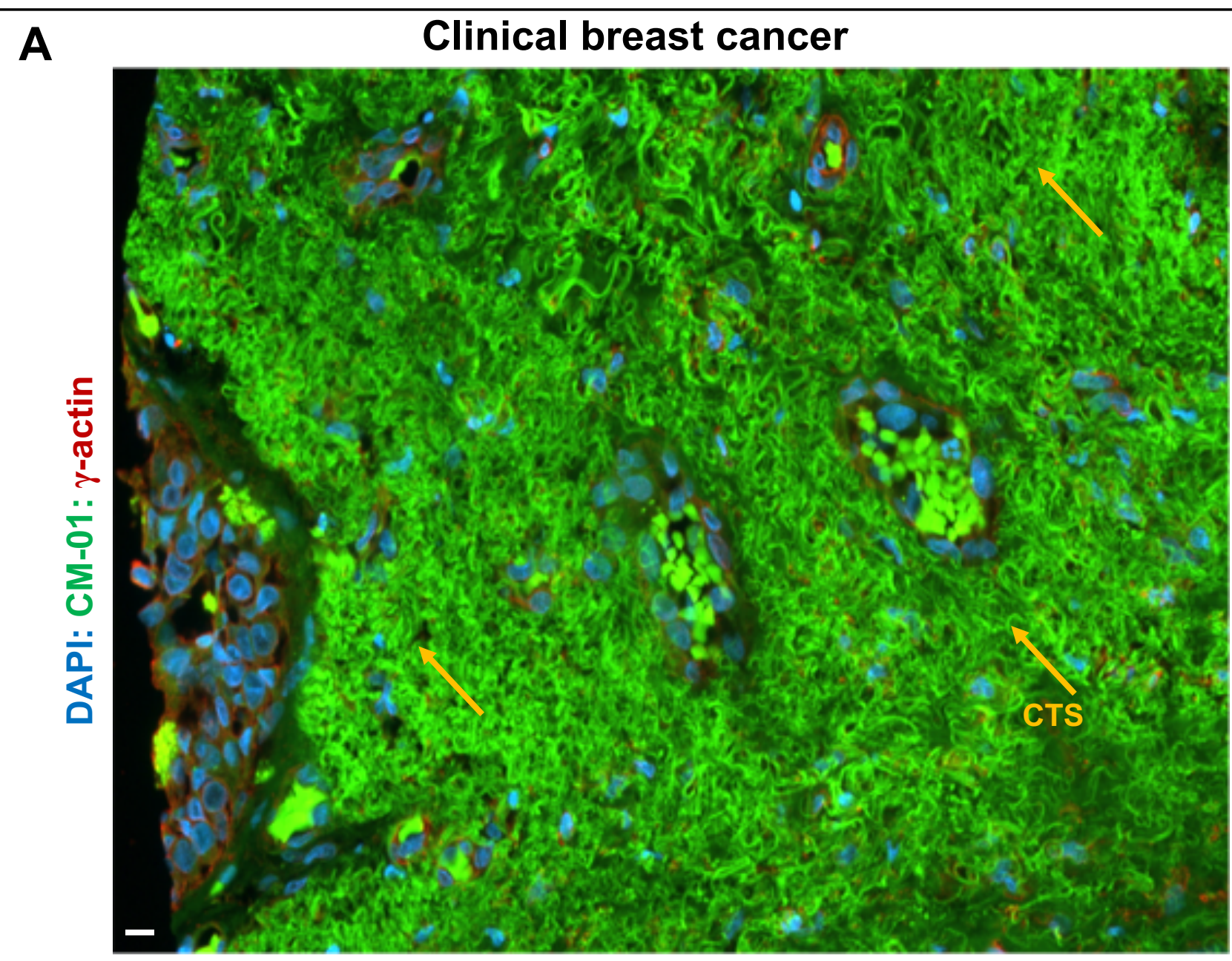

B

\section{Clinical breast cancer}

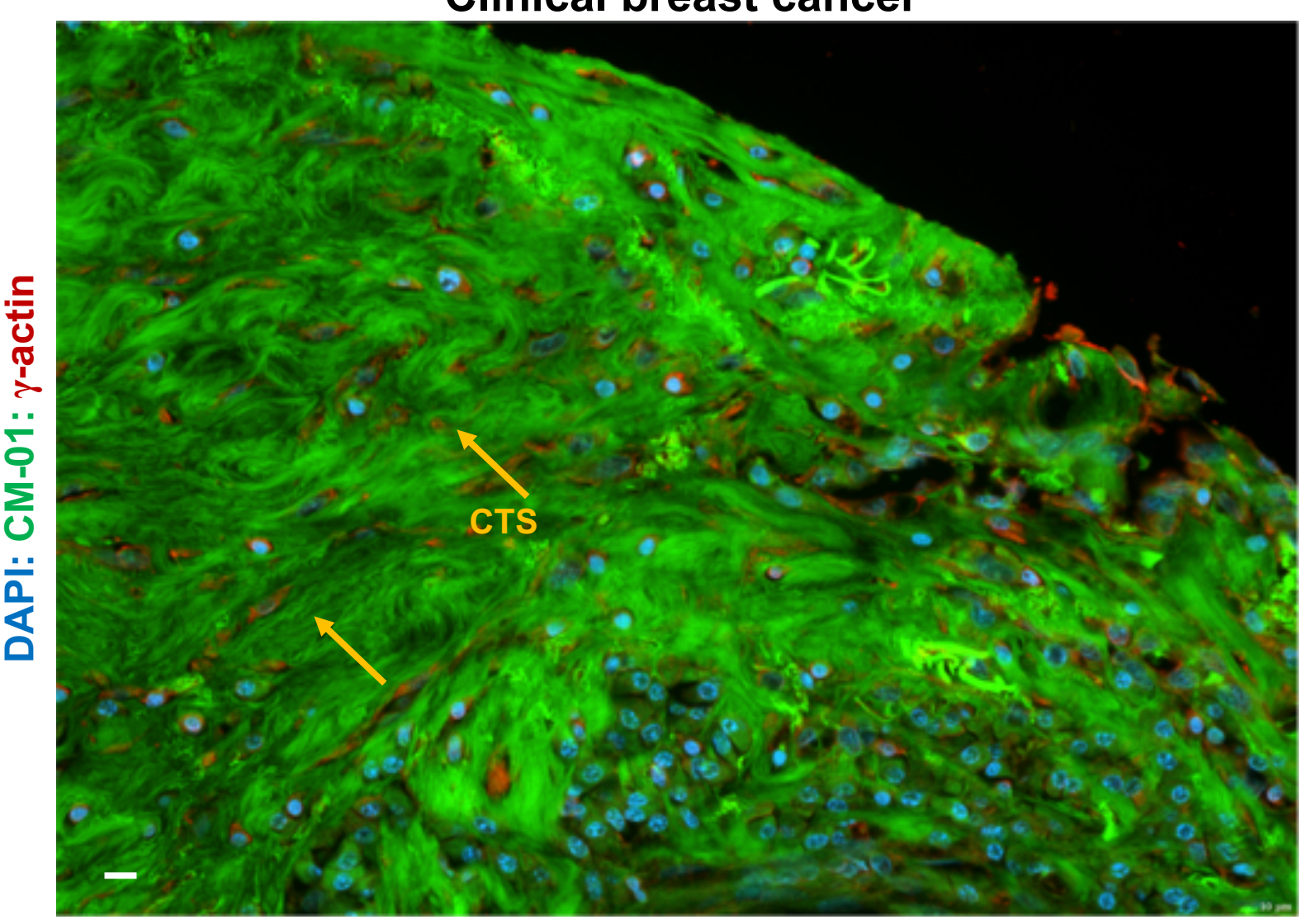




\section{Fig. S25}

A

Clinical prostate cancer

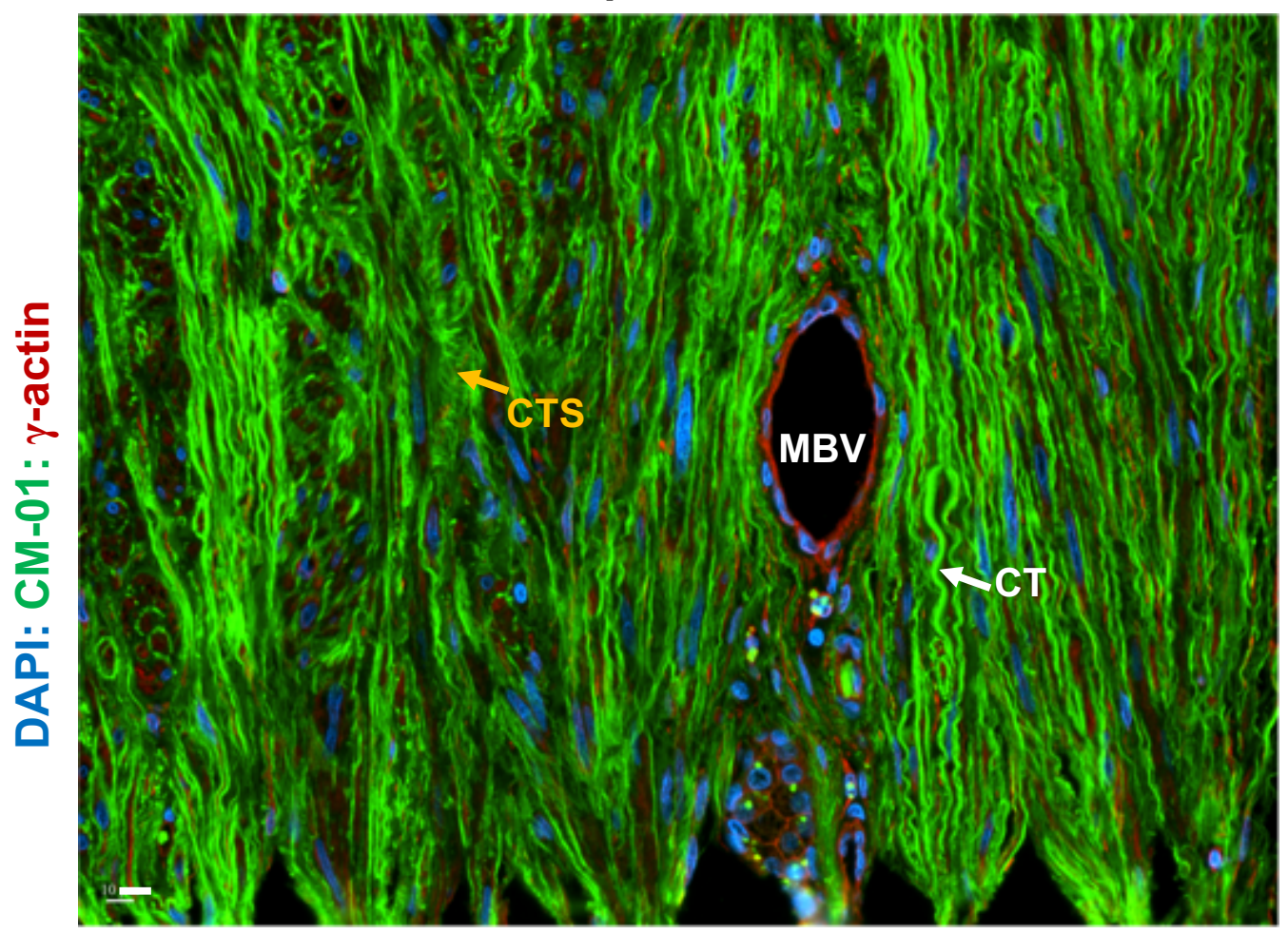

B

Comparison of cytocapsular tubes and humoral vessels in cancer, paracancer tissues and metastatic cancer.

Average ratios of numbers of cytocapsular tubes to numbers of blood vessels and lymph vessels

\begin{tabular}{lccc}
\hline Cancer types & Original cancer niche & Paracancer & metastatic cancer \\
\hline Breast & $1003 \pm 12$ & $1215 \pm 25$ & $1106 \pm 17$ \\
Colon & $987 \pm 11$ & $1112 \pm 23$ & $1056 \pm 18$ \\
Lung & $997 \pm 21$ & $1107 \pm 14$ & $1067 \pm 23$ \\
Liver & $1005 \pm 18$ & $1108 \pm 22$ & $991 \pm 16$ \\
Ovary & $1104 \pm 23$ & $1108 \pm 17$ & $1106 \pm 15$ \\
Pancreas & $1109 \pm 25$ & $1116 \pm 14$ & $1009 \pm 24$ \\
Prostate & $993 \pm 17$ & $1008 \pm 22$ & $997 \pm 26$ \\
Stomach & $1013 \pm 18$ & $1122 \pm 27$ & $1008 \pm 16$ \\
\hline
\end{tabular}




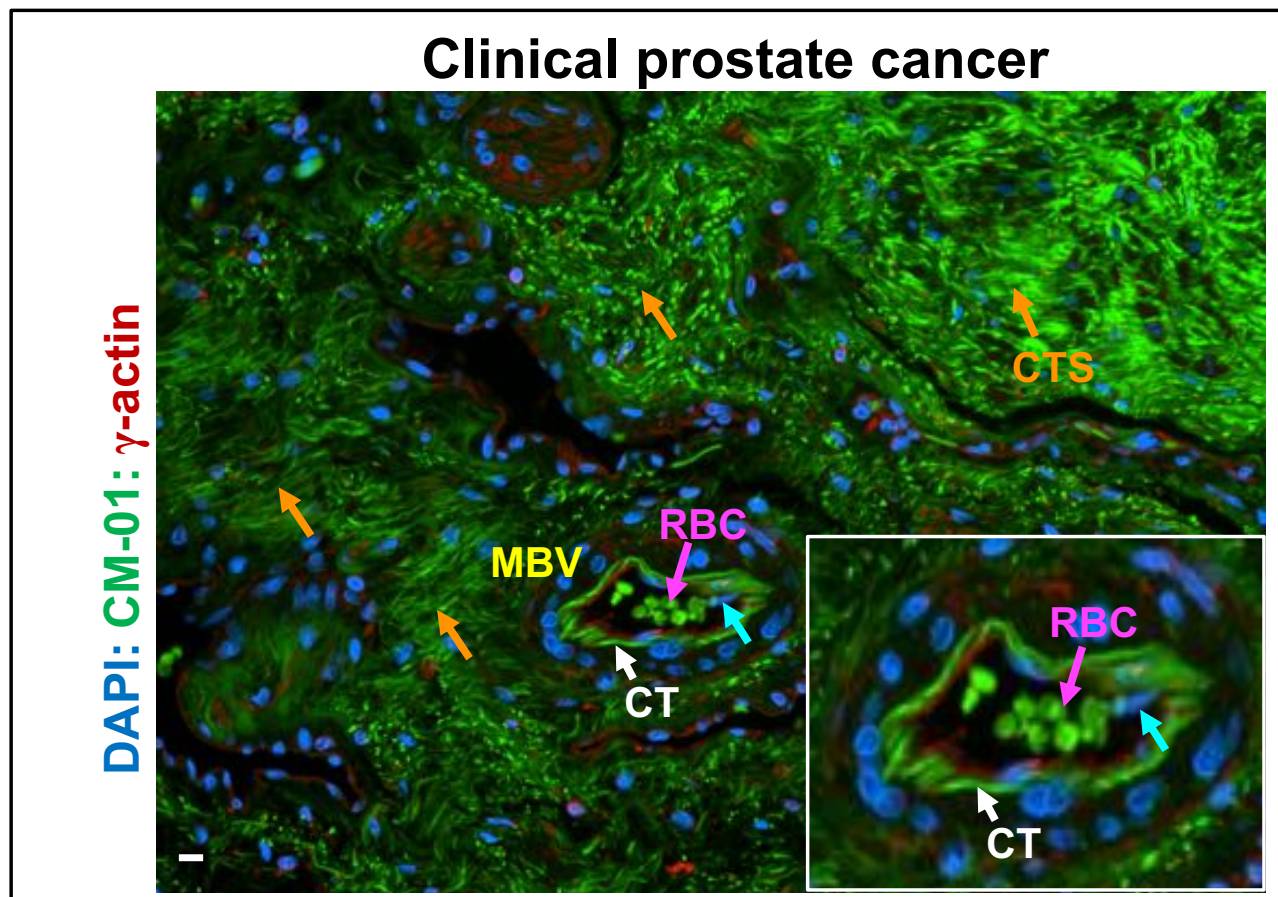

\section{Clinical lung cancer}

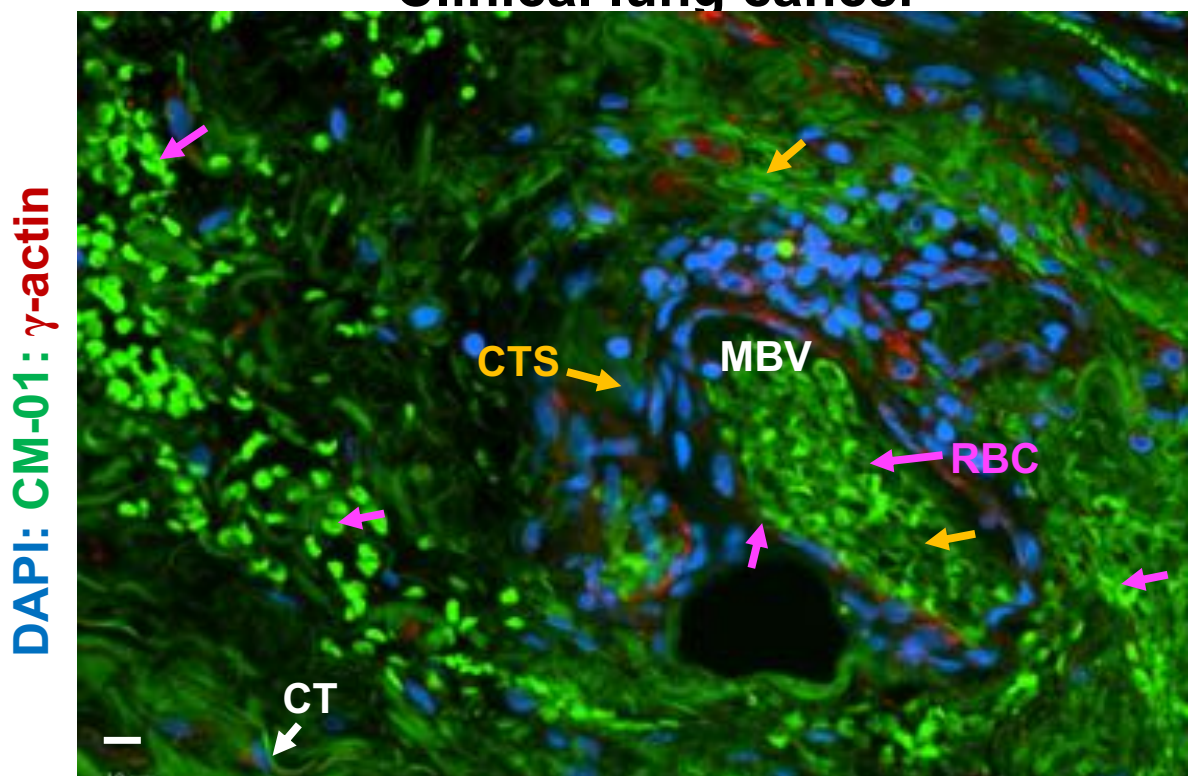

Clinical prostate cancer

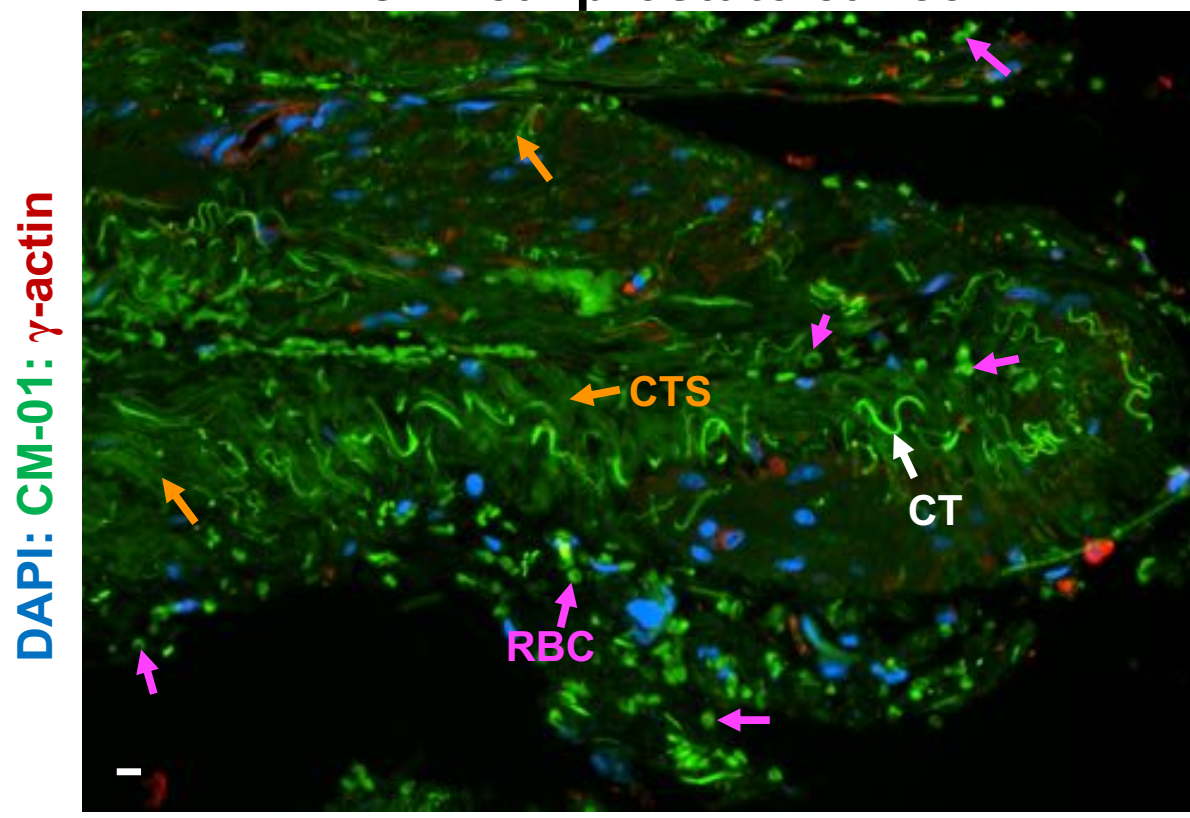




\section{Fig.S27}

\section{Needle biopsy of suspicious breast tumor}

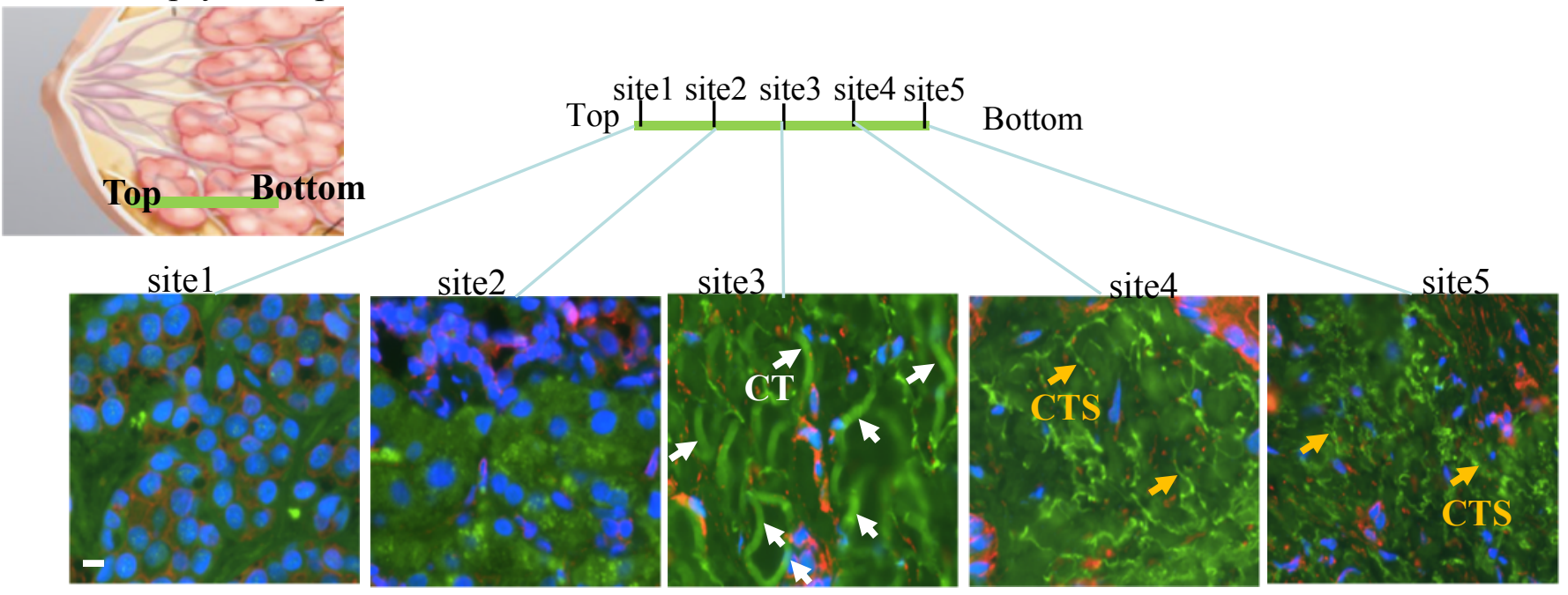

DAPI: CM-01: $\gamma$-actin 
Table S1. Clinical characteristics of cytocapsular tubes (CTs) in benign tumor tissues.

\begin{tabular}{|c|c|c|c|c|}
\hline Benign tumor types & Subtypes & Patients/specimens & with $\mathrm{CT}$ & $\%$ with CT \\
\hline \multirow{11}{*}{ Breast } & Fibrosis and Simple Cysts & 11 & & \\
\hline & Ductal or Lobular Hyperplasia & 13 & 3 & \\
\hline & Lobular Carcinoma in Situ & 2 & & \\
\hline & Adenosi & 1 & & \\
\hline & Fibroadenomas & 1 & & \\
\hline & Phyllodes Tumors & 1 & 1 & \\
\hline & Intraductal Papillomas & 8 & 1 & \\
\hline & Granular Cell Tumors & 3 & 1 & \\
\hline & Fat Necrosis and Oil Cysts & 1 & & \\
\hline & Mastitis & 2 & & \\
\hline & Duct Ectasia & 2 & & \\
\hline \multirow{3}{*}{ Lung } & Hamartomas & 2 & & \\
\hline & Bronchial adenomas & 3 & & \\
\hline & Papillomas & 3 & & \\
\hline Prostate & Benign prostatic hyperplasia & 4 & 1 & \\
\hline \multirow{10}{*}{ Stomach } & fundic gland polyp & 12 & 4 & \\
\hline & hyperplastic polyp & 1 & & \\
\hline & inflammatory fibroid polyps & 2 & & \\
\hline & xanthoma & 1 & & \\
\hline & hamartomatous polyps & 1 & & \\
\hline & leiomyoma & 1 & & \\
\hline & lipoma & 1 & & \\
\hline & hemangioma & 2 & & \\
\hline & glomus tumour & 2 & 1 & \\
\hline & cystic tumour & 1 & & \\
\hline \multirow{3}{*}{ Liver } & Hemangioma & 1 & & \\
\hline & Focal nodular hyperplasia & 1 & & \\
\hline & Hepatocellular adenoma & 2 & & \\
\hline \multirow[b]{9}{*}{ Colon } & Hyperplastic polyps & 15 & 3 & \\
\hline & Hamartomas & 11 & 1 & \\
\hline & Juvenile polyps & 4 & & \\
\hline & Inflammatory polyps & 2 & 1 & \\
\hline & Lymphoid polyps & 4 & & \\
\hline & Tubular adenomas & 1 & & \\
\hline & Villous adenomas & 2 & & \\
\hline & Tubulovillous adenomas & 2 & & \\
\hline & & 126 & 17 & $13.40 \%$ \\
\hline
\end{tabular}


Table S2. Subtypes of cancers with 10 (or more) kinds of investigated subtypes in Table 2.

\begin{tabular}{|c|c|c|c|c|}
\hline $\begin{array}{l}\text { Cancer } \\
\text { types }\end{array}$ & & Cancer subtypes & $\begin{array}{l}\text { Cancer } \\
\text { types }\end{array}$ & Cancer subtypes \\
\hline & 1 & Chondroblastic osteosarcoma of left thigh & & 1 Adenocarcinoma \\
\hline & 2 & Chondrosarcoma of lower limb & & 2 Adenosquamous carcinoma \\
\hline & 3 & Fibroblastic osteosarcoma of right lower femur & & 3 Atypical carcinoid \\
\hline & 4 & Giant cell tumor of bone & & 4 Basal cell carcinoma \\
\hline & 5 & Giant cell tumor of left femur & & 5 Bronchioloalveolar carcinoma \\
\hline & & & & Complexed small cell carcinoma and \\
\hline Bone & 6 & Osteosarcoma of left knee (sparse) & & 6 adenocarcinoma \\
\hline & 7 & Osteosarcoma of left tibia & & 7 Giant cell carcinoma \\
\hline & 8 & Osteosarcoma of right scapula & & 8 Invasive adenocarcinoma \\
\hline & 9 & Osteoblastic osteosarcoma of left upper tibia & & 9 Large cell carcinoma \\
\hline & 10 & Osteosarcoma of right upper femur & & 10 Large cell neuroendocrine carcinoma \\
\hline & 11 & Osteoblastic osteosarcoma of left lower femur & & 11 Large cell undifferentiated carcinoma \\
\hline & & Undiferentiated chondrosarcoma of pars sacralis & & 12 Mucinous adenocarcinoma \\
\hline & 1 & Acinic cell carcinoma & & 13 Mucinous bronchioloalveolar carcinoma \\
\hline & 2 & Adenoid cystic carcinoma & Lung & 14 Non-small cell carcinoma \\
\hline & & Bilateral breast carcinoma and non-synchronous breast & & Non-small cell carcinoma Squamous cell \\
\hline & 3 & & & $\begin{array}{l}15 \text { carcinoma } \\
\text { Non-small cell Invasive papillary }\end{array}$ \\
\hline & 4 & Carcinoid & & 16 adenocarcinoma \\
\hline & 5 & Carcinoma with apocrine differentiation & & 17 Non-small cell lung adenocarcinoma \\
\hline & 6 & Carcinoma with medullary features & & 18 Papillary adenocarcinoma \\
\hline & 7 & Carcinoma with neuroendocrine features & & 19 Pleomorphic carcinoma \\
\hline & 8 & Exceptionally rare types and variants & & 20 Sarcomatoid carcinoma \\
\hline Breast & 9 & Glycogen-rich clear cell carcinoma & & 21 Small cell carcinoma \\
\hline & & Inflammatory carcinoma & & 22 Solid adenocarcinoma \\
\hline & & Infiltrating ductal carcinoma, & & 23 Squamous cell carcinoma \\
\hline & & Invasive carcinoma of no special type & & 1 Acinar cell carcinoma \\
\hline & 13 & Invasive lobular carcinoma & & 2 Adenocarcinoma \\
\hline & 14 & Invasive micropapillary carcinoma & & 3 Adenosquamous carcinoma \\
\hline & 15 & Invasive papillary adenocarcinoma & & 4 Carcinoid \\
\hline & 16 & Invasive papillary carcinoma & & 5 Cystic-solid pseudopapillary carcinoma \\
\hline & 17 & Lipid-rich carcinoma & & 6 Duct adenocarcinoma \\
\hline & 18 & Medullary carcinoma & Pancreas & 7 Islet cell carcinoma \\
\hline & & & & Low-grade malignant cystic - solid \\
\hline & 19 & Metaplastic carcinoma & & 8 papillary tumor \\
\hline & 20 & Mixed invasive lobular and ductal carcinoma & & 9 Mucous adenocarcinoma \\
\hline & & Mucinous adenocarcinoma & & 10 Neuroendocrine tumors of the pancreas \\
\hline & & Mucinous carcinoma and carcinoma with & & \\
\hline & 22 & Mucoepidermoid carcinoma & & 11 Papillary adenocarcinoma \\
\hline & 23 & Neuroendocrine carcinoma & & 12 Squamous cell carcinoma \\
\hline & 24 & Oncocytic carcinoma & & 13 Undifferentiated carcinoma \\
\hline & 25 & Polymorphous carcinoma & & \\
\hline & 26 & Salivary gland/skin adnexal type tumours & & \\
\hline & 27 & Sebaceous carcinoma & & \\
\hline & 1 & Carcinoma sarcomatodes & & \\
\hline & 2 & Chromophobe carcinoma & & \\
\hline & 3 & Clear cell carcinoma & & \\
\hline & 4 & Collecting duct carcinoma & & \\
\hline & 5 & Granular cell carcinoma & & \\
\hline Kidney & 6 & High grade urothelial carcinoma & & \\
\hline & & Low grade urothelial carcinoma & & \\
\hline & 8 & Nephroblastoma & & \\
\hline & 9 & Papillary renal cell carcinoma & & \\
\hline & 10 & Transitional cell carcinoma & & \\
\hline
\end{tabular}


Table S3: Cytocapsular tube (CT) density and suggested cytocapsular tube-based cancer metastasis $(\mathrm{CM})$ grades:

No CT

CM grade 1 (CT: 1-10/mm², with no CT degradation )

CM grade 2 (CT: $11-40 / \mathrm{mm}^{2}$, with no CT degradation)

CM grade 3 (CT: 41-80/mm², with/without a few CT degradation)

CM grade 4 (CT: $>80 / \mathrm{mm}^{2}$, or with many and severe CT degradation)

Table S4: Relationship between conventional cancer stages and cancer metastasis (CM) grades based on the results in involved in Table S1 and Table 2:

\begin{tabular}{|c|c|}
\hline Cancer stage & Cancer Metastasis (CM) grade \\
\hline 0 & $0,1,2,3,4$ \\
\hline I & $1,2,3,4$ \\
\hline II & $2,3,4$ \\
\hline III & 3,4 \\
\hline IV & 4 \\
\hline
\end{tabular}




\section{Movie.S1}

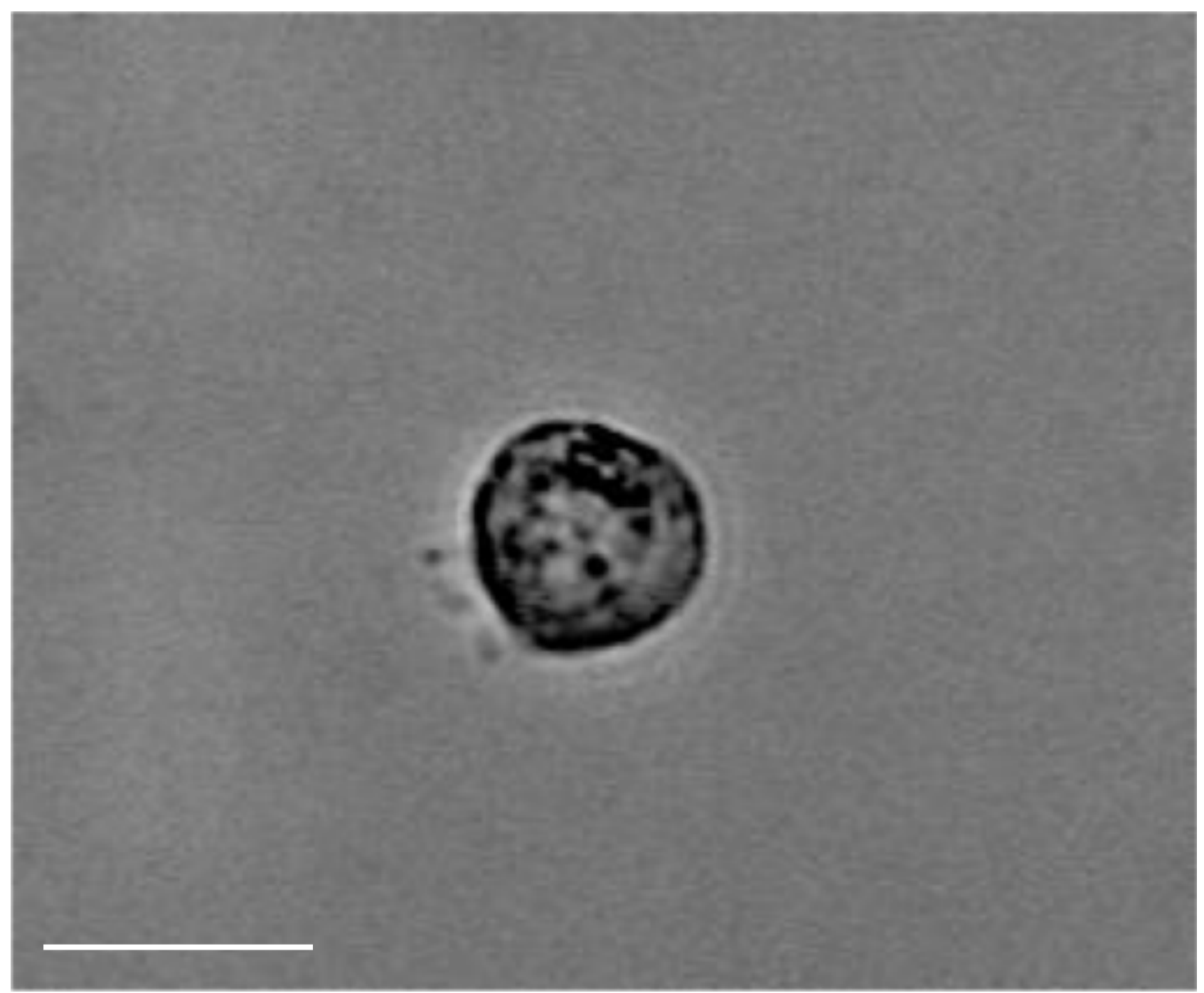




\section{Movie.S2}

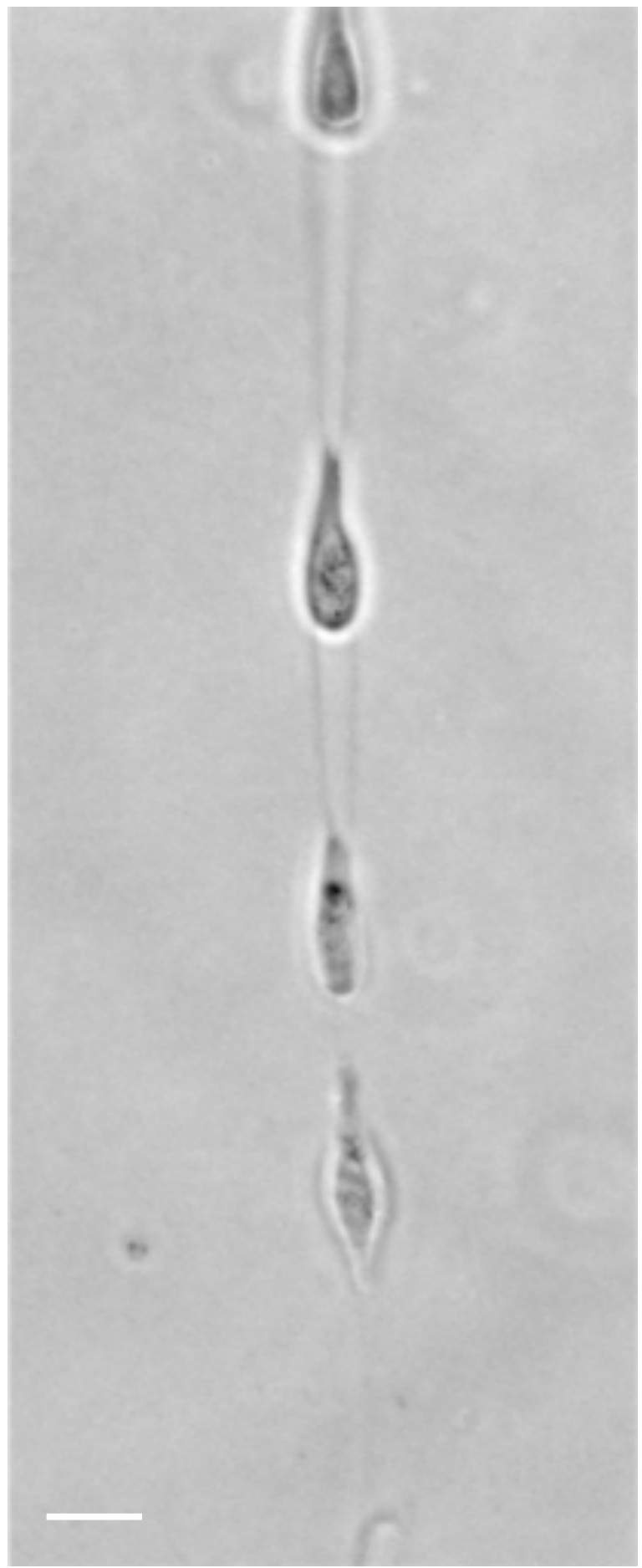




\begin{tabular}{|c|c|c|c|c|c|c|c|c|c|c|c|c|c|c|c|}
\hline No. & Age & Sex & $\begin{array}{l}\text { Organ/ } \\
\text { Anatomic } \\
\text { Site }\end{array}$ & Pathology diagnosis & TNM & Grade & Stage & Tyре & ER & PR & HER2 & $\begin{array}{l}\text { Cytocapsular } \\
\text { tubes (CT) }\end{array}$ & $\begin{array}{c}\mathrm{CT} / \\
\mathrm{mm} \\
2\end{array}$ & $\begin{array}{c}\text { CT } \\
\text { degra } \\
\text { dation }\end{array}$ & $\begin{array}{c}\text { Cancer } \\
\text { metastasis } \\
\text { grade }\end{array}$ \\
\hline 1 & 48 & $\mathrm{~F}$ & Breast & Invasive ductal carcinoma & T1N0M0 & 1 & $\mathrm{I}$ & malignant & + & - & - & + & 6 & - & 1 \\
\hline 2 & 64 & $\mathrm{~F}$ & Breast & Invasive ductal carcinoma & T1N0M0 & 1 & I & malignant & +++ & - & - & + & 9 & - & 1 \\
\hline 3 & 64 & $\mathrm{~F}$ & Breast & Invasive ductal carcinoma & T1N0M0 & 1 & I & malignant & +++ & - & - & + & 6 & - & 1 \\
\hline 4 & 50 & $\mathrm{~F}$ & Breast & Invasive ductal carcinoma & T1N0M0 & 1 & I & malignant & - & * & $1+$ & + & 8 & - & 1 \\
\hline 5 & 61 & $\mathrm{~F}$ & Breast & Invasive ductal carcinoma & T1N0M0 & 1 & I & malignant & ++ & + & - & + & 24 & - & 2 \\
\hline 6 & 61 & $\mathrm{~F}$ & Breast & Invasive ductal carcinoma & T1N0M0 & 1 & I & malignant & ++ & + & - & + & 18 & - & 2 \\
\hline 7 & 48 & $\mathrm{~F}$ & Breast & Invasive ductal carcinoma & T1N0M0 & 1 & I & malignant & + & - & - & + & 12 & - & 2 \\
\hline 8 & 50 & $\mathrm{~F}$ & Breast & Invasive ductal carcinoma & T1N0M0 & 1 & I & malignant & - & * & $1+$ & + & 13 & - & 2 \\
\hline 9 & 40 & $\mathrm{~F}$ & Breast & Invasive ductal carcinoma & T1N0M0 & 1 & I & malignant & + & * & 0 & + & 16 & - & 2 \\
\hline 10 & 40 & $\mathrm{~F}$ & Breast & Invasive ductal carcinoma & T1N0M0 & 1 & I & malignant & + & * & 0 & + & 22 & - & 2 \\
\hline 11 & 56 & $\mathrm{~F}$ & Breast & Invasive ductal carcinoma & T1N0M0 & 1 & I & malignant & +++ & * & 0 & + & 27 & - & 2 \\
\hline 12 & 56 & $\mathrm{~F}$ & Breast & Invasive ductal carcinoma & T1N0M0 & 1 & I & malignant & +++ & * & 0 & + & 11 & - & 2 \\
\hline 13 & 33 & $\mathrm{~F}$ & Breast & Invasive ductal carcinoma & T1N0M0 & 1 & I & malignant & +++ & $*$ & 0 & + & 38 & - & 2 \\
\hline 14 & 67 & $\mathrm{~F}$ & Breast & Invasive ductal carcinoma & T1N0M0 & 1 & I & malignant & ++ & ++ & 0 & + & 38 & - & 2 \\
\hline 15 & 33 & $\mathrm{~F}$ & Breast & Invasive ductal carcinoma & T1N0M0 & 1 & 1 & malignant & +++ & * & 0 & + & 46 & + & 3 \\
\hline 16 & 48 & $\mathrm{~F}$ & Breast & Invasive ductal carcinoma & T1N0M0 & 1 & 1 & malignant & + & - & 0 & + & 51 & - & 3 \\
\hline 17 & 48 & $\mathrm{~F}$ & Breast & Invasive ductal carcinoma & T1N0M0 & 1 & I & malignant & + & * & 0 & + & 42 & + & 3 \\
\hline 18 & 55 & $\mathrm{~F}$ & Breast & Invasive ductal carcinoma & T1N0M0 & 2 & 1 & malignant & - & - & - & + & 45 & - & 3 \\
\hline 19 & 55 & $\mathrm{~F}$ & Breast & Invasive ductal carcinoma & T1N0M0 & 2 & I & malignant & - & - & - & + & 34 & - & 2 \\
\hline 20 & 69 & $\mathrm{~F}$ & Breast & Invasive ductal carcinoma & T1N0M0 & 2 & 1 & malignant & +++ & +++ & - & + & 56 & + & 3 \\
\hline 21 & 63 & $\mathrm{~F}$ & Breast & Invasive ductal carcinoma & T1N0M0 & 2 & 1 & malignant & + & - & - & + & 47 & + & 3 \\
\hline 22 & 63 & $\mathrm{~F}$ & Breast & Invasive ductal carcinoma & T1N0M0 & 2 & I & malignant & + & - & - & + & 41 & - & 3 \\
\hline 23 & 48 & $\mathrm{~F}$ & Breast & Mucinous carcinoma & T1N0M0 & 2 & 1 & malignant & ++ & - & - & + & 53 & - & 3 \\
\hline 24 & 48 & $\mathrm{~F}$ & Breast & Mucinous carcinoma & T1N0M0 & 2 & 1 & malignant & ++ & - & - & + & 58 & + & 3 \\
\hline 25 & 42 & $\mathrm{~F}$ & Breast & Invasive ductal carcinoma & T1N0M0 & 2 & I & malignant & + & - & $2+$ & + & 36 & - & 2 \\
\hline 26 & 42 & $\mathrm{~F}$ & Breast & Invasive ductal carcinoma & T1N0M0 & 2 & I & malignant & + & - & $2+$ & + & 21 & - & 2 \\
\hline 27 & 49 & $\mathrm{~F}$ & Breast & Invasive ductal carcinoma & T1N0M0 & 2 & I & malignant & - & - & - & + & 23 & - & 2 \\
\hline 28 & 49 & $\mathrm{~F}$ & Breast & Invasive ductal carcinoma & T1N0M0 & 2 & I & malignant & - & - & - & + & 32 & - & 2 \\
\hline 29 & 54 & $\mathrm{~F}$ & Breast & Invasive ductal carcinoma & T1N0M0 & 2 & I & malignant & + & + & - & + & 36 & - & 2 \\
\hline 30 & 54 & $\mathrm{~F}$ & Breast & Invasive ductal carcinoma & T1N0M0 & 2 & I & malignant & + & + & - & + & 44 & - & 3 \\
\hline 31 & 54 & $\mathrm{~F}$ & Breast & Invasive ductal carcinoma & T1N0M0 & 2 & I & malignant & ++ & ++ & - & + & 53 & - & 3 \\
\hline 32 & 48 & $\mathrm{~F}$ & Breast & Invasive ductal carcinoma & T1N0M0 & 2 & I & malignant & - & - & - & + & 31 & - & 2 \\
\hline 33 & 48 & $\mathrm{~F}$ & Breast & Invasive ductal carcinoma & T1N0M0 & 2 & I & malignant & - & - & - & + & 24 & - & 2 \\
\hline 34 & 44 & $\mathrm{~F}$ & Breast & Invasive ductal carcinoma & T1CN0M0 & 2 & I & malignant & + & +++ & * & + & 36 & - & 2 \\
\hline 35 & 44 & $\mathrm{~F}$ & Breast & Invasive ductal carcinoma & T1cN0M0 & 2 & I & malignant & * & +++ & 0 & + & 42 & - & 3 \\
\hline
\end{tabular}


Breast

Breast

Breast

Breast

Breast

Breast

Breast

Breast

Breast

Breast

Breast

Breast

Breast

Breast

Breast

Breast

Breast

Breast

Breast

Breast

Breast

Breast

Breast

Breast

Breast

Breast

Breast

Breast

Breast

Breast

Breast

Breast

Breast

Breast

Breast

Breast

Breast

Breast

Breast

Breast
Invasive ductal carcinoma

Invasive ductal carcinoma

Invasive ductal carcinoma

Invasive ductal carcinoma

Invasive ductal carcinoma

Invasive ductal carcinoma

Invasive ductal carcinoma

Invasive ductal carcinoma

Invasive ductal carcinoma

Invasive ductal carcinoma

Mucinous carcinoma

Mucinous carcinoma

Invasive ductal carcinoma

Invasive ductal carcinoma

Invasive ductal carcinoma

Invasive ductal carcinoma

Invasive ductal carcinoma

Invasive ductal carcinoma

Invasive ductal carcinoma

Invasive ductal carcinoma

Invasive ductal carcinoma

Invasive ductal carcinoma

Invasive ductal carcinoma

Invasive ductal carcinoma

Invasive ductal carcinoma

Invasive ductal carcinoma

T1CNOM0 2

T1CNOMO 2

T1CNOMO 2

T1CNOMO 2

T1NOM0 2

T1NOMO 2

T1CNOM0 2

T1CNOMO 2

T1CNOMO 2

T1CNOM0 2

T1CNOMO 2

T1CNOMO 2

T1NOMO 2

T1NOMO 2

T1NOMO 2

T1NOMO 2

T1NOMO 2

T1NOMO 2

T1NOMO 2

T1NOMO 2

T1NOMO 2

T1NOMO 2

T1NOMO 2

T1NOMO 2

T1NOMO 2

T1NOMO 2

nvasive ductal carcinoma (sparse T1NOMO 2

Invasive ductal carcinoma

Invasive ductal carcinoma

T1NOM0

T1N0M0

T1NOMO

T1NOMO

T1NOMO

T1NOMO

T1NOMO

T1NOMO

T1NOMO

carcino ductal

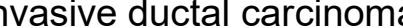

T1NOMO

T1CNOM0

Invasive ductal carcinoma

Invasive ductal carcinoma

T1CNOM0

asive ductal carcinoma with necrc T1N0M0

$\begin{array}{cccc}\text { malignant } & ++ & ++ & 0 \\ \text { malignant } & ++ & ++ & 0 \\ \text { malignant } & - & - & 0 \\ \text { malignant } & - & - & 0 \\ \text { malignant } & ++ & + & 0 \\ \text { malignant } & ++ & + & 0 \\ \text { malignant } & - & - & 0 \\ \text { malignant } & - & - & 0 \\ \text { malignant } & + & + & 0 \\ \text { malignant } & + & + & 0 \\ \text { malignant } & * & * & 0 \\ \text { malignant } & * & * & 0 \\ \text { malignant } & - & - & 0 \\ \text { malignant } & - & - & 0 \\ \text { malignant } & - & - & 0 \\ \text { malignant } & - & - & 0 \\ \text { malignant } & ++ & ++ & 0 \\ \text { malignant } & ++ & ++ & 0 \\ \text { malignant } & + & + & 0 \\ \text { malignant } & + & + & 0 \\ \text { malignant } & + & ++ & 0 \\ \text { malignant } & + & ++ & 0 \\ \text { malignant } & ++ & - & 0 \\ \text { malignant } & ++ & - & 0 \\ \text { malignant } & + & + & 0 \\ \text { malignant } & + & + & 0 \\ \text { malignant } & - & - & 0 \\ \text { malignant } & +++ & - & 0 \\ \text { malignant } & +++ & + & 0 \\ \text { malignant } & - & - & 0 \\ \text { malignant } & ++ & - & 0 \\ \text { malignant } & ++ & - & 0 \\ \text { malignant } & - & - & 0 \\ \text { malignant } & +++ & - & 0 \\ \text { malignant } & +++ & - & 0 \\ \text { malignant } & ++ & ++ & 0 \\ \text { malignant } & ++ & ++ & 0 \\ \text { malignant } & ++ & ++ & 0 \\ \text { malignant } & ++ & ++ & 0 \\ \text { malignant } & +++ & ++ & 0\end{array}$

$\begin{array}{lll}33 & - & 2 \\ 52 & - & 3 \\ 22 & - & 2 \\ 38 & - & 2 \\ 31 & - & 2 \\ 46 & - & 3 \\ 49 & - & 3 \\ 60 & - & 3 \\ 55 & - & 3 \\ 32 & - & 2 \\ 26 & - & 2 \\ 29 & - & 2 \\ 35 & - & 2 \\ 32 & - & 2 \\ 46 & - & 3 \\ 36 & - & 2 \\ 47 & - & 3 \\ 41 & - & 3 \\ 52 & - & 3 \\ 49 & - & 3 \\ 55 & - & 3 \\ 36 & - & 2 \\ 32 & - & 2 \\ 46 & - & 3 \\ 48 & - & 3 \\ 44 & - & 3 \\ 32 & - & 2 \\ 28 & - & 2 \\ 54 & - & 3 \\ 21 & - & 2 \\ 62 & - & 3 \\ 45 & - & 3 \\ 33 & - & 2 \\ 61 & - & 3 \\ 56 & - & 3 \\ 44 & - & 3 \\ 48 & - & 3 \\ 39 & - & 2 \\ 56 & - & 3 \\ 72 & - & 3 \\ & & \end{array}$


Breast asive ductal carcinoma with necrc T1N0M0 2

Breast

Breast

Breast

Breast

Breast

Breast

Breast

Breast

Breast

Breast

Breast

Breast

Breast

Breast

Breast

Breast

Breast

Breast

Breast

Breast

Breast

Breast

Breast

Breast

Breast

Breast

Breast

Breast

Breast

Breast

Breast

Breast

Breast

Breast

Breast

Breast

Breast

Breast

Breast

carcinoma

nvasive ductal carcinoma

Invasive ductal carcinoma

Invasive ductal carcinoma

Invasive ductal carcinoma

Invasive ductal carcinoma

Invasive ductal carcinoma

Invasive ductal carcinoma

Invasive ductal carcinoma

2

T1cN0M0 2

T1CNOMO 2

T1CNOM0 2

T1CNOMO 2

Invasive ductal carcinoma $1 \mathrm{cNOMO}$

Invasive ductal carcinoma

Invasive ductal carcinoma

T1CNOMO

T1CNOMO

Invasive ductal carcinoma

Invasive ductal carcinoma

Invasive ductal carcinoma

Invasive ductal carcinoma

Invasive ductal carcinoma

Invasive ductal carcinoma

Invasive ductal carcinoma

Invasive ductal carcinoma

Invasive ductal carcinoma

T1CNOMO 2

T1CNOMO 2

T1CNOM0 2

T1CNOMO

T1CNOM0 2

T1CNOMO 2

T1CNOMO 2

T1CNOMO 2

Invasive ductal carcinoma

Invasive ductal carcinoma

Invasive ductal carcinoma

Invasive ductal carcinoma

Invasive ductal carcinoma

Invasive ductal carcinoma

Invasive ductal carcinoma

Invasive ductal carcinoma

Invasive ductal carcinoma

Invasive ductal carcinoma

Invasive ductal carcinoma

Invasive ductal carcinoma

Invasive ductal carcinoma

Invasive ductal carcinoma

Invasive ductal carcinoma

Invasive ductal carcinoma
T1NOMO 2

T1NOMO 2

T1NOMO 2

T1NOMO

T1NOMO 2

T1NOMO

T1NOMO 2

T1NOMO 2

T1N0M0

T1NOMO 2

T1N0M0

T1NOMO 2

T1NOMO 2

T1NOMO 2

T1NOMO 2
T1NOMO

$\begin{array}{cccc}\text { malignant } & +++ & ++ & 0 \\ \text { malignant } & +++ & +++ & 0 \\ \text { malignant } & +++ & +++ & 0 \\ \text { malignant } & +++ & + & 0 \\ \text { malignant } & +++ & + & 0 \\ \text { malignant } & +++ & + & 0 \\ \text { malignant } & +++ & + & 0 \\ \text { malignant } & - & + & 0 \\ \text { malignant } & +++ & ++ & 0 \\ \text { malignant } & +++ & ++ & 0 \\ \text { malignant } & - & - & 3+ \\ \text { malignant } & - & - & 3+ \\ \text { malignant } & +++ & ++ & 3+ \\ \text { malignant } & +++ & ++ & 3+ \\ \text { malignant } & +++ & +++ & 0 \\ \text { malignant } & +++ & +++ & 0 \\ \text { malignant } & - & - & 3+ \\ \text { malignant } & - & - & 3+ \\ \text { malignant } & + & + & 2+ \\ \text { malignant } & + & + & 2+ \\ \text { malignant } & +++ & +++ & 0 \\ \text { malignant } & +++ & +++ & 0 \\ \text { malignant } & ++ & +++ & 0 \\ \text { malignant } & ++ & +++ & 0 \\ \text { malignant } & ++ & - & 0 \\ \text { malignant } & ++ & - & 0 \\ \text { malignant } & - & - & 0 \\ \text { malignant } & - & - & 0 \\ \text { malignant } & + & * & 0 \\ \text { malignant } & + & + & 0 \\ \text { malignant } & + & - & 1+ \\ \text { malignant } & + & - & 1+ \\ \text { malignant } & - & * & 0 \\ \text { malignant } & - & * & 0 \\ \text { malignant } & - & - & 2+ \\ \text { malignant } & - & - & 2+ \\ \text { malignant } & + & + & 0 \\ \text { malignant } & + & - & 0 \\ \text { malignant } & +++ & + & 3+ \\ \text { malignant } & +++ & + & 3+ \\ & & & \end{array}$

$\begin{array}{llll}+ & 77 & - & 3 \\ + & 79 & - & 3 \\ + & 68 & - & 3 \\ + & 74 & - & 3 \\ + & 36 & - & 2 \\ + & 43 & - & 3 \\ + & 39 & - & 2 \\ + & 32 & - & 2 \\ + & 56 & - & 3 \\ + & 62 & - & 3 \\ + & 55 & - & 3 \\ + & 62 & - & 3 \\ + & 65 & - & 3 \\ + & 38 & - & 2 \\ + & 45 & - & 3 \\ + & 52 & - & 3 \\ + & 62 & - & 3 \\ + & 54 & - & 3 \\ + & 49 & - & 3 \\ + & 36 & - & 2 \\ + & 41 & - & 3 \\ + & 53 & - & 3 \\ + & 60 & - & 3 \\ + & 56 & - & 3 \\ + & 47 & - & 3 \\ + & 49 & - & 3 \\ + & 37 & - & 2 \\ + & 42 & - & 3 \\ + & 44 & - & 3 \\ + & 39 & - & 3 \\ + & 34 & - & 2 \\ + & 53 & - & 3 \\ + & 53 & - & 3 \\ + & 4 & - & 2 \\ + & 33 \\ + & 25 & - & 2 \\ + & 53 & - & 3 \\ + & 42 & - & 3 \\ + & & & \end{array}$


Breast Breast Breast Breast Breast Breast Breast Breast Breast Breast Breast Breast Breast Breast Breast Breast Breast Breast Breast Breast Breast Breast Breast Breast Breast Breast Breast Breast Breast Breast

Breast Breast Breast Breast Breast Breast

Breast

Breast

Breast Breast
Invasive ductal carcinoma

Invasive ductal carcinoma

Invasive ductal carcinoma

Invasive ductal carcinoma

Invasive ductal carcinoma

Invasive ductal carcinoma

Invasive ductal carcinoma

Invasive ductal carcinoma

Invasive ductal carcinoma

Invasive ductal carcinoma

Invasive ductal carcinoma

Invasive ductal carcinoma

T1NOM0

T1NOMO

T1NOMO

T1NOMO

T1NOMO

T1NOMO

T1NOMO

T1NOMO

T1NOMO

T1NOMO

T1NOMO

T1NOMO

Invasive ductal carcinoma

T1NOMO

Invasive ductal carcinoma

T1NOMO

asive ductal carcinoma with necrc T1cN0M0

asive ductal carcinoma with necrc T1cNOM0

Invasive ductal carcinoma T1NOMO

asive ductal carcinoma with necrc T1N0M0

T1N0M0

Invasive ductal carcinoma T1NOMO

T1NOMO

Invasive ductal carcinoma

T1NOMO

T1NOMO

Invasive ductal carcinoma

T1NOMO

Invasive ductal carcinoma

T1NOMO

Invasive ductal carcinoma

T1NOMO

Invasive ductal carcinoma T1N0M0

ivasive ductal carcinoma (adenosi T1NOM0

vasive ductal carcinoma (adenosi T1N0M0

tal carcinoma (fibrous tissue and $k$ T1NOM0

tal carcinoma (fibrous tissue and $k$ T1NOMO

nvasive ductal carcinoma (sparse T1NOM0

Invasive lobular carcinoma T1N0M0

Invasive lobular carcinoma T1N0M0

nvasive ductal carcinoma (sparse T1N0M0

tal carcinoma (fibrous tissue and $k$ T1NOMO

tal carcinoma (fibrous tissue and $k$ T1NOMO

carcinoma (chronic inflammation ( T1NOMO

al carcinoma (fibrofatty tissue and T1NOMO

asive ductal carcinoma (breast tis؟ T1N0M0 malignant

malignant

malignant

malignant

malignant

malignant

malignant

malignant

malignant

malignant

malignant

malignant

malignant

malignant

malignant

malignant

malignant

malignant

malignant

malignant

malignant

malignant

malignant

malignant

malignant

malignant

malignant

malignant

malignant

malignant

malignant

malignant

malignant

malignant

malignant

malignant

malignant

malignant

malignant

malignant

$\begin{array}{ccc}- & - & 0 \\ - & - & 0 \\ - & - & 2+ \\ - & - & 2+ \\ ++ & - & 2+ \\ ++ & - & 2+ \\ + & + & 0 \\ + & + & 0 \\ +++ & - & 0 \\ +++ & - & 0 \\ ++ & + & 0 \\ ++ & + & 0 \\ - & - & 2+ \\ - & - & 2+ \\ - & - & 2+ \\ - & - & 2+ \\ - & - & 0 \\ - & - & 0 \\ +++ & +++ & 0 \\ ++ & ++ & 0 \\ ++ & ++ & 0 \\ - & - & 0 \\ - & - & 0 \\ - & * & 3+ \\ - & * & 3+ \\ - & - & 3+ \\ - & - & 3+ \\ * & * & - \\ * & * & - \\ * & * & 3+ \\ * & * & - \\ +++ & +++ & - \\ + & + & - \\ + & + & - \\ ++ & ++ & - \\ * & * & - \\ * & * & - \\ * & * & 0 \\ * & * & * \\ * & * & 0 \\ & & \end{array}$


156
Breast asive ductal carcinoma (breast tis؟ T1N0M0

Breast nvasive ductal carcinoma (sparse T1cNOMO

Breast nvasive ductal carcinoma (sparse T1cNOMO

Breast nvasive ductal carcinoma (sparse T1N0MO

Breast

Breast

Breast

Breast

Breast

Breast

Breast

Breast

Breast

$$
\begin{aligned}
& \text { Medullary carcinoma } \\
& \text { Medullary carcinoma }
\end{aligned}
$$

T1N0M0

T1NOM0

Papillary carcinoma

T1CNOM0

Papillary carcinoma

T1CNOM0

arcinoma (chronic inflammation of T1N0M0

arcinoma (chronic inflammation of T1NOMO

arcinoma (breast tis؟ T1NOMO

arcinoma (chronic inflammation of T1NOMO

Breast carcinoma (chronic inflammation ( T1NOMO

Breast carcinoma (chronic inflammation ( T1NOMO

Breast

Breast

Breast

Breast

Breast

Breast

Breast

Breast

Breast

Breast

Breast

Breast

Breast

Breast

Breast

Breast

Breast

Breast

Breast

Breast

Breast

Breast

Breast
Medullary carcinoma

T1NOMO

? ductal carcinoma (sparse) with r T1cN0M0

Medullary carcinoma (sparse) T1NOMO

Medullary carcinoma

Medullary carcinoma

T1NOM0

Medullary carcinoma

Adenosquamous carcinoma

T1CNOMO

T1CNOMO

T1NOM0

T1CNOMO Lipid-rich carcinoma

T1CNOMO

nvasive ductal carcinoma (sparse T1NOM0

tal carcinoma (fibrous tissue and $k$ T1NOMO

nvasive ductal carcinoma (sparse T1N0M0

sive ductal carcinoma (necrosis tis T1NOM0

Medullary carcinoma

Medullary carcinoma

T1NOMO

Medullary carcinoma

T1NOMO

Medullary carcinoma

T1NOMO

Invasive lobular carcinoma

T1NOMO

Invasive lobular carcinoma

T1NOMO

Invasive papillary carcinoma

T1NOMO

Invasive papillary carcinoma

$\begin{array}{cccc}\text { malignant } & * & * & * \\ \text { malignant } & - & - & 0 \\ \text { malignant } & - & - & 2+ \\ \text { malignant } & +++ & +++ & 0 \\ \text { malignant } & - & - & 0 \\ \text { malignant } & - & - & 0 \\ \text { malignant } & - & - & 0 \\ \text { malignant } & - & - & 0 \\ \text { malignant } & * & * & * \\ \text { malignant } & * & * & 0 \\ \text { malignant } & * & * & 0 \\ \text { malignant } & * & * & 0 \\ \text { malignant } & * & * & 0 \\ \text { malignant } & * & * & 0 \\ \text { malignant } & * & * & 0 \\ \text { malignant } & * & * & 0 \\ \text { malignant } & * & * & 0 \\ \text { malignant } & - & * & 0 \\ \text { malignant } & - & * & 0 \\ \text { malignant } & - & - & * \\ \text { malignant } & - & - & 0 \\ \text { malignant } & - & - & 0 \\ \text { malignant } & * & * & 0 \\ \text { malignant } & - & - & 0 \\ \text { malignant } & + & +++ & 0 \\ \text { malignant } & + & +++ & 0 \\ \text { malignant } & * & * & 0 \\ \text { malignant } & +++ & * & 0 \\ \text { malignant } & +++ & * & 0 \\ \text { malignant } & * & * & 0 \\ \text { malignant } & + & - & 3+ \\ \text { malignant } & * & * & * \\ \text { malignant } & - & - & 0 \\ \text { malignant } & - & - & 0 \\ \text { malignant } & - & - & 0 \\ \text { malignant } & - & - & 0 \\ \text { malignant } & + & - & 0 \\ \text { malignant } & + & - & 0 \\ \text { malignant } & +++ & - & 0 \\ \text { malignant } & +++ & - & 0\end{array}$

$\begin{array}{lll}38 & - & 2 \\ 31 & - & 2 \\ 55 & - & 3 \\ 58 & - & 3 \\ 43 & - & 3 \\ 65 & - & 3 \\ 34 & - & 2 \\ 37 & - & 2 \\ 42 & - & 3 \\ 51 & - & 3 \\ 28 & - & 2 \\ 26 & - & 2 \\ 21 & - & 2 \\ 33 & - & 2 \\ 43 & - & 3 \\ 51 & - & 3 \\ 34 & - & 2 \\ 41 & - & 3 \\ 46 & - & 3 \\ 53 & - & 3 \\ 58 & - & 3 \\ 49 & - & 3 \\ 36 & - & 2 \\ 44 & - & 3 \\ 51 & - & 3 \\ 66 & - & 3 \\ 62 & - & 3 \\ 67 & - & 3 \\ 58 & - & 3 \\ 33 & - & 2 \\ 31 & - & 2 \\ 28 & - & 2 \\ 49 & - & 3 \\ 50 & - & 3 \\ 28 & - & 2 \\ 30 & - & 2 \\ 47 & - & 3 \\ 38 & - & 2 \\ 44 & - & 3 \\ 52 & - & 3 \\ & & \end{array}$




$\begin{array}{llll}196 & 55 & \mathrm{~F} & \text { Breast } \\ 197 & 57 & \mathrm{~F} & \text { breast } \\ 198 & 65 & \mathrm{~F} & \text { breast } \\ 199 & 62 & \mathrm{~F} & \text { breast } \\ 200 & 58 & \mathrm{~F} & \text { breast } \\ 201 & 62 & \mathrm{~F} & \text { breast } \\ 202 & 48 & \mathrm{~F} & \text { breast } \\ 203 & 61 & \mathrm{~F} & \text { breast } \\ 204 & 59 & \mathrm{~F} & \text { breast } \\ 205 & 59 & \mathrm{~F} & \text { breast } \\ 206 & 57 & \mathrm{~F} & \text { breast } \\ 207 & 33 & \mathrm{~F} & \text { breast } \\ 208 & 48 & \mathrm{~F} & \text { breast } \\ 209 & 53 & \mathrm{~F} & \text { breast } \\ 210 & 47 & \mathrm{~F} & \text { breast } \\ 211 & 47 & \mathrm{~F} & \text { Breast } \\ 212 & 47 & \mathrm{~F} & \text { Breast } \\ 213 & 48 & \mathrm{~F} & \text { Breast } \\ 214 & 48 & \mathrm{~F} & \text { Breast } \\ 215 & 86 & \mathrm{~F} & \text { Breast } \\ 216 & 86 & \mathrm{~F} & \text { Breast } \\ 217 & 50 & \mathrm{~F} & \text { Breast } \\ 218 & 50 & \mathrm{~F} & \text { Breast } \\ 219 & 55 & \mathrm{~F} & \text { Breast } \\ 220 & 55 & \mathrm{~F} & \text { Breast } \\ 221 & 50 & \mathrm{~F} & \text { Breast } \\ 222 & 50 & \mathrm{~F} & \text { Breast } \\ 223 & 39 & \mathrm{~F} & \text { Breast } \\ 224 & 39 & \mathrm{~F} & \text { Breast } \\ 225 & 47 & \mathrm{~F} & \text { Breast } \\ 226 & 47 & \mathrm{~F} & \text { Breast } \\ 227 & 46 & \mathrm{~F} & \text { Breast } \\ 228 & 46 & \mathrm{~F} & \text { Breast } \\ 229 & 38 & \mathrm{~F} & \text { Breast } \\ 230 & 38 & \mathrm{~F} & \text { Breast } \\ 231 & 40 & \mathrm{~F} & \text { Breast } \\ & & & \end{array}$

Invasive ductal carcinoma infiltrative ductal carcinoma infiltrative ductal carcinoma infiltrative ductal carcinoma infiltrative ductal carcinoma infiltrative ductal carcinoma infiltrative ductal carcinoma infiltrative ductal carcinoma infiltrative ductal carcinoma infiltrative ductal carcinoma infiltrative ductal carcinoma infiltrative ductal carcinoma infiltrative ductal carcinoma infiltrative ductal carcinoma infiltrative ductal carcinoma Invasive ductal carcinoma Invasive ductal carcinoma Invasive ductal carcinoma Invasive ductal carcinoma Invasive ductal carcinoma Invasive ductal carcinoma Invasive ductal carcinoma Invasive ductal carcinoma Invasive ductal carcinoma Invasive ductal carcinoma

\section{Mucinous carcinoma}

Mucinous carcinoma

Invasive ductal carcinoma Invasive ductal carcinoma Invasive ductal carcinoma Invasive ductal carcinoma Invasive ductal carcinoma Invasive ductal carcinoma Invasive ductal carcinoma Invasive ductal carcinoma Invasive ductal carcinoma

$\begin{array}{lcccccc}\text { T1NOM0 } & 1--2 & \text { l } & \text { malignant } & +++ & * & 0 \\ \text { T1NOM0 } & 2 & \text { la } & \text { Malignant } & & & \\ \text { T1NOM0 } & 2 & \text { la } & \text { Malignant } & & & \\ \text { T1NOM0 } & 2 & \text { la } & \text { Malignant } & & & \\ \text { T1NOM0 } & 2 & \text { la } & \text { Malignant } & & & \\ \text { T1NOM0 } & 2 & \text { la } & \text { Malignant } & & & \\ \text { T1NOM0 } & 2 & \text { la } & \text { Malignant } & & & \\ \text { T1NOM0 } & 2 & \text { la } & \text { Malignant } & & & \\ \text { T1NOM0 } & 3 & \text { la } & \text { Malignant } & & & \\ \text { T1NOM0 } & 2--3 & \text { la } & \text { Malignant } & & & \\ \text { T1NOM0 } & 2--3 & \text { la } & \text { Malignant } & & & \\ \text { T1N1M0 } & 2 & \text { Ila } & \text { Malignant } & & & \\ \text { T2NOM0 } & 2 & \text { Ila } & \text { Malignant } & & & \\ \text { T2NOM0 } & 2--3 & \text { Ila } & \text { Malignant } & & & \\ \text { T2NOM0 } & 2--3 & \text { Ila } & \text { Malignant } & & & \\ \text { T2NOM0 } & 1 & \text { Ila } & \text { malignant } & - & - & 1+ \\ \text { T2NOM0 } & 1 & \text { Ila } & \text { malignant } & - & - & 1+ \\ \text { T2NOM0 } & 1 & \text { Ila } & \text { malignant } & ++ & ++ & - \\ \text { T2NOM0 } & 1 & \text { Ila } & \text { malignant } & ++ & ++ & - \\ \text { T2NOM0 } & 1 & \text { Ila } & \text { malignant } & +++ & - & 0 \\ \text { T2NOM0 } & 1 & \text { Ila } & \text { malignant } & +++ & - & 0 \\ \text { T2NOM0 } & 1 & \text { Ila } & \text { malignant } & +++ & +++ & 0 \\ \text { T2NOM0 } & 1 & \text { Ila } & \text { malignant } & +++ & +++ & 0 \\ \text { T2NOM0 } & 1 & \text { Ila } & \text { malignant } & +++ & - & 0 \\ \text { T2NOM0 } & 1 & \text { Ila } & \text { malignant } & +++ & - & 0 \\ \text { T2NOM0 } & 1 & \text { Ila } & \text { malignant } & ++ & ++ & 0 \\ \text { T2NOM0 } & 1 & \text { Ila } & \text { malignant } & ++ & ++ & 0 \\ \text { T2NOM0 } & 1 & \text { Ila } & \text { malignant } & - & * & 2+ \\ \text { T2NOM0 } & 1 & \text { Ila } & \text { malignant } & - & * & 2+ \\ \text { T2NOM0 } & 1 & \text { Ila } & \text { malignant } & ++ & * & 0 \\ \text { T2NOM0 } & 1 & \text { Ila } & \text { malignant } & ++ & * & 0 \\ \text { T2NOM0 } & 1 & \text { Ila } & \text { malignant } & ++ & ++ & 0 \\ \text { T2NOM0 } & 1 & \text { Ila } & \text { malignant } & ++ & ++ & 0 \\ \text { T2NOM0 } & 2 & \text { Ila } & \text { malignant } & - & - & - \\ \text { T2NOM0 } & 2 & \text { Ila } & \text { malignant } & - & - & 1+ \\ \text { T2NOM0 } & 2 & \text { Ila } & \text { malignant } & + & - & 2+ \\ & & & & & & \end{array}$

$\begin{array}{cccc}+ & 58 & - & 3 \\ + & 57 & - & 3 \\ + & 62 & - & 3 \\ + & 69 & - & 3 \\ + & 46 & - & 3 \\ + & 41 & - & 3 \\ + & 63 & - & 3 \\ + & 47 & - & 3 \\ + & 55 & - & 3 \\ + & 59 & - & 3 \\ + & 46 & - & 3 \\ + & 85 & + & 4 \\ + & 82 & + & 4 \\ + & 84 & + & 4 \\ + & 87 & + & 4 \\ + & 89 & + & 4 \\ + & 91 & + & 4 \\ + & 92 & + & 4 \\ + & 96 & + & 4 \\ + & 88 & + & 4 \\ + & 84 & + & 4 \\ + & 89 & + & 4 \\ + & 92 & + & 4 \\ + & 96 & + & 4 \\ + & 97 & + & 4 \\ + & 93 & + & 4 \\ + & 89 & + & 4 \\ + & 82 & + & 4 \\ + & 84 & + & 4 \\ + & 98 & + & 4 \\ + & 92 & + & 4 \\ + & 91 & + & 4 \\ + & 95 & + & 4 \\ + & 102 & + & 4 \\ + & 99 & + & 4 \\ + & 107 & + & 4\end{array}$




$\begin{array}{llll}232 & 40 & \mathrm{~F} & \text { Breast } \\ 233 & 49 & \mathrm{~F} & \text { Breast } \\ 234 & 49 & \mathrm{~F} & \text { Breast } \\ 235 & 45 & \mathrm{~F} & \text { Breast } \\ 236 & 45 & \mathrm{~F} & \text { Breast } \\ 237 & 45 & \mathrm{~F} & \text { Breast } \\ 238 & 45 & \mathrm{~F} & \text { Breast } \\ 239 & 55 & \mathrm{~F} & \text { Breast } \\ 240 & 55 & \mathrm{~F} & \text { Breast } \\ 241 & 50 & \mathrm{~F} & \text { Breast } \\ 242 & 50 & \mathrm{~F} & \text { Breast } \\ 243 & 45 & \mathrm{~F} & \text { Breast } \\ 244 & 45 & \mathrm{~F} & \text { Breast } \\ 245 & 61 & \mathrm{~F} & \text { Breast } \\ 246 & 33 & \mathrm{~F} & \text { Breast } \\ 247 & 52 & \mathrm{~F} & \text { Breast } \\ 248 & 52 & \mathrm{~F} & \text { Breast } \\ 249 & 51 & \mathrm{~F} & \text { Breast } \\ 250 & 51 & \mathrm{~F} & \text { Breast } \\ 251 & 52 & \mathrm{~F} & \text { Breast } \\ 252 & 54 & \mathrm{~F} & \text { Breast } \\ 253 & 54 & \mathrm{~F} & \text { Breast } \\ 254 & 32 & \mathrm{~F} & \text { Breast } \\ 255 & 32 & \mathrm{~F} & \text { Breast } \\ 256 & 31 & \mathrm{~F} & \text { Breast } \\ 257 & 31 & \mathrm{~F} & \text { Breast } \\ 258 & 58 & \mathrm{~F} & \text { Breast } \\ 259 & 58 & \mathrm{~F} & \text { Breast } \\ 260 & 45 & \mathrm{~F} & \text { Breast } \\ 261 & 45 & \mathrm{~F} & \text { Breast } \\ 262 & 51 & \mathrm{~F} & \text { Breast } \\ 263 & 51 & \mathrm{~F} & \text { Breast } \\ 264 & 64 & \mathrm{~F} & \text { Breast } \\ 265 & 39 & \mathrm{~F} & \text { Breast } \\ & & & \end{array}$

Invasive ductal carcinoma Invasive ductal carcinoma Invasive ductal carcinoma Invasive ductal carcinoma Invasive ductal carcinoma Invasive ductal carcinoma Invasive ductal carcinoma Invasive ductal carcinoma Invasive ductal carcinoma Invasive ductal carcinoma Invasive ductal carcinoma Invasive ductal carcinoma Invasive ductal carcinoma Invasive ductal carcinoma Invasive ductal carcinoma Invasive ductal carcinoma Invasive ductal carcinoma Invasive ductal carcinoma Invasive ductal carcinoma Invasive ductal carcinoma Invasive ductal carcinoma Invasive ductal carcinoma Invasive ductal carcinoma Invasive ductal carcinoma Invasive ductal carcinoma Invasive ductal carcinoma Invasive ductal carcinoma Invasive ductal carcinoma Invasive ductal carcinoma Invasive ductal carcinoma Invasive ductal carcinoma Invasive ductal carcinoma Invasive ductal carcinoma Invasive ductal carcinoma

\begin{tabular}{|c|c|c|c|c|c|c|}
\hline T2NOMO & 2 & Ila & malignant & + & - & - \\
\hline T2NOMO & 2 & Ila & malignant & ++ & ++ & - \\
\hline T2NOMO & 2 & Ila & malignant & ++ & ++ & - \\
\hline T2NOMO & 2 & Ila & malignant & + & ++ & - \\
\hline T2NOMO & 2 & Ila & malignant & + & ++ & - \\
\hline T2NOMO & 2 & Ila & malignant & +++ & - & - \\
\hline T2NOMO & 2 & Ila & malignant & +++ & - & - \\
\hline T2NOMO & 2 & Ila & malignant & +++ & - & - \\
\hline T2NOMO & 2 & Ila & malignant & +++ & - & - \\
\hline T2NOMO & 2 & Ila & malignant & + & - & - \\
\hline T2NOMO & 2 & Ila & malignant & + & - & - \\
\hline T2NOMO & 2 & Ila & malignant & ++ & ++ & - \\
\hline T2NOMO & 2 & Ila & malignant & ++ & ++ & - \\
\hline T2NOMO & 2 & Ila & malignant & ++ & + & - \\
\hline T2NOMO & 2 & Ila & malignant & ++ & ++ & - \\
\hline T2NOMO & 2 & Ila & malignant & +++ & - & - \\
\hline T2NOMO & 2 & Ila & malignant & +++ & - & - \\
\hline T2NOMO & 2 & Ila & malignant & +++ & + & - \\
\hline T2NOMO & 2 & Ila & malignant & +++ & + & - \\
\hline T2NOMO & 2 & Ila & malignant & + & - & $2+$ \\
\hline T2NOMO & 2 & Ila & malignant & ++ & + & - \\
\hline T2NOMO & 2 & Ila & malignant & ++ & + & - \\
\hline T2NOMO & 2 & Ila & malignant & ++ & ++ & - \\
\hline T2NOMO & 2 & Ila & malignant & ++ & ++ & - \\
\hline T2NOMO & 2 & Ila & malignant & - & - & - \\
\hline T2NOMO & 2 & Ila & malignant & - & - & - \\
\hline T2NOMO & 2 & Ila & malignant & ++ & ++ & - \\
\hline T2NOMO & 2 & Ila & malignant & ++ & ++ & - \\
\hline T2NOMO & 2 & Ila & malignant & ++ & +++ & $1+$ \\
\hline T2NOMO & 2 & Ila & malignant & ++ & +++ & - \\
\hline T2NOMO & 2 & Ila & malignant & - & - & $2+$ \\
\hline T2NOMO & 2 & Ila & malignant & - & - & $2+$ \\
\hline T2NOMO & 2 & Ila & malignant & - & - & - \\
\hline T2NOMO & 2 & Ila & malignant & - & - & $1+$ \\
\hline
\end{tabular}

$\begin{array}{cccc}+ & 103 & + & 4 \\ + & 112 & + & 4 \\ + & 109 & + & 4 \\ + & 97 & + & 4 \\ + & 124 & + & 4 \\ + & 116 & + & 4 \\ + & 125 & + & 4 \\ + & 109 & + & 4 \\ + & 117 & + & 4 \\ + & 108 & + & 4 \\ + & 104 & + & 4 \\ + & 115 & + & 4 \\ + & 108 & + & 4 \\ + & 103 & + & 4 \\ + & 101 & + & 4 \\ + & 106 & + & 4 \\ + & 121 & + & 4 \\ + & 113 & + & 4 \\ + & 104 & + & 4 \\ + & 107 & + & 4 \\ + & 113 & + & 4 \\ + & 115 & + & 4 \\ + & 98 & + & 4 \\ + & 99 & + & 4 \\ + & 95 & + & 4 \\ + & 107 & + & 4 \\ + & 114 & + & 4 \\ + & 109 & + & 4 \\ + & 124 & + & 4 \\ + & 107 & + & 4 \\ + & 116 & + & 4 \\ + & 109 & + & 4 \\ + & 96 & + & 4 \\ + & 99 & + & 4\end{array}$




$\begin{array}{llll}266 & 39 & \mathrm{~F} & \text { Breast } \\ 267 & 32 & \mathrm{~F} & \text { Breast } \\ 268 & 32 & \mathrm{~F} & \text { Breast } \\ 269 & 72 & \mathrm{~F} & \text { Breast } \\ 270 & 72 & \mathrm{~F} & \text { Breast } \\ 271 & 52 & \mathrm{~F} & \text { Breast } \\ 272 & 52 & \mathrm{~F} & \text { Breast } \\ 273 & 60 & \mathrm{~F} & \text { Breast } \\ 274 & 60 & \mathrm{~F} & \text { Breast } \\ 275 & 65 & \mathrm{~F} & \text { Breast } \\ 276 & 67 & \mathrm{~F} & \text { Breast } \\ 277 & 67 & \mathrm{~F} & \text { Breast } \\ 278 & 50 & \mathrm{~F} & \text { Breast } \\ 279 & 50 & \mathrm{~F} & \text { Breast } \\ 280 & 37 & \mathrm{~F} & \text { Breast } \\ 281 & 37 & \mathrm{~F} & \text { Breast } \\ 282 & 51 & \mathrm{~F} & \text { Breast } \\ 283 & 51 & \mathrm{~F} & \text { Breast } \\ 284 & 57 & \mathrm{~F} & \text { Breast } \\ 285 & 49 & \mathrm{~F} & \text { Breast } \\ 286 & 49 & \mathrm{~F} & \text { Breast } \\ 287 & 45 & \mathrm{~F} & \text { Breast } \\ 288 & 45 & \mathrm{~F} & \text { Breast } \\ 289 & 33 & \mathrm{~F} & \text { Breast } \\ 290 & 33 & \mathrm{~F} & \text { Breast } \\ 291 & 28 & \mathrm{~F} & \text { Breast } \\ 292 & 28 & \mathrm{~F} & \text { Breast } \\ 293 & 65 & \mathrm{~F} & \text { Breast } \\ 294 & 65 & \mathrm{~F} & \text { Breast } \\ 295 & 53 & \mathrm{~F} & \text { Breast } \\ 296 & 30 & \mathrm{~F} & \text { Breast } \\ 297 & 54 & \mathrm{~F} & \text { Breast } \\ 298 & 54 & \mathrm{~F} & \text { Breast } \\ 299 & 78 & \mathrm{~F} & \text { Breast } \\ & & & \end{array}$

Invasive ductal carcinoma Invasive ductal carcinoma Invasive ductal carcinoma Invasive ductal carcinoma Invasive ductal carcinoma Invasive ductal carcinoma Invasive ductal carcinoma Invasive ductal carcinoma Invasive ductal carcinoma Invasive ductal carcinoma Invasive ductal carcinoma Invasive ductal carcinoma Invasive ductal carcinoma Invasive ductal carcinoma Invasive ductal carcinoma Invasive ductal carcinoma Invasive ductal carcinoma Invasive ductal carcinoma Invasive ductal carcinoma Invasive ductal carcinoma Invasive ductal carcinoma Invasive ductal carcinoma Invasive ductal carcinoma Invasive ductal carcinoma Invasive ductal carcinoma Invasive ductal carcinoma Invasive ductal carcinoma Invasive ductal carcinoma Invasive ductal carcinoma Invasive ductal carcinoma Invasive ductal carcinoma Invasive ductal carcinoma Invasive ductal carcinoma Invasive ductal carcinoma

$\begin{array}{llllccc}\text { T2NOM0 } & 2 & \text { Ila } & \text { malignant } & - & - & 1+ \\ \text { T2NOM0 } & 2 & \text { Ila } & \text { malignant } & - & - & - \\ \text { T2NOM0 } & 2 & \text { Ila } & \text { malignant } & - & - & - \\ \text { T2NOM0 } & 2 & \text { Ila } & \text { malignant } & +++ & ++ & - \\ \text { T2NOM0 } & 2 & \text { Ila } & \text { malignant } & +++ & ++ & - \\ \text { T2NOM0 } & 2 & \text { Ila } & \text { malignant } & ++ & ++ & - \\ \text { T2NOM0 } & 2 & \text { Ila } & \text { malignant } & ++ & ++ & - \\ \text { T2NOM0 } & 2 & \text { Ila } & \text { malignant } & +++ & ++ & - \\ \text { T2NOM0 } & 2 & \text { Ila } & \text { malignant } & +++ & ++ & - \\ \text { T2NOM0 } & 2 & \text { Ila } & \text { malignant } & - & - & 1+ \\ \text { T2NOM0 } & 2 & \text { Ila } & \text { malignant } & + & - & 2+ \\ \text { T2NOM0 } & 2 & \text { Ila } & \text { malignant } & + & - & 3+ \\ \text { T2NOM0 } & 2 & \text { Ila } & \text { malignant } & + & - & - \\ \text { T2NOM0 } & 2 & \text { Ila } & \text { malignant } & + & - & - \\ \text { T2NOM0 } & 2 & \text { Ila } & \text { malignant } & +++ & ++ & - \\ \text { T2NOM0 } & 2 & \text { Ila } & \text { malignant } & +++ & ++ & - \\ \text { T2NOM0 } & 2 & \text { Ila } & \text { malignant } & ++ & ++ & - \\ \text { T2NOM0 } & 2 & \text { Ila } & \text { malignant } & ++ & ++ & - \\ \text { T2NOM0 } & 2 & \text { Ila } & \text { malignant } & ++ & + & - \\ \text { T2NOM0 } & 2 & \text { Ila } & \text { malignant } & - & - & 3+ \\ \text { T2NOM0 } & 2 & \text { Ila } & \text { malignant } & - & - & 3+ \\ \text { T2NOM0 } & 2 & \text { Ila } & \text { malignant } & ++ & - & - \\ \text { T2NOM0 } & 2 & \text { Ila } & \text { malignant } & ++ & - & - \\ \text { T2NOM0 } & 2 & \text { Ila } & \text { malignant } & ++ & + & - \\ \text { T2NOM0 } & 2 & \text { Ila } & \text { malignant } & ++ & + & - \\ \text { T2NOM0 } & 2 & \text { Ila } & \text { malignant } & +++ & + & - \\ \text { T2NOM0 } & 2 & \text { Ila } & \text { malignant } & +++ & + & - \\ \text { T2NOM0 } & 2 & \text { Ila } & \text { malignant } & +++ & - & - \\ \text { T2NOM0 } & 2 & \text { Ila } & \text { malignant } & +++ & - & - \\ \text { T2NOM0 } & 2 & \text { Ila } & \text { malignant } & - & - & - \\ \text { T2NOM0 } & 2 & \text { Ila } & \text { malignant } & + & + & - \\ \text { T2NOM0 } & 2 & \text { Ila } & \text { malignant } & ++ & ++ & - \\ \text { T2NOM0 } & 2 & \text { Ila } & \text { malignant } & ++ & ++ & - \\ \text { T2NOM0 } & 2 & \text { Ila } & \text { malignant } & ++ & - & -\end{array}$

$\begin{array}{cccc}+ & 103 & + & 4 \\ + & 112 & + & 4 \\ + & 108 & + & 4 \\ + & 102 & + & 4 \\ + & 117 & + & 4 \\ + & 113 & + & 4 \\ + & 125 & + & 4 \\ + & 104 & + & 4 \\ + & 98 & + & 4 \\ + & 95 & + & 4 \\ + & 112 & + & 4 \\ + & 124 & + & 4 \\ + & 97 & + & 4 \\ + & 99 & + & 4 \\ + & 91 & + & 4 \\ + & 121 & + & 4 \\ + & 126 & + & 4 \\ + & 113 & + & 4 \\ + & 118 & + & 4 \\ + & 123 & + & 4 \\ + & 103 & + & 4 \\ + & 109 & + & 4 \\ + & 98 & + & 4 \\ + & 96 & + & 4 \\ + & 156 & + & 4 \\ + & 164 & + & 4 \\ + & 171 & + & 4 \\ + & 154 & + & 4 \\ + & 127 & + & 4 \\ + & 98 & + & 4 \\ + & 128 & + & 4 \\ + & 134 & + & 4 \\ + & 128 & + & 4 \\ + & 119 & + & 4\end{array}$




$\begin{array}{llll}300 & 78 & \mathrm{~F} & \text { Breast } \\ 301 & 58 & \mathrm{~F} & \text { Breast } \\ 302 & 58 & \mathrm{~F} & \text { Breast } \\ 303 & 52 & \mathrm{~F} & \text { Breast } \\ 304 & 52 & \mathrm{~F} & \text { Breast } \\ 305 & 44 & \mathrm{~F} & \text { Breast } \\ 306 & 47 & \mathrm{~F} & \text { Breast } \\ 307 & 47 & \mathrm{~F} & \text { Breast } \\ 308 & 30 & \mathrm{~F} & \text { Breast } \\ 309 & 30 & \mathrm{~F} & \text { Breast } \\ 310 & 45 & \mathrm{~F} & \text { Breast } \\ 311 & 45 & \mathrm{~F} & \text { Breast } \\ 312 & 48 & \mathrm{~F} & \text { Breast } \\ 313 & 48 & \mathrm{~F} & \text { Breast } \\ 314 & 49 & \mathrm{~F} & \text { Breast } \\ 315 & 49 & \mathrm{~F} & \text { Breast } \\ 316 & 65 & \mathrm{~F} & \text { Breast } \\ 317 & 65 & \mathrm{~F} & \text { Breast } \\ 318 & 43 & \mathrm{~F} & \text { Breast } \\ 319 & 43 & \mathrm{~F} & \text { Breast } \\ 320 & 46 & \mathrm{~F} & \text { Breast } \\ 321 & 46 & \mathrm{~F} & \text { Breast } \\ 322 & 43 & \mathrm{~F} & \text { Breast } \\ 323 & 43 & \mathrm{~F} & \text { Breast } \\ 324 & 56 & \mathrm{~F} & \text { Breast } \\ 325 & 56 & \mathrm{~F} & \text { Breast } \\ 326 & 47 & \mathrm{~F} & \text { Breast } \\ 327 & 47 & \mathrm{~F} & \text { Breast } \\ 328 & 40 & \mathrm{~F} & \text { Breast } \\ 329 & 40 & \mathrm{~F} & \text { Breast } \\ 330 & 46 & \mathrm{~F} & \text { Breast } \\ 331 & 46 & \mathrm{~F} & \text { Breast } \\ 332 & 47 & \mathrm{~F} & \text { Breast } \\ 333 & 47 & \mathrm{~F} & \text { Breast } \\ & & & \end{array}$

\begin{tabular}{|c|c|c|c|c|c|c|}
\hline Invasive ductal carcinoma & T2NOMO & 2 & Ila & malignant & ++ & - \\
\hline Invasive ductal carcinoma & T2NOMO & 2 & Ila & malignant & - & - \\
\hline Invasive ductal carcinoma & T2NOMO & 2 & Ila & malignant & - & - \\
\hline Invasive ductal carcinoma & T2NOMO & 2 & Ila & malignant & ++ & ++ \\
\hline Invasive ductal carcinoma & T2NOMO & 2 & Ila & malignant & ++ & ++ \\
\hline Invasive ductal carcinoma & T2NOMO & 2 & Ila & malignant & - & - \\
\hline Invasive ductal carcinoma & T2NOMO & 2 & Ila & malignant & - & - \\
\hline Invasive ductal carcinoma & T2NOMO & 2 & Ila & malignant & - & - \\
\hline Invasive ductal carcinoma & T2NOMO & 2 & Ila & malignant & + & + \\
\hline Invasive ductal carcinoma & T2NOMO & 2 & Ila & malignant & + & + \\
\hline asive ductal carcinoma (sparse & T2NOMO & 2 & Ila & malignant & + & + \\
\hline asive ductal carcinoma (sparse & T2NOMO & 2 & Ila & malignant & + & + \\
\hline Invasive ductal carcinoma & T2NOMO & 2 & Ila & malignant & + & - \\
\hline Invasive ductal carcinoma & T2NOMO & 2 & Ila & malignant & + & - \\
\hline Invasive ductal carcinoma & T2NOMO & 2 & Ila & malignant & - & - \\
\hline Invasive ductal carcinoma & T2NOMO & 2 & Ila & malignant & - & - \\
\hline Mucinous carcinoma & T2NOMO & 2 & $\mathrm{Ila}$ & malignant & ++ & ++ \\
\hline Mucinous carcinoma & T2NOMO & 2 & $\mathrm{Ila}$ & malignant & ++ & ++ \\
\hline Invasive ductal carcinoma & T2NOMO & 2 & $\mathrm{Ila}$ & malignant & ++ & ++ \\
\hline Invasive ductal carcinoma & T2NOMO & 2 & Ila & lignant & ++ & ++ \\
\hline Invasive ductal carcinoma & T2NOMO & 2 & Ila & malignant & - & +++ \\
\hline Invasive ductal carcinoma & T2NOMO & 2 & Ila & malignant & - & +++ \\
\hline Invasive ductal carcinoma & T2NOMO & 2 & Ila & malignant & * & +++ \\
\hline Invasive ductal carcinoma & T2NOMO & 2 & Ila & malignant & * & +++ \\
\hline Invasive ductal carcinoma & T2NOMO & 2 & Ila & malignant & * & + \\
\hline Invasive ductal carcinoma & T2NOMO & 2 & Ila & malignant & * & + \\
\hline Invasive ductal carcinoma & T2NOMO & 2 & Ila & malignant & * & +++ \\
\hline Invasive ductal carcinoma & T2NOMO & 2 & Ila & malignant & * & +++ \\
\hline Invasive ductal carcinoma & T2NOMO & 2 & Ila & malignant & + & + \\
\hline Invasive ductal carcinoma & T2NOMO & 2 & Ila & malignant & + & + \\
\hline tal carcinoma with intraductal c & T2NOMO & 2 & Ila & malignant & - & - \\
\hline tal carcinoma with intraductal c & T2NOMO & 2 & Ila & malignant & - & - \\
\hline Invasive ductal carcinoma & T2NOMO & 2 & Ila & malignant & - & +++ \\
\hline Invasive ductal carcinoma & T2NOMO & 2 & Ila & malignant & - & +++ \\
\hline
\end{tabular}

$\begin{array}{cccc}+ & 126 & + & 4 \\ + & 131 & + & 4 \\ + & 125 & + & 4 \\ + & 146 & + & 4 \\ + & 138 & + & 4 \\ + & 128 & + & 4 \\ + & 121 & + & 4 \\ + & 129 & + & 4 \\ + & 109 & + & 4 \\ + & 103 & + & 4 \\ + & 115 & + & 4 \\ + & 126 & + & 4 \\ + & 98 & + & 4 \\ + & 97 & + & 4 \\ + & 120 & + & 4 \\ + & 132 & + & 4 \\ + & 127 & + & 4 \\ + & 119 & + & 4 \\ + & 116 & + & 4 \\ + & 137 & + & 4 \\ + & 132 & + & 4 \\ + & 126 & + & 4 \\ + & 108 & + & 4 \\ + & 105 & + & 4 \\ + & 98 & + & 4 \\ + & 96 & + & 4 \\ + & 112 & + & 4 \\ + & 118 & + & 4 \\ + & 109 & + & 4 \\ + & 102 & + & 4 \\ + & 105 & + & 4 \\ + & 114 & + & 4 \\ + & 116 & + & 4 \\ + & 122 & + & 4\end{array}$




$\begin{array}{llll}334 & 66 & \mathrm{~F} & \text { Breast } \\ 335 & 66 & \mathrm{~F} & \text { Breast } \\ 336 & 47 & \mathrm{~F} & \text { Breast } \\ 337 & 47 & \mathrm{~F} & \text { Breast } \\ 338 & 47 & \mathrm{~F} & \text { Breast } \\ 339 & 47 & \mathrm{~F} & \text { Breast } \\ 340 & 49 & \mathrm{~F} & \text { Breast } \\ 341 & 49 & \mathrm{~F} & \text { Breast } \\ 342 & 50 & \mathrm{~F} & \text { Breast } \\ 343 & 50 & \mathrm{~F} & \text { Breast } \\ 344 & 50 & \mathrm{~F} & \text { Breast } \\ 345 & 50 & \mathrm{~F} & \text { Breast } \\ 346 & 42 & \mathrm{~F} & \text { Breast } \\ 347 & 42 & \mathrm{~F} & \text { Breast } \\ 348 & 44 & \mathrm{~F} & \text { Breast } \\ 349 & 44 & \mathrm{~F} & \text { Breast } \\ 350 & 60 & \mathrm{~F} & \text { Breast } \\ 351 & 60 & \mathrm{~F} & \text { Breast } \\ 352 & 48 & \mathrm{~F} & \text { Breast } \\ 353 & 48 & \mathrm{~F} & \text { Breast } \\ 354 & 69 & \mathrm{~F} & \text { Breast } \\ 355 & 69 & \mathrm{~F} & \text { Breast } \\ 356 & 65 & \mathrm{~F} & \text { Breast } \\ 357 & 65 & \mathrm{~F} & \text { Breast } \\ 358 & 43 & \mathrm{~F} & \text { Breast } \\ 359 & 43 & \mathrm{~F} & \text { Breast } \\ 360 & 43 & \mathrm{~F} & \text { Breast } \\ 361 & 43 & \mathrm{~F} & \text { Breast } \\ 362 & 57 & \mathrm{~F} & \text { Breast } \\ 363 & 57 & \mathrm{~F} & \text { Breast } \\ 364 & 45 & \mathrm{~F} & \text { Breast } \\ 365 & 47 & \mathrm{~F} & \text { Breast } \\ 366 & 47 & \mathrm{~F} & \text { Breast } \\ 367 & 47 & \mathrm{~F} & \text { Breast } \\ & & & \end{array}$

\begin{tabular}{|c|c|c|c|c|c|c|}
\hline Invasive ductal carcinoma & T2NOMO & 2 & Ila & malignant & - & ++ \\
\hline Invasive ductal carcinoma & T2NOMO & 2 & Ila & malignant & - & ++ \\
\hline Invasive ductal carcinoma & T2NOMO & 2 & Ila & malignant & - & ++ \\
\hline Invasive ductal carcinoma & T2NOMO & 2 & Ila & malignant & * & ++ \\
\hline Invasive ductal carcinoma & T2NOMO & 2 & Ila & malignant & * & + \\
\hline Invasive ductal carcinoma & T2NOMO & 2 & Ila & malignant & * & + \\
\hline Invasive ductal carcinoma & T2NOMO & 2 & Ila & malignant & * & + \\
\hline Invasive ductal carcinoma & T2NOMO & 2 & Ila & malignant & * & - \\
\hline Invasive ductal carcinoma & T2NOMO & 2 & Ila & malignant & - & - \\
\hline Invasive ductal carcinoma & T2NOMO & 2 & Ila & malignant & * & - \\
\hline Invasive ductal carcinoma & T2NOMO & 2 & Ila & malignant & ++ & - \\
\hline Invasive ductal carcinoma & T2NOMO & 2 & Ila & malignant & ++ & - \\
\hline Invasive ductal carcinoma & T2NOMO & 2 & Ila & malignant & + & - \\
\hline Invasive ductal carcinoma & T2NOMO & 2 & Ila & malignant & + & - \\
\hline Invasive ductal carcinoma & T2NOMO & 2 & Ila & malignant & ++ & + \\
\hline Invasive ductal carcinoma & T2NOMO & 2 & Ila & malignant & ++ & + \\
\hline Invasive ductal carcinoma & T2NOMO & 2 & Ila & malignant & - & - \\
\hline Invasive ductal carcinoma & T2NOMO & 2 & Ila & malignant & - & - \\
\hline Invasive ductal carcinoma & T2NOMO & 2 & Ila & malignant & +++ & + \\
\hline Invasive ductal carcinoma & T2NOMO & 2 & Ila & malignant & +++ & + \\
\hline Invasive ductal carcinoma & T2NOMO & 2 & Ila & malignant & +++ & +++ \\
\hline Invasive ductal carcinoma & T2NOMO & 2 & Ila & malignant & +++ & +++ \\
\hline Invasive ductal carcinoma & T2NOMO & 2 & Ila & malignant & - & - \\
\hline Invasive ductal carcinoma & T2NOMO & 2 & Ila & malignant & - & - \\
\hline Invasive ductal carcinoma & T2NOMO & 2 & Ila & malignant & ++ & + \\
\hline Invasive ductal carcinoma & T2NOMO & 2 & Ila & malignant & ++ & + \\
\hline Invasive ductal carcinoma & T2NOMO & 2 & Ila & malignant & - & - \\
\hline Invasive ductal carcinoma & T2NOMO & 2 & Ila & malignant & - & - \\
\hline Invasive ductal carcinoma & T2NOMO & 2 & Ila & malignant & ++ & ++ \\
\hline Invasive ductal carcinoma & T2NOMO & 2 & Ila & malignant & ++ & ++ \\
\hline Invasive ductal carcinoma & T2NOMO & 2 & Ila & malignant & + & + \\
\hline Invasive ductal carcinoma & T2NOMO & 2 & Ila & malignant & ++ & ++ \\
\hline Invasive ductal carcinoma & T2NOMO & 2 & Ila & malignant & ++ & ++ \\
\hline Invasive ductal carcinoma & T2NOMO & 2 & Ila & malignant & - & - \\
\hline
\end{tabular}

$\begin{array}{cccc}+ & 110 & + & 4 \\ + & 127 & + & 4 \\ + & 96 & + & 4 \\ + & 109 & + & 4 \\ + & 105 & + & 4 \\ + & 92 & + & 4 \\ + & 156 & + & 4 \\ + & 126 & + & 4 \\ + & 163 & + & 4 \\ + & 102 & + & 4 \\ + & 134 & + & 4 \\ + & 137 & + & 4 \\ + & 119 & + & 4 \\ + & 97 & + & 4 \\ + & 94 & + & 4 \\ + & 132 & + & 4 \\ + & 141 & + & 4 \\ + & 135 & + & 4 \\ + & 146 & + & 4 \\ + & 139 & + & 4 \\ + & 167 & + & 4 \\ + & 182 & + & 4 \\ + & 175 & + & 4 \\ + & 79 & + & 4 \\ + & 67 & + & 4 \\ + & 174 & + & 4 \\ + & 86 & + & 4 \\ + & 72 & + & 4 \\ + & 68 & + & 4 \\ + & 152 & + & 4 \\ + & 127 & + & 4 \\ + & 138 & + & 4 \\ + & 153 & + & 4 \\ + & & & 4\end{array}$




$\begin{array}{llll}368 & 47 & \mathrm{~F} & \text { Breast } \\ 369 & 39 & \mathrm{~F} & \text { Breast } \\ 370 & 39 & \mathrm{~F} & \text { Breast } \\ 371 & 45 & \mathrm{~F} & \text { Breast } \\ 372 & 45 & \mathrm{~F} & \text { Breast } \\ 373 & 46 & \mathrm{~F} & \text { Breast } \\ 374 & 46 & \mathrm{~F} & \text { Breast } \\ 375 & 47 & \mathrm{~F} & \text { Breast } \\ 376 & 47 & \mathrm{~F} & \text { Breast } \\ 377 & 46 & \mathrm{~F} & \text { Breast } \\ 378 & 46 & \mathrm{~F} & \text { Breast } \\ 379 & 48 & \mathrm{~F} & \text { Breast } \\ 380 & 48 & \mathrm{~F} & \text { Breast } \\ 381 & 47 & \mathrm{~F} & \text { Breast } \\ 382 & 47 & \mathrm{~F} & \text { Breast } \\ 383 & 44 & \mathrm{~F} & \text { Breast } \\ 384 & 44 & \mathrm{~F} & \text { Breast } \\ 385 & 52 & \mathrm{~F} & \text { Breast } \\ 386 & 52 & \mathrm{~F} & \text { Breast } \\ 387 & 59 & \mathrm{~F} & \text { Breast } \\ 388 & 59 & \mathrm{~F} & \text { Breast } \\ 389 & 40 & \mathrm{~F} & \text { Breast } \\ 390 & 40 & \mathrm{~F} & \text { Breast } \\ 391 & 45 & \mathrm{~F} & \text { Breast } \\ 392 & 45 & \mathrm{~F} & \text { Breast } \\ 393 & 43 & \mathrm{~F} & \text { Breast } \\ 394 & 43 & \mathrm{~F} & \text { Breast } \\ 395 & 34 & \mathrm{~F} & \text { Breast } \\ 396 & 34 & \mathrm{~F} & \text { Breast } \\ 397 & 49 & \mathrm{~F} & \text { Breast } \\ 398 & 49 & \mathrm{~F} & \text { Breast } \\ 399 & 51 & \mathrm{~F} & \text { Breast } \\ 400 & 51 & \mathrm{~F} & \text { Breast } \\ 401 & 49 & \mathrm{~F} & \text { Breast } \\ & & & \end{array}$

\begin{tabular}{|c|c|c|c|c|c|c|}
\hline Invasive ductal carcinoma & T2NOMO & 2 & Ila & malignant & - & - \\
\hline Invasive ductal carcinoma & T2NOMO & 2 & Ila & malignant & ++ & + \\
\hline Invasive ductal carcinoma & T2NOMO & 2 & Ila & malignant & ++ & + \\
\hline Invasive ductal carcinoma & T2NOMO & 2 & Ila & malignant & - & - \\
\hline Invasive ductal carcinoma & T2NOMO & 2 & Ila & malignant & - & - \\
\hline Invasive ductal carcinoma & T2NOMO & 2 & Ila & malignant & - & - \\
\hline Invasive ductal carcinoma & T2NOMO & 2 & Ila & malignant & - & - \\
\hline Invasive ductal carcinoma & T2NOMO & 2 & Ila & malignant & +++ & +++ \\
\hline Invasive ductal carcinoma & T2NOMO & 2 & Ila & malignant & +++ & +++ \\
\hline Invasive ductal carcinoma & T2NOMO & 2 & Ila & malignant & - & - \\
\hline Invasive ductal carcinoma & T2NOMO & 2 & Ila & malignant & - & - \\
\hline Invasive ductal carcinoma & T2NOMO & 2 & Ila & malignant & - & - \\
\hline Invasive ductal carcinoma & T2NOMO & 2 & Ila & malignant & - & - \\
\hline Invasive ductal carcinoma & T2NOMO & 2 & Ila & malignant & - & - \\
\hline Invasive ductal carcinoma & T2NOMO & 2 & Ila & malignant & - & - \\
\hline Invasive ductal carcinoma & T2NOMO & 2 & Ila & malignant & ++ & ++ \\
\hline Invasive ductal carcinoma & T2NOMO & 2 & Ila & malignant & ++ & ++ \\
\hline Invasive ductal carcinoma & T2NOMO & 2 & Ila & malignant & + & - \\
\hline Invasive ductal carcinoma & T2NOMO & 2 & Ila & malignant & + & - \\
\hline Invasive ductal carcinoma & T2NOMO & 2 & Ila & malignant & ++ & ++ \\
\hline Invasive ductal carcinoma & T2NOMO & 2 & Ila & malignant & ++ & ++ \\
\hline Invasive ductal carcinoma & T2NOMO & 2 & Ila & malignant & +++ & +++ \\
\hline Invasive ductal carcinoma & T2NOMO & 2 & Ila & malignant & +++ & +++ \\
\hline Invasive ductal carcinoma & T2NOMO & 2 & Ila & malignant & - & - \\
\hline Invasive ductal carcinoma & T2NOMO & 2 & Ila & malignant & - & - \\
\hline Invasive ductal carcinoma & T2NOMO & 2 & Ila & malignant & +++ & +++ \\
\hline Invasive ductal carcinoma & T2NOMO & 2 & Ila & malignant & +++ & +++ \\
\hline Invasive ductal carcinoma & T2NOMO & 2 & Ila & malignant & ++ & + \\
\hline Invasive ductal carcinoma & T2NOMO & 2 & Ila & malignant & ++ & + \\
\hline Invasive ductal carcinoma & T2NOMO & 2 & Ila & malignant & + & + \\
\hline Invasive ductal carcinoma & T2NOMO & 2 & Ila & malignant & + & + \\
\hline Invasive ductal carcinoma & T2NOMO & 2 & Ila & malignant & + & - \\
\hline Invasive ductal carcinoma & T2NOMO & 2 & Ila & malignant & + & + \\
\hline Invasive ductal carcinoma & T2NOMO & 2 & Ila & malignant & + & ++ \\
\hline
\end{tabular}

$\begin{array}{cccc}+ & 68 & + & 4 \\ + & 89 & + & 4 \\ + & 99 & + & 4 \\ + & 103 & + & 4 \\ + & 107 & + & 4 \\ + & 89 & + & 4 \\ + & 86 & + & 4 \\ + & 187 & + & 4 \\ + & 176 & + & 4 \\ + & 117 & + & 4 \\ + & 118 & + & 4 \\ + & 87 & + & 4 \\ + & 65 & + & 4 \\ + & 173 & + & 4 \\ + & 182 & + & 4 \\ + & 97 & + & 4 \\ + & 96 & + & 4 \\ + & 87 & + & 4 \\ + & 92 & + & 4 \\ + & 91 & + & 4 \\ + & 103 & + & 4 \\ + & 104 & + & 4 \\ + & 117 & + & 4 \\ + & 96 & + & 4 \\ + & 87 & + & 4 \\ + & 157 & + & 4 \\ + & 135 & + & 4 \\ + & 99 & + & 4 \\ + & 101 & + & 4 \\ + & 89 & + & 4 \\ + & 81 & ++ & 4 \\ + & 103 & + & 4 \\ + & 108 & + & 4 \\ + & 125 & + & 4 \\ + & & & \end{array}$




$\begin{array}{llll}402 & 49 & \mathrm{~F} & \text { Breast } \\ 403 & 61 & \mathrm{~F} & \text { Breast } \\ 404 & 61 & \mathrm{~F} & \text { Breast } \\ 405 & 35 & \mathrm{~F} & \text { Breast } \\ 406 & 42 & \mathrm{~F} & \text { Breast } \\ 407 & 69 & \mathrm{~F} & \text { Breast } \\ 408 & 69 & \mathrm{~F} & \text { Breast } \\ 409 & 51 & \mathrm{~F} & \text { Breast } \\ 410 & 51 & \mathrm{~F} & \text { Breast } \\ 411 & 46 & \mathrm{~F} & \text { Breast } \\ 412 & 46 & \mathrm{~F} & \text { Breast } \\ 413 & 46 & \mathrm{~F} & \text { Breast } \\ 414 & 46 & \mathrm{~F} & \text { Breast } \\ 415 & 50 & \mathrm{~F} & \text { Breast } \\ 416 & 50 & \mathrm{~F} & \text { Breast } \\ 417 & 47 & \mathrm{~F} & \text { Breast } \\ 418 & 47 & \mathrm{~F} & \text { Breast } \\ 419 & 48 & \mathrm{~F} & \text { Breast } \\ 420 & 33 & \mathrm{~F} & \text { Breast } \\ 421 & 33 & \mathrm{~F} & \text { Breast } \\ 422 & 55 & \mathrm{~F} & \text { Breast } \\ 423 & 55 & \mathrm{~F} & \text { Breast } \\ 424 & 48 & \mathrm{~F} & \text { Breast } \\ 425 & 48 & \mathrm{~F} & \text { Breast } \\ 426 & 49 & \mathrm{~F} & \text { Breast } \\ 427 & 49 & \mathrm{~F} & \text { Breast } \\ 428 & 35 & \mathrm{~F} & \text { Breast } \\ 429 & 35 & \mathrm{~F} & \text { Breast } \\ 430 & 60 & \mathrm{~F} & \text { Breast } \\ 431 & 60 & \mathrm{~F} & \text { Breast } \\ 432 & 45 & \mathrm{~F} & \text { Breast } \\ 433 & 45 & \mathrm{~F} & \text { Breast } \\ 434 & 60 & \mathrm{~F} & \text { Breast } \\ 435 & 60 & \mathrm{~F} & \text { Breast } \\ & & & \end{array}$

\begin{tabular}{|c|c|c|c|c|c|c|}
\hline Invasive ductal carcinoma & T2N0MO & 2 & Ila & malignant & + & ++ \\
\hline Invasive ductal carcinoma & T2NOMO & 2 & Ila & malignant & ++ & + \\
\hline Invasive ductal carcinoma & T2NOMO & 2 & Ila & malignant & ++ & + \\
\hline Invasive ductal carcinoma & T2NOMO & 2 & Ila & malignant & +++ & +++ \\
\hline Invasive ductal carcinoma & T2NOMO & 2 & Ila & malignant & - & - \\
\hline Invasive ductal carcinoma & T2NOMO & 2 & Ila & malignant & - & - \\
\hline Invasive ductal carcinoma & T2NOMO & 2 & Ila & malignant & - & - \\
\hline Invasive ductal carcinoma & T2NOMO & 2 & Ila & malignant & - & - \\
\hline Invasive ductal carcinoma & T2NOMO & 2 & Ila & malignant & - & - \\
\hline Invasive ductal carcinoma & T2NOMO & 2 & Ila & malignant & - & - \\
\hline Invasive ductal carcinoma & T2NOMO & 2 & Ila & malignant & - & - \\
\hline Invasive ductal carcinoma & T2NOMO & 2 & Ila & malignant & - & - \\
\hline Invasive ductal carcinoma & T2NOMO & 2 & Ila & malignant & - & - \\
\hline Invasive ductal carcinoma & T2NOMO & 2 & Ila & malignant & + & - \\
\hline Invasive ductal carcinoma & T2NOMO & 2 & Ila & malignant & + & - \\
\hline Invasive ductal carcinoma & T2NOMO & 2 & Ila & malignant & +++ & +++ \\
\hline Invasive ductal carcinoma & T2NOMO & 2 & Ila & malignant & +++ & +++ \\
\hline Invasive ductal carcinoma & T2NOMO & 2 & Ila & malignant & ++ & ++ \\
\hline Invasive ductal carcinoma & T2NOMO & 2 & Ila & malignant & ++ & - \\
\hline Invasive ductal carcinoma & T2NOMO & 2 & Ila & malignant & ++ & - \\
\hline Invasive ductal carcinoma & T2NOMO & 2 & Ila & & - & - \\
\hline Invasive ductal carcinoma & T2NOMO & 2 & Ila & malignant & - & - \\
\hline Invasive ductal carcinoma & T2NOMO & 2 & Ila & malignant & + & ++ \\
\hline Invasive ductal carcinoma & T2NOMO & 2 & Ila & malignant & + & ++ \\
\hline Invasive ductal carcinoma & T2NOMO & 2 & Ila & malignant & * & * \\
\hline Invasive ductal carcinoma & T2NOMO & 2 & Ila & malignant & +++ & +++ \\
\hline Invasive ductal carcinoma & T2NOMO & 2 & Ila & malignant & +++ & ++ \\
\hline Invasive ductal carcinoma & T2NOMO & 2 & Ila & malignant & +++ & ++ \\
\hline Invasive ductal carcinoma & T2NOMO & 2 & Ila & malignant & +++ & - \\
\hline Invasive ductal carcinoma & T2NOMO & 2 & Ila & malignant & +++ & - \\
\hline Invasive ductal carcinoma & T2NOMO & 2 & Ila & malignant & ++ & +++ \\
\hline Invasive ductal carcinoma & T2NOMO & 2 & Ila & malignant & ++ & +++ \\
\hline Invasive ductal carcinoma & T2NOMO & 2 & तार & malignant & +++ & ++ \\
\hline Invasive ductal carcinoma & T2NOMO & 2 & Ila & malignant & +++ & ++ \\
\hline
\end{tabular}

$\begin{array}{cccc}+ & 129 & + & 4 \\ + & 132 & + & 4 \\ + & 141 & + & 4 \\ + & 167 & + & 4 \\ + & 67 & ++ & 4 \\ + & 79 & ++ & 4 \\ + & 81 & + & 4 \\ + & 113 & + & 4 \\ + & 109 & + & 4 \\ + & 86 & + & 4 \\ + & 82 & + & 4 \\ + & 67 & ++ & 4 \\ + & 75 & ++ & 4 \\ + & 77 & ++ & 4 \\ + & 81 & + & 4 \\ + & 82 & + & 4 \\ + & 92 & + & 4 \\ + & 98 & + & 4 \\ + & 87 & + & 4 \\ + & 103 & + & 4 \\ + & 109 & + & 4 \\ + & 121 & + & 4 \\ + & 118 & + & 4 \\ + & 105 & + & 4 \\ + & 109 & + & 4 \\ + & 176 & + & 4 \\ + & 182 & + & 4 \\ + & 189 & + & 4 \\ + & 98 & + & 4 \\ + & 112 & + & 4 \\ + & 106 & + & 4 \\ + & 89 & + & 4 \\ + & 162 & + & 4 \\ + & & & \end{array}$




$\begin{array}{llll}436 & 57 & \mathrm{~F} & \text { Breast } \\ 437 & 57 & \mathrm{~F} & \text { Breast } \\ 438 & 49 & \mathrm{~F} & \text { Breast } \\ 439 & 49 & \mathrm{~F} & \text { Breast } \\ 440 & 40 & \mathrm{~F} & \text { Breast } \\ 441 & 40 & \mathrm{~F} & \text { Breast } \\ 442 & 50 & \mathrm{~F} & \text { Breast } \\ 443 & 46 & \mathrm{~F} & \text { Breast } \\ 444 & 46 & \mathrm{~F} & \text { Breast } \\ 445 & 36 & \mathrm{~F} & \text { Breast } \\ 446 & 36 & \mathrm{~F} & \text { Breast } \\ 447 & 53 & \mathrm{~F} & \text { Breast } \\ 448 & 53 & \mathrm{~F} & \text { Breast } \\ 449 & 47 & \mathrm{~F} & \text { Breast } \\ 450 & 47 & \mathrm{~F} & \text { Breast } \\ 451 & 50 & \mathrm{~F} & \text { Breast } \\ 452 & 50 & \mathrm{~F} & \text { Breast } \\ 453 & 61 & \mathrm{~F} & \text { Breast } \\ 454 & 61 & \mathrm{~F} & \text { Breast } \\ 455 & 54 & \mathrm{~F} & \text { Breast } \\ 456 & 54 & \mathrm{~F} & \text { Breast } \\ 457 & 56 & \mathrm{~F} & \text { Breast } \\ 458 & 56 & \mathrm{~F} & \text { Breast } \\ 459 & 47 & \mathrm{~F} & \text { Breast } \\ 460 & 47 & \mathrm{~F} & \text { Breast } \\ 461 & 34 & \mathrm{~F} & \text { Breast } \\ 462 & 34 & \mathrm{~F} & \text { Breast } \\ 463 & 60 & \mathrm{~F} & \text { Breast } \\ 464 & 60 & \mathrm{~F} & \text { Breast } \\ 465 & 56 & \mathrm{~F} & \text { Breast } \\ 466 & 56 & \mathrm{~F} & \text { Breast } \\ 467 & 49 & \mathrm{~F} & \text { Breast } \\ 468 & 49 & \mathrm{~F} & \text { Breast } \\ 469 & 72 & \mathrm{~F} & \text { Breast } \\ & & & \end{array}$

\begin{tabular}{|c|c|c|c|c|c|c|}
\hline Invasive ductal carcinoma & T2N0M0 & 2 & Ila & malignant & - & - \\
\hline Invasive ductal carcinoma & T2NOMO & 2 & Ila & malignant & - & - \\
\hline Invasive ductal carcinoma & T2NOMO & 2 & Ila & malignant & + & - \\
\hline Invasive ductal carcinoma & T2NOMO & 2 & Ila & malignant & + & - \\
\hline Invasive ductal carcinoma & T2NOMO & 2 & Ila & malignant & - & - \\
\hline Invasive ductal carcinoma & T2NOMO & 2 & Ila & malignant & - & - \\
\hline Invasive ductal carcinoma & T2NOMO & 2 & Ila & malignant & + & + \\
\hline Invasive ductal carcinoma & T2NOMO & 2 & Ila & malignant & + & + \\
\hline Invasive ductal carcinoma & T2NOMO & 2 & Ila & malignant & + & + \\
\hline Invasive ductal carcinoma & T2NOMO & 2 & Ila & malignant & - & + \\
\hline Invasive ductal carcinoma & T2NOMO & 2 & Ila & malignant & - & + \\
\hline Invasive ductal carcinoma & T2NOMO & 2 & Ila & malignant & ++ & + \\
\hline Invasive ductal carcinoma & T2NOMO & 2 & Ila & malignant & ++ & + \\
\hline Invasive ductal carcinoma & T2NOMO & 2 & Ila & malignant & + & + \\
\hline Invasive ductal carcinoma & T2NOMO & 2 & Ila & malignant & + & + \\
\hline Invasive ductal carcinoma & T2NOMO & 2 & Ila & malignant & +++ & + \\
\hline Invasive ductal carcinoma & T2NOMO & 2 & Ila & malignant & +++ & + \\
\hline Invasive ductal carcinoma & T2N0M0 & 2 & Ila & malignant & + & + \\
\hline Invasive ductal carcinoma & T2NOMO & 2 & Ila & malignant & + & + \\
\hline Invasive ductal carcinoma & T2NOMO & 2 & Ila & malignant & + & + \\
\hline Invasive ductal carcinoma & T2NOMO & 2 & Ila & malignant & - & + \\
\hline Invasive ductal carcinoma & T2NOMO & 2 & Ila & malignant & +++ & + \\
\hline Invasive ductal carcinoma & T2NOMO & 2 & Ila & malignant & +++ & + \\
\hline Invasive ductal carcinoma & T2NOMO & 2 & Ila & malignant & +++ & + \\
\hline Invasive ductal carcinoma & T2NOMO & 2 & Ila & malignant & +++ & - \\
\hline Invasive ductal carcinoma & T2NOMO & 2 & Ila & malignant & ++ & - \\
\hline Invasive ductal carcinoma & T2NOMO & 2 & Ila & malignant & ++ & * \\
\hline Invasive ductal carcinoma & T2NOMO & 2 & Ila & malignant & +++ & - \\
\hline Invasive ductal carcinoma & T2NOMO & 2 & Ila & malignant & +++ & - \\
\hline Invasive ductal carcinoma & T2NOMO & 2 & Ila & malignant & + & - \\
\hline Invasive ductal carcinoma & T2NOMO & 2 & Ila & malignant & - & - \\
\hline Invasive ductal carcinoma & T2NOMO & 2 & Ila & malignant & - & - \\
\hline Invasive ductal carcinoma & T2NOMO & 2 & Ila & malignant & - & - \\
\hline Invasive ductal carcinoma & T2NOMO & 2 & Ila & malignant & ++ & - \\
\hline
\end{tabular}

$\begin{array}{cccc}+ & 176 & + & 4 \\ + & 106 & + & 4 \\ + & 101 & + & 4 \\ + & 78 & ++ & 4 \\ + & 85 & + & 4 \\ + & 87 & + & 4 \\ + & 89 & + & 4 \\ + & 82 & + & 4 \\ + & 89 & + & 4 \\ + & 106 & + & 4 \\ + & 87 & + & 4 \\ + & 79 & ++ & 4 \\ + & 109 & + & 4 \\ + & 103 & + & 4 \\ + & 86 & + & 4 \\ + & 84 & + & 4 \\ + & 129 & + & 4 \\ + & 134 & + & 4 \\ + & 145 & + & 4 \\ + & 113 & + & 4 \\ + & 87 & ++ & 4 \\ + & 134 & + & 4 \\ + & 128 & + & 4 \\ + & 131 & + & 4 \\ + & 129 & + & 4 \\ + & 89 & + & 4 \\ + & 103 & + & 4 \\ + & 109 & + & 4 \\ + & 118 & + & 4 \\ + & 131 & + & 4 \\ + & 98 & + & 4 \\ + & 83 & + & 4 \\ + & 153 & + & 4 \\ + & & & \end{array}$




\begin{tabular}{|c|c|c|c|c|c|c|c|c|c|c|}
\hline 470 & 72 & $\mathrm{~F}$ & Breast & Invasive ductal carcinoma & T2NOMO & 2 & Ila & malignant & ++ & - \\
\hline 471 & 57 & $\mathrm{~F}$ & Breast & nvasive ductal carcinoma (sparse & T2NOMO & 2 & Ila & malignant & ++ & * \\
\hline 472 & 57 & $\mathrm{~F}$ & Breast & Invasive ductal carcinoma & T2NOMO & 2 & Ila & malignant & ++ & * \\
\hline 473 & 41 & $\mathrm{~F}$ & Breast & Invasive ductal carcinoma & T2NOMO & 2 & Ila & malignant & ++ & - \\
\hline 474 & 41 & $\mathrm{~F}$ & Breast & Invasive ductal carcinoma & T2NOMO & 2 & Ila & malignant & ++ & - \\
\hline 475 & 58 & $\mathrm{~F}$ & Breast & Invasive ductal carcinoma & T2NOMO & 2 & Ila & malignant & +++ & - \\
\hline 476 & 58 & $\mathrm{~F}$ & Breast & Invasive ductal carcinoma & T2NOMO & 2 & Ila & malignant & +++ & - \\
\hline 477 & 40 & $\mathrm{~F}$ & Breast & Invasive ductal carcinoma & T2NOMO & 2 & Ila & malignant & ++ & - \\
\hline 478 & 40 & $\mathrm{~F}$ & Breast & Invasive ductal carcinoma & T2NOMO & 2 & Ila & malignant & ++ & - \\
\hline 479 & 61 & $\mathrm{~F}$ & Breast & Invasive ductal carcinoma & T2NOMO & 2 & Ila & malignant & + & - \\
\hline 480 & 61 & $\mathrm{~F}$ & Breast & Invasive ductal carcinoma & T2NOMO & 2 & Ila & malignant & + & - \\
\hline 481 & 64 & $\mathrm{~F}$ & Breast & Invasive ductal carcinoma & T2NOMO & 2 & Ila & malignant & - & - \\
\hline 482 & 64 & $\mathrm{~F}$ & Breast & Invasive ductal carcinoma & T2NOMO & 2 & Ila & malignant & - & * \\
\hline 483 & 68 & $\mathrm{~F}$ & Breast & Invasive ductal carcinoma & T2NOMO & 2 & Ila & malignant & - & * \\
\hline 484 & 68 & $\mathrm{~F}$ & Breast & Invasive ductal carcinoma & T2NOMO & 2 & Ila & malignant & - & * \\
\hline 485 & 50 & $\mathrm{~F}$ & Breast & Invasive ductal carcinoma & T2NOMO & 2 & Ila & malignant & - & - \\
\hline 486 & 50 & $\mathrm{~F}$ & Breast & Invasive ductal carcinoma & T2NOMO & 2 & Ila & malignant & - & - \\
\hline 487 & 56 & $\mathrm{~F}$ & Breast & Invasive ductal carcinoma & T2NOMO & 2 & Ila & malignant & - & * \\
\hline 488 & 56 & $\mathrm{~F}$ & Breast & Invasive ductal carcinoma & T2NOMO & 2 & Ila & ignant & - & * \\
\hline 489 & 48 & $\mathrm{~F}$ & Breast & Invasive ductal carcinoma & T2NOMO & 2 & Ila & lignant & +++ & - \\
\hline 490 & 48 & $\mathrm{~F}$ & Breast & Invasive ductal carcinoma & T2NOMO & 2 & Ila & lignant & +++ & - \\
\hline 491 & 57 & $\mathrm{~F}$ & Breast & Invasive ductal carcinoma & T2NOMO & 2 & Ila & malignant & +++ & - \\
\hline 492 & 57 & $\mathrm{~F}$ & Breast & Invasive ductal carcinoma & T2NOMO & 2 & Ila & malignant & +++ & - \\
\hline 493 & 49 & $\mathrm{~F}$ & Breast & Invasive ductal carcinoma & T2NOMO & 2 & Ila & malignant & - & * \\
\hline 494 & 49 & $\mathrm{~F}$ & Breast & Invasive ductal carcinoma & T2NOMO & 2 & Ila & malignant & - & * \\
\hline 495 & 53 & $\mathrm{~F}$ & Breast & sma with carcinomatous change o & T2NOMO & 2 & Ila & malignant & - & * \\
\hline 496 & 53 & $\mathrm{~F}$ & Breast & sma with carcinomatous change o & T2NOMO & 2 & Ila & malignant & - & * \\
\hline 497 & 34 & $\mathrm{~F}$ & Breast & Invasive ductal carcinoma & T2NOMO & 2 & Ila & malignant & +++ & +++ \\
\hline 498 & 34 & $\mathrm{~F}$ & Breast & Invasive ductal carcinoma & T2NOMO & 2 & Ila & malignant & +++ & +++ \\
\hline 499 & 61 & $\mathrm{~F}$ & Breast & Invasive ductal carcinoma & T2NOMO & 2 & Ila & malignant & +++ & +++ \\
\hline 500 & 50 & $\mathrm{~F}$ & Breast & nvasive ductal carcinoma (sparse & T2NOMO & 2 & Ila & malignant & ++ & ++ \\
\hline 501 & 56 & $\mathrm{~F}$ & Breast & Invasive ductal carcinoma & T2NOMO & 2 & Ila & malignant & - & - \\
\hline 502 & 56 & $\mathrm{~F}$ & Breast & Invasive ductal carcinoma & T2NOMO & 2 & Ila & malignant & - & - \\
\hline 503 & 45 & $\mathrm{~F}$ & Breast & Invasive ductal carcinoma & T2NOMO & 2 & Ila & malignant & + & ++ \\
\hline
\end{tabular}




\begin{tabular}{|c|c|c|c|c|c|c|c|c|c|c|}
\hline 504 & 45 & $\mathrm{~F}$ & Breast & Invasive ductal carcinoma & T2NOMO & 2 & Ila & malignant & + & ++ \\
\hline 505 & 41 & $\mathrm{~F}$ & Breast & Invasive ductal carcinoma & T2NOMO & 2 & Ila & malignant & * & ++ \\
\hline 506 & 41 & $\mathrm{~F}$ & Breast & Invasive ductal carcinoma & T2NOMO & 2 & Ila & malignant & * & * \\
\hline 507 & 43 & $\mathrm{~F}$ & Breast & Invasive ductal carcinoma & T2NOMO & 2 & Ila & malignant & +++ & +++ \\
\hline 508 & 43 & $\mathrm{~F}$ & Breast & Invasive ductal carcinoma & T2NOMO & 2 & Ila & malignant & +++ & +++ \\
\hline 09 & 51 & $\mathrm{~F}$ & Breast & Invasive ductal carcinoma & T2NOMO & 2 & Ila & malignant & ++ & + \\
\hline 10 & 51 & $\mathrm{~F}$ & Breast & Invasive ductal carcinoma & T2NOMO & 2 & Ila & malignant & ++ & + \\
\hline 11 & 55 & $\mathrm{~F}$ & Breast & nvasive ductal carcinoma (sparse & T2NOMO & 2 & Ila & malignant & - & - \\
\hline 12 & 55 & $\mathrm{~F}$ & Breast & Invasive ductal carcinoma & T2NOMO & 2 & Ila & malignant & - & - \\
\hline 13 & 46 & $\mathrm{~F}$ & Breast & Invasive ductal carcinoma & T2NOMO & 2 & Ila & malignant & +++ & ++ \\
\hline 14 & 46 & $\mathrm{~F}$ & Breast & Invasive ductal carcinoma & T2NOMO & 2 & Ila & malignant & +++ & ++ \\
\hline 15 & 46 & $\mathrm{~F}$ & Breast & Invasive ductal carcinoma & T2NOMO & 2 & Ila & malignant & +++ & +++ \\
\hline 16 & 46 & $\mathrm{~F}$ & Breast & Invasive ductal carcinoma & T2NOMO & 2 & Ila & malignant & +++ & +++ \\
\hline 17 & 41 & $\mathrm{~F}$ & Breast & nvasive ductal carcinoma (sparse & T2N0MO & 2 & Ila & malignant & ++ & ++ \\
\hline 18 & 41 & $\mathrm{~F}$ & Breast & Invasive ductal carcinoma & T2NOMO & 2 & Ila & ignant & ++ & ++ \\
\hline 19 & 45 & $\mathrm{~F}$ & Breast & Invasive ductal carcinoma & T2NOMO & 2 & Ila & lignant & - & - \\
\hline 20 & 45 & $\mathrm{~F}$ & Breast & Invasive ductal carcinoma & T2N0M0 & 2 & Ila & ignant & - & - \\
\hline 21 & 44 & $\mathrm{~F}$ & Breast & Invasive ductal carcinoma & T2N0M0 & 2 & Ila & alignant & +++ & +++ \\
\hline 522 & 44 & $\mathrm{~F}$ & Breast & Invasive ductal carcinoma & T2NOMO & 2 & Ila & malignant & +++ & +++ \\
\hline 523 & 44 & $\mathrm{~F}$ & Breast & asive ductal carcinoma with necrc & T2NOMO & 2 & Ila & malignant & +++ & +++ \\
\hline 524 & 44 & $\mathrm{~F}$ & Breast & Invasive ductal carcinoma & T2NOMO & 2 & Ila & malignant & +++ & +++ \\
\hline 525 & 45 & $\mathrm{~F}$ & Breast & Invasive ductal carcinoma & T2NOMO & 2 & Ila & malignant & +++ & +++ \\
\hline 526 & 45 & $\mathrm{~F}$ & Breast & Invasive ductal carcinoma & T2NOMO & 2 & Ila & malignant & +++ & +++ \\
\hline 527 & 44 & $\mathrm{~F}$ & Breast & asive ductal carcinoma with necrc & T2NOMO & 2 & Ila & malignant & +++ & ++ \\
\hline 528 & 44 & $\mathrm{~F}$ & Breast & Invasive ductal carcinoma & T2N0M0 & 2 & Ila & malignant & +++ & ++ \\
\hline 529 & 63 & $\mathrm{~F}$ & Breast & Invasive ductal carcinoma & T2NOMO & 2 & Ila & malignant & - & - \\
\hline 530 & 63 & $\mathrm{~F}$ & Breast & Invasive ductal carcinoma & T2N0M0 & 2 & Ila & malignant & - & - \\
\hline 531 & 70 & $\mathrm{~F}$ & Breast & Invasive ductal carcinoma & T2NOMO & 2 & Ila & malignant & +++ & - \\
\hline 532 & 70 & $\mathrm{~F}$ & Breast & Invasive ductal carcinoma & T2NOMO & 2 & Ila & malignant & +++ & - \\
\hline 533 & 47 & $\mathrm{~F}$ & Breast & Invasive ductal carcinoma & T2NOMO & 2 & Ila & malignant & - & - \\
\hline 534 & 47 & $\mathrm{~F}$ & Breast & Invasive ductal carcinoma & T2NOMO & 2 & Ila & malignant & - & - \\
\hline 535 & 57 & $\mathrm{~F}$ & Breast & Invasive ductal carcinoma & T2NOMO & 2 & Ila & malignant & + & - \\
\hline 536 & 57 & $\mathrm{~F}$ & Breast & Invasive ductal carcinoma & T2NOMO & 2 & Ila & malignant & + & - \\
\hline 537 & 47 & $\mathrm{~F}$ & Breast & Invasive ductal carcinoma & T2NOMO & 2 & Ila & malignant & - & - \\
\hline
\end{tabular}




\begin{tabular}{|c|c|c|c|c|c|c|c|c|c|c|}
\hline 538 & 47 & $\mathrm{~F}$ & Breast & Invasive ductal carcinoma & T2NOMO & 2 & Ila & malignant & - & - \\
\hline 539 & 55 & $\mathrm{~F}$ & Breast & Invasive ductal carcinoma & T2NOMO & 2 & Ila & malignant & + & - \\
\hline 540 & 55 & $\mathrm{~F}$ & Breast & Invasive ductal carcinoma & T2NOMO & 2 & Ila & malignant & + & - \\
\hline 541 & 57 & $\mathrm{~F}$ & Breast & asive ductal carcinoma with necrc & T2NOMO & 2 & Ila & malignant & - & - \\
\hline 542 & 55 & $\mathrm{~F}$ & Breast & Invasive ductal carcinoma & T2NOMO & 2 & Ila & malignant & +++ & ++ \\
\hline 543 & 55 & $\mathrm{~F}$ & Breast & Invasive ductal carcinoma & T2NOMO & 2 & Ila & malignant & +++ & ++ \\
\hline 544 & 49 & $\mathrm{~F}$ & Breast & Invasive ductal carcinoma & T2NOMO & 2 & Ila & malignant & +++ & +++ \\
\hline 45 & 49 & $\mathrm{~F}$ & Breast & Invasive ductal carcinoma & T2NOMO & 2 & Ila & malignant & +++ & +++ \\
\hline 546 & 36 & $\mathrm{~F}$ & Breast & Invasive ductal carcinoma & T2NOMO & 2 & Ila & malignant & - & - \\
\hline 47 & 36 & $\mathrm{~F}$ & Breast & Invasive ductal carcinoma & T2NOMO & 2 & Ila & malignant & - & - \\
\hline 548 & 47 & $\mathrm{~F}$ & Breast & Invasive ductal carcinoma & T2NOMO & 2 & Ila & malignant & +++ & +++ \\
\hline 549 & 47 & $\mathrm{~F}$ & Breast & Invasive ductal carcinoma & T2NOMO & 2 & Ila & malignant & +++ & +++ \\
\hline 550 & 72 & $\mathrm{~F}$ & Breast & Invasive ductal carcinoma & T2NOMO & 2 & Ila & malignant & +++ & - \\
\hline 551 & 72 & $\mathrm{~F}$ & Breast & Invasive ductal carcinoma & T2N0MO & 2 & Ila & malignant & +++ & - \\
\hline 552 & 57 & $\mathrm{~F}$ & Breast & Invasive ductal carcinoma & T2NOMO & 2 & Ila & ignant & - & - \\
\hline 553 & 57 & $\mathrm{~F}$ & Breast & Invasive ductal carcinoma & T2NOMO & 2 & Ila & malignant & - & - \\
\hline 554 & 51 & $\mathrm{~F}$ & Breast & Invasive ductal carcinoma & T2NOMO & 2 & Ila & ignant & - & - \\
\hline 555 & 51 & $\mathrm{~F}$ & Breast & Invasive ductal carcinoma & T2NOMO & 2 & Ila & malignant & - & - \\
\hline 556 & 52 & $\mathrm{~F}$ & Breast & Invasive ductal carcinoma & T2NOMO & 2 & Ila & malignant & ++ & + \\
\hline 557 & 52 & $\mathrm{~F}$ & Breast & Invasive ductal carcinoma & T2NOMO & 2 & Ila & malignant & ++ & + \\
\hline 558 & 48 & $\mathrm{~F}$ & Breast & Invasive ductal carcinoma & T2NOMO & 2 & Ila & malignant & - & - \\
\hline 559 & 48 & $\mathrm{~F}$ & Breast & Invasive ductal carcinoma & T2NOMO & 2 & Ila & malignant & - & - \\
\hline 560 & 60 & $\mathrm{~F}$ & Breast & Invasive ductal carcinoma & T2NOMO & 2 & Ila & malignant & - & - \\
\hline 561 & 60 & $\mathrm{~F}$ & Breast & Invasive ductal carcinoma & T2NOMO & 2 & Ila & malignant & - & - \\
\hline 562 & 36 & $\mathrm{~F}$ & Breast & Invasive ductal carcinoma & T2N0M0 & 2 & Ila & malignant & +++ & + \\
\hline 563 & 36 & $\mathrm{~F}$ & Breast & Invasive ductal carcinoma & T2NOMO & 2 & Ila & malignant & +++ & + \\
\hline 564 & 33 & $\mathrm{~F}$ & Breast & Invasive ductal carcinoma & T2N0M0 & 2 & Ila & malignant & +++ & + \\
\hline 565 & 33 & $\mathrm{~F}$ & Breast & Invasive ductal carcinoma & T2NOMO & 2 & Ila & malignant & +++ & + \\
\hline 566 & 52 & $\mathrm{~F}$ & Breast & Invasive ductal carcinoma & T2NOMO & 2 & Ila & malignant & - & + \\
\hline 567 & 66 & $\mathrm{~F}$ & Breast & Invasive ductal carcinoma & T2NOMO & 2 & Ila & malignant & - & - \\
\hline 568 & 66 & $\mathrm{~F}$ & Breast & Invasive ductal carcinoma & T2NOMO & 2 & Ila & malignant & - & - \\
\hline 569 & 64 & $\mathrm{~F}$ & Breast & Invasive ductal carcinoma & T2NOMO & 2 & Ila & malignant & - & - \\
\hline 570 & 64 & $\mathrm{~F}$ & Breast & Invasive ductal carcinoma & T2NOMO & 2 & Ila & malignant & - & - \\
\hline 571 & 35 & $\mathrm{~F}$ & Breast & asive ductal carcinoma with necrc & T2NOMO & 2 & Ila & malignant & - & - \\
\hline
\end{tabular}


Breast asive ductal carcinoma with necrc T2NOMO Invasive ductal carcinoma T2NOMO

\section{2}

\section{Breast}

Breast

Breast

Invasive ductal carcinoma

T2NOMO

Invasive ductal carcinoma

T2NOMO

Invasive ductal carcinoma

T2NOMO

Invasive ductal carcinoma

T2NOMO

Breast

Invasive ductal carcinoma

T2NOMO

Invasive ductal carcinoma

T2NOMO

Invasive ductal carcinoma

T2NOMO

Invasive ductal carcinoma

T2NOMO

Breast

Breast

nvasive ductal carcinoma (sparse

$\mathrm{T} 2 \mathrm{~N} 0 \mathrm{MO}$

T2NOMO

Breast

asive ductal carcinoma with necrc T2NOMO

Invasive ductal carcinoma T2NOMO

Invasive ductal carcinoma T2NOMO

Breast

Breast

asive ductal carcinoma with necrc T2NOMO

Invasive ductal carcinoma T2NOMO

Breast

nvasive ductal carcinoma (sparse T2NOMO

Breast asive ductal carcinoma with necrc T2NOMO

Breast $ə$ ductal carcinoma (sparse) with $r$ T2NOMO

Breast vasive ductal carcinoma (sparse T2NOMO

T2NOMO

Breast

Invasive ductal carcinoma

T2NOMO

Invasive ductal carcinoma

T2NOMO

Breast

Breast

Breast

vasive ductal carcinom

T2NOMO

Invasive ductal carcinoma

T2NOMO

Invasive ductal carcinoma

T2NOMO

Invasive ductal carcinoma

T2NOMO

Invasive ductal carcinoma

T2NOMO

T2NOMO

Invasive ductal carcinoma

T2NOMO

Invasive ductal carcinoma

$\mathrm{T} 2 \mathrm{NOMO}$

Breast

Breast

Breast

nvasive ductal carcinoma (sparse T2NOMO

Invasive ductal carcinoma

T2NOMO

$\begin{array}{lcccc}\text { Ila } & \text { malignant } & - & - & 0 \\ \text { Ila } & \text { malignant } & +++ & - & 0 \\ \text { Ila } & \text { malignant } & +++ & - & 0 \\ \text { Ila } & \text { malignant } & +++ & ++ & 3+ \\ \text { Ila } & \text { malignant } & +++ & ++ & 3+ \\ \text { Ila } & \text { malignant } & +++ & ++ & 0 \\ \text { Ila } & \text { malignant } & +++ & ++ & 0 \\ \text { Ila } & \text { malignant } & +++ & + & 0 \\ \text { Ila } & \text { malignant } & ++ & - & 3+ \\ \text { Ila } & \text { malignant } & ++ & - & 3+ \\ \text { Ila } & \text { malignant } & - & - & 0 \\ \text { Ila } & \text { malignant } & - & - & 0 \\ \text { Ila } & \text { malignant } & - & - & 0 \\ \text { Ila } & \text { malignant } & - & - & 0 \\ \text { Ila } & \text { malignant } & + & - & 0 \\ \text { Ila } & \text { malignant } & + & - & 0 \\ \text { Ila } & \text { malignant } & +++ & - & 0 \\ \text { Ila } & \text { malignant } & +++ & - & 0 \\ \text { Ila } & \text { malignant } & - & - & 0 \\ \text { Ila } & \text { malignant } & - & - & 0 \\ \text { Ila } & \text { malignant } & ++ & - & 0 \\ \text { Ila } & \text { malignant } & ++ & - & 0 \\ \text { Ila } & \text { malignant } & - & - & 0 \\ \text { Ila } & \text { malignant } & ++ & - & 0 \\ \text { Ila } & \text { malignant } & ++ & - & 0 \\ \text { Ila } & \text { malignant } & ++ & - & 0 \\ \text { Ila } & \text { malignant } & ++ & - & 0 \\ \text { Ila } & \text { malignant } & - & - & 1+ \\ \text { Ila } & \text { malignant } & - & - & 1+ \\ & \text { malignant } & +++ & - & 0 \\ \text { Ilignant } & +++ & - & 0 \\ \text { Ilignant } & - & - & 3+ \\ \text { Il } & - & - & 3+ \\ \text { Il } & - & - & 0\end{array}$

$\begin{array}{cccc}+ & 158 & + & 4 \\ + & 99 & + & 4 \\ + & 102 & + & 4 \\ + & 86 & + & 4 \\ + & 120 & + & 4 \\ + & 93 & + & 4 \\ + & 92 & + & 4 \\ + & 134 & + & 4 \\ + & 142 & + & 4 \\ + & 89 & ++ & 4 \\ + & 68 & ++ & 4 \\ + & 73 & ++ & 4 \\ + & 78 & ++ & 4 \\ + & 82 & + & 4 \\ + & 89 & + & 4 \\ + & 56 & + & 4 \\ + & 102 & + & 4 \\ + & 104 & + & 4 \\ + & 126 & + & 4 \\ + & 112 & + & 4 \\ + & 98 & + & 4 \\ + & 87 & + & 4 \\ + & 86 & + & 4 \\ + & 93 & + & 4 \\ + & 95 & + & 4 \\ + & 107 & + & 4 \\ + & 102 & + & 4 \\ + & 87 & + & 4 \\ + & 116 & + & 4 \\ + & 98 & + & 4 \\ + & 93 & + & 4 \\ + & 103 & + & 4 \\ + & 96 & + & 4 \\ + & & & \end{array}$




\begin{tabular}{|c|c|c|c|c|c|c|c|c|c|c|}
\hline 606 & 46 & $\mathrm{~F}$ & Breast & Invasive ductal carcinoma & T2NOMO & 2 & Ila & malignant & - & - \\
\hline 607 & 48 & $\mathrm{~F}$ & Breast & Invasive ductal carcinoma & T2NOMO & 2 & Ila & malignant & + & + \\
\hline 608 & 48 & $\mathrm{~F}$ & Breast & Invasive ductal carcinoma & T2NOMO & 2 & Ila & malignant & + & + \\
\hline 609 & 51 & $\mathrm{~F}$ & Breast & asive ductal carcinoma with necrc & T2NOMO & 2 & Ila & malignant & - & - \\
\hline 610 & 51 & $\mathrm{~F}$ & Breast & asive ductal carcinoma with necrc & T2NOMO & 2 & Ila & malignant & - & - \\
\hline 611 & 42 & $\mathrm{~F}$ & Breast & Invasive ductal carcinoma & T2NOMO & 2 & Ila & malignant & ++ & - \\
\hline 612 & 42 & $\mathrm{~F}$ & Breast & Invasive ductal carcinoma & T2NOMO & 2 & Ila & malignant & ++ & - \\
\hline 613 & 65 & $\mathrm{~F}$ & Breast & Invasive ductal carcinoma & T2N0M0 & 2 & Ila & malignant & +++ & ++ \\
\hline 14 & 65 & $\mathrm{~F}$ & Breast & Invasive ductal carcinoma & T2NOMO & 2 & Ila & malignant & +++ & ++ \\
\hline 15 & 48 & $\mathrm{~F}$ & Breast & Invasive ductal carcinoma & T2NOMO & 2 & Ila & malignant & + & - \\
\hline 16 & 56 & $\mathrm{~F}$ & Breast & Invasive ductal carcinoma & T2NOMO & 2 & Ila & malignant & ++ & - \\
\hline 617 & 56 & $\mathrm{~F}$ & Breast & Invasive ductal carcinoma & T2NOMO & 2 & Ila & malignant & ++ & - \\
\hline 618 & 47 & $\mathrm{~F}$ & Breast & Invasive ductal carcinoma & T2NOMO & 2 & Ila & malignant & - & + \\
\hline 619 & 47 & $\mathrm{~F}$ & Breast & Invasive ductal carcinoma & T2NOMO & 2 & Ila & malignant & * & + \\
\hline 620 & 57 & $\mathrm{~F}$ & Breast & Invasive ductal carcinoma & T2NOMO & 2 & Ila & malignant & - & - \\
\hline 621 & 57 & $\mathrm{~F}$ & Breast & nvasive ductal carcinoma (sparse & T2NOMO & 2 & Ila & malignant & - & - \\
\hline 622 & 40 & $\mathrm{~F}$ & Breast & Invasive ductal carcinoma & T2NOMO & 2 & Ila & malignant & +++ & - \\
\hline 623 & 64 & $\mathrm{~F}$ & Breast & nvasive ductal carcinoma (sparse & T2NOMO & 2 & Ila & malignant & ++ & + \\
\hline 624 & 49 & $\mathrm{~F}$ & Breast & Invasive ductal carcinoma & T2NOMO & 2 & Ila & malignant & - & - \\
\hline 625 & 49 & $\mathrm{~F}$ & Breast & Invasive ductal carcinoma & T2NOMO & 2 & Ila & malignant & - & - \\
\hline 626 & 43 & $\mathrm{~F}$ & Breast & Invasive ductal carcinoma & T2NOMO & 2 & Ila & malignant & +++ & ++ \\
\hline 627 & 43 & $\mathrm{~F}$ & Breast & Invasive ductal carcinoma & T2NOMO & 2 & Ila & malignant & +++ & ++ \\
\hline 628 & 72 & $\mathrm{~F}$ & Breast & Invasive ductal carcinoma & T2NOMO & 2 & Ila & malignant & +++ & +++ \\
\hline 629 & 72 & $\mathrm{~F}$ & Breast & Invasive ductal carcinoma & T2NOMO & 2 & Ila & malignant & +++ & +++ \\
\hline 630 & 36 & $\mathrm{~F}$ & Breast & Invasive ductal carcinoma & T2NOMO & 2 & Ila & malignant & + & + \\
\hline 631 & 36 & $\mathrm{~F}$ & Breast & Invasive ductal carcinoma & T2NOMO & 2 & Ila & malignant & + & + \\
\hline 632 & 48 & $\mathrm{~F}$ & Breast & Invasive ductal carcinoma & T2NOMO & 2 & Ila & malignant & - & + \\
\hline 633 & 48 & $\mathrm{~F}$ & Breast & Invasive ductal carcinoma & T2N0MO & 2 & Ila & malignant & - & + \\
\hline 634 & 40 & $\mathrm{~F}$ & Breast & nvasive ductal carcinoma (sparse & T2N0M0 & 2 & Ila & malignant & * & * \\
\hline 635 & 57 & $\mathrm{~F}$ & Breast & Invasive ductal carcinoma & T2NOMO & 2 & Ila & malignant & - & - \\
\hline 636 & 57 & $\mathrm{~F}$ & Breast & Invasive ductal carcinoma & T2NOMO & 2 & Ila & malignant & - & - \\
\hline 637 & 51 & $\mathrm{~F}$ & Breast & Invasive ductal carcinoma & T2NOMO & 2 & Ila & malignant & - & - \\
\hline 638 & 51 & $\mathrm{~F}$ & Breast & Invasive ductal carcinoma & T2NOMO & 2 & Ila & malignant & - & - \\
\hline 639 & 45 & $\mathrm{~F}$ & Breast & Invasive ductal carcinoma & T2N0M0 & 2 & Ila & malignant & - & + \\
\hline
\end{tabular}




$\begin{array}{llll}640 & 45 & \mathrm{~F} & \text { Breast } \\ 641 & 42 & \mathrm{~F} & \text { Breast } \\ 642 & 42 & \mathrm{~F} & \text { Breast } \\ 643 & 41 & \mathrm{~F} & \text { Breast } \\ 644 & 41 & \mathrm{~F} & \text { Breast } \\ 645 & 67 & \mathrm{~F} & \text { Breast } \\ 646 & 67 & \mathrm{~F} & \text { Breast } \\ 647 & 38 & \mathrm{~F} & \text { Breast } \\ 648 & 38 & \mathrm{~F} & \text { Breast } \\ 649 & 48 & \mathrm{~F} & \text { Breast } \\ 650 & 48 & \mathrm{~F} & \text { Breast } \\ 651 & 57 & \mathrm{~F} & \text { Breast } \\ 652 & 57 & \mathrm{~F} & \text { Breast } \\ 653 & 56 & \mathrm{~F} & \text { Breast } \\ 654 & 56 & \mathrm{~F} & \text { Breast } \\ 655 & 45 & \mathrm{~F} & \text { Breast } \\ 656 & 45 & \mathrm{~F} & \text { Breast } \\ 657 & 55 & \mathrm{~F} & \text { Breast } \\ 658 & 55 & \mathrm{~F} & \text { Breast } \\ 659 & 49 & \mathrm{~F} & \text { Breast } \\ 660 & 49 & \mathrm{~F} & \text { Breast } \\ 661 & 36 & \mathrm{~F} & \text { Breast } \\ 662 & 36 & \mathrm{~F} & \text { Breast } \\ 663 & 42 & \mathrm{~F} & \text { Breast } \\ 664 & 42 & \mathrm{~F} & \text { Breast } \\ 665 & 49 & \mathrm{~F} & \text { Breast } \\ 666 & 49 & \mathrm{~F} & \text { Breast } \\ 667 & 56 & \mathrm{~F} & \text { Breast } \\ 668 & 56 & \mathrm{~F} & \text { Breast } \\ 669 & 69 & \mathrm{~F} & \text { Breast } \\ 670 & 47 & \mathrm{~F} & \text { Breast } \\ 671 & 47 & \mathrm{~F} & \text { Breast } \\ 672 & 52 & \mathrm{~F} & \text { Breast } \\ 673 & 38 & \mathrm{~F} & \text { Breast } \\ & & & \end{array}$

\begin{tabular}{|c|c|c|c|c|c|c|}
\hline Invasive ductal carcinoma & T2N0MO & 2 & Ila & malignant & - & + \\
\hline Invasive ductal carcinoma & T2NOMO & 2 & Ila & malignant & +++ & + \\
\hline Invasive ductal carcinoma & T2NOMO & 2 & Ila & malignant & +++ & + \\
\hline Invasive ductal carcinoma & T2N0MO & 2 & Ila & malignant & ++ & ++ \\
\hline Invasive ductal carcinoma & T2NOMO & 2 & Ila & malignant & ++ & ++ \\
\hline Invasive ductal carcinoma & T2NOMO & 2 & Ila & malignant & ++ & - \\
\hline Invasive ductal carcinoma & T2NOMO & 2 & Ila & malignant & ++ & - \\
\hline Invasive ductal carcinoma & T2NOMO & 2 & Ila & malignant & - & - \\
\hline Invasive ductal carcinoma & T2NOMO & 2 & Ila & malignant & - & - \\
\hline Invasive ductal carcinoma & T2NOMO & 2 & Ila & malignant & ++ & ++ \\
\hline Invasive ductal carcinoma & T2NOMO & 2 & Ila & malignant & ++ & ++ \\
\hline Invasive ductal carcinoma & T2NOMO & 2 & Ila & malignant & - & - \\
\hline Invasive ductal carcinoma & T2NOMO & 2 & Ila & malignant & - & - \\
\hline Invasive ductal carcinoma & T2NOMO & 2 & Ila & malignant & ++ & + \\
\hline Invasive ductal carcinoma & T2NOMO & 2 & Ila & malignant & ++ & + \\
\hline Invasive ductal carcinoma & T2NOMO & 2 & Ila & malignant & - & - \\
\hline Invasive ductal carcinoma & T2NOMO & 2 & Ila & malignant & - & + \\
\hline Invasive ductal carcinoma & T2NOMO & 2 & Ila & malignant & - & - \\
\hline Invasive ductal carcinoma & T2NOMO & 2 & Ila & malignant & - & - \\
\hline Invasive ductal carcinoma & T2NOMO & 2 & Ila & malignant & +++ & ++ \\
\hline Invasive ductal carcinoma & T2NOMO & 2 & Ila & & +++ & ++ \\
\hline Invasive ductal carcinoma & T2NOMO & 2 & Ila & malignant & +++ & ++ \\
\hline Invasive ductal carcinoma & T2NOMO & 2 & Ila & malignant & +++ & ++ \\
\hline Invasive ductal carcinoma & T2NOMO & 2 & Ila & malignant & +++ & +++ \\
\hline Invasive ductal carcinoma & T2NOMO & 2 & Ila & malignant & +++ & +++ \\
\hline Invasive ductal carcinoma & T2NOMO & 2 & Ila & malignant & +++ & +++ \\
\hline Invasive ductal carcinoma & T2NOMO & 2 & Ila & malignant & +++ & +++ \\
\hline Invasive ductal carcinoma & T2NOMO & 2 & Ila & malignant & +++ & +++ \\
\hline Invasive ductal carcinoma & T2NOMO & 2 & Ila & malignant & +++ & +++ \\
\hline Invasive ductal carcinoma & T2NOMO & 2 & Ila & malignant & +++ & + \\
\hline Invasive ductal carcinoma & T2NOMO & 2 & Ila & malignant & - & - \\
\hline Invasive ductal carcinoma & T2NOMO & 2 & Ila & malignant & - & - \\
\hline Invasive ductal carcinoma & T2NOMO & 2 & Ila & malignant & - & - \\
\hline Invasive ductal carcinoma & T2NOMO & 2 & Ila & malignant & - & - \\
\hline
\end{tabular}

$\begin{array}{cccc}+ & 72 & ++ & 4 \\ + & 63 & ++ & 4 \\ + & 79 & + & 4 \\ + & 92 & + & 4 \\ + & 75 & + & 4 \\ + & 67 & ++ & 4 \\ + & 65 & ++ & 4 \\ + & 54 & ++ & 4 \\ + & 68 & ++ & 4 \\ + & 62 & ++ & 4 \\ + & 71 & + & 4 \\ + & 83 & + & 4 \\ + & 93 & + & 4 \\ + & 81 & + & 4 \\ + & 107 & + & 4 \\ + & 122 & + & 4 \\ + & 103 & + & 4 \\ + & 87 & + & 4 \\ + & 83 & + & 4 \\ + & 112 & + & 4 \\ + & 87 & + & 4 \\ + & 65 & + & 4 \\ + & 76 & + & 4 \\ + & 79 & ++ & 4 \\ + & 86 & + & 4 \\ + & 107 & + & 4 \\ + & 129 & + & 4 \\ + & 67 & ++ & 4 \\ + & 75 & ++ & 4 \\ + & 84 & + & 4 \\ + & 92 & + & 4 \\ + & 76 & ++ & 4 \\ + & 102 & + & 4 \\ + & 119 & + & 4 \\ + & & & \end{array}$




\begin{tabular}{|c|c|c|c|c|c|c|c|c|c|c|}
\hline 674 & 38 & $\mathrm{~F}$ & Breast & nvasive ductal carcinoma (sparse & T2NOMO & 2 & Ila & malignant & - & - \\
\hline 675 & 76 & $\mathrm{~F}$ & Breast & Invasive ductal carcinoma & T2NOMO & 2 & Ila & malignant & +++ & + \\
\hline 676 & 76 & $\mathrm{~F}$ & Breast & Invasive ductal carcinoma & T2NOMO & 2 & Ila & malignant & +++ & + \\
\hline 677 & 33 & $\mathrm{~F}$ & Breast & Invasive ductal carcinoma & T2NOMO & 2 & Ila & malignant & - & - \\
\hline 678 & 33 & $\mathrm{~F}$ & Breast & Invasive ductal carcinoma & T2NOMO & 2 & Ila & malignant & - & - \\
\hline 679 & 46 & $\mathrm{~F}$ & Breast & Invasive ductal carcinoma & T2NOMO & 2 & Ila & malignant & ++ & + \\
\hline 680 & 46 & $\mathrm{~F}$ & Breast & ə ductal carcinoma (sparse) with $r$ & T2NOMO & 2 & Ila & malignant & + & - \\
\hline 681 & 42 & $\mathrm{~F}$ & Breast & Invasive ductal carcinoma & T2NOMO & 2 & Ila & malignant & - & - \\
\hline 682 & 42 & $\mathrm{~F}$ & Breast & Invasive ductal carcinoma & T2NOMO & 2 & Ila & malignant & - & - \\
\hline 683 & 67 & $\mathrm{~F}$ & Breast & Invasive ductal carcinoma & T2NOMO & 2 & Ila & malignant & +++ & + \\
\hline 684 & 67 & $\mathrm{~F}$ & Breast & Invasive ductal carcinoma & T2NOMO & 2 & Ila & malignant & +++ & + \\
\hline 685 & 50 & $\mathrm{~F}$ & Breast & Invasive ductal carcinoma & T2N0MO & 2 & Ila & malignant & - & - \\
\hline 686 & 50 & $\mathrm{~F}$ & Breast & Invasive ductal carcinoma & T2NOMO & 2 & Ila & malignant & - & - \\
\hline 687 & 43 & $\mathrm{~F}$ & Breast & Invasive ductal carcinoma & T2NOMO & 2 & Ila & malignant & + & + \\
\hline 688 & 43 & $\mathrm{~F}$ & Breast & Invasive ductal carcinoma & T2NOMO & 2 & Ila & malignant & + & + \\
\hline 689 & 32 & $\mathrm{~F}$ & Breast & Invasive ductal carcinoma & T2NOMO & 2 & Ila & malignant & - & - \\
\hline 690 & 32 & $\mathrm{~F}$ & Breast & Invasive ductal carcinoma & T2NOMO & 2 & Ila & malignant & - & - \\
\hline 691 & 50 & $\mathrm{~F}$ & Breast & Invasive ductal carcinoma & T2NOMO & 2 & Ila & malignant & +++ & +++ \\
\hline 692 & 50 & $\mathrm{~F}$ & Breast & Invasive ductal carcinoma & T2NOMO & 2 & Ila & malignant & +++ & +++ \\
\hline 693 & 52 & $\mathrm{~F}$ & Breast & Invasive ductal carcinoma & T2NOMO & 2 & Ila & lignant & +++ & +++ \\
\hline 694 & 52 & $\mathrm{~F}$ & Breast & Invasive ductal carcinoma & T2NOMO & 2 & Ila & malignant & +++ & +++ \\
\hline 695 & 60 & $\mathrm{~F}$ & Breast & nvasive ductal carcinoma (sparse & T2NOMO & 2 & Ila & malignant & ++ & - \\
\hline 696 & 60 & $\mathrm{~F}$ & Breast & Invasive ductal carcinoma & T2NOMO & 2 & Ila & malignant & ++ & - \\
\hline 697 & 46 & $\mathrm{~F}$ & Breast & Invasive ductal carcinoma & T2NOMO & 2 & Ila & malignant & + & - \\
\hline 698 & 40 & $\mathrm{~F}$ & Breast & Invasive ductal carcinoma & T2NOMO & 2 & Ila & malignant & +++ & +++ \\
\hline 699 & 40 & $\mathrm{~F}$ & Breast & Invasive ductal carcinoma & T2NOMO & 2 & Ila & malignant & +++ & +++ \\
\hline 700 & 45 & $\mathrm{~F}$ & Breast & Invasive ductal carcinoma & T2NOMO & 2 & Ila & malignant & +++ & ++ \\
\hline 701 & 45 & $\mathrm{~F}$ & Breast & Invasive ductal carcinoma & T2NOMO & 2 & Ila & malignant & +++ & + \\
\hline 702 & 50 & $\mathrm{~F}$ & Breast & Invasive ductal carcinoma & T2NOMO & 2 & Ila & malignant & +++ & + \\
\hline 703 & 50 & $\mathrm{~F}$ & Breast & Invasive ductal carcinoma & T2NOMO & 2 & Ila & malignant & +++ & + \\
\hline 704 & 51 & $\mathrm{~F}$ & Breast & Invasive ductal carcinoma & T2NOMO & 2 & Ila & malignant & ++ & +++ \\
\hline 705 & 51 & $\mathrm{~F}$ & Breast & Invasive ductal carcinoma & T2NOMO & 2 & Ila & malignant & ++ & +++ \\
\hline 706 & 38 & $\mathrm{~F}$ & Breast & Invasive ductal carcinoma & T2NOMO & 2 & Ila & malignant & ++ & + \\
\hline 707 & 38 & $\mathrm{~F}$ & Breast & Invasive ductal carcinoma & T2N0MO & 2 & Ila & malignant & ++ & + \\
\hline
\end{tabular}




$\begin{array}{llll}708 & 51 & \mathrm{~F} & \text { Breast } \\ 709 & 51 & \mathrm{~F} & \text { Breast } \\ 710 & 55 & \mathrm{~F} & \text { Breast } \\ 711 & 55 & \mathrm{~F} & \text { Breast } \\ 712 & 55 & \mathrm{~F} & \text { Breast } \\ 713 & 55 & \mathrm{~F} & \text { Breast } \\ 714 & 40 & \mathrm{~F} & \text { Breast } \\ 715 & 40 & \mathrm{~F} & \text { Breast } \\ 716 & 49 & \mathrm{~F} & \text { Breast } \\ 717 & 55 & \mathrm{~F} & \text { Breast } \\ 718 & 55 & \mathrm{~F} & \text { Breast } \\ 719 & 56 & \mathrm{~F} & \text { Breast } \\ 720 & 56 & \mathrm{~F} & \text { Breast } \\ 721 & 56 & \mathrm{~F} & \text { Breast } \\ 722 & 56 & \mathrm{~F} & \text { Breast } \\ 723 & 46 & \mathrm{~F} & \text { Breast } \\ 724 & 46 & \mathrm{~F} & \text { Breast } \\ 725 & 40 & \mathrm{~F} & \text { Breast } \\ 726 & 40 & \mathrm{~F} & \text { Breast } \\ 727 & 50 & \mathrm{~F} & \text { Breast } \\ 728 & 50 & \mathrm{~F} & \text { Breast } \\ 729 & 56 & \mathrm{~F} & \text { Breast } \\ 730 & 56 & \mathrm{~F} & \text { Breast } \\ 731 & 61 & \mathrm{~F} & \text { Breast } \\ 732 & 61 & \mathrm{~F} & \text { Breast } \\ 733 & 71 & \mathrm{~F} & \text { Breast } \\ 734 & 48 & \mathrm{~F} & \text { Breast } \\ 735 & 48 & \mathrm{~F} & \text { Breast } \\ 736 & 48 & \mathrm{~F} & \text { Breast } \\ 738 & 48 & \mathrm{~F} & \text { Breast } \\ 739 & 49 & \mathrm{~F} & \text { Breast } \\ 740 & 56 & \mathrm{~F} & \text { Breast } \\ 741 & 56 & \mathrm{~F} & \text { Breast } \\ & & & \end{array}$

Invasive ductal carcinoma

Invasive ductal carcinoma

Invasive ductal carcinoma

Invasive ductal carcinoma

Invasive ductal carcinoma

nvasive ductal carcinoma (sparse
Invasive ductal carcinoma

Invasive ductal carcinoma

Invasive ductal carcinoma

Invasive ductal carcinoma

Invasive ductal carcinoma

Invasive ductal carcinoma

Invasive ductal carcinoma

Papillary carcinoma

Papillary carcinoma

Adenocarcinoma

Adenocarcinoma

Adenocarcinoma

Adenocarcinoma

Invasive ductal carcinoma

Invasive ductal carcinoma

Invasive ductal carcinoma

Invasive ductal carcinoma

Invasive ductal carcinoma

Invasive ductal carcinoma

Invasive ductal carcinoma

Invasive ductal carcinoma

Invasive ductal carcinoma

Invasive ductal carcinoma

Invasive ductal carcinoma

Invasive ductal carcinoma

Invasive ductal carcinoma

Invasive ductal carcinoma

Invasive ductal carcinoma

$\begin{array}{llllccc}\text { T2NOM0 } & 2 & \text { Ila } & \text { malignant } & + & - & 3+ \\ \text { T2NOM0 } & 2 & \text { Ila } & \text { malignant } & + & - & 3+ \\ \text { T2NOM0 } & 2 & \text { Ila } & \text { malignant } & +++ & ++ & 0 \\ \text { T2NOM0 } & 2 & \text { Ila } & \text { malignant } & +++ & ++ & 0 \\ \text { T2NOM0 } & 2 & \text { Ila } & \text { malignant } & +++ & - & 0 \\ \text { T2NOM0 } & 2 & \text { Ila } & \text { malignant } & +++ & - & 0 \\ \text { T2NOM0 } & 2 & \text { Ila } & \text { malignant } & - & - & 0 \\ \text { T2NOM0 } & 2 & \text { Ila } & \text { malignant } & - & - & 0 \\ \text { T2NOM0 } & 2 & \text { Ila } & \text { malignant } & + & - & 0 \\ \text { T2NOM0 } & 2 & \text { Ila } & \text { malignant } & - & - & 2+ \\ \text { T2NOM0 } & 2 & \text { Ila } & \text { malignant } & - & - & * \\ \text { T2NOM0 } & 2 & \text { Ila } & \text { malignant } & - & - & 1+ \\ \text { T2NOM0 } & 2 & \text { Ila } & \text { malignant } & - & - & 1+ \\ \text { T2NOM0 } & 2 & \text { Ila } & \text { malignant } & ++ & - & 0 \\ \text { T2NOM0 } & 2 & \text { Ila } & \text { malignant } & ++ & - & 0 \\ \text { T2NOM0 } & 2 & \text { Ila } & \text { malignant } & + & - & 0 \\ \text { T2NOM0 } & 2 & \text { Ila } & \text { malignant } & + & - & 0 \\ \text { T2NOM0 } & 2 & \text { Ila } & \text { malignant } & +++ & - & 0 \\ \text { T2NOM0 } & 2 & \text { Ila } & \text { malignant } & +++ & - & 0 \\ \text { T2NOM0 } & 2 & \text { Ila } & \text { malignant } & - & * & 0 \\ \text { T2NOM0 } & 2 & \text { Ila } & \text { malignant } & - & * & 0 \\ \text { T2NOM0 } & 2 & \text { Ila } & \text { malignant } & ++ & - & 0 \\ \text { T2NOM0 } & 2 & \text { Ila } & \text { malignant } & ++ & - & 0 \\ \text { T2NOM0 } & 2 & \text { Ila } & \text { malignant } & - & * & 2+ \\ \text { T2NOM0 } & 2 & \text { Ila } & \text { malignant } & - & * & 2+ \\ \text { T2NOM0 } & 2 & \text { Ila } & \text { malignant } & +++ & * & 0 \\ \text { T2NOM0 } & 2 & \text { Ila } & \text { malignant } & - & - & 0 \\ \text { T2NOM0 } & 2 & \text { Ila } & \text { malignant } & - & - & 0 \\ \text { T2NOM0 } & 2 & \text { Ila } & \text { malignant } & - & - & 0 \\ \text { T2NOM0 } & 2 & \text { Ila } & \text { malignant } & - & - & 0 \\ \text { T2NOM0 } & 2 & \text { Ila } & \text { malignant } & - & * & 3+ \\ \text { T2NOM0 } & 2 & \text { Ila } & \text { malignant } & - & * & 3+ \\ \text { T2NOM0 } & 2 & \text { Ila } & \text { malignant } & - & - & 0 \\ \text { T2NOM0 } & 2 & \text { Ila } & \text { malignant } & - & - & 0\end{array}$

$\begin{array}{cccc}+ & 67 & ++ & 4 \\ + & 82 & + & 4 \\ + & 71 & ++ & 4 \\ + & 103 & + & 4 \\ + & 127 & + & 4 \\ + & 82 & + & 4 \\ + & 66 & + & 4 \\ + & 59 & + & 4 \\ + & 71 & + & 4 \\ + & 87 & + & 4 \\ + & 82 & + & 4 \\ + & 117 & + & 4 \\ + & 102 & + & 4 \\ + & 119 & + & 4 \\ + & 156 & + & 4 \\ + & 76 & ++ & 4 \\ + & 46 & ++ & 4 \\ + & 53 & ++ & 4 \\ + & 72 & ++ & 4 \\ + & 60 & ++ & 4 \\ + & 78 & ++ & 4 \\ + & 71 & ++ & 4 \\ + & 64 & ++ & 4 \\ + & 82 & ++ & 4 \\ + & 87 & ++ & 4 \\ + & 91 & ++ & 4 \\ + & 56 & ++ & 4 \\ + & 62 & ++ & 4 \\ + & 77 & ++ & 4 \\ + & 92 & ++ & 4 \\ + & 45 & ++ & 4 \\ + & 42 & ++ & 4 \\ + & 51 & ++ & 4 \\ + & 82 & ++ & 4\end{array}$




\begin{tabular}{|c|c|c|c|}
\hline 742 & 47 & $F$ & Breas \\
\hline 743 & 47 & $F$ & Breas \\
\hline 44 & 43 & $F$ & \\
\hline 45 & 43 & $F$ & \\
\hline 16 & 52 & $F$ & \\
\hline & 52 & $F$ & \\
\hline & 42 & $F$ & \\
\hline & 42 & $F$ & \\
\hline & 62 & $F$ & \\
\hline 1 & 62 & $F$ & \\
\hline 2 & 53 & $F$ & \\
\hline 53 & 53 & $F$ & \\
\hline 54 & 45 & $F$ & \\
\hline 55 & 66 & $F$ & \\
\hline 56 & 66 & $F$ & Bre \\
\hline 57 & 50 & $F$ & \\
\hline 58 & 50 & $F$ & $B r \epsilon$ \\
\hline 59 & 48 & $F$ & \\
\hline & 48 & $F$ & $3 r \epsilon$ \\
\hline 61 & 56 & $F$ & \\
\hline 62 & 56 & $F$ & $B r \epsilon$ \\
\hline 63 & 64 & $F$ & \\
\hline 64 & 64 & $F$ & $\mathrm{Bre}$ \\
\hline 65 & 33 & $F$ & \\
\hline 66 & 33 & $F$ & \\
\hline$\$ 7$ & 33 & $F$ & \\
\hline 68 & 33 & $F$ & \\
\hline & 34 & $F$ & \\
\hline 70 & 34 & $F$ & \\
\hline 71 & 22 & $F$ & \\
\hline 772 & 22 & $F$ & \\
\hline 73 & 28 & $F$ & \\
\hline & 28 & $F$ & \\
\hline 775 & 46 & $\mathrm{~F}$ & \\
\hline
\end{tabular}

\begin{tabular}{|c|c|c|c|c|c|c|}
\hline Invasive ductal carcinoma & T2N0MO & 2 & Ila & malignant & +++ & ++ \\
\hline Invasive ductal carcinoma & T2NOMO & 2 & Ila & malignant & +++ & ++ \\
\hline Invasive ductal carcinoma & T2NOMO & 2 & Ila & malignant & +++ & +++ \\
\hline Invasive ductal carcinoma & T2NOMO & 2 & Ila & malignant & + & + \\
\hline Invasive ductal carcinoma & T2NOMO & 2 & Ila & malignant & + & + \\
\hline Invasive ductal carcinoma & T2NOMO & 2 & Ila & malignant & + & - \\
\hline Invasive ductal carcinoma & T2NOMO & 2 & Ila & malignant & + & ++ \\
\hline Invasive ductal carcinoma & T2NOMO & 2 & Ila & malignant & + & ++ \\
\hline Invasive ductal carcinoma & T2NOMO & 2 & Ila & malignant & - & - \\
\hline Invasive ductal carcinoma & T2NOMO & 2 & Ila & malignant & - & - \\
\hline Invasive ductal carcinoma & T2NOMO & 2 & Ila & malignant & + & - \\
\hline Invasive ductal carcinoma & T2NOMO & 2 & Ila & malignant & + & - \\
\hline Invasive ductal carcinoma & T2NOMO & 2 & Ila & malignant & + & - \\
\hline Invasive ductal carcinoma & T2NOMO & 2 & Ila & malignant & ++ & + \\
\hline Invasive ductal carcinoma & T2NOMO & 2 & Ila & malignant & ++ & + \\
\hline Invasive ductal carcinoma & T2NOMO & 2 & Ila & malignant & - & - \\
\hline Invasive ductal carcinoma & T2NOMO & 2 & Ila & malignant & - & - \\
\hline Invasive ductal carcinoma & T2N0MO & 2 & Ila & malignant & +++ & +++ \\
\hline Invasive ductal carcinoma & T2NOMO & 2 & Ila & malignant & +++ & +++ \\
\hline Invasive ductal carcinoma & T2NOMO & 2 & Ila & malignant & ++ & - \\
\hline Invasive ductal carcinoma & T2NOMO & 2 & Ila & malignant & ++ & - \\
\hline Invasive ductal carcinoma & T2NOMO & 2 & Ila & malignant & ++ & ++ \\
\hline Invasive ductal carcinoma & T2NOMO & 2 & Ila & malignant & ++ & ++ \\
\hline Invasive ductal carcinoma & T2NOMO & 2 & Ila & malignant & ++ & ++ \\
\hline Invasive ductal carcinoma & T2NOMO & 2 & Ila & malignant & ++ & ++ \\
\hline Invasive ductal carcinoma & T2NOMO & 2 & Ila & malignant & - & - \\
\hline Invasive ductal carcinoma & T2NOMO & 2 & Ila & malignant & - & - \\
\hline Invasive ductal carcinoma & T2NOMO & 2 & Ila & malignant & +++ & + \\
\hline Invasive ductal carcinoma & T2NOMO & 2 & Ila & malignant & +++ & + \\
\hline Invasive ductal carcinoma & T2NOMO & 2 & Ila & malignant & - & - \\
\hline Invasive ductal carcinoma & T2NOMO & 2 & Ila & malignant & - & - \\
\hline Invasive ductal carcinoma & T2NOMO & 2 & & malignant & ++ & ++ \\
\hline Invasive ductal carcinoma & T2N0MO & 2 & $11 \mathrm{a}$ & malignant & ++ & ++ \\
\hline Invasive ductal carcinoma & T2NOMO & 2 & Ila & malignant & ++ & ++ \\
\hline
\end{tabular}

$\begin{array}{cccc}+ & 73 & ++ & 4 \\ + & 82 & ++ & 4 \\ + & 87 & ++ & 4 \\ + & 65 & ++ & 4 \\ + & 92 & ++ & 4 \\ + & 107 & ++ & 4 \\ + & 63 & ++ & 4 \\ + & 46 & ++ & 4 \\ + & 49 & ++ & 4 \\ + & 51 & ++ & 4 \\ + & 58 & ++ & 4 \\ + & 62 & ++ & 4 \\ + & 71 & ++ & 4 \\ + & 79 & ++ & 4 \\ + & 48 & ++ & 4 \\ + & 53 & ++ & 4 \\ + & 128 & + & 4 \\ + & 112 & + & 4 \\ + & 103 & + & 4 \\ + & 76 & ++ & 4 \\ + & 69 & ++ & 4 \\ + & 61 & ++ & 4 \\ + & 81 & ++ & 4 \\ + & 47 & ++ & 4 \\ + & 43 & ++ & 4 \\ + & 58 & ++ & 4 \\ + & 60 & ++ & 4 \\ + & 102 & ++ & 4 \\ + & 92 & ++ & 4 \\ + & 97 & ++ & 4 \\ + & 88 & ++ & 4 \\ + & 82 & ++ & 4 \\ + & 63 & ++ & 4 \\ + & 59 & ++ & 4 \\ + & & & \end{array}$




$\begin{array}{llll}776 & 46 & \mathrm{~F} & \text { Breast } \\ 777 & 63 & \mathrm{~F} & \text { Breast } \\ 778 & 63 & \mathrm{~F} & \text { Breast } \\ 779 & 69 & \mathrm{~F} & \text { Breast } \\ 780 & 69 & \mathrm{~F} & \text { Breast } \\ 781 & 37 & \mathrm{~F} & \text { Breast } \\ 782 & 37 & \mathrm{~F} & \text { Breast } \\ 783 & 65 & \mathrm{~F} & \text { Breast } \\ 784 & 65 & \mathrm{~F} & \text { Breast } \\ 785 & 50 & \mathrm{~F} & \text { Breast } \\ 786 & 50 & \mathrm{~F} & \text { Breast } \\ 787 & 57 & \mathrm{~F} & \text { Breast } \\ 788 & 57 & \mathrm{~F} & \text { Breast } \\ 789 & 48 & \mathrm{~F} & \text { Breast } \\ 790 & 48 & \mathrm{~F} & \text { Breast } \\ 791 & 62 & \mathrm{~F} & \text { Breast } \\ 792 & 62 & \mathrm{~F} & \text { Breast } \\ 793 & 56 & \mathrm{~F} & \text { Breast } \\ 794 & 56 & \mathrm{~F} & \text { Breast } \\ 795 & 60 & \mathrm{~F} & \text { Breast } \\ 796 & 60 & \mathrm{~F} & \text { Breast } \\ 797 & 60 & \mathrm{~F} & \text { Breast } \\ 798 & 60 & \mathrm{~F} & \text { Breast } \\ 799 & 60 & \mathrm{~F} & \text { Breast } \\ 800 & 60 & \mathrm{~F} & \text { Breast } \\ 801 & 56 & \mathrm{~F} & \text { Breast } \\ 802 & 56 & \mathrm{~F} & \text { Breast } \\ 803 & 51 & \mathrm{~F} & \text { Breast } \\ 804 & 55 & \mathrm{~F} & \text { Breast } \\ 805 & 55 & \mathrm{~F} & \text { Breast } \\ 806 & 45 & \mathrm{~F} & \text { Breast } \\ 807 & 45 & \mathrm{~F} & \text { Breast } \\ 808 & 83 & \mathrm{~F} & \text { Breast } \\ 809 & 83 & \mathrm{~F} & \text { Breast } \\ & & & \end{array}$

\begin{tabular}{|c|c|c|c|c|c|c|}
\hline Invasive ductal carcinoma & T2NOMO & 2 & Ila & malignant & ++ & ++ \\
\hline Invasive ductal carcinoma & T2NOMO & 2 & Ila & malignant & +++ & - \\
\hline Invasive ductal carcinoma & T2N0MO & 2 & Ila & malignant & +++ & - \\
\hline Invasive ductal carcinoma & T2NOMO & 2 & Ila & malignant & + & - \\
\hline Invasive ductal carcinoma & T2NOMO & 2 & Ila & malignant & + & - \\
\hline Invasive ductal carcinoma & T2N0MO & 2 & Ila & malignant & - & - \\
\hline Invasive ductal carcinoma & T2NOMO & 2 & Ila & malignant & - & - \\
\hline Invasive ductal carcinoma & T2NOMO & 2 & Ila & malignant & - & - \\
\hline Invasive ductal carcinoma & T2NOMO & 2 & Ila & malignant & - & - \\
\hline Invasive ductal carcinoma & T2NOMO & 2 & Ila & malignant & - & - \\
\hline Invasive ductal carcinoma & T2NOMO & 2 & Ila & malignant & - & - \\
\hline Invasive ductal carcinoma & T2NOMO & 2 & Ila & malignant & + & - \\
\hline Invasive ductal carcinoma & T2NOMO & 2 & Ila & malignant & + & - \\
\hline Invasive ductal carcinoma & T2NOMO & 2 & Ila & malignant & ++ & + \\
\hline Invasive ductal carcinoma & T2NOMO & 2 & Ila & malignant & ++ & + \\
\hline Invasive ductal carcinoma & T2NOMO & 2 & IIA & Malignant & & \\
\hline Invasive ductal carcinoma & T2NOMO & 2 & IIA & Malignant & & \\
\hline Invasive ductal carcinoma & T2NOMO & 3 & Ila & malignant & - & - \\
\hline Invasive ductal carcinoma & T2NOMO & 3 & Ila & malignant & - & - \\
\hline Invasive ductal carcinoma & T2N0MO & 3 & Ila & malignant & - & - \\
\hline Invasive ductal carcinoma & T2NOMO & 3 & Ila & malignant & - & - \\
\hline Invasive ductal carcinoma & T2N0MO & 3 & Ila & malignant & - & - \\
\hline Invasive ductal carcinoma & T2NOMO & 3 & Ila & malignant & - & - \\
\hline Invasive ductal carcinoma & T2NOMO & 3 & Ila & malignant & - & - \\
\hline Invasive ductal carcinoma & T2N0MO & 3 & Ila & malignant & - & - \\
\hline Invasive ductal carcinoma & T2NOMO & 3 & Ila & malignant & - & - \\
\hline Invasive ductal carcinoma & T2NOMO & 3 & Ila & malignant & - & - \\
\hline Invasive ductal carcinoma & T2NOMO & 3 & Ila & malignant & - & - \\
\hline Invasive ductal carcinoma & T2NOMO & 3 & Ila & malignant & - & - \\
\hline Invasive ductal carcinoma & T2NOMO & 3 & Ila & malignant & - & - \\
\hline Invasive ductal carcinoma & T2NOMO & 3 & Ila & malignant & - & - \\
\hline Invasive ductal carcinoma & T2NOMO & 3 & Ila & malignant & - & - \\
\hline Invasive ductal carcinoma & T2NOMO & 3 & Ila & malignant & - & - \\
\hline Invasive ductal carcinoma & T2NOMO & 3 & Ila & malignant & - & - \\
\hline
\end{tabular}

$\begin{array}{cccc}+ & 87 & ++ & 4 \\ + & 47 & ++ & 4 \\ + & 41 & ++ & 4 \\ + & 52 & ++ & 4 \\ + & 68 & ++ & 4 \\ + & 72 & ++ & 4 \\ + & 63 & ++ & 4 \\ + & 58 & ++ & 4 \\ + & 55 & ++ & 4 \\ + & 62 & ++ & 4 \\ + & 69 & ++ & 4 \\ + & 102 & + & 4 \\ + & 48 & ++ & 4 \\ + & 40 & ++ & 4 \\ + & 52 & ++ & 4 \\ + & 62 & ++ & 4 \\ + & 48 & ++ & 4 \\ + & 51 & ++ & 4 \\ + & 55 & ++ & 4 \\ + & 56 & ++ & 4 \\ + & 73 & ++ & 4 \\ + & 64 & ++ & 4 \\ + & 55 & ++ & 4 \\ + & 71 & ++ & 4 \\ + & 79 & ++ & 4 \\ + & 81 & ++ & 4 \\ + & 76 & ++ & 4 \\ + & 77 & ++ & 4 \\ + & 63 & ++ & 4 \\ + & 65 & ++ & 4 \\ + & 69 & ++ & 4 \\ + & 72 & ++ & 4 \\ + & 75 & + & 4 \\ + & 109 & + & 4 \\ + & & & \end{array}$




$\begin{array}{llll}810 & 43 & \mathrm{~F} & \text { Breast } \\ 811 & 43 & \mathrm{~F} & \text { Breast } \\ 812 & 50 & \mathrm{~F} & \text { Breast } \\ 813 & 50 & \mathrm{~F} & \text { Breast } \\ 814 & 48 & \mathrm{~F} & \text { Breast } \\ 815 & 48 & \mathrm{~F} & \text { Breast } \\ 816 & 64 & \mathrm{~F} & \text { Breast } \\ 817 & 64 & \mathrm{~F} & \text { Breast } \\ 818 & 40 & \mathrm{~F} & \text { Breast } \\ 819 & 40 & \mathrm{~F} & \text { Breast } \\ 820 & 63 & \mathrm{~F} & \text { Breast } \\ 821 & 63 & \mathrm{~F} & \text { Breast } \\ 822 & 58 & \mathrm{~F} & \text { Breast } \\ 823 & 58 & \mathrm{~F} & \text { Breast } \\ 824 & 34 & \mathrm{~F} & \text { Breast } \\ 825 & 34 & \mathrm{~F} & \text { Breast } \\ 826 & 58 & \mathrm{~F} & \text { Breast } \\ 827 & 58 & \mathrm{~F} & \text { Breast } \\ 828 & 49 & \mathrm{~F} & \text { Breast } \\ 829 & 49 & \mathrm{~F} & \text { Breast } \\ 830 & 40 & \mathrm{~F} & \text { Breast } \\ 831 & 40 & \mathrm{~F} & \text { Breast } \\ 832 & 47 & \mathrm{~F} & \text { Breast } \\ 833 & 34 & \mathrm{~F} & \text { Breast } \\ 834 & 34 & \mathrm{~F} & \text { Breast } \\ 835 & 34 & \mathrm{~F} & \text { Breast } \\ 836 & 30 & \mathrm{~F} & \text { Breast } \\ 837 & 30 & \mathrm{~F} & \text { Breast } \\ 838 & 43 & \mathrm{~F} & \text { Breast } \\ 839 & 43 & \mathrm{~F} & \text { Breast } \\ 840 & 36 & \mathrm{~F} & \text { Breast } \\ 841 & 36 & \mathrm{~F} & \text { Breast } \\ 842 & 64 & \mathrm{~F} & \text { Breast } \\ 843 & 64 & \mathrm{~F} & \text { Breast } \\ & & & \end{array}$

\begin{tabular}{|c|c|c|c|c|c|c|}
\hline Invasive ductal carcinoma & T2NOMO & 3 & Ila & malignant & - & - \\
\hline Invasive ductal carcinoma & T2NOMO & 3 & Ila & malignant & - & - \\
\hline Invasive ductal carcinoma & T2NOMO & 3 & Ila & malignant & ++ & ++ \\
\hline Invasive ductal carcinoma & T2NOMO & 3 & Ila & malignant & ++ & ++ \\
\hline Invasive ductal carcinoma & T2N0MO & 3 & Ila & malignant & - & - \\
\hline Invasive ductal carcinoma & T2NOMO & 3 & Ila & malignant & - & - \\
\hline Invasive ductal carcinoma & T2N0MO & 3 & Ila & malignant & - & - \\
\hline Invasive ductal carcinoma & T2NOMO & 3 & Ila & malignant & - & - \\
\hline Invasive ductal carcinoma & T2NOMO & 3 & Ila & malignant & ++ & ++ \\
\hline Invasive ductal carcinoma & T2NOMO & 3 & Ila & malignant & ++ & ++ \\
\hline Invasive ductal carcinoma & T2NOMO & 3 & Ila & malignant & +++ & + \\
\hline Invasive ductal carcinoma & T2NOMO & 3 & Ila & malignant & +++ & + \\
\hline Invasive ductal carcinoma & T2NOMO & 3 & Ila & malignant & - & * \\
\hline Invasive ductal carcinoma & T2NOMO & 3 & Ila & malignant & - & - \\
\hline Invasive ductal carcinoma & T2NOMO & 3 & Ila & malignant & - & * \\
\hline Invasive ductal carcinoma & T2NOMO & 3 & Ila & malignant & - & * \\
\hline Invasive ductal carcinoma & T2NOMO & 3 & Ila & malignant & - & * \\
\hline Invasive ductal carcinoma & T2N0M0 & 3 & Ila & malignant & - & * \\
\hline Invasive ductal carcinoma & T2NOMO & 3 & Ila & malignant & - & - \\
\hline Invasive ductal carcinoma & T2NOMO & 3 & Ila & malignant & - & - \\
\hline Invasive ductal carcinoma & T2NOMO & 3 & Ila & malignant & + & - \\
\hline Invasive ductal carcinoma & T2NOMO & 3 & Ila & malignant & + & - \\
\hline Invasive ductal carcinoma & T2NOMO & 3 & Ila & malignant & - & * \\
\hline Invasive ductal carcinoma & T2N0M0 & 3 & Ila & malignant & - & - \\
\hline Invasive ductal carcinoma & T2NOMO & 3 & Ila & malignant & - & - \\
\hline Invasive ductal carcinoma & T2N0M0 & 3 & Ila & malignant & - & - \\
\hline Invasive ductal carcinoma & T2NOMO & 3 & Ila & malignant & - & - \\
\hline Invasive ductal carcinoma & T2NOMO & 3 & Ila & malignant & - & - \\
\hline Invasive ductal carcinoma & T2NOMO & 3 & Ila & malignant & - & - \\
\hline Invasive ductal carcinoma & T2NOMO & 3 & Ila & malignant & - & - \\
\hline Invasive ductal carcinoma & T2NOMO & 3 & Ila & malignant & - & - \\
\hline Invasive ductal carcinoma & T2NOMO & 3 & Ila & malignant & - & - \\
\hline Invasive ductal carcinoma & T2NOMO & 3 & Ila & malignant & - & - \\
\hline Invasive ductal carcinoma & T2N0MO & 3 & Ila & malignant & - & - \\
\hline
\end{tabular}

$\begin{array}{cccc}+ & 46 & ++ & 4 \\ + & 66 & ++ & 4 \\ + & 71 & ++ & 4 \\ + & 52 & ++ & 4 \\ + & 59 & ++ & 4 \\ + & 73 & ++ & 4 \\ + & 66 & ++ & 4 \\ + & 69 & ++ & 4 \\ + & 119 & + & 4 \\ + & 102 & + & 4 \\ + & 67 & ++ & 4 \\ + & 72 & ++ & 4 \\ + & 48 & ++ & 4 \\ + & 43 & ++ & 4 \\ + & 52 & ++ & 4 \\ + & 59 & ++ & 4 \\ + & 61 & ++ & 4 \\ + & 73 & ++ & 4 \\ + & 84 & ++ & 4 \\ + & 44 & ++ & 4 \\ + & 49 & ++ & 4 \\ + & 63 & ++ & 4 \\ + & 67 & ++ & 4 \\ + & 71 & ++ & 4 \\ + & 88 & + & 4 \\ + & 81 & + & 4 \\ + & 92 & + & 4 \\ + & 46 & ++ & 4 \\ + & 49 & ++ & 4 \\ + & 51 & ++ & 4 \\ + & 58 & ++ & 4 \\ + & 62 & ++ & 4 \\ + & 88 & ++ & 4 \\ + & 74 & ++ & 4 \\ + & & & \end{array}$




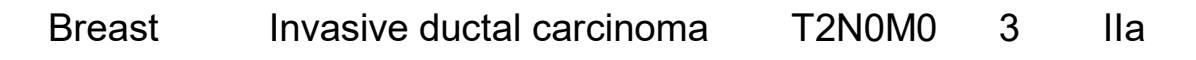

Breast

Breast tal

Breast

ductal carcinoma (breast tis؟ T2NOMO

Bsive ductal carcinoma (breast tis $\leqslant$ T2NOMO

Breast nvasive ductal carcinoma (sparse T2NOMO

Breast nvasive ductal carcinoma (sparse T2NOMO

Breast arcinoma (chronic inflammation of T2NOMO

Breast Ioma (severe atypical hyperplasia T2NOMO

Breast Ioma (severe atypical hyperplasia T2NOM0

Breast nvasive ductal carcinoma (sparse T2NOMO

Breast nvasive ductal carcinoma (sparse T2NOMO

Breast carcinoma (chronic inflammation c T2NOMO

Breast nvasive ductal carcinoma (sparse T2NOMO

Breast tal carcinoma (fibrous tissue and $k$ T2NOMO

Breast sive ductal carcinoma (fibrofatty tis T2NOMO

Breast 2 ductal carcinoma (sparse) with r T2NOMO

Breast sive ductal carcinoma (fibrofatty tis T2NOMO

Breast stal carcinoma (cataplasia and nec T2NOMO

Breast stal carcinoma (cataplasia and nec T2NOMO

Breast nvasive ductal carcinoma (sparse T2NOMO

Breast

Breast

Breast

Breast

Breast

Breast

Breast

Breast

Breast

Breast

Breast

Breast

Breast

$\begin{array}{cccc}+ & 79 & ++ & 4 \\ + & 61 & ++ & 4 \\ + & 54 & ++ & 4 \\ + & 41 & ++ & 4 \\ + & 48 & ++ & 4 \\ + & 43 & ++ & 4 \\ + & 68 & ++ & 4 \\ + & 88 & + & 4 \\ + & 109 & + & 4 \\ + & 92 & + & 4 \\ + & 93 & + & 4 \\ + & 98 & + & 4 \\ + & 118 & + & 4 \\ + & 123 & + & 4 \\ + & 146 & + & 4 \\ + & 156 & + & 4 \\ + & 129 & + & 4 \\ + & 104 & + & 4 \\ + & 108 & + & 4 \\ + & 118 & + & 4 \\ + & 102 & + & 4 \\ + & 87 & ++ & 4 \\ + & 75 & ++ & 4 \\ + & 64 & ++ & 4 \\ + & 48 & ++ & 4 \\ + & 53 & ++ & 4 \\ + & 56 & ++ & 4 \\ + & 71 & ++ & 4 \\ + & 78 & ++ & 4 \\ + & 92 & ++ & 4 \\ + & 67 & ++ & 4 \\ + & 79 & ++ & 4 \\ + & 54 & + & 4 \\ + & 87 & + & 4\end{array}$


Breast Mixed lobular and ductal carcinomi T2N0M0

Breast tpocrine carcinoma (fibrous tissue T2NOMO

Breast

Breast

Breast

Breast

Breast

Breast

Breast

Breast

Breast

Breast

Breast

Breast

Breast

Breast

Breast

Breast

Breast

Breast

Breast

Breast

Breast

Breast

Breast

Breast

Breast

Breast

Breast

Breast

Breast

Breast

Breast

Breast

Apocrine carcinoma T2NOMO

asive ductal carcinoma (breast tis؟ T2NOMO

nvasive ductal carcinoma (sparse T2NOMO

Invasive ductal carcinoma T2NOMO

asive ductal carcinoma (breast tis؟ T2NOMO

ive mixed ductal and lobular carci T2NOMO

ive mixed ductal and lobular carci T2NOMO

ive mixed ductal and lobular carci T2NOMO

ive mixed ductal and lobular carci T2NOMO

\begin{abstract}
Medullary carcinoma
T2NOMO
\end{abstract}

Medullary carcinoma

T2NOMO

Medullary carcinoma

T2NOMO

Carcinoma tissue (sparse)

Medullary carcinoma

T2NOMO

Medullary carcinoma

$\mathrm{T} 2 \mathrm{NOMO}$

T2NOMO

Papillary carcinoma

Papillary carcinoma

Papillary carcinoma

T2NOMO

T2NOMO

T2NOMO

Papillary carcinoma

T2NOMO

asive ductal carcinoma (breast tis؟ T2NOMO

nvasive ductal carcinoma (sparse T2NOMO

nvasive ductal carcinoma (sparse T2NOMO

al carcinoma (blood vessel and ac T2NOMO

al carcinoma (blood vessel and ac T2NOMO

tal carcinoma (fibrous tissue and $k$ T2NOMO

nvasive ductal carcinoma (sparse T2NOMO

nvasive ductal carcinoma (sparse T2NOMO

nvasive ductal carcinoma (sparse T2NOMO

'asive ductal carcinoma (breast dı T2NOM0

ctal carcinoma (inflammatory necr T2NOMO

Medullary carcinoma

T2NOMO

Medullary carcinoma

T2NOMO

$\begin{array}{ccccc}\text { Ila } & \text { malignant } & +++ & - & - \\ \text { Ila } & \text { malignant } & * & * & - \\ \text { Ila } & \text { malignant } & - & * & - \\ \text { Ila } & \text { malignant } & * & * & 0 \\ \text { Ila } & \text { malignant } & - & - & 3+ \\ \text { Ila } & \text { malignant } & * & * & 0 \\ \text { Ila } & \text { malignant } & * & * & 0 \\ \text { Ila } & \text { malignant } & ++ & ++ & 0 \\ \text { Ila } & \text { malignant } & +++ & +++ & 0 \\ \text { Ila } & \text { malignant } & ++ & ++ & 0 \\ \text { Ila } & \text { malignant } & ++ & ++ & 0 \\ \text { Ila } & \text { malignant } & - & - & 0 \\ \text { Ila } & \text { malignant } & - & - & 0 \\ \text { Ila } & \text { malignant } & - & - & 0 \\ \text { Ila } & \text { malignant } & - & - & 0 \\ \text { Ila } & \text { malignant } & - & - & 0 \\ \text { Ila } & \text { malignant } & - & - & 0 \\ \text { Ila } & \text { malignant } & - & - & 3+ \\ \text { Ila } & \text { malignant } & - & - & 3+ \\ \text { Ila } & \text { malignant } & - & - & 0 \\ \text { Ila } & \text { malignant } & - & - & 0 \\ \text { Ila } & \text { malignant } & * & * & 0 \\ \text { Ila } & \text { malignant } & - & - & 0 \\ \text { Ila } & \text { malignant } & ++ & ++ & 0 \\ \text { Ila } & \text { malignant } & * & * & 3+ \\ \text { Ila } & \text { malignant } & * & * & * \\ \text { Ila } & \text { malignant } & * & * & 0 \\ \text { Ila } & \text { malignant } & - & - & 0 \\ \text { Ila } & \text { malignant } & - & - & 0 \\ \text { Ila } & \text { malignant } & +++ & - & 0 \\ \text { Ila } & \text { malignant } & * & * & 0 \\ & - & * & 0 \\ \text { malignant } & ++ & - & 3+ \\ & + & * & 3+ \\ & & & \end{array}$

$\begin{array}{cccc}+ & 89 & + & 4 \\ + & 43 & ++ & 4 \\ + & 52 & ++ & 4 \\ + & 45 & ++ & 4 \\ + & 55 & ++ & 4 \\ + & 52 & ++ & 4 \\ + & 63 & ++ & 4 \\ + & 124 & + & 4 \\ + & 105 & + & 4 \\ + & 99 & + & 4 \\ + & 78 & ++ & 4 \\ + & 71 & ++ & 4 \\ + & 43 & ++ & 4 \\ + & 51 & ++ & 4 \\ + & 57 & ++ & 4 \\ + & 62 & ++ & 4 \\ + & 68 & ++ & 4 \\ + & 54 & ++ & 4 \\ + & 77 & ++ & 4 \\ + & 85 & ++ & 4 \\ + & 44 & ++ & 4 \\ + & 49 & ++ & 4 \\ + & 53 & ++ & 4 \\ + & 87 & ++ & 4 \\ + & 102 & ++ & 4 \\ + & 118 & ++ & 4 \\ + & 67 & ++ & 4 \\ + & 61 & ++ & 4 \\ + & 53 & ++ & 4 \\ + & 49 & ++ & 4 \\ + & 114 & + & 4 \\ + & 129 & + & 4 \\ + & 107 & + & 4 \\ + & 67 & ++ & 4 \\ + & & & \end{array}$


Breast nvasive ductal carcinoma (sparse T2NOMO

Breast asive ductal carcinoma (breast tis $\leqslant$ T2NOMO

Breast al carcinoma (fibrofatty tissue and T2NOMO

Breast al carcinoma (fibrofatty tissue and T2NOMO

Breast nvasive ductal carcinoma (sparse T2NOMO

Breast al carcinoma (fibrofatty tissue and T2NOMO

Breast al carcinoma (fibrofatty tissue and T2NOMO

Breast $\quad$ (chronic inflammation of fibrofatt: T2NOMO

Breast tal carcinoma (fibrous tissue and $k$ T2NOMO

Breast al carcinoma (blood vessel and ac T2NOMO

Breast nvasive ductal carcinoma (sparse T2NOMO

Breast a (chronic inflammation of fibrous T2NOMO

Breast ive ductal carcinoma (tumoral nec T2NOMO

Breast ive ductal carcinoma (tumoral nec T2NOMO

Breast ar carcinoma (fibrofatty tissue and T2NOMO

Breast isive lobular carcinoma (breast tis! T2NOMO

Breast nvasive ductal carcinoma (sparse T2NOMO

Breast al carcinoma (fibrofatty tissue and T2NOMO

Breast ive ductal carcinoma (tumoral nec T2NOMO

Breast tal carcinoma (fibrous tissue and $k$ T2NOMO

Breast iductal carcinoma with early infiltre T2NOMO

Breast iductal carcinoma with early infiltre T2NOMO

Breast a ductal carcinoma (duct of breast T2NOMO

Breast tal carcinoma (fibrous tissue and $k$ T2NOMO

Breast iductal carcinoma with early infiltrc T2NOMO

Breast iductal carcinoma with early infiltrc T2NOMO

Breast tal carcinoma (fibrous tissue and $k$ T2NOMO

Breast nvasive ductal carcinoma (sparse T2NOMO

Breast al carcinoma (fibrofatty tissue and T2NOMO

Breast

Breast

Medullary carcinoma

T2NOMO

Medullary carcinoma

T2NOMO

Atypical medullary carcinoma T2NOMO

Breast

Atypical medullary carcinoma T2NOMO

Breast Mixed lobular and ductal carcinomi T2NOMO

\begin{tabular}{|c|c|c|c|}
\hline Ila & malignant & +++ & +++ \\
\hline Ila & malignant & * & * \\
\hline Ila & malignant & * & * \\
\hline Ila & malignant & * & * \\
\hline Ila & malignant & * & * \\
\hline Ila & malignant & * & * \\
\hline Ila & malignant & * & * \\
\hline Ila & malignant & * & * \\
\hline Ila & malignant & * & * \\
\hline Ila & malignant & * & * \\
\hline Ila & malignant & ++ & + \\
\hline Ila & malignant & - & - \\
\hline Ila & malignant & * & * \\
\hline Ila & malignant & * & * \\
\hline Ila & malignant & * & * \\
\hline Ila & malignant & * & * \\
\hline Ila & malignant & + & - \\
\hline Ila & malignant & * & * \\
\hline Ila & malignant & * & * \\
\hline Ila & malignant & * & * \\
\hline Ila & malignant & + & - \\
\hline Ila & malignant & + & - \\
\hline Ila & malignant & * & * \\
\hline Ila & malignant & * & * \\
\hline Ila & malignant & - & - \\
\hline Ila & malignant & - & - \\
\hline Ila & malignant & * & * \\
\hline Ila & malignant & * & * \\
\hline Ila & malignant & * & * \\
\hline Ila & malignant & - & - \\
\hline Ila & malignant & - & - \\
\hline Ila & malignant & - & - \\
\hline Ila & malignant & - & - \\
\hline Ila & malignant & ++ & - \\
\hline
\end{tabular}

$\begin{array}{cccc}+ & 73 & ++ & 4 \\ + & 105 & + & 4 \\ + & 109 & + & 4 \\ + & 76 & ++ & 4 \\ + & 89 & + & 4 \\ + & 47 & ++ & 4 \\ + & 53 & ++ & 4 \\ + & 116 & + & 4 \\ + & 103 & + & 4 \\ + & 87 & ++ & 4 \\ + & 82 & ++ & 4 \\ + & 45 & ++ & 4 \\ + & 42 & ++ & 4 \\ + & 55 & ++ & 4 \\ + & 58 & ++ & 4 \\ + & 62 & ++ & 4 \\ + & 68 & ++ & 4 \\ + & 42 & ++ & 4 \\ + & 49 & ++ & 4 \\ + & 51 & ++ & 4 \\ + & 48 & ++ & 4 \\ + & 58 & ++ & 4 \\ + & 64 & ++ & 4 \\ + & 59 & ++ & 4 \\ + & 48 & ++ & 4 \\ + & 44 & ++ & 4 \\ + & 52 & ++ & 4 \\ + & 55 & ++ & 4 \\ + & 48 & ++ & 4 \\ + & 63 & ++ & 4 \\ + & 72 & ++ & 4 \\ + & 42 & ++ & 4 \\ + & 76 & ++ & 4 \\ + & 66 & ++ & 4 \\ + & & & \end{array}$

4
4
4
4
4
4
4
4
4
4
4
4
4
4
4
4
4
4
4
4
4
4
4
4
4
4
4
4
4
4
4
4




\begin{tabular}{|c|c|c|c|c|c|c|c|}
\hline Breast & Mixed lobular and ductal carcinomi & T2NOMO & - & Ila & malignant & ++ & - \\
\hline Breast & ısive adenocarcinoma (duct of bre & T2NOMO & - & Ila & malignant & * & * \\
\hline Breast & Invasive adenocarcinoma (sparse' & T2NOMO & - & Ila & malignant & +++ & + \\
\hline Breast & Apocrine carcinoma & T2NOMO & - & Ila & malignant & - & - \\
\hline Breast & Apocrine carcinoma & T2N0MO & - & Ila & malignant & - & - \\
\hline Breast & Carcinoid & T2NOMO & - & Ila & malignant & - & - \\
\hline Breast & Carcinoid & T2N0MO & - & Ila & malignant & - & - \\
\hline Breast & Invasive cribriform carcinoma & T2NOMO & - & Ila & malignant & +++ & +++ \\
\hline Breast & Invasive cribriform carcinoma & T2NOMO & - & Ila & malignant & +++ & +++ \\
\hline Breast & -ipid-rich carcinoma (breast tissue & T2NOMO & - & Ila & malignant & * & * \\
\hline Breast & :arcinoma (fibrofatty tissue and blc & T2NOMO & - & Ila & malignant & * & * \\
\hline Breast & 'cogen-rich carcinoma (breast tiss & T2NOMO & - & Ila & malignant & * & * \\
\hline Breast & ר carcinoma (fibrofatty tissue and & T2NOMO & - & Ila & malignant & * & * \\
\hline Breast & nvasive ductal carcinoma (sparse & T2NOMO & - & Ila & malignant & +++ & * \\
\hline Breast & əsive ductal carcinoma (breast tis & T2NOMO & - & Ila & malignant & * & * \\
\hline Breast & Medullary carcinoma & T2NOMO & - & Ila & malignant & - & - \\
\hline Breast & Medullary carcinoma & T2NOMO & - & Ila & malignant & - & - \\
\hline Breast & Medullary carcinoma & T2NOMO & - & Ila & malignant & - & - \\
\hline Breast & Medullary carcinoma & T2NOMO & - & Ila & malignant & - & - \\
\hline Breast & Medullary carcinoma & T2NOMO & - & Ila & malignant & ++ & ++ \\
\hline Breast & Medullary carcinoma & T2NOMO & - & Ila & malignant & ++ & ++ \\
\hline Breast & Invasive lobular carcinoma & T2NOMO & - & Ila & malignant & +++ & - \\
\hline Breast & Invasive lobular carcinoma & T2NOMO & - & Ila & malignant & +++ & + \\
\hline Breast & Mixed lobular and duct carcinoma & T2NOMO & - & Ila & malignant & + & + \\
\hline Breast & Mixed lobular and duct carcinoma & T2NOMO & - & Ila & malignant & + & + \\
\hline Breast & Invasive papillary carcinoma & T2NOMO & - & Ila & malignant & ++ & + \\
\hline Breast & Invasive papillary carcinoma & T2N0M0 & - & Ila & malignant & ++ & + \\
\hline Breast & Invasive papillary carcinoma & T2NOMO & - & Ila & malignant & +++ & + \\
\hline Breast & Invasive papillary carcinoma & T2N0M0 & - & Ila & malignant & +++ & + \\
\hline Breast & ve lobular carcinoma (cataplasia t & T2N0MO & & IIA & malignant & & \\
\hline Breast & Invasive lobular carcinoma & T2N0M0 & & IIA & malignant & & \\
\hline Breast & Invasive lobular carcinoma & T2NOMO & & IIA & malignant & & \\
\hline Breast & ve lobular carcinoma (cataplasia t & T2NOMO & & IIA & malignant & & \\
\hline Breast & Invasive lobular carcinoma & T2NOMO & & IIA & malignant & & \\
\hline
\end{tabular}

$\begin{array}{cccc}+ & 63 & ++ & 4 \\ + & 78 & ++ & 4 \\ + & 53 & ++ & 4 \\ + & 58 & ++ & 4 \\ + & 60 & ++ & 4 \\ + & 70 & ++ & 4 \\ + & 62 & ++ & 4 \\ + & 67 & ++ & 4 \\ + & 69 & ++ & 4 \\ + & 54 & ++ & 4 \\ + & 59 & ++ & 4 \\ + & 103 & + & 4 \\ + & 89 & + & 4 \\ + & 115 & + & 4 \\ + & 98 & + & 4 \\ + & 47 & ++ & 4 \\ + & 49 & ++ & 4 \\ + & 51 & ++ & 4 \\ + & 57 & ++ & 4 \\ + & 63 & ++ & 4 \\ + & 72 & ++ & 4 \\ + & 70 & ++ & 4 \\ + & 83 & ++ & 4 \\ + & 87 & ++ & 4 \\ + & 93 & + & 4 \\ + & 96 & + & 4 \\ + & 54 & ++ & 4 \\ + & 48 & ++ & 4 \\ + & 67 & ++ & 4 \\ + & 63 & ++ & 4 \\ + & 75 & ++ & 4 \\ + & 82 & ++ & 4 \\ + & 89 & ++ & 4 \\ + & 65 & ++ & 4\end{array}$




\begin{tabular}{|c|c|c|c|c|c|c|c|c|c|c|c|c|c|c|}
\hline 980 & 47 & $\mathrm{~F}$ & Breast & Invasive lobular carcinoma & T2N0MO & & IIA & malignant & & & & + & 81 & ++ \\
\hline 981 & 69 & $\mathrm{~F}$ & Breast & Invasive lobular carcinoma & T2NOMO & & IIA & malignant & & & & + & 41 & ++ \\
\hline 982 & 58 & $\mathrm{~F}$ & Breast & Invasive lobular carcinoma & T2NOMO & & IIA & malignant & & & & + & 44 & ++ \\
\hline 983 & 60 & $\mathrm{~F}$ & Breast & Invasive lobular carcinoma & T2NOMO & & IIA & malignant & & & & + & 50 & ++ \\
\hline 984 & 57 & $\mathrm{~F}$ & Breast & Invasive lobular carcinoma & T2NOMO & & IIA & malignant & & & & + & 52 & ++ \\
\hline 985 & 53 & $\mathrm{~F}$ & breast & re ductal carcinoma, mucinous ca & $\mathrm{T} 2 \mathrm{~N} 1 \mathrm{M} 0$ & 3 & $\mathrm{Ilb}$ & Malignant & & & & + & 63 & ++ \\
\hline 986 & 50 & $\mathrm{~F}$ & breast & infiltrative ductal carcinoma & $\mathrm{T} 2 \mathrm{~N} 1 \mathrm{M} 0$ & 3 & Illb & Malignant & & & & + & 143 & ++ \\
\hline 987 & 45 & $\mathrm{~F}$ & breast & infiltrative ductal carcinoma & T2N1M0 & 3 & Ilb & Malignant & & & & + & 125 & ++ \\
\hline 988 & 43 & $\mathrm{~F}$ & breast & infiltrative ductal carcinoma & T2N1M0 & $2--3$ & IIb & Malignant & & & & + & 116 & ++ \\
\hline 989 & 54 & $\mathrm{~F}$ & breast & infiltrative ductal carcinoma & T2N1M0 & 2 & Ilb & Malignant & & & & + & 72 & ++ \\
\hline 990 & 40 & $\mathrm{~F}$ & breast & infiltrative ductal carcinoma & $\mathrm{T} 2 \mathrm{~N} 1 \mathrm{M} 0$ & 2 & Ilb & Malignant & & & & + & 89 & +++ \\
\hline 991 & 41 & $\mathrm{~F}$ & breast & infiltrative ductal carcinoma & $\mathrm{T} 2 \mathrm{~N} 1 \mathrm{M} 0$ & 2 & Ilb & Malignant & & & & + & 54 & +++ \\
\hline 992 & 41 & $\mathrm{~F}$ & breast & infiltrative ductal carcinoma & $\mathrm{T} 2 \mathrm{~N} 1 \mathrm{M} 0$ & 2 & Ilb & Malignant & & & & + & 58 & ++ \\
\hline 993 & 46 & $\mathrm{~F}$ & breast & infiltrative ductal carcinoma & $\mathrm{T} 2 \mathrm{~N} 1 \mathrm{M} 0$ & 2 & Ilb & Malignant & & & & + & 47 & ++ \\
\hline 994 & 46 & $\mathrm{~F}$ & breast & infiltrative ductal carcinoma & $\mathrm{T} 2 \mathrm{~N} 1 \mathrm{M} 0$ & 2 & Ilb & Malignant & & & & + & 112 & + \\
\hline 995 & 52 & $\mathrm{~F}$ & breast & infiltrative ductal carcinoma & $\mathrm{T} 2 \mathrm{~N} 1 \mathrm{M} 0$ & 2 & Ilb & Malignant & & & & + & 107 & + \\
\hline 996 & 51 & $\mathrm{~F}$ & breast & infiltrative ductal carcinoma & $\mathrm{T} 2 \mathrm{~N} 1 \mathrm{M} 0$ & 2 & Ilb & Malignant & & & & + & 113 & + \\
\hline 997 & 49 & $\mathrm{~F}$ & breast & infiltrative ductal carcinoma & $\mathrm{T} 2 \mathrm{~N} 1 \mathrm{M} 0$ & 3 & Ilb & Malignant & & & & + & 67 & +++ \\
\hline 998 & 44 & $\mathrm{~F}$ & breast & infiltrative ductal carcinoma & $\mathrm{T} 2 \mathrm{~N} 1 \mathrm{M} 0$ & 3 & Ilb & Malignant & & & & + & 43 & +++ \\
\hline 999 & 44 & $\mathrm{~F}$ & breast & infiltrating lobular carcinoma & $\mathrm{T} 2 \mathrm{~N} 1 \mathrm{M} 0$ & 3 & IIb & Malignant & & & & + & 49 & +++ \\
\hline 1000 & 49 & $\mathrm{~F}$ & breast & infiltrating lobular carcinoma & $\mathrm{T} 2 \mathrm{~N} 1 \mathrm{M} 0$ & 3 & IIb & Malignant & & & & + & 52 & +++ \\
\hline 1001 & 46 & $\mathrm{~F}$ & Breast & Invasive ductal carcinoma & T3NOMO & 1 & Ilb & malignant & - & +++ & 0 & + & 78 & ++ \\
\hline 1002 & 46 & $\mathrm{~F}$ & Breast & Invasive ductal carcinoma & T3NOMO & 1 & IIb & malignant & - & +++ & 0 & + & 84 & ++ \\
\hline 1003 & 56 & $\mathrm{~F}$ & Breast & Invasive ductal carcinoma & T3NOMO & 1 & $\mathrm{IIb}$ & malignant & ++ & + & 0 & + & 57 & ++ \\
\hline 1004 & 40 & $\mathrm{~F}$ & Breast & Invasive ductal carcinoma & T3NOMO & 1 & IIb & malignant & + & * & 0 & + & 51 & ++ \\
\hline 1005 & 36 & $\mathrm{~F}$ & Breast & Invasive ductal carcinoma & T3NOMO & 1 & $\mathrm{IIb}$ & malignant & +++ & * & 0 & + & 60 & ++ \\
\hline 1006 & 37 & $\mathrm{~F}$ & Breast & Invasive ductal carcinoma & T2NOMO & 1 & $\mathrm{Ilb}$ & malignant & - & - & $2+$ & + & 72 & ++ \\
\hline 1007 & 37 & $\mathrm{~F}$ & Breast & Invasive ductal carcinoma & T2NOMO & 1 & IIb & malignant & - & - & $2+$ & + & 81 & ++ \\
\hline 1008 & 58 & $\mathrm{~F}$ & Breast & Invasive ductal carcinoma & T2NOMO & 1 & IIb & malignant & - & - & $2+$ & + & 46 & ++ \\
\hline 1009 & 52 & $\mathrm{~F}$ & Breast & Invasive ductal carcinoma & T3NOMO & 1 & $\mathrm{Ilb}$ & malignant & - & * & $2+$ & + & 49 & ++ \\
\hline 1010 & 52 & $\mathrm{~F}$ & Breast & Invasive ductal carcinoma & T3NOMO & 1 & IIb & malignant & - & * & $2+$ & + & 55 & ++ \\
\hline 1011 & 44 & $\mathrm{~F}$ & Breast & Invasive ductal carcinoma & T3N1M0 & 1 & Ilb & malignant & + & - & 0 & + & 43 & ++ \\
\hline 1012 & 44 & $\mathrm{~F}$ & Breast & Invasive ductal carcinoma & T3N1M0 & 1 & Ilb & malignant & + & - & 0 & + & 48 & ++ \\
\hline 1013 & 49 & $\mathrm{~F}$ & Breast & Invasive ductal carcinoma & T3NOMO & 2 & Ilb & malignant & +++ & + & - & + & 87 & ++ \\
\hline
\end{tabular}




\begin{tabular}{|c|c|c|c|c|c|c|c|c|c|c|c|c|c|c|}
\hline 1014 & 49 & $\mathrm{~F}$ & Breast & Invasive ductal carcinoma & T3N0M0 & 2 & Ilb & malignant & +++ & + & - & + & 82 & ++ \\
\hline 1015 & 48 & $\mathrm{~F}$ & Breast & Invasive ductal carcinoma & $\mathrm{T} 2 \mathrm{~N} 1 \mathrm{M} 0$ & 2 & IIb & malignant & + & + & - & + & 91 & ++ \\
\hline 1016 & 48 & $\mathrm{~F}$ & Breast & Invasive ductal carcinoma & $\mathrm{T} 2 \mathrm{~N} 1 \mathrm{M} 0$ & 2 & IIb & malignant & + & + & - & + & 67 & ++ \\
\hline 1017 & 40 & $\mathrm{~F}$ & Breast & Invasive ductal carcinoma & $\mathrm{T} 2 \mathrm{~N} 1 \mathrm{M} 0$ & 2 & IIb & malignant & +++ & + & - & + & 73 & ++ \\
\hline 1018 & 40 & $\mathrm{~F}$ & Breast & Invasive ductal carcinoma & $\mathrm{T} 2 \mathrm{~N} 1 \mathrm{M} 0$ & 2 & IIb & malignant & +++ & + & - & + & 56 & ++ \\
\hline 1019 & 78 & $\mathrm{~F}$ & Breast & Invasive ductal carcinoma & $\mathrm{T} 2 \mathrm{~N} 1 \mathrm{M} 0$ & 2 & $\mathrm{IIb}$ & malignant & ++ & - & - & + & 48 & ++ \\
\hline 1020 & 78 & $\mathrm{~F}$ & Breast & Invasive ductal carcinoma & $\mathrm{T} 2 \mathrm{~N} 1 \mathrm{M} 0$ & 2 & $\mathrm{IIb}$ & malignant & ++ & - & - & + & 52 & ++ \\
\hline 1021 & 46 & $\mathrm{~F}$ & Breast & Invasive ductal carcinoma & T3NOMO & 2 & IIb & malignant & ++ & - & - & + & 54 & ++ \\
\hline 1022 & 46 & $\mathrm{~F}$ & Breast & Invasive ductal carcinoma & T3NOMO & 2 & IIb & malignant & ++ & - & - & + & 68 & ++ \\
\hline 1023 & 50 & $\mathrm{~F}$ & Breast & Invasive ductal carcinoma & $\mathrm{T} 2 \mathrm{~N} 1 \mathrm{M} 0$ & 2 & IIb & malignant & - & - & - & + & 49 & ++ \\
\hline 1024 & 53 & $\mathrm{~F}$ & Breast & Invasive ductal carcinoma & $\mathrm{T} 2 \mathrm{~N} 1 \mathrm{M} 0$ & 2 & IIb & malignant & - & - & $1+$ & + & 51 & ++ \\
\hline 1025 & 53 & $\mathrm{~F}$ & Breast & Invasive ductal carcinoma & $\mathrm{T} 2 \mathrm{~N} 1 \mathrm{M} 0$ & 2 & IIb & malignant & - & - & $2+$ & + & 62 & ++ \\
\hline 1026 & 65 & $\mathrm{~F}$ & Breast & Invasive ductal carcinoma & $\mathrm{T} 2 \mathrm{~N} 1 \mathrm{M} 0$ & 2 & Ilb & malignant & - & - & $3+$ & + & 67 & ++ \\
\hline 1027 & 65 & $\mathrm{~F}$ & Breast & Invasive ductal carcinoma & $\mathrm{T} 2 \mathrm{~N} 1 \mathrm{M} 0$ & 2 & Ilb & malignant & - & - & $3+$ & + & 72 & ++ \\
\hline 1028 & 80 & $\mathrm{~F}$ & Breast & Invasive ductal carcinoma & T3NOMO & 2 & IIb & malignant & +++ & - & - & + & 84 & ++ \\
\hline 1029 & 80 & $\mathrm{~F}$ & Breast & Invasive ductal carcinoma & T3NOMO & 2 & $\mathrm{IIb}$ & malignant & +++ & * & - & + & 103 & ++ \\
\hline 1030 & 49 & $\mathrm{~F}$ & Breast & Invasive ductal carcinoma & $\mathrm{T} 2 \mathrm{~N} 1 \mathrm{M} 0$ & 2 & Ilb & malignant & * & ++ & 0 & + & 118 & ++ \\
\hline 1031 & 49 & $\mathrm{~F}$ & Breast & Invasive ductal carcinoma & $\mathrm{T} 2 \mathrm{~N} 1 \mathrm{M} 0$ & 2 & IIb & malignant & * & ++ & 0 & + & 47 & ++ \\
\hline 1032 & 45 & $\mathrm{~F}$ & Breast & Invasive ductal carcinoma & $\mathrm{T} 2 \mathrm{~N} 1 \mathrm{M} 0$ & 2 & IIb & malignant & - & - & 0 & + & 87 & ++ \\
\hline 1033 & 45 & $\mathrm{~F}$ & Breast & Invasive ductal carcinoma & $\mathrm{T} 2 \mathrm{~N} 1 \mathrm{M} 0$ & 2 & IIb & malignant & - & - & 0 & + & 92 & ++ \\
\hline 1034 & 62 & $\mathrm{~F}$ & Breast & Invasive ductal carcinoma & $\mathrm{T} 2 \mathrm{~N} 1 \mathrm{M} 0$ & 2 & IIb & malignant & ++ & ++ & 0 & + & 46 & ++ \\
\hline 1035 & 62 & $\mathrm{~F}$ & Breast & Invasive ductal carcinoma & $\mathrm{T} 2 \mathrm{~N} 1 \mathrm{M} 0$ & 2 & Ilb & malignant & ++ & ++ & 0 & + & 55 & ++ \\
\hline 1036 & 34 & $\mathrm{~F}$ & Breast & Invasive ductal carcinoma & T3NOMO & 2 & IIb & malignant & - & - & $2+$ & + & 43 & ++ \\
\hline 1037 & 34 & $\mathrm{~F}$ & Breast & Invasive ductal carcinoma & T3NOMO & 2 & $\mathrm{IIb}$ & malignant & - & - & $2+$ & + & 48 & ++ \\
\hline 1038 & 48 & $\mathrm{~F}$ & Breast & Invasive ductal carcinoma & T3NOMO & 2 & IIb & malignant & +++ & +++ & 0 & + & 96 & ++ \\
\hline 1039 & 48 & $\mathrm{~F}$ & Breast & Invasive ductal carcinoma & T3NOMO & 2 & $\mathrm{IIb}$ & malignant & +++ & +++ & 0 & + & 108 & ++ \\
\hline 1040 & 38 & $\mathrm{~F}$ & Breast & Invasive ductal carcinoma & $\mathrm{T} 2 \mathrm{~N} 1 \mathrm{M} 0$ & 2 & $\mathrm{Ilb}$ & malignant & + & - & 0 & + & 87 & ++ \\
\hline 1041 & 38 & $\mathrm{~F}$ & Breast & Invasive ductal carcinoma & $\mathrm{T} 2 \mathrm{~N} 1 \mathrm{M} 0$ & 2 & IIb & malignant & + & - & 0 & + & 73 & ++ \\
\hline 1042 & 48 & $\mathrm{~F}$ & Breast & nvasive ductal carcinoma (sparse & $\mathrm{T} 2 \mathrm{~N} 1 \mathrm{M} 0$ & 2 & IIb & malignant & - & - & 0 & + & 49 & ++ \\
\hline 1043 & 48 & $\mathrm{~F}$ & Breast & Invasive ductal carcinoma & $\mathrm{T} 2 \mathrm{~N} 1 \mathrm{M} 0$ & 2 & $\mathrm{Ilb}$ & malignant & - & - & 0 & + & 54 & ++ \\
\hline 1044 & 61 & $\mathrm{~F}$ & Breast & asive ductal carcinoma with necrc & $\mathrm{T} 2 \mathrm{~N} 1 \mathrm{M} 0$ & 2 & IIb & malignant & - & - & 0 & + & 57 & ++ \\
\hline 1045 & 61 & $\mathrm{~F}$ & Breast & asive ductal carcinoma with necrc & $\mathrm{T} 2 \mathrm{~N} 1 \mathrm{M} 0$ & 2 & Ilb & malignant & - & - & * & + & 64 & ++ \\
\hline 1046 & 50 & $\mathrm{~F}$ & Breast & Invasive ductal carcinoma & $\mathrm{T} 2 \mathrm{~N} 1 \mathrm{M} 0$ & 2 & Ilb & malignant & - & - & 0 & + & 42 & ++ \\
\hline 1047 & 50 & $\mathrm{~F}$ & Breast & nvasive ductal carcinoma (sparse & $\mathrm{T} 2 \mathrm{~N} 1 \mathrm{M} 0$ & 2 & IIb & malignant & - & - & 0 & + & 48 & ++ \\
\hline
\end{tabular}




\begin{tabular}{|c|c|c|c|c|c|c|c|c|c|c|}
\hline 1048 & 73 & $\mathrm{~F}$ & Breast & Invasive ductal carcinoma & $\mathrm{T} 2 \mathrm{~N} 1 \mathrm{M} 0$ & 2 & Ilb & malignant & - & - \\
\hline 1049 & 73 & $\mathrm{~F}$ & Breast & Invasive ductal carcinoma & $\mathrm{T} 2 \mathrm{~N} 1 \mathrm{M} 0$ & 2 & IIb & malignant & - & - \\
\hline 050 & 56 & $\mathrm{~F}$ & Breast & Invasive ductal carcinoma & T2N1M0 & 2 & Ilb & malignant & - & - \\
\hline 1051 & 56 & $\mathrm{~F}$ & Breast & Invasive ductal carcinoma & T2N1M0 & 2 & Ilb & malignant & - & - \\
\hline 1052 & 46 & $\mathrm{~F}$ & Breast & Invasive ductal carcinoma & T3NOMO & 2 & Ilb & malignant & - & - \\
\hline 1053 & 46 & $\mathrm{~F}$ & Breast & Invasive ductal carcinoma & T3NOMO & 2 & IIb & malignant & - & - \\
\hline 1054 & 39 & $\mathrm{~F}$ & Breast & Invasive ductal carcinoma & $\mathrm{T} 2 \mathrm{~N} 1 \mathrm{M} 0$ & 2 & $\mathrm{Ilb}$ & malignant & + & + \\
\hline 1055 & 39 & $\mathrm{~F}$ & Breast & Invasive ductal carcinoma & $\mathrm{T} 2 \mathrm{~N} 1 \mathrm{M} 0$ & 2 & IIb & malignant & + & + \\
\hline 1056 & 32 & $\mathrm{~F}$ & Breast & Invasive ductal carcinoma & T3N0MO & 2 & IIb & malignant & - & - \\
\hline 1057 & 32 & $\mathrm{~F}$ & Breast & Invasive ductal carcinoma & T3NOMO & 2 & IIb & malignant & - & - \\
\hline 1058 & 47 & $\mathrm{~F}$ & Breast & Invasive ductal carcinoma & T3NOMO & 2 & IIb & malignant & ++ & ++ \\
\hline 1059 & 47 & $\mathrm{~F}$ & Breast & Invasive ductal carcinoma & T3NOMO & 2 & IIb & malignant & ++ & ++ \\
\hline 1060 & 48 & $\mathrm{~F}$ & Breast & Invasive ductal carcinoma & T3NOMO & 2 & IIb & malignant & - & - \\
\hline 1061 & 48 & $\mathrm{~F}$ & Breast & Invasive ductal carcinoma & T3NOMO & 2 & IIb & malignant & - & - \\
\hline 1062 & 35 & $\mathrm{~F}$ & Breast & Invasive ductal carcinoma & T3NOMO & 2 & IIb & malignant & ++ & +++ \\
\hline 1063 & 49 & $\mathrm{~F}$ & Breast & Invasive ductal carcinoma & T2NOMO & 2 & IIb & malignant & - & - \\
\hline 1064 & 49 & $\mathrm{~F}$ & Breast & Invasive ductal carcinoma & T2NOMO & 2 & IIb & malignant & - & - \\
\hline 1065 & 60 & $\mathrm{~F}$ & Breast & Invasive ductal carcinoma & T3NOMO & 2 & IIb & malignant & - & - \\
\hline 1066 & 60 & $\mathrm{~F}$ & Breast & Invasive ductal carcinoma & T3NOMO & 2 & IIb & malignant & - & - \\
\hline 1067 & 35 & $\mathrm{~F}$ & Breast & Invasive ductal carcinoma & T2NOMO & 2 & IIb & malignant & ++ & - \\
\hline 1068 & 35 & $\mathrm{~F}$ & Breast & Invasive ductal carcinoma & T2NOMO & 2 & IIb & malignant & ++ & - \\
\hline 1069 & 43 & $\mathrm{~F}$ & Breast & Invasive ductal carcinoma & $\mathrm{T} 2 \mathrm{~N} 1 \mathrm{M} 0$ & 2 & Ilb & malignant & ++ & - \\
\hline 1070 & 43 & $\mathrm{~F}$ & Breast & Invasive ductal carcinoma & $\mathrm{T} 2 \mathrm{~N} 1 \mathrm{M} 0$ & 2 & Ilb & malignant & ++ & - \\
\hline 1071 & 41 & $\mathrm{~F}$ & Breast & Invasive ductal carcinoma & $\mathrm{T} 2 \mathrm{~N} 1 \mathrm{M} 0$ & 2 & Ilb & malignant & +++ & - \\
\hline 1072 & 41 & $\mathrm{~F}$ & Breast & Invasive ductal carcinoma & $\mathrm{T} 2 \mathrm{~N} 1 \mathrm{M} 0$ & 2 & Ilb & malignant & +++ & - \\
\hline 1073 & 38 & $\mathrm{~F}$ & Breast & Invasive ductal carcinoma & T3N1M0 & 2 & IIb & malignant & - & - \\
\hline 1074 & 38 & $\mathrm{~F}$ & Breast & Invasive ductal carcinoma & T3N1M0 & 2 & Ilb & malignant & - & - \\
\hline 1075 & 36 & $\mathrm{~F}$ & Breast & Invasive ductal carcinoma & T3NOMO & 2 & Ilb & malignant & ++ & ++ \\
\hline 1076 & 36 & $\mathrm{~F}$ & Breast & Invasive ductal carcinoma & T3NOMO & 2 & Ilb & malignant & ++ & ++ \\
\hline 1077 & 28 & $\mathrm{~F}$ & Breast & Invasive ductal carcinoma & T3NOMO & 2 & IIb & malignant & ++ & ++ \\
\hline 1078 & 61 & $\mathrm{~F}$ & Breast & Invasive ductal carcinoma & $\mathrm{T} 2 \mathrm{~N} 1 \mathrm{M} 0$ & 2 & Ilb & malignant & + & - \\
\hline 1079 & 61 & $\mathrm{~F}$ & Breast & Invasive ductal carcinoma & $\mathrm{T} 2 \mathrm{~N} 1 \mathrm{M} 0$ & 2 & Ilb & malignant & - & - \\
\hline 1080 & 44 & $\mathrm{~F}$ & Breast & asive ductal carcinoma with necrc & $\mathrm{T} 2 \mathrm{~N} 1 \mathrm{M} 0$ & 2 & Ilb & malignant & ++ & ++ \\
\hline 1081 & 44 & $\mathrm{~F}$ & Breast & asive ductal carcinoma with necrc & $\mathrm{T} 2 \mathrm{~N} 1 \mathrm{M} 0$ & 2 & $\mathrm{IIb}$ & malignant & ++ & ++ \\
\hline
\end{tabular}




\begin{tabular}{|c|c|c|c|c|c|c|c|c|c|c|c|c|c|c|}
\hline 1082 & 54 & $\mathrm{~F}$ & Breast & Invasive ductal carcinoma & T3N0M0 & 2 & IIb & malignant & +++ & + & 0 & + & 68 & ++ \\
\hline 1083 & 54 & $\mathrm{~F}$ & Breast & Invasive ductal carcinoma & T3NOMO & 2 & IIb & malignant & +++ & + & 0 & + & 73 & ++ \\
\hline 1084 & 41 & $\mathrm{~F}$ & Breast & nvasive ductal carcinoma (sparse & T3NOMO & 2 & Ilb & malignant & - & - & * & + & 77 & ++ \\
\hline 1085 & 41 & $\mathrm{~F}$ & Breast & nvasive ductal carcinoma (sparse & T3NOMO & 2 & IIb & malignant & - & - & 0 & + & 63 & ++ \\
\hline 1086 & 47 & $\mathrm{~F}$ & Breast & Invasive ductal carcinoma & T3NOMO & 2 & IIb & malignant & +++ & +++ & 0 & + & 66 & ++ \\
\hline 1087 & 47 & $\mathrm{~F}$ & Breast & Invasive ductal carcinoma & T3NOMO & 2 & $\mathrm{IIb}$ & malignant & +++ & +++ & 0 & + & 58 & ++ \\
\hline 1088 & 41 & $\mathrm{~F}$ & Breast & Invasive ductal carcinoma & T3NOMO & 2 & $\mathrm{IIb}$ & malignant & ++ & ++ & $2+$ & + & 62 & ++ \\
\hline 1089 & 41 & $\mathrm{~F}$ & Breast & Invasive ductal carcinoma & T3NOMO & 2 & IIb & malignant & ++ & ++ & $2+$ & + & 49 & ++ \\
\hline 1090 & 54 & $\mathrm{~F}$ & Breast & ə ductal carcinoma (sparse) with $r$ & T3NOMO & 2 & IIb & malignant & +++ & - & 0 & + & 47 & ++ \\
\hline 1091 & 54 & $\mathrm{~F}$ & Breast & Invasive ductal carcinoma & T3NOMO & 2 & IIb & malignant & +++ & + & 0 & + & 58 & ++ \\
\hline 1092 & 51 & $\mathrm{~F}$ & Breast & Invasive ductal carcinoma & T2N1M0 & 2 & IIb & malignant & + & - & 0 & + & 64 & ++ \\
\hline 1093 & 51 & $\mathrm{~F}$ & Breast & Invasive ductal carcinoma & $\mathrm{T} 2 \mathrm{~N} 1 \mathrm{M} 0$ & 2 & IIb & malignant & + & - & 0 & + & 69 & ++ \\
\hline 1094 & 54 & $\mathrm{~F}$ & Breast & Invasive ductal carcinoma & T3N0MO & 2 & IIb & malignant & + & - & $3+$ & + & 42 & ++ \\
\hline 1095 & 54 & $\mathrm{~F}$ & Breast & Invasive ductal carcinoma & T3NOMO & 2 & Ilb & malignant & + & - & $3+$ & + & 48 & ++ \\
\hline 1096 & 53 & $\mathrm{~F}$ & Breast & Invasive ductal carcinoma & T3NOMO & 2 & IIb & malignant & - & - & $2+$ & + & 51 & ++ \\
\hline 1097 & 53 & $\mathrm{~F}$ & Breast & asive ductal carcinoma with necrc & T3NOMO & 2 & Ilb & malignant & - & - & $2+$ & + & 54 & ++ \\
\hline 1098 & 52 & $\mathrm{~F}$ & Breast & Invasive ductal carcinoma & T3NOMO & 2 & Ilb & malignant & - & - & 0 & + & 48 & ++ \\
\hline 1099 & 52 & $\mathrm{~F}$ & Breast & Invasive ductal carcinoma & T3NOMO & 2 & Ilb & malignant & - & - & 0 & + & 55 & ++ \\
\hline 1100 & 44 & $\mathrm{~F}$ & Breast & Invasive ductal carcinoma & $\mathrm{T} 2 \mathrm{~N} 1 \mathrm{M} 0$ & 2 & IIb & malignant & - & - & 0 & + & 69 & ++ \\
\hline 1101 & 44 & $\mathrm{~F}$ & Breast & Invasive ductal carcinoma & $\mathrm{T} 2 \mathrm{~N} 1 \mathrm{M} 0$ & 2 & IIb & malignant & - & - & 0 & + & 89 & ++ \\
\hline 1102 & 54 & $\mathrm{~F}$ & Breast & Invasive ductal carcinoma & $\mathrm{T} 2 \mathrm{~N} 1 \mathrm{M} 0$ & 2 & IIb & malignant & - & - & $2+$ & + & 92 & ++ \\
\hline 1103 & 54 & $\mathrm{~F}$ & Breast & Invasive ductal carcinoma & $\mathrm{T} 2 \mathrm{~N} 1 \mathrm{M} 0$ & 2 & Ilb & malignant & - & - & $2+$ & + & 96 & ++ \\
\hline 1104 & 58 & $\mathrm{~F}$ & Breast & Invasive ductal carcinoma & T3NOMO & 2 & IIb & malignant & - & - & $2+$ & + & 42 & ++ \\
\hline 1105 & 58 & $\mathrm{~F}$ & Breast & Invasive ductal carcinoma & T3NOMO & 2 & Ilb & malignant & - & - & $2+$ & + & 44 & ++ \\
\hline 1106 & 46 & $\mathrm{~F}$ & Breast & Invasive ductal carcinoma & $\mathrm{T} 2 \mathrm{~N} 1 \mathrm{M} 0$ & 2 & IIb & malignant & - & + & $2+$ & + & 53 & ++ \\
\hline 1107 & 46 & $\mathrm{~F}$ & Breast & Invasive ductal carcinoma & $\mathrm{T} 2 \mathrm{~N} 1 \mathrm{M} 0$ & 2 & IIb & malignant & - & + & $2+$ & + & 87 & ++ \\
\hline 1108 & 81 & $\mathrm{~F}$ & Breast & Invasive ductal carcinoma & $\mathrm{T} 2 \mathrm{~N} 1 \mathrm{M} 0$ & 2 & $\mathrm{Ilb}$ & malignant & ++ & - & 0 & + & 94 & ++ \\
\hline 1109 & 81 & $\mathrm{~F}$ & Breast & Invasive ductal carcinoma & $\mathrm{T} 2 \mathrm{~N} 1 \mathrm{M} 0$ & 2 & IIb & malignant & ++ & + & 0 & + & 66 & ++ \\
\hline 1110 & 60 & $\mathrm{~F}$ & Breast & Invasive ductal carcinoma & T3NOMO & 2 & IIb & malignant & + & + & $1+$ & + & 57 & ++ \\
\hline 1111 & 60 & $\mathrm{~F}$ & Breast & Invasive ductal carcinoma & T3NOMO & 2 & $\mathrm{Ilb}$ & malignant & + & + & $1+$ & + & 85 & ++ \\
\hline 1112 & 43 & $\mathrm{~F}$ & Breast & Invasive ductal carcinoma & T3NOMO & 2 & IIb & malignant & - & - & 0 & + & 47 & ++ \\
\hline 1113 & 44 & $\mathrm{~F}$ & Breast & Invasive ductal carcinoma & $\mathrm{T} 2 \mathrm{~N} 1 \mathrm{M} 0$ & 2 & Ilb & malignant & - & - & $3+$ & + & 88 & ++ \\
\hline 1114 & 46 & $\mathrm{~F}$ & Breast & Invasive ductal carcinoma & T3NOMO & 2 & Ilb & malignant & - & - & 0 & + & 46 & ++ \\
\hline 1115 & 60 & $\mathrm{~F}$ & Breast & Invasive ductal carcinoma & T3NOMO & 2 & IIb & malignant & +++ & + & 0 & + & 98 & ++ \\
\hline
\end{tabular}




\begin{tabular}{|c|c|c|c|c|c|c|c|c|c|c|c|c|c|c|}
\hline 1116 & 60 & $\mathrm{~F}$ & Breast & Invasive ductal carcinoma & T3N0MO & 2 & $\mathrm{Ilb}$ & malignant & +++ & + & 0 & + & 105 & ++ \\
\hline 1117 & 63 & $\mathrm{~F}$ & Breast & Invasive ductal carcinoma & T3NOMO & 2 & IIb & malignant & - & - & 0 & + & 98 & ++ \\
\hline 1118 & 63 & $\mathrm{~F}$ & Breast & Invasive ductal carcinoma & T3NOMO & 2 & IIb & malignant & - & - & 0 & + & 116 & ++ \\
\hline 1119 & 57 & $\mathrm{~F}$ & Breast & Invasive ductal carcinoma & T3N1M0 & 2 & $\mathrm{IIb}$ & malignant & + & - & 0 & + & 78 & ++ \\
\hline 1120 & 57 & $\mathrm{~F}$ & Breast & Invasive ductal carcinoma & T3N1M0 & 2 & $\mathrm{Ilb}$ & malignant & + & - & 0 & + & 83 & ++ \\
\hline 1121 & 60 & $\mathrm{~F}$ & Breast & Invasive ductal carcinoma & T3NOMO & 2 & $\mathrm{Ilb}$ & malignant & - & - & 0 & + & 79 & ++ \\
\hline 1122 & 60 & $\mathrm{~F}$ & Breast & Invasive ductal carcinoma & T3NOMO & 2 & Ilb & malignant & - & - & 0 & + & 47 & ++ \\
\hline 1123 & 46 & $\mathrm{~F}$ & Breast & Invasive ductal carcinoma & T3NOMO & 2 & Ilb & malignant & - & - & 0 & + & 52 & ++ \\
\hline 1124 & 46 & $\mathrm{~F}$ & Breast & Invasive ductal carcinoma & T3NOMO & 2 & Ilb & malignant & - & - & 0 & + & 56 & ++ \\
\hline 1125 & 41 & $\mathrm{~F}$ & Breast & Invasive ductal carcinoma & T3N1M0 & 2 & IIb & malignant & ++ & + & 0 & + & 64 & ++ \\
\hline 1126 & 41 & $\mathrm{~F}$ & Breast & Invasive ductal carcinoma & T3N1M0 & 2 & Ilb & malignant & ++ & + & 0 & + & 78 & ++ \\
\hline 1127 & 48 & $\mathrm{~F}$ & Breast & Invasive ductal carcinoma & T3NOMO & 2 & Ilb & malignant & + & +++ & 0 & + & 66 & ++ \\
\hline 1128 & 66 & $\mathrm{~F}$ & Breast & Invasive ductal carcinoma & T3NOMO & 2 & IIB & Malignant & & & & + & 41 & ++ \\
\hline 1129 & 48 & $\mathrm{~F}$ & Breast & Invasive ductal carcinoma & T3NOMO & 2 & IIB & Malignant & & & & + & 46 & ++ \\
\hline 1130 & 66 & $\mathrm{~F}$ & Breast & Invasive ductal carcinoma & T3NOMO & 2 & IIB & Malignant & & & & + & 76 & ++ \\
\hline 1131 & 48 & $\mathrm{~F}$ & Breast & Invasive ductal carcinoma & T3NOMO & 2 & IIB & Malignant & & & & + & 73 & ++ \\
\hline 1132 & 45 & $\mathrm{~F}$ & Breast & Invasive ductal carcinoma & $\mathrm{T} 2 \mathrm{~N} 1 \mathrm{M} 0$ & 3 & Ilb & malignant & - & - & $3+$ & + & 56 & ++ \\
\hline 1133 & 45 & $\mathrm{~F}$ & Breast & Invasive ductal carcinoma & $\mathrm{T} 2 \mathrm{~N} 1 \mathrm{M} 0$ & 3 & Ilb & malignant & - & - & $3+$ & + & 45 & ++ \\
\hline 1134 & 39 & $\mathrm{~F}$ & Breast & Invasive ductal carcinoma & T3NOMO & 3 & IIb & malignant & + & + & $3+$ & + & 85 & ++ \\
\hline 1135 & 39 & $\mathrm{~F}$ & Breast & Invasive ductal carcinoma & T3NOMO & 3 & IIb & malignant & + & + & $3+$ & + & 93 & ++ \\
\hline 1136 & 33 & $\mathrm{~F}$ & Breast & Invasive ductal carcinoma & $\mathrm{T} 2 \mathrm{~N} 1 \mathrm{M} 0$ & 3 & IIb & malignant & - & - & 0 & + & 102 & ++ \\
\hline 1137 & 33 & $\mathrm{~F}$ & Breast & Invasive ductal carcinoma & $\mathrm{T} 2 \mathrm{~N} 1 \mathrm{M} 0$ & 3 & $\mathrm{IIb}$ & malignant & - & - & 0 & + & 99 & ++ \\
\hline 1138 & 45 & $\mathrm{~F}$ & Breast & Invasive ductal carcinoma & T3NOMO & 3 & IIb & malignant & - & - & 0 & + & 117 & ++ \\
\hline 1139 & 45 & $\mathrm{~F}$ & Breast & Invasive ductal carcinoma & T3NOMO & 3 & $\mathrm{Ilb}$ & malignant & - & * & 0 & + & 106 & ++ \\
\hline 1140 & 34 & $\mathrm{~F}$ & Breast & Invasive ductal carcinoma & T3NOMO & 3 & $\mathrm{Ilb}$ & malignant & - & * & 0 & + & 102 & ++ \\
\hline 1141 & 34 & $\mathrm{~F}$ & Breast & Invasive ductal carcinoma & T3NOMO & 3 & $\mathrm{Ilb}$ & malignant & * & * & 0 & + & 94 & ++ \\
\hline 1142 & 34 & $\mathrm{~F}$ & Breast & Invasive ductal carcinoma & T3N0MO & 3 & $\mathrm{Ilb}$ & malignant & - & - & 0 & + & 116 & ++ \\
\hline 1143 & 34 & $\mathrm{~F}$ & Breast & Invasive ductal carcinoma & T3N0MO & 3 & IIb & malignant & - & - & 0 & + & 108 & ++ \\
\hline 1144 & 45 & $\mathrm{~F}$ & Breast & Invasive ductal carcinoma & $\mathrm{T} 2 \mathrm{~N} 1 \mathrm{M} 0$ & 3 & Ilb & malignant & ++ & + & $2+$ & + & 127 & ++ \\
\hline 1145 & 45 & $\mathrm{~F}$ & Breast & Invasive ductal carcinoma & $\mathrm{T} 2 \mathrm{~N} 1 \mathrm{M} 0$ & 3 & $\mathrm{Ilb}$ & malignant & ++ & + & $2+$ & + & 118 & ++ \\
\hline 1146 & 52 & $\mathrm{~F}$ & Breast & əsive ductal carcinoma (breast tis؟ & $\mathrm{T} 2 \mathrm{~N} 1 \mathrm{M} 0$ & - & Ilb & malignant & * & * & - & + & 103 & ++ \\
\hline 1147 & 52 & $\mathrm{~F}$ & Breast & əsive ductal carcinoma (breast tis؟ & $\mathrm{T} 2 \mathrm{~N} 1 \mathrm{M} 0$ & - & Ilb & malignant & * & * & $2+$ & + & 98 & ++ \\
\hline 1148 & 50 & $\mathrm{~F}$ & Breast & nvasive ductal carcinoma (sparse & $\mathrm{T} 2 \mathrm{~N} 1 \mathrm{M} 0$ & - & IIb & malignant & - & - & - & + & 46 & ++ \\
\hline 1149 & 51 & $\mathrm{~F}$ & Breast & nvasive ductal carcinoma (sparse & $\mathrm{T} 2 \mathrm{~N} 1 \mathrm{M} 0$ & - & $\mathrm{Ilb}$ & malignant & + & + & 0 & + & 48 & ++ \\
\hline
\end{tabular}




\begin{tabular}{|c|c|c|c|c|c|c|c|c|c|c|c|c|c|c|}
\hline 1150 & 51 & $\mathrm{~F}$ & Breast & nvasive ductal carcinoma (sparse & $\mathrm{T} 2 \mathrm{~N} 1 \mathrm{M} 0$ & - & Ilb & malignant & + & + & 0 & + & 51 & ++ \\
\hline 1151 & 48 & $\mathrm{~F}$ & Breast & Invasive lobular carcinoma & $\mathrm{T} 2 \mathrm{~N} 1 \mathrm{M} 0$ & - & Illb & malignant & - & - & 0 & + & 63 & ++ \\
\hline 1152 & 48 & $\mathrm{~F}$ & Breast & Invasive lobular carcinoma & $\mathrm{T} 2 \mathrm{~N} 1 \mathrm{M} 0$ & - & $\mathrm{Ilb}$ & malignant & - & - & 0 & + & 77 & ++ \\
\hline 1153 & 35 & $\mathrm{~F}$ & Breast & nvasive ductal carcinoma (sparse & T3NOMO & - & $\mathrm{Ilb}$ & malignant & ++ & +++ & $2+$ & + & 98 & ++ \\
\hline 1154 & 39 & $\mathrm{~F}$ & Breast & Medullary carcinoma & $\mathrm{T} 2 \mathrm{~N} 1 \mathrm{M} 0$ & - & Ilb & malignant & - & - & 0 & + & 48 & ++ \\
\hline 1155 & 39 & $\mathrm{~F}$ & Breast & Medullary carcinoma & $\mathrm{T} 2 \mathrm{~N} 1 \mathrm{M} 0$ & - & Ilb & malignant & - & - & 0 & + & 57 & ++ \\
\hline 1156 & 49 & $\mathrm{~F}$ & Breast & Medullary carcinoma & T3NOMO & - & Ilb & malignant & - & * & 0 & + & 55 & ++ \\
\hline 1157 & 49 & $\mathrm{~F}$ & Breast & Medullary carcinoma & T3NOMO & - & Ilb & malignant & - & * & 0 & + & 43 & ++ \\
\hline 1158 & 28 & $\mathrm{~F}$ & Breast & ㅋl carcinoma (fibrofatty tissue and & T3NOMO & - & IIb & malignant & * & * & * & + & 62 & ++ \\
\hline 1159 & 44 & $\mathrm{~F}$ & Breast & Invasive lobular carcinoma & T3NOMO & - & IIb & malignant & + & + & 0 & + & 67 & ++ \\
\hline 1160 & 44 & $\mathrm{~F}$ & Breast & Invasive lobular carcinoma & T3NOMO & - & Ilb & malignant & + & + & 0 & + & 68 & ++ \\
\hline 1161 & 56 & $\mathrm{~F}$ & Breast & æsive ductal carcinoma (breast tis؟ & T3NOMO & - & $\mathrm{Ilb}$ & malignant & * & * & 0 & + & 72 & ++ \\
\hline 1162 & 43 & $\mathrm{~F}$ & Breast & al carcinoma (fibrofatty tissue and & T3NOMO & - & Ilb & malignant & * & * & * & + & 83 & ++ \\
\hline 1163 & 44 & $\mathrm{~F}$ & Breast & al carcinoma (fibrofatty tissue and & $\mathrm{T} 2 \mathrm{~N} 1 \mathrm{M} 0$ & - & Ilb & malignant & * & * & * & + & 109 & ++ \\
\hline 1164 & 46 & $\mathrm{~F}$ & Breast & ə ductal carcinoma (sparse) with $r$ & T3NOMO & - & Ilb & malignant & - & - & 0 & + & 112 & ++ \\
\hline 1165 & 55 & $\mathrm{~F}$ & Breast & Medullary carcinoma & T3NOMO & - & Ilb & malignant & - & - & 0 & + & 94 & ++ \\
\hline 1166 & 55 & $\mathrm{~F}$ & Breast & Medullary carcinoma & T3NOMO & - & Ilb & malignant & - & - & 0 & + & 47 & ++ \\
\hline 1167 & 52 & $\mathrm{~F}$ & Breast & Invasive lobular carcinoma & $\mathrm{T} 2 \mathrm{~N} 1 \mathrm{M} 0$ & - & IIb & malignant & - & - & 0 & + & 63 & ++ \\
\hline 1168 & 52 & $\mathrm{~F}$ & Breast & Invasive lobular carcinoma & $\mathrm{T} 2 \mathrm{~N} 1 \mathrm{M} 0$ & - & $\mathrm{IIb}$ & malignant & - & - & 0 & + & 52 & ++ \\
\hline 1169 & 40 & $\mathrm{~F}$ & Breast & tal carcinoma (fibrous tissue and $k$ & T3NOMO & - & IIb & malignant & * & * & 0 & + & 43 & ++ \\
\hline 1170 & 58 & $\mathrm{~F}$ & Breast & al carcinoma (adipose tissue and & T2NOMO & - & $\mathrm{IIb}$ & malignant & * & * & * & + & 68 & ++ \\
\hline 1171 & 48 & $\mathrm{~F}$ & Breast & Invasive ductal carcinoma & T3N0MO & - & $\mathrm{Ilb}$ & malignant & * & * & 0 & + & 84 & ++ \\
\hline 1172 & 52 & $\mathrm{~F}$ & Breast & Mixed lobular and duct carcinoma & $\mathrm{T} 2 \mathrm{~N} 1 \mathrm{M} 0$ & - & $\mathrm{IIb}$ & malignant & +++ & + & 0 & + & 47 & ++ \\
\hline 1173 & 52 & $\mathrm{~F}$ & Breast & Mixed lobular and duct carcinoma & $\mathrm{T} 2 \mathrm{~N} 1 \mathrm{M} 0$ & - & Ilb & malignant & +++ & + & 0 & + & 43 & ++ \\
\hline 1174 & 37 & $\mathrm{~F}$ & Breast & Sarcoma phyllodes & T2NOMO & - & IIb & malignant & - & - & 0 & + & 63 & ++ \\
\hline 1175 & 37 & $\mathrm{~F}$ & Breast & Sarcoma phyllodes & T2NOMO & - & Ilb & malignant & - & - & 0 & + & 67 & ++ \\
\hline 1176 & 31 & $F$ & Breast & Sarcoma phyllodes & T2NOMO & - & Ilb & malignant & - & - & 0 & + & 69 & ++ \\
\hline 1177 & 31 & $\mathrm{~F}$ & Breast & Sarcoma phyllodes & T2NOMO & - & IIb & malignant & - & - & 0 & + & 72 & ++ \\
\hline 1178 & 40 & $\mathrm{~F}$ & Breast & Sarcoma phyllodes & T2NOMO & - & Ilb & malignant & - & * & 0 & + & 73 & ++ \\
\hline 1179 & 40 & $\mathrm{~F}$ & Breast & Sarcoma phyllodes & T2NOMO & - & Ilb & malignant & - & * & 0 & + & 84 & ++ \\
\hline 1180 & 50 & $\mathrm{~F}$ & Breast & Mucinous carcinoma & $\mathrm{T} 2 \mathrm{~N} 1 \mathrm{M} 0$ & $1--2$ & $\mathrm{Ilb}$ & malignant & - & - & - & + & 89 & ++ \\
\hline 1181 & 50 & $\mathrm{~F}$ & Breast & Mucinous carcinoma & $\mathrm{T} 2 \mathrm{~N} 1 \mathrm{M} 0$ & $1--2$ & $\mathrm{Ilb}$ & malignant & + & + & - & + & 63 & ++ \\
\hline 1182 & 36 & $\mathrm{~F}$ & Breast & Invasive ductal carcinoma & T3NOMO & $1--2$ & Ilb & malignant & +++ & * & 0 & + & 71 & ++ \\
\hline 1183 & 58 & $\mathrm{~F}$ & Breast & Invasive ductal carcinoma & T3NOMO & $1--2$ & IIB & Malignant & & & & + & 66 & ++ \\
\hline
\end{tabular}




\begin{tabular}{|c|c|c|c|c|c|c|c|c|c|c|c|c|c|c|}
\hline 1184 & 58 & $F$ & Breast & Invasive ductal carcinoma & T3N0M0 & $1--2$ & IIB & Malignant & & & & + & 42 & ++ \\
\hline 1185 & 47 & $\mathrm{~F}$ & Breast & Invasive lobular carcinoma & $\mathrm{T} 2 \mathrm{~N} 1 \mathrm{M} 0$ & & IIB & malignant & & & & + & 73 & ++ \\
\hline 1186 & 35 & $\mathrm{~F}$ & Breast & Invasive lobular carcinoma & T3NOMO & & IIB & malignant & & & & + & 68 & ++ \\
\hline 1187 & 19 & $\mathrm{~F}$ & Breast & Invasive lobular carcinoma & $\mathrm{T} 2 \mathrm{~N} 1 \mathrm{M} 0$ & & IIB & malignant & & & & + & 75 & ++ \\
\hline 1188 & 45 & $\mathrm{~F}$ & Breast & Invasive lobular carcinoma & $\mathrm{T} 2 \mathrm{~N} 1 \mathrm{M} 0$ & & IIB & malignant & & & & + & 58 & ++ \\
\hline 1189 & 49 & $\mathrm{~F}$ & Breast & Invasive lobular carcinoma & $\mathrm{T} 2 \mathrm{~N} 1 \mathrm{M} 0$ & & IIB & malignant & & & & + & 56 & ++ \\
\hline 1190 & 63 & $\mathrm{~F}$ & Breast & Invasive lobular carcinoma & T3NOMO & & IIB & malignant & & & & + & 64 & ++ \\
\hline 1191 & 42 & $\mathrm{~F}$ & Breast & Invasive lobular carcinoma & $\mathrm{T} 2 \mathrm{~N} 1 \mathrm{M} 0$ & & IIB & malignant & & & & + & 68 & ++ \\
\hline 1192 & 50 & $\mathrm{~F}$ & Breast & Invasive lobular carcinoma & $\mathrm{T} 2 \mathrm{~N} 1 \mathrm{M} 0$ & & IIB & malignant & & & & + & 56 & ++ \\
\hline 1193 & 25 & $\mathrm{~F}$ & Breast & Invasive ductal carcinoma & T3NOMO & 2 & IIB & Malignant & +++ & - & $3+$ & + & 45 & ++ \\
\hline 1194 & 39 & $\mathrm{~F}$ & Breast & Invasive ductal carcinoma & $\mathrm{T} 2 \mathrm{~N} 1 \mathrm{M} 0$ & 1 & IIB & Malignant & +++ & ++ & $3+$ & + & 43 & ++ \\
\hline 1195 & 25 & $\mathrm{~F}$ & Breast & Invasive ductal carcinoma & T3NOMO & 2 & IIB & Malignant & +++ & - & $3+$ & + & 56 & ++ \\
\hline 1196 & 39 & $\mathrm{~F}$ & Breast & Invasive ductal carcinoma & $\mathrm{T} 2 \mathrm{~N} 1 \mathrm{M} 0$ & - & IIB & Malignant & * & * & * & + & 73 & ++ \\
\hline 1197 & 35 & $\mathrm{~F}$ & Breast & Invasive ductal carcinoma & T3N2M0 & 2 & IIla & malignant & ++ & ++ & 0 & + & 182 & ++ \\
\hline 1198 & 35 & $\mathrm{~F}$ & Breast & Invasive ductal carcinoma & T3N2M0 & 2 & IIla & malignant & ++ & ++ & 0 & + & 167 & ++ \\
\hline 1199 & 73 & $\mathrm{~F}$ & Breast & Invasive ductal carcinoma & T3N1M0 & 2 & IIla & malignant & - & - & 0 & + & 96 & ++ \\
\hline 1200 & 73 & $\mathrm{~F}$ & Breast & Invasive ductal carcinoma & T3N1M0 & 2 & IIla & malignant & - & - & 0 & + & 103 & ++ \\
\hline 1201 & 41 & $\mathrm{~F}$ & Breast & Invasive ductal carcinoma & $\mathrm{T} 2 \mathrm{~N} 2 \mathrm{M} 0$ & 2 & IIla & malignant & - & - & 0 & + & 119 & ++ \\
\hline 1202 & 41 & $\mathrm{~F}$ & Breast & Invasive ductal carcinoma & $\mathrm{T} 2 \mathrm{~N} 2 \mathrm{M} 0$ & 2 & IIla & malignant & - & - & 0 & + & 87 & ++ \\
\hline 1203 & 57 & $\mathrm{~F}$ & Breast & Invasive ductal carcinoma & T3N1M0 & 2 & IIla & malignant & ++ & - & 0 & + & 93 & ++ \\
\hline 1204 & 57 & $\mathrm{~F}$ & Breast & Invasive ductal carcinoma & T3N1M0 & 2 & Illa & malignant & ++ & - & 0 & + & 104 & ++ \\
\hline 1205 & 63 & $\mathrm{~F}$ & Breast & asive ductal carcinoma with necrc & T3N1M0 & 2 & Illa & malignant & - & - & 0 & + & 117 & ++ \\
\hline 1206 & 63 & $\mathrm{~F}$ & Breast & asive ductal carcinoma with necrc & T3N1M0 & 2 & IIla & malignant & - & - & 0 & + & 101 & ++ \\
\hline 1207 & 44 & $\mathrm{~F}$ & Breast & Invasive ductal carcinoma & $\mathrm{T} 2 \mathrm{~N} 2 \mathrm{M} 0$ & 2 & Illa & malignant & - & - & 0 & + & 113 & ++ \\
\hline 1208 & 44 & $\mathrm{~F}$ & Breast & Invasive ductal carcinoma & $\mathrm{T} 2 \mathrm{~N} 2 \mathrm{M} 0$ & 2 & Illa & malignant & - & - & 0 & + & 87 & ++ \\
\hline 1209 & 33 & $\mathrm{~F}$ & Breast & Invasive ductal carcinoma & T4NOMO & 2 & Illa & malignant & ++ & - & 0 & + & 96 & ++ \\
\hline 1210 & 33 & $\mathrm{~F}$ & Breast & Invasive ductal carcinoma & T4NOMO & 2 & Illa & malignant & ++ & - & 0 & + & 90 & ++ \\
\hline 1211 & 40 & $\mathrm{~F}$ & Breast & Invasive ductal carcinoma & T3N2M0 & 2 & Illa & malignant & - & - & 0 & + & 103 & ++ \\
\hline 1212 & 40 & $\mathrm{~F}$ & Breast & Invasive ductal carcinoma & T3N2M0 & 2 & Illa & malignant & - & - & 0 & + & 108 & ++ \\
\hline 1213 & 44 & $\mathrm{~F}$ & Breast & Invasive ductal carcinoma & T3N2M0 & 3 & Illa & malignant & - & - & 0 & + & 112 & ++ \\
\hline 1214 & 44 & $\mathrm{~F}$ & Breast & Invasive ductal carcinoma & T3N2M0 & 3 & Illa & malignant & - & - & 0 & + & 117 & ++ \\
\hline 1215 & 49 & $\mathrm{~F}$ & Breast & Invasive lobular carcinoma & T3N2M0 & - & Illa & malignant & - & - & 0 & + & 137 & ++ \\
\hline 1216 & 49 & $\mathrm{~F}$ & Breast & Invasive lobular carcinoma & T3N2M0 & - & IIIa & malignant & - & - & 0 & + & 125 & ++ \\
\hline 1217 & 50 & $\mathrm{~F}$ & Breast & Invasive lobular carcinoma & $\mathrm{T} 2 \mathrm{~N} 2 \mathrm{M} 0$ & & IIIA & malignant & & & & + & 128 & ++ \\
\hline
\end{tabular}




$\begin{array}{llll}1218 & 84 & \mathrm{~F} & \text { Breast } \\ 1219 & 84 & \mathrm{~F} & \text { Breast } \\ 1220 & 52 & \mathrm{~F} & \text { Breast } \\ 1221 & 52 & \mathrm{~F} & \text { Breast } \\ 1222 & 48 & \mathrm{~F} & \text { Breast } \\ 1223 & 48 & \mathrm{~F} & \text { Breast } \\ 1224 & 69 & \mathrm{~F} & \text { Breast } \\ 1225 & 69 & \mathrm{~F} & \text { Breast } \\ 1226 & 45 & \mathrm{~F} & \text { Breast } \\ 1227 & 45 & \mathrm{~F} & \text { Breast } \\ 1228 & 32 & \mathrm{~F} & \text { Breast } \\ 1229 & 32 & \mathrm{~F} & \text { Breast } \\ 1230 & 66 & \mathrm{~F} & \text { Breast } \\ 1231 & 66 & \mathrm{~F} & \text { Breast } \\ 1232 & 60 & \mathrm{~F} & \text { Breast } \\ 1233 & 60 & \mathrm{~F} & \text { Breast } \\ 1234 & 64 & \mathrm{~F} & \text { Breast } \\ 1235 & 64 & \mathrm{~F} & \text { Breast } \\ 1236 & 52 & \mathrm{~F} & \text { Breast } \\ 1237 & 52 & \mathrm{~F} & \text { Breast } \\ 1238 & 46 & \mathrm{~F} & \text { Breast } \\ 1239 & 46 & \mathrm{~F} & \text { Breast } \\ 1240 & 65 & \mathrm{~F} & \text { Breast } \\ 1241 & 65 & \mathrm{~F} & \text { Breast } \\ 1242 & 71 & \mathrm{~F} & \text { Breast } \\ 1243 & 71 & \mathrm{~F} & \text { Breast } \\ 1244 & 45 & \mathrm{~F} & \text { Breast } \\ 1245 & 45 & \mathrm{~F} & \text { Breast } \\ 1246 & 40 & \mathrm{~F} & \text { Breast } \\ 1247 & 40 & \mathrm{~F} & \text { Breast } \\ 1248 & 82 & \mathrm{~F} & \text { Breast } \\ 1249 & 82 & \mathrm{~F} & \text { Breast } \\ 1250 & 59 & \mathrm{~F} & \text { Breast } \\ 1251 & 59 & \mathrm{~F} & \text { Breast } \\ & & & \end{array}$

Invasive ductal carcinoma

Invasive ductal carcinoma

Invasive ductal carcinoma

Invasive ductal carcinoma

Invasive ductal carcinoma

Invasive ductal carcinoma

Invasive ductal carcinoma

Invasive ductal carcinoma

Invasive ductal carcinoma

Invasive ductal carcinoma

Invasive ductal carcinoma

Invasive ductal carcinoma

Invasive ductal carcinoma

Invasive ductal carcinoma

Invasive ductal carcinoma

Invasive ductal carcinoma

Invasive ductal carcinoma

Invasive ductal carcinoma

Invasive ductal carcinoma

Invasive ductal carcinoma

Invasive ductal carcinoma

Invasive ductal carcinoma

Invasive ductal carcinoma

Invasive ductal carcinoma

Invasive ductal carcinoma

Invasive ductal carcinoma

Invasive ductal carcinoma

Invasive ductal carcinoma

Invasive ductal carcinoma

Invasive ductal carcinoma

Invasive ductal carcinoma

Invasive ductal carcinoma

Invasive ductal carcinoma

Invasive ductal carcinoma

\begin{tabular}{|c|c|c|c|c|c|}
\hline T4N0M0 & 1 & IIlb & malignant & * & ++ \\
\hline T4NOMO & 1 & IIIb & malignant & $*$ & ++ \\
\hline T4NOMO & 2 & IIIb & malignant & ++ & ++ \\
\hline T4NOMO & 2 & IIIb & malignant & ++ & ++ \\
\hline T4NOMO & 2 & IIIb & malignant & +++ & +++ \\
\hline T4NOMO & 2 & IIIb & malignant & +++ & +++ \\
\hline T4N0M0 & 2 & IIIb & malignant & +++ & - \\
\hline T4N0M0 & 2 & IIIb & malignant & +++ & + \\
\hline T4NOMO & 2 & IIIb & malignant & - & - \\
\hline T4NOMO & 2 & IIIb & malignant & - & - \\
\hline T4NOMO & 2 & IIIb & malignant & + & ++ \\
\hline T4NOMO & 2 & IIlb & malignant & + & ++ \\
\hline T4NOMO & 2 & IIIb & malignant & - & - \\
\hline T4N0M0 & 2 & IIlb & malignant & - & - \\
\hline T4N1M0 & 2 & IIIb & malignant & + & - \\
\hline T4N1M0 & 2 & IIIb & malignant & + & - \\
\hline T4N1M0 & 2 & IIIb & malignant & +++ & +++ \\
\hline T4N1M0 & 2 & IIIb & malignant & +++ & +++ \\
\hline T4NOMO & 2 & IIIb & malignant & - & ++ \\
\hline T4NOMO & 2 & IIIb & malignant & - & ++ \\
\hline T4NOMO & 2 & IIIb & malignant & ++ & - \\
\hline T4NOMO & 2 & IIIb & malignant & ++ & - \\
\hline T4NOMO & 2 & IIIb & malignant & * & ++ \\
\hline T4NOMO & 2 & IIIb & malignant & * & ++ \\
\hline T4N1M0 & 2 & IIIb & malignant & - & - \\
\hline T4N1M0 & 2 & IIIb & malignant & - & - \\
\hline T4N1M0 & 2 & IIIb & malignant & ++ & - \\
\hline T4N1M0 & 2 & IIIb & malignant & ++ & - \\
\hline T4NOMO & 2 & IIIb & malignant & ++ & ++ \\
\hline T4NOMO & 2 & IIIb & malignant & ++ & ++ \\
\hline T4NOM0 & 2 & IIIb & malignant & +++ & - \\
\hline T4NOMO & 2 & IIIb & malignant & +++ & - \\
\hline T4N1M0 & 2 & IIIb & malignant & +++ & - \\
\hline T4N1M0 & 2 & Illb & malignant & +++ & - \\
\hline
\end{tabular}

$\begin{array}{cccc}+ & 120 & ++ & 4 \\ + & 113 & ++ & 4 \\ + & 132 & ++ & 4 \\ + & 143 & ++ & 4 \\ + & 145 & ++ & 4 \\ + & 187 & ++ & 4 \\ + & 165 & ++ & 4 \\ + & 152 & + & 4 \\ + & 132 & + & 4 \\ + & 128 & + & 4 \\ + & 133 & + & 4 \\ + & 144 & + & 4 \\ + & 162 & + & 4 \\ + & 102 & + & 4 \\ + & 97 & + & 4 \\ + & 104 & + & 4 \\ + & 118 & + & 4 \\ + & 103 & + & 4 \\ + & 67 & ++ & 4 \\ + & 58 & ++ & 4 \\ + & 102 & ++ & 4 \\ + & 98 & +++ & 4 \\ + & 93 & +++ & 4 \\ + & 86 & +++ & 4 \\ + & 77 & +++ & 4 \\ + & 57 & +++ & 4 \\ + & 49 & +++ & 4 \\ + & 84 & +++ & 4 \\ + & 76 & +++ & 4 \\ + & 79 & +++ & 4 \\ + & 58 & +++ & 4 \\ + & 53 & +++ & 4 \\ + & 103 & ++ & 4 \\ + & 124 & ++ & 4\end{array}$




\begin{tabular}{|c|c|c|c|c|c|c|c|c|c|c|c|c|c|c|}
\hline 1252 & 63 & $\mathrm{~F}$ & Breast & Invasive ductal carcinoma & T4N0MO & 2 & Illb & malignant & * & - & 0 & + & 73 & +++ \\
\hline 1253 & 63 & $\mathrm{~F}$ & Breast & Invasive ductal carcinoma & T4NOMO & 2 & IIIb & malignant & * & - & 0 & + & 81 & +++ \\
\hline 1254 & 40 & $\mathrm{~F}$ & Breast & asive ductal carcinoma with necrc & T4NOMO & 2 & IIIb & malignant & ++ & ++ & 0 & + & 99 & +++ \\
\hline 1255 & 40 & $\mathrm{~F}$ & Breast & asive ductal carcinoma with necrc & T4NOMO & 2 & IIIb & malignant & ++ & ++ & 0 & + & 94 & +++ \\
\hline 1256 & 81 & $\mathrm{~F}$ & Breast & Invasive ductal carcinoma & T4N0M0 & 2 & IIIb & malignant & +++ & - & 0 & + & 103 & ++ \\
\hline 1257 & 81 & $\mathrm{~F}$ & Breast & Invasive ductal carcinoma & T4N0M0 & 2 & IIIb & malignant & +++ & - & 0 & + & 108 & + \\
\hline 1258 & 49 & $\mathrm{~F}$ & Breast & nvasive ductal carcinoma (sparse & T4NOMO & 2 & IIIb & malignant & ++ & - & 0 & + & 83 & +++ \\
\hline 1259 & 49 & $\mathrm{~F}$ & Breast & Invasive ductal carcinoma & T4N0M0 & 2 & IIIb & malignant & ++ & - & 0 & + & 75 & +++ \\
\hline 1260 & 69 & $\mathrm{~F}$ & Breast & Invasive ductal carcinoma & T4N0M0 & 2 & IIIb & malignant & ++ & + & 0 & + & 77 & +++ \\
\hline 1261 & 69 & $\mathrm{~F}$ & Breast & Invasive ductal carcinoma & T4N0M0 & 2 & IIIb & malignant & ++ & + & 0 & + & 82 & +++ \\
\hline 1262 & 57 & $\mathrm{~F}$ & Breast & Invasive ductal carcinoma & T4N0M0 & 2 & IIIb & malignant & - & + & 0 & + & 97 & +++ \\
\hline 1263 & 57 & $\mathrm{~F}$ & Breast & Invasive ductal carcinoma & T4NOMO & 2 & IIIb & malignant & + & + & 0 & + & 67 & +++ \\
\hline 1264 & 61 & $\mathrm{~F}$ & Breast & Invasive ductal carcinoma & T4N0MO & 2 & IIlb & malignant & ++ & - & 0 & + & 83 & +++ \\
\hline 1265 & 61 & $\mathrm{~F}$ & Breast & Invasive ductal carcinoma & T4NOMO & 2 & IIlb & malignant & ++ & - & 0 & + & 93 & +++ \\
\hline 1266 & 42 & $\mathrm{~F}$ & Breast & Invasive ductal carcinoma & T4N1M0 & 2 & IIlb & malignant & - & - & 0 & + & 98 & + \\
\hline 1267 & 42 & $\mathrm{~F}$ & Breast & Invasive ductal carcinoma & T4N1M0 & 2 & Illb & malignant & - & - & $1+$ & + & 102 & + \\
\hline 1268 & 52 & $\mathrm{~F}$ & Breast & Invasive ductal carcinoma & T4N1M0 & 2 & IIlb & malignant & + & - & $3+$ & + & 117 & + \\
\hline 1269 & 52 & $\mathrm{~F}$ & Breast & Invasive ductal carcinoma & T4N1M0 & 2 & IIlb & malignant & + & - & $3+$ & + & 126 & + \\
\hline 1270 & 41 & $\mathrm{~F}$ & Breast & Invasive ductal carcinoma & T4NOMO & 2 & IIIb & malignant & ++ & - & 0 & + & 88 & +++ \\
\hline 1271 & 41 & $\mathrm{~F}$ & Breast & Invasive ductal carcinoma & T4NOMO & 2 & IIIb & malignant & + & - & 0 & + & 83 & +++ \\
\hline 1272 & 42 & $\mathrm{~F}$ & Breast & Invasive ductal carcinoma & $\mathrm{T} 4 \mathrm{~N} 2 \mathrm{MO}$ & 2 & IIIb & malignant & ++ & + & 0 & + & 92 & +++ \\
\hline 1273 & 42 & $\mathrm{~F}$ & Breast & asive ductal carcinoma with necrc & $\mathrm{T} 4 \mathrm{~N} 2 \mathrm{M} 0$ & 2 & IIIb & malignant & ++ & + & 0 & + & 106 & +++ \\
\hline 1274 & 53 & $\mathrm{~F}$ & Breast & Invasive ductal carcinoma & $\mathrm{T} 4 \mathrm{~N} 1 \mathrm{M} 0$ & 2 & IIIb & malignant & ++ & * & 0 & + & 94 & +++ \\
\hline 1275 & 53 & $\mathrm{~F}$ & Breast & Invasive ductal carcinoma & $\mathrm{T} 4 \mathrm{~N} 1 \mathrm{M} 0$ & 2 & IIlb & malignant & ++ & * & 0 & + & 81 & +++ \\
\hline 1276 & 60 & $\mathrm{~F}$ & Breast & Invasive ductal carcinoma & $\mathrm{T} 4 \mathrm{~N} 2 \mathrm{M} 0$ & 2 & IIIb & malignant & - & - & 0 & + & 74 & +++ \\
\hline 1277 & 60 & $\mathrm{~F}$ & Breast & Invasive ductal carcinoma & T4N2M0 & 2 & IIIb & malignant & - & - & 0 & + & 66 & +++ \\
\hline 1278 & 52 & $\mathrm{~F}$ & Breast & Invasive ductal carcinoma & T4NOMO & 2 & IIIb & malignant & +++ & - & 0 & + & 58 & +++ \\
\hline 1279 & 52 & $\mathrm{~F}$ & Breast & Invasive ductal carcinoma & T4NOMO & 2 & IIIb & malignant & +++ & - & 0 & + & 87 & +++ \\
\hline 1280 & 49 & $\mathrm{~F}$ & Breast & Invasive ductal carcinoma & $\mathrm{T} 4 \mathrm{~N} 1 \mathrm{M} 0$ & 2 & IIIb & malignant & + & + & 0 & + & 95 & +++ \\
\hline 1281 & 49 & $\mathrm{~F}$ & Breast & Invasive ductal carcinoma & $\mathrm{T} 4 \mathrm{~N} 1 \mathrm{M} 0$ & 2 & IIIb & malignant & + & + & 0 & + & 102 & ++ \\
\hline 1282 & 49 & $\mathrm{~F}$ & Breast & Invasive ductal carcinoma & T4N2M0 & 2 & IIIb & malignant & - & - & 0 & + & 111 & ++ \\
\hline 1283 & 49 & $\mathrm{~F}$ & Breast & Invasive ductal carcinoma & $\mathrm{T} 4 \mathrm{~N} 2 \mathrm{M} 0$ & 2 & IIlb & malignant & - & - & 0 & + & 102 & ++ \\
\hline 1284 & 52 & $\mathrm{~F}$ & Breast & Invasive ductal carcinoma & T4cN2M0 & 2 & IIlb & malignant & - & - & 0 & + & 98 & +++ \\
\hline 1285 & 52 & $\mathrm{~F}$ & Breast & Invasive ductal carcinoma & $\mathrm{T} 4 \mathrm{cN} 2 \mathrm{MO}$ & 2 & Illb & malignant & - & - & 0 & + & 73 & +++ \\
\hline
\end{tabular}




\begin{tabular}{|c|c|c|c|c|c|c|c|c|c|c|c|c|c|c|}
\hline 1286 & 62 & $\mathrm{~F}$ & Breast & Invasive ductal carcinoma & T4N0M0 & 3 & Illb & malignant & - & - & $2+$ & + & 79 & +++ \\
\hline 1287 & 62 & $\mathrm{~F}$ & Breast & Invasive ductal carcinoma & T4NOMO & 3 & IIIb & malignant & - & - & $2+$ & + & 58 & +++ \\
\hline 1288 & 74 & $\mathrm{~F}$ & Breast & Invasive papillary carcinoma & T4NOMO & - & IIIb & malignant & +++ & - & - & + & 52 & +++ \\
\hline 1289 & 74 & $\mathrm{~F}$ & Breast & Invasive papillary carcinoma & T4NOMO & - & IIIb & malignant & +++ & - & - & + & 67 & +++ \\
\hline 1290 & 47 & $\mathrm{~F}$ & Breast & Invasive lobular carcinoma & T4NOMO & - & IIIb & malignant & ++ & ++ & 0 & + & 108 & +++ \\
\hline 1291 & 47 & $\mathrm{~F}$ & Breast & Invasive lobular carcinoma & T4NOMO & - & IIIb & malignant & ++ & ++ & 0 & + & 156 & +++ \\
\hline 1292 & 73 & $\mathrm{~F}$ & Breast & ive mixed lobular and ductal carci & T4N0M0 & - & IIIb & malignant & - & * & 0 & + & 201 & +++ \\
\hline 1293 & 73 & $\mathrm{~F}$ & Breast & ive mixed lobular and ductal carci & T4NOMO & - & IIIb & malignant & - & * & 0 & + & 187 & +++ \\
\hline 1294 & 48 & $\mathrm{~F}$ & Breast & Squamous cell carcinoma & T4N0M0 & $2--3$ & IIIb & malignant & - & - & 0 & + & 184 & + \\
\hline 1295 & 48 & $\mathrm{~F}$ & Breast & Squamous cell carcinoma & T4NOMO & $2--3$ & IIIb & malignant & - & - & 0 & + & 177 & + \\
\hline 1296 & 37 & $\mathrm{~F}$ & Breast & Invasive lobular carcinoma & $\mathrm{T} 4 \mathrm{~N} 1 \mathrm{M} 0$ & & IIIB & malignant & & & & + & 153 & + \\
\hline 1297 & 73 & $\mathrm{~F}$ & Breast & Invasive lobular carcinoma & T4NOMO & & IIIB & malignant & & & & + & 122 & + \\
\hline 1298 & 51 & $\mathrm{~F}$ & Breast & Invasive lobular carcinoma & T4NOMO & & IIIB & malignant & & & & + & 86 & ++ \\
\hline 1299 & 41 & $\mathrm{~F}$ & Breast & Invasive lobular carcinoma & T4NOMO & & IIIB & malignant & & & & + & 66 & ++ \\
\hline 1300 & 53 & $\mathrm{~F}$ & Breast & nvasive lobular carcinoma (spars $€$ & T4N1M0 & & IIIB & malignant & & & & + & 79 & ++ \\
\hline 1301 & 32 & $\mathrm{~F}$ & Breast & Invasive ductal carcinoma & T4N1M0 & 2 & IIIB & Malignant & ++ & - & $3+$ & + & 118 & + \\
\hline 1302 & 60 & $\mathrm{~F}$ & Breast & Invasive ductal carcinoma & T4N2M0 & 2 & IIIB & Malignant & - & - & $3+$ & + & 105 & + \\
\hline 1303 & 32 & $\mathrm{~F}$ & Breast & Invasive ductal carcinoma & T4N1M0 & 2 & IIIB & Malignant & ++ & - & $3+$ & + & 101 & + \\
\hline 1304 & 60 & $\mathrm{~F}$ & Breast & Invasive ductal carcinoma & $\mathrm{T} 4 \mathrm{~N} 2 \mathrm{MO}$ & 2 & IIIB & Malignant & - & - & $3+$ & + & 162 & + \\
\hline 1305 & 44 & $\mathrm{~F}$ & Breast & Invasive ductal carcinoma & $\mathrm{T} 2 \mathrm{~N} 3 \mathrm{M} 0$ & 2 & IV & malignant & - & - & $2+$ & + & 191 & +++ \\
\hline 1306 & 44 & $\mathrm{~F}$ & Breast & Invasive ductal carcinoma & $\mathrm{T} 2 \mathrm{~N} 3 \mathrm{M} 0$ & 2 & IV & malignant & - & - & $2+$ & + & 156 & +++ \\
\hline 1307 & 31 & $\mathrm{~F}$ & Breast & Invasive ductal carcinoma & T4N2M0 & 2 & IV & malignant & +++ & +++ & 0 & + & 153 & +++ \\
\hline 1308 & 31 & $\mathrm{~F}$ & Breast & Invasive ductal carcinoma & $\mathrm{T} 4 \mathrm{~N} 2 \mathrm{M} 0$ & 2 & IV & malignant & +++ & +++ & 0 & + & 126 & +++ \\
\hline 1309 & 57 & $\mathrm{~F}$ & Breast & Invasive ductal carcinoma & $\mathrm{T} 4 \mathrm{~N} 1 \mathrm{M} 0$ & 2 & IV & malignant & - & - & 0 & + & 115 & +++ \\
\hline 1310 & 57 & $\mathrm{~F}$ & Breast & Invasive ductal carcinoma & $\mathrm{T} 4 \mathrm{~N} 1 \mathrm{M} 0$ & 2 & IV & malignant & - & - & 0 & + & 103 & +++ \\
\hline 1311 & 73 & $\mathrm{~F}$ & Breast & arcinoma (chronic inflammation of & $\mathrm{T} 4 \mathrm{~N} 1 \mathrm{M} 1$ & - & IV & malignant & * & * & 0 & + & 108 & +++ \\
\hline 1312 & 73 & $\mathrm{~F}$ & Breast & arcinoma (chronic inflammation of & $\mathrm{T} 4 \mathrm{~N} 1 \mathrm{M} 1$ & - & IV & malignant & * & * & 0 & + & 99 & +++ \\
\hline 1313 & 32 & $\mathrm{~F}$ & Breast & Neuroendocrine carcinoma & $\mathrm{T} 2 \mathrm{~N} 3 \mathrm{M} 0$ & - & IV & malignant & - & + & 0 & + & 126 & +++ \\
\hline 1314 & 32 & $\mathrm{~F}$ & Breast & Neuroendocrine carcinoma & $\mathrm{T} 2 \mathrm{~N} 3 \mathrm{M} 0$ & - & IV & malignant & - & + & 0 & + & 89 & +++ \\
\hline 1315 & 50 & $\mathrm{~F}$ & Breast & Invasive ductal carcinoma & T4N3M1 & $1--2$ & IV & malignant & ++ & * & 0 & + & 72 & +++ \\
\hline 1316 & 50 & $\mathrm{~F}$ & Breast & Invasive ductal carcinoma & T4N3M1 & $1--2$ & IV & malignant & ++ & * & 0 & + & 85 & +++ \\
\hline 1317 & 54 & $\mathrm{~F}$ & Breast & Invasive ductal carcinoma & T3NOMO & $1--2$ & & Malignant & & & & + & 92 & + \\
\hline 1318 & 38 & $\mathrm{~F}$ & Breast & Invasive ductal carcinoma & T3NOMO & $1--2$ & & Malignant & +++ & +++ & $3+$ & + & 101 & + \\
\hline 1319 & 38 & $\mathrm{~F}$ & Breast & Invasive ductal carcinoma & T3N0MO & $1--2$ & & Malignant & +++ & +++ & $3+$ & + & 67 & + \\
\hline
\end{tabular}




$\begin{array}{llll}1320 & 41 & \mathrm{~F} & \text { Breast } \\ 1321 & 41 & \mathrm{~F} & \text { Breast } \\ 1322 & 35 & \mathrm{~F} & \text { Breast } \\ 1323 & 32 & \mathrm{~F} & \text { Breast } \\ 1324 & 38 & \mathrm{~F} & \text { Breast } \\ 1325 & 52 & \mathrm{~F} & \text { Breast } \\ 1326 & 31 & \mathrm{~F} & \text { Breast } \\ 1327 & 30 & \mathrm{~F} & \text { Breast } \\ 1328 & 30 & \mathrm{~F} & \text { Breast } \\ 1329 & 33 & \mathrm{~F} & \text { Breast } \\ 1330 & 33 & \mathrm{~F} & \text { Breast } \\ 1331 & 35 & \mathrm{~F} & \text { Breast } \\ 1332 & 35 & \mathrm{~F} & \text { Breast } \\ 1333 & 44 & \mathrm{~F} & \text { Breast } \\ 1334 & 44 & \mathrm{~F} & \text { Breast } \\ 1335 & 43 & \mathrm{~F} & \text { Breast } \\ 1336 & 43 & \mathrm{~F} & \text { Breast } \\ 1337 & 41 & \mathrm{~F} & \text { Breast } \\ 1338 & 41 & \mathrm{~F} & \text { Breast } \\ 1339 & 59 & \mathrm{~F} & \text { Breast } \\ 1340 & 59 & \mathrm{~F} & \text { Breast } \\ 1341 & 34 & \mathrm{~F} & \text { Breast } \\ 1342 & 34 & \mathrm{~F} & \text { Breast } \\ 1343 & 47 & \mathrm{~F} & \text { Breast } \\ 1344 & 47 & \mathrm{~F} & \text { Breast } \\ 1345 & & \mathrm{~F} & \text { breast } \\ 1346 & & \mathrm{~F} & \text { breast } \\ 1347 & & \mathrm{~F} & \text { breast } \\ 1348 & & \mathrm{~F} & \text { breast } \\ 1349 & & \mathrm{~F} & \text { breast } \\ 1350 & & \mathrm{~F} & \text { breast } \\ 1351 & & \mathrm{~F} & \text { breast } \\ 1352 & & \mathrm{~F} & \text { breast } \\ 1353 & & \mathrm{~F} & \text { breast } \\ & & & \end{array}$

Invasive ductal carcinoma Invasive ductal carcinoma Invasive ductal carcinoma Invasive ductal carcinoma Invasive ductal carcinoma Invasive ductal carcinoma Invasive ductal carcinoma Invasive ductal carcinoma Invasive ductal carcinoma Invasive ductal carcinoma Invasive ductal carcinoma Invasive ductal carcinoma Invasive ductal carcinoma Invasive ductal carcinoma Invasive ductal carcinoma Invasive ductal carcinoma Invasive ductal carcinoma Invasive ductal carcinoma Invasive ductal carcinoma Invasive ductal carcinoma Invasive ductal carcinoma Invasive ductal carcinoma Invasive ductal carcinoma Invasive ductal carcinoma Invasive ductal carcinoma breast tissue breast tissue breast tissue breast tissue breast tissue breast tissue breast tissue breast tissue breast tissue

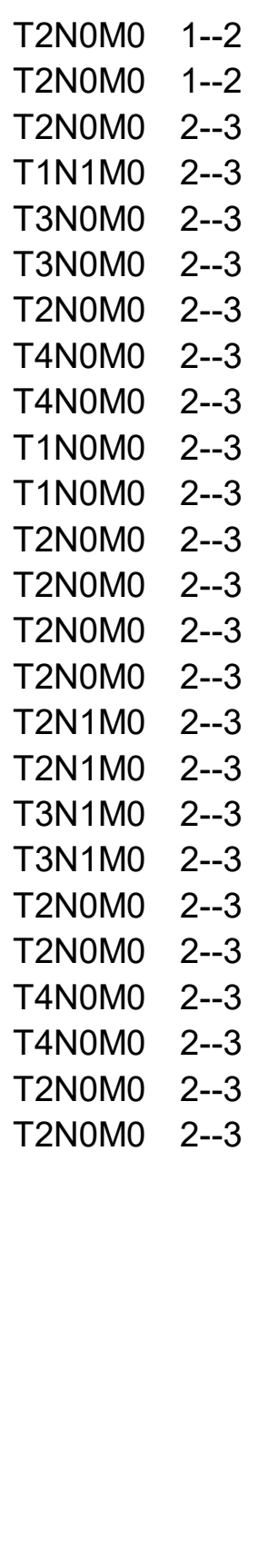

$\begin{array}{cccc}+ & 73 & + & 4 \\ + & 78 & + & 4 \\ + & 82 & + & 4 \\ + & 65 & + & 4 \\ + & 47 & + & 4 \\ + & 58 & + & 4 \\ + & 69 & + & 4 \\ + & 91 & + & 4 \\ + & 107 & + & 4 \\ + & 116 & + & 4 \\ + & 67 & + & 4 \\ + & 73 & + & 4 \\ + & 82 & + & 4 \\ + & 91 & + & 4 \\ + & 103 & + & 4 \\ + & 105 & + & 4 \\ + & 117 & + & 4 \\ + & 88 & + & 4 \\ + & 82 & + & 4 \\ + & 74 & + & 4 \\ + & 109 & + & 4 \\ + & 116 & + & 4 \\ + & 58 & + & 4 \\ + & 78 & + & 4 \\ + & 67 & + & 4 \\ + & 128 & + & 4 \\ + & 115 & + & 4 \\ + & 136 & + & 4 \\ + & 98 & + & 4 \\ + & 176 & + & 4 \\ + & 182 & + & 4 \\ + & 201 & + & 4 \\ + & 153 & + & 4 \\ + & & & 4\end{array}$




\begin{tabular}{|c|c|c|c|c|c|c|c|c|c|c|c|c|c|}
\hline 1354 & & $\mathrm{~F}$ & breast & breast tissue & & & NAT & & & & + & 204 & + \\
\hline 1355 & & $\mathrm{~F}$ & breast & breast tissue & & & NAT & & & & + & 183 & + \\
\hline 1356 & & $F$ & breast & breast tissue & & & NAT & & & & + & 162 & + \\
\hline 1357 & & $\mathrm{~F}$ & breast & breast tissue & & & NAT & & & & + & 188 & + \\
\hline 1358 & & $\mathrm{~F}$ & breast & breast tissue & & & NAT & & & & + & 117 & + \\
\hline 1359 & & $F$ & breast & breast tissue & & & NAT & & & & + & 103 & + \\
\hline 1360 & & $\mathrm{~F}$ & breast & breast tissue & & & NAT & & & & + & 98 & ++ \\
\hline 1361 & & $\mathrm{~F}$ & breast & breast tissue & & & NAT & & & & + & 92 & ++ \\
\hline 1362 & & $F$ & breast & breast tissue & & & NAT & & & & + & 132 & + \\
\hline 1363 & & $F$ & breast & breast tissue & & & NAT & & & & + & 148 & + \\
\hline 1364 & & $F$ & breast & breast tissue & & & NAT & & & & + & 126 & + \\
\hline 1365 & & $F$ & breast & breast tissue & & & NAT & & & & + & 115 & + \\
\hline 1366 & & $F$ & breast & breast tissue & & & NAT & & & & + & 108 & + \\
\hline 1367 & & $\mathrm{~F}$ & breast & breast tissue & & & NAT & & & & + & 101 & + \\
\hline 1368 & & $\mathrm{~F}$ & breast & breast tissue & & & NAT & & & & + & 136 & + \\
\hline 1369 & & $F$ & breast & breast tissue & & & NAT & & & & + & 89 & ++ \\
\hline 1370 & & $\mathrm{~F}$ & breast & breast tissue & & & NAT & & & & + & 96 & ++ \\
\hline 1371 & & $\mathrm{~F}$ & breast & breast tissue & & & NAT & & & & + & 88 & + \\
\hline 1372 & & $\mathrm{~F}$ & breast & breast tissue & & & NAT & & & & + & 102 & + \\
\hline 1373 & & $\mathrm{~F}$ & breast & breast tissue & & & NAT & & & & + & 117 & + \\
\hline 1374 & & $\mathrm{~F}$ & breast & breast tissue & & & NAT & & & & + & 159 & + \\
\hline 1375 & 49 & $\mathrm{~F}$ & Breast & Adjacent normal breast tissue & - & - & NAT & & & & + & 132 & + \\
\hline 1376 & 38 & $\mathrm{~F}$ & Breast & Adjacent normal breast tissue & - & - & NAT & & & & + & 119 & + \\
\hline 1377 & 43 & $\mathrm{~F}$ & Breast & Adjacent normal breast tissue & - & - & NAT & & & & + & 124 & + \\
\hline 1378 & 46 & $\mathrm{~F}$ & Breast & Adjacent normal breast duct tissu€ & - & - & NAT & + & + & 0 & + & 95 & + \\
\hline 1379 & 46 & $\mathrm{~F}$ & Breast & Adjacent normal breast tissue & - & - & NAT & + & + & 0 & + & 105 & + \\
\hline 1380 & 42 & $\mathrm{~F}$ & Breast & Adjacent normal breast tissue & - & - & NAT & - & ++ & 0 & + & 97 & ++ \\
\hline 1381 & 42 & $\mathrm{~F}$ & Breast & :ent normal breast tissue(fibrous ti & - & - & NAT & * & * & * & + & 86 & ++ \\
\hline 1382 & 43 & $\mathrm{~F}$ & Breast & ent normal breast tissue (fibrous $t$ & - & - & NAT & * & * & * & + & 149 & + \\
\hline 1383 & 43 & $\mathrm{~F}$ & Breast & Adjacent normal breast tissue & - & - & NAT & + & + & 0 & + & 133 & + \\
\hline 1384 & 46 & $\mathrm{~F}$ & -ymph noc & Ieatic adenocarcinoma of oxter from & east & 2 & Metastasis & & & & + & 87 & ++ \\
\hline 1385 & 40 & $\mathrm{~F}$ & -ymph noc & I $\operatorname{tatic}$ adenocarcinoma of oxter from & east & 3 & Metastasis & & & & + & 103 & + \\
\hline 1386 & 48 & $\mathrm{~F}$ & -ymph noo & I & east & 2 & Metastasis & & & & + & 114 & + \\
\hline 1387 & 53 & $\mathrm{~F}$ & -ymph noc & Ieatic adenocarcinoma of oxter from & east & 2 & Metastasis & & & & + & 128 & + \\
\hline
\end{tabular}


$138846 \quad F \quad$-ymph nodeatic adenocarcinoma of oxter from breast

$138940 \quad F \quad$-ymph nodeatic adenocarcinoma of oxter from breast

$139053 \quad F \quad$-ymph nod 5 atic adenocarcinoma of oxter from breast

$139142 \quad F$-ymph nodeatic adenocarcinoma of oxter from breast

$139248 \quad F \quad$-ymph nod 4 atic adenocarcinoma of oxter from breast

$139366 \quad M$-ymph nodeatic adenocarcinoma of oxter from breast

$139458 \quad F-y m p h$ nodeatic adenocarcinoma of oxter from breast

$139542 \quad F \quad$-ymph nodeatic adenocarcinoma of oxter from breast

$139666 \quad M$-ymph nod 6 atic adenocarcinoma of oxter from breast

$139758 \quad \mathrm{~F}$-ymph nodєatic adenocarcinoma of oxter from breast

$139870 \mathrm{~F}$ Brain tastasis adenocarcinoma from breast

139943

140055

140134

140234

140375

$1404 \quad 49$

$14054 \varepsilon$

140648

$1407 \quad 15$

14081

14095

14105

1411

1412

1413

1414

1415

China

$141654 \quad \mathrm{~F} \quad$ Liver tastasis adenocarcinoma from breast

$141761 \mathrm{~F}$ Liver tastasis adenocarcinoma from breast

$141852 \mathrm{~F}$ Liver tastasis adenocarcinoma from breast

$141948 \mathrm{~F}$ Lung tastasis adenocarcinoma from breast

$142071 \mathrm{~F}$ Lung tastasis adenocarcinoma from breast
Fibroadenoma

Fibroadenoma

Fibroadenoma

Fibroadenoma

Fibroadenoma

breast tissue

breast tissue

breast tissue

Breast

Breast

F Breast
Metastasis

Metastasis

Metastasis

Metastasis

Metastasis

Metastasis

Metastasis

Metastasis

Metastasis

Metastasis

Metastasis

Metastasis

Metastasis

Metastasis

Metastasis

Metastasis

Metastasis

Benign

Benign

Benign

Benign

Benign

Benign

Normal tissue

Normal tissue

Normal tissue

Normal tissue

Normal tissue

$\begin{array}{cccc}+ & 156 & + & 4 \\ + & 142 & + & 4 \\ + & 108 & + & 4 \\ + & 127 & & 4 \\ + & 114 & & 4 \\ + & 98 & + & 4 \\ + & 107 & + & 4 \\ + & 142 & + & 4 \\ + & 158 & + & 4 \\ + & 192 & & 4 \\ + & 187 & ++ & 4 \\ + & 156 & ++ & 4 \\ + & 192 & + & 4 \\ + & 177 & + & 4 \\ + & 184 & + & 4 \\ + & 179 & ++ & 4 \\ + & 168 & ++ & 4 \\ - & & & 0 \\ - & & & 0 \\ - & & & 0 \\ - & & & 0 \\ - & & & \end{array}$

$\begin{array}{lllllllll}\text { III } & \text { Metastasis } & - & - & - & + & 103 & + & 4 \\ \text { III } & \text { Metastasis } & - & - & - & + & 126 & + & 4 \\ \text { III } & \text { Metastasis } & - & - & - & + & 134 & & 4 \\ \text { III } & \text { Metastasis } & - & - & - & + & 98 & ++ & 4 \\ \text { III } & \text { Metastasis } & - & - & + & + & 107 & ++ & 4\end{array}$

4

4

4

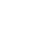

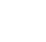
( 4 4 (4) ( 0

-

-

-

- 


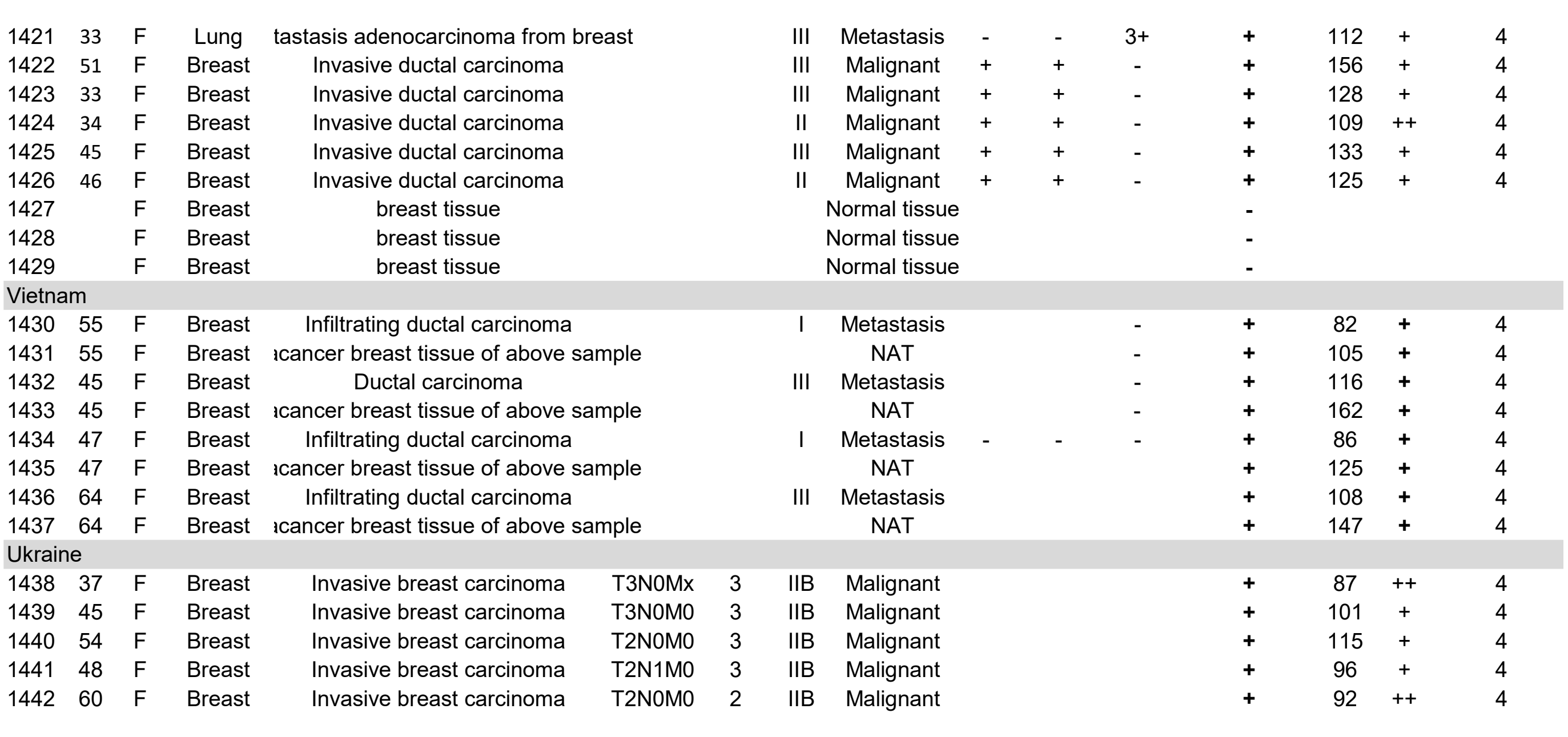




\begin{tabular}{|c|c|c|c|c|c|c|c|c|c|c|c|c|}
\hline No. & Age & Sex & $\begin{array}{l}\text { Organ/ } \\
\text { Anatomic } \\
\text { Site }\end{array}$ & Pathology diagnosis & TNM & Grade & Stage & Type & $\begin{array}{l}\text { Cytocapsular } \\
\text { tubes (CT) }\end{array}$ & $\begin{array}{c}\mathrm{CT} / \\
\mathrm{mm} 2\end{array}$ & $\begin{array}{c}\mathrm{CT} \\
\text { degradation }\end{array}$ & $\begin{array}{c}\text { Cancer } \\
\text { metastasis } \\
\text { grade }\end{array}$ \\
\hline 1 & 49 & M & Colon & Adenocarcinoma & T2NOMO & 1 & I & malignant & + & 23 & + & 2 \\
\hline 2 & 24 & $\mathrm{~F}$ & Colon & Mucinous adenocarcinoma & T2NOMO & 1 & 1 & malignant & + & 18 & + & 2 \\
\hline 3 & 30 & M & Colon & Adenocarcinoma & T1N0M0 & 1 & 1 & malignant & + & 27 & + & 2 \\
\hline 4 & 27 & M & Colon & Adenocarcinoma & T2NOMO & 1 & 1 & malignant & + & 31 & + & 2 \\
\hline 5 & 69 & M & Colon & Adenocarcinoma & T2NOMO & 2 & 1 & malignant & + & 27 & + & 2 \\
\hline 6 & 51 & M & Colon & Mucinous adenocarcinoma & T2NOMO & 1 & 1 & malignant & + & 39 & + & 2 \\
\hline 7 & 68 & M & Colon & Adenocarcinoma & T2NOMO & 1 & I & malignant & + & 43 & + & 3 \\
\hline 8 & 45 & M & Colon & Mucinous adenocarcinoma & T2NOMO & 1 & 1 & malignant & + & 52 & + & 3 \\
\hline 9 & 63 & $\mathrm{~F}$ & Colon & Adenocarcinoma & T2NOMO & 2 & I & malignant & + & 33 & + & 2 \\
\hline 10 & 69 & $\mathrm{~F}$ & Colon & Adenocarcinoma & T2NOMO & 2 & 1 & malignant & + & 29 & + & 2 \\
\hline 11 & 49 & $\mathrm{~F}$ & Colon & Adenocarcinoma & T2NOMO & 2 & I & malignant & + & 46 & + & 3 \\
\hline 12 & 67 & $\mathrm{~F}$ & Colon & Adenocarcinoma & T2NOMO & 1 & I & malignant & + & 31 & + & 2 \\
\hline 13 & 53 & $\mathrm{~F}$ & Colon & Adenocarcinoma & T2NOMO & 2 & 1 & malignant & + & 44 & + & 3 \\
\hline 14 & 69 & M & Colon & Adenocarcinoma & T2NOMO & 2 & 1 & malignant & + & 38 & + & 2 \\
\hline 15 & 64 & M & Colon & Mucinous adenocarcinoma & T2NOMO & 3 & 1 & malignant & + & 52 & + & 3 \\
\hline 16 & 61 & $\mathrm{~F}$ & Colon & Adenocarcinoma & T2NOMO & 2 & 1 & malignant & + & 63 & + & 3 \\
\hline 17 & 70 & $\mathrm{~F}$ & Colon & Mucinous adenocarcinoma & T3NOMO & 1 & I & malignant & + & 46 & + & 3 \\
\hline 18 & 57 & $\mathrm{~F}$ & Colon & Adenocarcinoma & T2NOMO & 2 & 1 & malignant & + & 38 & + & 2 \\
\hline 19 & 43 & $M$ & Colon & Mucinous adenocarcinoma & T2NOMO & 1 & 1 & malignant & + & 29 & + & 2 \\
\hline 20 & 51 & M & Colon & Mucinous adenocarcinoma & T2NOMO & 2 & 1 & malignant & + & 35 & + & 2 \\
\hline 21 & 16 & $M$ & Colon & Adenocarcinoma & T3NOMO & 3 & 1 & malignant & + & 39 & + & 2 \\
\hline 22 & 52 & M & Colon & Mucinous adenocarcinoma & T2NOMO & 3 & I & malignant & + & 45 & + & 3 \\
\hline 23 & 38 & M & Colon & Signet ring cell carcinoma & T3NOMO & - & I & malignant & + & 52 & + & 3 \\
\hline 24 & 50 & M & Colon & Carcinoid & T2NOMO & - & I & malignant & + & 38 & + & 2 \\
\hline 25 & 64 & $\mathrm{~F}$ & Colon & Adenocarcinoma (sparse) & T2NOMO & - & 1 & Malignant & + & 36 & + & 2 \\
\hline 26 & 42 & $\mathrm{~F}$ & Colon & Adenocarcinoma & T2NOMO & 1 & 1 & Malignant & + & 47 & + & 3 \\
\hline 27 & 77 & M & Colon & Adenocarcinoma & T1N0M0 & 1 & 1 & Malignant & + & 53 & + & 3 \\
\hline 28 & 78 & $\mathrm{~F}$ & Colon & Adenocarcinoma & T1N0M0 & 1 & 1 & Malignant & + & 61 & + & 3 \\
\hline 29 & 58 & $\mathrm{~F}$ & Colon & Adenocarcinoma (sparse) & T2NOMO & - & I & Malignant & + & 68 & + & 3 \\
\hline 30 & 31 & M & Colon & Adenocarcinoma & T2NOMO & 2 & 1 & Malignant & + & 49 & + & 3 \\
\hline 31 & 52 & M & Colon & Mucinous adenocarcinoma & T2NOMO & 1 & 1 & Malignant & + & 54 & + & 3 \\
\hline 32 & 36 & M & Colon & Mucinous adenocarcinoma & T1N0M0 & 1 & 1 & Malignant & + & 37 & + & 2 \\
\hline 33 & 55 & $\mathrm{~F}$ & Colon & Mucinous adenocarcinoma & T2NOMO & 3 & 1 & Malignant & + & 66 & + & 3 \\
\hline 34 & 57 & $\mathrm{~F}$ & Colon & Mucinous adenocarcinoma & T2NOMO & 2 & I & Malignant & + & 42 & + & 3 \\
\hline 35 & 44 & $\mathrm{~F}$ & Colon & Mucinous adenocarcinoma & T1N0M0 & 2 & 1 & Malignant & + & 71 & + & 3 \\
\hline 36 & 63 & M & Colon & Adenocarcinoma & T2NOMO & 1 & 1 & malignant & + & 28 & + & 2 \\
\hline
\end{tabular}




\begin{tabular}{|c|c|c|c|c|c|}
\hline 37 & 63 & M & Colon & Adenocarcinoma & T2N0MO \\
\hline 38 & 40 & M & Colon & Adenocarcinoma & T2NOMO \\
\hline 39 & 40 & $\mathrm{M}$ & Colon & Adenocarcinoma & T2NOMO \\
\hline 40 & 66 & M & Colon & Adenocarcinoma & T2N0MO \\
\hline 41 & 66 & M & Colon & Adenocarcinoma & T2N0MO \\
\hline 42 & 51 & $\mathrm{~F}$ & Colon & Adenocarcinoma (chronic inflammatic & T2NOMO \\
\hline 43 & 51 & $\mathrm{~F}$ & Colon & Adenocarcinoma (chronic inflammatic & T2NOMO \\
\hline 44 & 55 & M & Colon & Adenocarcinoma & T2N0MO \\
\hline 45 & 55 & M & Colon & Adenocarcinoma & T2N0MO \\
\hline 46 & 70 & M & Colon & Adenocarcinoma & T2N0MO \\
\hline 47 & 70 & $\mathrm{M}$ & Colon & Adenocarcinoma & T2NOMO \\
\hline 48 & 48 & M & Colon & Adenocarcinoma & T2N0MO \\
\hline 49 & 48 & M & Colon & Adenocarcinoma & T2NOMO \\
\hline 50 & 47 & $\mathrm{~F}$ & Colon & Adenocarcinoma & T2NOMO \\
\hline 51 & 47 & $\mathrm{~F}$ & Colon & Adenocarcinoma & T2NOMO \\
\hline 52 & 66 & $\mathrm{~F}$ & Colon & Adenocarcinoma (chronic inflammatic & T2NOMO \\
\hline 53 & 66 & $\mathrm{~F}$ & Colon & Adenocarcinoma & T2N0MO \\
\hline 54 & 56 & $\mathrm{M}$ & Colon & Adenocarcinoma & T2NOMO \\
\hline 55 & 56 & M & Colon & Adenocarcinoma & T2NOMO \\
\hline 56 & 45 & $\mathrm{~F}$ & Colon & Adenocarcinoma (sparse) & T2N0MO \\
\hline 57 & 45 & $\mathrm{~F}$ & Colon & Adenocarcinoma & T2NOMO \\
\hline 58 & 72 & M & Colon & Adenocarcinoma & T2N0MO \\
\hline 59 & 72 & M & Colon & Adenocarcinoma & T2N0MO \\
\hline 60 & 39 & M & Colon & Adenocarcinoma & T2N0MO \\
\hline 61 & 39 & M & Colon & Adenocarcinoma & T2NOMO \\
\hline 62 & 46 & $\mathrm{~F}$ & Colon & Adenocarcinoma & T2NOMO \\
\hline 63 & 46 & $\mathrm{~F}$ & Colon & Adenocarcinoma & T2N0M0 \\
\hline 64 & 61 & M & Colon & Adenocarcinoma & T2N0MO \\
\hline 65 & 61 & M & Colon & Adenocarcinoma & T2NOMO \\
\hline 66 & 70 & $M$ & Colon & Adenocarcinoma & T2NOMO \\
\hline 67 & 70 & M & Colon & Adenocarcinoma & T2N0MO \\
\hline 68 & 44 & M & Colon & Adenocarcinoma & T2NOMO \\
\hline 69 & 44 & M & Colon & Adenocarcinoma & T2NOMO \\
\hline 70 & 64 & $\mathrm{~F}$ & Colon & Adenocarcinoma & T2NOMO \\
\hline 71 & 64 & $\mathrm{~F}$ & Colon & Adenocarcinoma & T2NOMO \\
\hline 72 & 75 & M & Colon & Adenocarcinoma & T2NOMO \\
\hline 73 & 75 & $\mathrm{M}$ & Colon & Adenocarcinoma & T2NOMO \\
\hline
\end{tabular}

\begin{tabular}{|c|c|c|c|}
\hline malignant & + & 36 & + \\
\hline malignant & + & 41 & + \\
\hline malignant & + & 72 & + \\
\hline malignant & + & 43 & + \\
\hline malignant & + & 36 & + \\
\hline malignant & + & 53 & + \\
\hline malignant & + & 47 & + \\
\hline malignant & + & 38 & + \\
\hline malignant & + & 29 & + \\
\hline malignant & + & 40 & + \\
\hline malignant & + & 53 & + \\
\hline malignant & + & 59 & + \\
\hline malignant & + & 25 & + \\
\hline malignant & + & 32 & + \\
\hline malignant & + & 44 & + \\
\hline malignant & + & 58 & + \\
\hline malignant & + & 75 & + \\
\hline malignant & + & 27 & + \\
\hline malignant & + & 31 & + \\
\hline malignant & + & 33 & + \\
\hline malignant & + & 42 & + \\
\hline malignant & + & 45 & + \\
\hline malignant & + & 39 & + \\
\hline malignant & + & 31 & + \\
\hline malignant & + & 26 & + \\
\hline malignant & + & 34 & + \\
\hline malignant & + & 55 & + \\
\hline malignant & + & 64 & + \\
\hline malignant & + & 57 & + \\
\hline malignant & + & 61 & + \\
\hline malignant & + & 72 & + \\
\hline malignant & + & 43 & + \\
\hline malignant & + & 37 & + \\
\hline malignant & + & 35 & + \\
\hline malignant & + & 43 & + \\
\hline malignant & + & 49 & + \\
\hline malignant & + & 36 & + \\
\hline
\end{tabular}




\begin{tabular}{|c|c|c|c|c|c|c|c|c|c|c|c|}
\hline 74 & 51 & M & Colon & Adenocarcinoma & T2NOMO & 2 & 1 & malignant & + & 40 & + \\
\hline 75 & 51 & M & Colon & Adenocarcinoma & T2NOMO & 2 & 1 & malignant & + & 57 & + \\
\hline 76 & 75 & $\mathrm{~F}$ & Colon & Adenocarcinoma & T2NOMO & 2 & I & malignant & + & 69 & + \\
\hline 77 & 75 & $\mathrm{~F}$ & Colon & Adenocarcinoma & T2NOMO & 2 & 1 & malignant & + & 70 & + \\
\hline 78 & 72 & $\mathrm{~F}$ & Colon & Adenocarcinoma & T2NOMO & 2 & 1 & malignant & + & 54 & + \\
\hline 79 & 72 & $\mathrm{~F}$ & Colon & Adenocarcinoma & T2NOMO & 2 & 1 & malignant & + & 43 & + \\
\hline 80 & 90 & M & Colon & Adenocarcinoma & T2NOMO & 2 & 1 & malignant & + & 37 & + \\
\hline 81 & 90 & $M$ & Colon & Adenocarcinoma & T2NOMO & 2 & 1 & malignant & + & 62 & + \\
\hline 82 & 77 & $\mathrm{~F}$ & Colon & Adenocarcinoma (smooth muscle) & T2NOMO & - & I & malignant & + & 56 & + \\
\hline 83 & 77 & $\mathrm{~F}$ & Colon & Adenocarcinoma & T2NOMO & 2 & 1 & malignant & + & 51 & + \\
\hline 84 & 26 & $\mathrm{~F}$ & Colon & Adenocarcinoma & T2NOMO & 2 & 1 & malignant & + & 48 & + \\
\hline 85 & 26 & $\mathrm{~F}$ & Colon & Adenocarcinoma (colon tissue) & T2NOMO & - & I & malignant & + & 37 & + \\
\hline 86 & 46 & $\mathrm{~F}$ & Colon & Adenocarcinoma & T2NOMO & 2 & 1 & malignant & + & 52 & + \\
\hline 87 & 46 & $\mathrm{~F}$ & Colon & Adenocarcinoma & T2NOMO & 2 & 1 & malignant & + & 27 & + \\
\hline 88 & 64 & M & Colon & Adenocarcinoma & T2NOMO & 2 & 1 & malignant & + & 45 & + \\
\hline 89 & 64 & $M$ & Colon & Adenocarcinoma & T2NOMO & 2 & 1 & malignant & + & 48 & + \\
\hline 90 & 78 & M & Colon & Adenocarcinoma & T2NOMO & 2 & 1 & malignant & + & 53 & + \\
\hline 91 & 78 & M & Colon & Adenocarcinoma & T2NOMO & 2 & 1 & malignant & + & 58 & + \\
\hline 92 & 81 & M & Colon & Adenocarcinoma & T2NOMO & 2 & I & malignant & + & 64 & + \\
\hline 93 & 81 & M & Colon & Adenocarcinoma & T2NOMO & 2 & 1 & malignant & + & 71 & + \\
\hline 94 & 29 & $\mathrm{~F}$ & Colon & Adenocarcinoma & T2NOMO & 2 & 1 & malignant & + & 26 & + \\
\hline 95 & 29 & $\mathrm{~F}$ & Colon & Adenocarcinoma & T2NOMO & 2 & 1 & malignant & + & 35 & + \\
\hline 96 & 57 & M & Colon & Adenocarcinoma & T2NOMO & 2 & 1 & malignant & + & 33 & + \\
\hline 97 & 57 & M & Colon & Adenocarcinoma & T2NOMO & 2 & 1 & malignant & + & 42 & + \\
\hline 98 & 46 & M & Colon & Adenocarcinoma (sparse) & T2N0MO & - & I & malignant & + & 48 & + \\
\hline 99 & 46 & M & Colon & Adenocarcinoma (chronic inflammatic & T2NOMO & - & 1 & malignant & + & 56 & + \\
\hline 100 & 62 & M & Colon & Adenocarcinoma & T2NOMO & 2 & 1 & malignant & + & 43 & + \\
\hline 101 & 62 & $M$ & Colon & Adenocarcinoma & T2NOMO & 2 & 1 & malignant & + & 33 & + \\
\hline 102 & 68 & M & Colon & Adenocarcinoma & T2NOMO & 2 & I & malignant & + & 51 & + \\
\hline 103 & 68 & M & Colon & Adenocarcinoma & T2NOMO & 2 & 1 & malignant & + & 57 & + \\
\hline 104 & 60 & $\mathrm{~F}$ & Colon & Adenocarcinoma & T2N0MO & 2 & 1 & malignant & + & 35 & + \\
\hline 105 & 60 & $F$ & Colon & Adenocarcinoma & T2NOMO & 2 & 1 & malignant & + & 43 & + \\
\hline 106 & 80 & M & Colon & Adenocarcinoma & T2NOMO & 2 & 1 & malignant & + & 36 & + \\
\hline 107 & 80 & M & Colon & Adenocarcinoma & T2NOMO & 2 & 1 & malignant & + & 58 & + \\
\hline 108 & 81 & M & Colon & Adenocarcinoma & T2NOMO & 2 & 1 & malignant & + & 65 & + \\
\hline 109 & 81 & $M$ & Colon & Adenocarcinoma & T2NOMO & 2 & 1 & malignant & + & 45 & + \\
\hline
\end{tabular}




\begin{tabular}{|c|c|c|c|c|c|c|c|c|c|c|c|}
\hline 110 & 58 & $\mathrm{~F}$ & Colon & Adenocarcinoma & T2NOMO & 2 & 1 & malignant & + & 41 & + \\
\hline 111 & 58 & $\mathrm{~F}$ & Colon & Adenocarcinoma & T2NOMO & 2 & 1 & malignant & + & 30 & + \\
\hline 112 & 60 & $\mathrm{~F}$ & Colon & Mucinous adenocarcinoma & T2NOMO & 1 & 1 & malignant & + & 27 & + \\
\hline 113 & 60 & $\mathrm{~F}$ & Colon & Mucinous adenocarcinoma & T2NOMO & 1 & 1 & malignant & + & 23 & + \\
\hline 114 & 62 & $M$ & Colon & Adenocarcinoma & T2NOMO & 2 & I & malignant & + & 33 & + \\
\hline 115 & 62 & M & Colon & Adenocarcinoma & T2NOMO & 2 & 1 & malignant & + & 36 & + \\
\hline 116 & 51 & M & Colon & Adenocarcinoma & T2NOMO & $2--3$ & 1 & malignant & + & 47 & + \\
\hline 117 & 51 & $M$ & Colon & Adenocarcinoma & T2NOMO & $2--3$ & 1 & malignant & + & 51 & + \\
\hline 118 & 80 & $\mathrm{~F}$ & Colon & Adenocarcinoma & T2NOMO & $2--3$ & I & malignant & + & 55 & + \\
\hline 119 & 80 & $\mathrm{~F}$ & Colon & Adenocarcinoma & T2NOMO & $2--3$ & 1 & malignant & + & 63 & + \\
\hline 120 & 56 & $\mathrm{~F}$ & Colon & Adenocarcinoma & T2NOMO & $2--3$ & 1 & malignant & + & 58 & + \\
\hline 121 & 56 & $\mathrm{~F}$ & Colon & Adenocarcinoma & T2NOMO & $2--3$ & 1 & malignant & + & 49 & + \\
\hline 122 & 48 & M & Colon & Adenocarcinoma (sparse) & T2NOMO & 3 & 1 & malignant & + & 32 & + \\
\hline 123 & 48 & M & Colon & Adenocarcinoma & T2NOMO & 3 & 1 & malignant & + & 42 & + \\
\hline 124 & 65 & $\mathrm{~F}$ & Colon & Adenocarcinoma & T2NOMO & 3 & 1 & malignant & + & 47 & + \\
\hline 125 & 65 & $\mathrm{~F}$ & Colon & Adenocarcinoma & T2NOMO & 3 & 1 & malignant & + & 34 & + \\
\hline 126 & 39 & M & Colon & Undifferentiated carcinoma & T2NOMO & - & 1 & malignant & + & 56 & + \\
\hline 127 & 39 & M & Colon & Undifferentiated carcinoma & T2NOMO & - & 1 & malignant & + & 68 & + \\
\hline 128 & 56 & M & Colon & Adenocarcinoma & T3NOMO & 1 & II & malignant & + & 83 & + \\
\hline 129 & 56 & $\mathrm{~F}$ & Colon & Adenocarcinoma & T3NOMO & 1 & II & malignant & + & 92 & + \\
\hline 130 & 41 & $\mathrm{~F}$ & Colon & Adenocarcinoma & T3NOMO & 1 & II & malignant & + & 83 & + \\
\hline 131 & 61 & M & Colon & Adenocarcinoma & T4NOMO & 1 & II & malignant & + & 76 & + \\
\hline 132 & 40 & M & Colon & Adenocarcinoma & T3NOMO & 1 & II & malignant & + & 69 & + \\
\hline 133 & 64 & M & Colon & Adenocarcinoma & T4NOMO & 1 & II & malignant & + & 84 & + \\
\hline 134 & 73 & $\mathrm{~F}$ & Colon & Adenocarcinoma & T4NOMO & 1 & II & malignant & + & 81 & + \\
\hline 135 & 69 & M & Colon & Adenocarcinoma & T4NOMO & 1 & II & malignant & + & 94 & + \\
\hline 136 & 64 & M & Colon & Adenocarcinoma(fibrous tissue) & T4NOMO & - & II & malignant & + & 87 & + \\
\hline 137 & 51 & $\mathrm{~F}$ & Colon & Adenocarcinoma & T4NOMO & 2 & II & malignant & + & 76 & + \\
\hline 138 & 75 & M & Colon & Adenocarcinoma & T3NOMO & 1 & II & malignant & + & 96 & + \\
\hline 139 & 59 & M & Colon & Mucinous adenocarcinoma & T4NOMO & 1 & II & malignant & + & 102 & + \\
\hline 140 & 73 & M & Colon & Adenocarcinoma & T3NOMO & 2 & II & malignant & + & 113 & + \\
\hline 141 & 53 & M & Colon & Adenocarcinoma(colonic mucosa tiss & T3NOMO & - & II & malignant & + & 98 & + \\
\hline 142 & 50 & M & Colon & Adenocarcinoma & T3NOMO & 1 & II & malignant & + & 84 & + \\
\hline 143 & 38 & $\mathrm{~F}$ & Colon & Adenocarcinoma & T3NOMO & 1 & II & malignant & + & 87 & + \\
\hline 144 & 51 & $\mathrm{~F}$ & Colon & Adenocarcinoma & T3NOMO & 1 & II & malignant & + & 93 & + \\
\hline 145 & 46 & $M$ & Colon & Adenocarcinoma & T3NOMO & 1 & II & malignant & + & 103 & + \\
\hline
\end{tabular}




\begin{tabular}{|c|c|c|c|c|c|c|c|c|c|c|c|c|}
\hline 146 & 40 & $\mathrm{~F}$ & Colon & Mucinous adenocarcinoma & T3NOMO & 1 & II & malignant & + & 108 & + & 4 \\
\hline 147 & 50 & M & Colon & Mucinous adenocarcinoma & T3NOMO & 1 & II & malignant & + & 99 & + & 4 \\
\hline 148 & 44 & $\mathrm{~F}$ & Colon & Adenocarcinoma & T3NOMO & 1 & II & malignant & + & 87 & + & 4 \\
\hline 149 & 54 & M & Colon & Adenocarcinoma & T3NOMO & 2 & II & malignant & + & 92 & + & 4 \\
\hline 150 & 45 & M & Colon & Adenocarcinoma & T3NOMO & 1 & II & malignant & + & 84 & + & 4 \\
\hline 151 & 73 & $\mathrm{~F}$ & Colon & Adenocarcinoma & T4NOMO & 2 & II & malignant & + & 86 & + & 4 \\
\hline 152 & 67 & $\mathrm{~F}$ & Colon & Adenocarcinoma & T3NOMO & 2 & II & malignant & + & 94 & + & 4 \\
\hline 153 & 41 & M & Colon & Adenocarcinoma & T4NOMO & 2 & II & malignant & + & 106 & + & 4 \\
\hline 154 & 62 & M & Colon & Adenocarcinoma & T3NOMO & 1 & II & malignant & + & 115 & + & 4 \\
\hline 155 & 73 & M & Colon & Adenocarcinoma & T3NOMO & 1 & II & malignant & + & 97 & + & 4 \\
\hline 156 & 74 & $\mathrm{~F}$ & Colon & Mucinous adenocarcinoma & T3N0M0 & 1 & II & malignant & + & 89 & + & 4 \\
\hline 157 & 39 & M & Colon & Adenocarcinoma & T3NOMO & 2 & II & malignant & + & 82 & + & 4 \\
\hline 158 & 68 & M & Colon & Adenocarcinoma & T3NOMO & 2 & II & malignant & + & 78 & + & 3 \\
\hline 159 & 76 & M & Colon & Adenocarcinoma & T3NOMO & 2 & II & malignant & + & 73 & + & 3 \\
\hline 160 & 73 & M & Colon & Mucinous adenocarcinoma & T4NOMO & 2 & II & malignant & + & 84 & + & 4 \\
\hline 161 & 62 & $\mathrm{~F}$ & Colon & Adenocarcinoma(sparse) & T4NOMO & 2 & II & malignant & + & 91 & + & 4 \\
\hline 162 & 51 & $M$ & Colon & Adenocarcinoma & T3NOMO & 2 & II & malignant & + & 104 & + & 4 \\
\hline 163 & 55 & $\mathrm{~F}$ & Colon & Adenocarcinoma & T4NOMO & 2 & II & malignant & + & 118 & + & 4 \\
\hline 164 & 48 & $\mathrm{~F}$ & Colon & Adenocarcinoma & T3NOMO & 2 & II & malignant & + & 82 & + & 4 \\
\hline 165 & 40 & $M$ & Colon & Adenocarcinoma & T4NOMO & 2 & II & malignant & + & 91 & + & 4 \\
\hline 166 & 72 & M & Colon & Adenocarcinoma & T4NOMO & 2 & II & malignant & + & 86 & + & 4 \\
\hline 167 & 59 & M & Colon & Adenocarcinoma & T4NOMO & 2 & II & malignant & + & 93 & + & 4 \\
\hline 168 & 53 & M & Colon & Adenocarcinoma & T4NOMO & 2 & II & malignant & + & 97 & + & 4 \\
\hline 169 & 50 & M & Colon & Adenocarcinoma & T4NOMO & 2 & II & malignant & + & 105 & + & 4 \\
\hline 170 & 50 & $M$ & Colon & Adenocarcinoma & T3NOMO & 2 & II & malignant & + & 116 & + & 4 \\
\hline 171 & 70 & $\mathrm{~F}$ & Colon & Adenocarcinoma & T3NOMO & 2 & II & malignant & + & 108 & + & 4 \\
\hline 172 & 53 & $\mathrm{~F}$ & Colon & Adenocarcinoma & T3NOMO & 2 & II & malignant & + & 96 & + & 4 \\
\hline 173 & 63 & $\mathrm{~F}$ & Colon & Adenocarcinoma & T3NOMO & 2 & II & malignant & + & 103 & + & 4 \\
\hline 174 & 41 & M & Colon & Adenocarcinoma & T4NOMO & 1 & II & malignant & + & 126 & + & 4 \\
\hline 175 & 53 & M & Colon & Adenocarcinoma & T3NOMO & 2 & II & malignant & + & 117 & + & 4 \\
\hline 176 & 62 & M & Colon & Adenocarcinoma & T4NOMO & 2 & II & malignant & + & 132 & + & 4 \\
\hline 177 & 50 & M & Colon & Mucinous adenocarcinoma & T3NOMO & 1 & II & malignant & + & 105 & + & 4 \\
\hline 178 & 26 & $\mathrm{~F}$ & Colon & Adenocarcinoma & T3NOMO & 2 & II & malignant & + & 112 & + & 4 \\
\hline 179 & 70 & $\mathrm{~F}$ & Colon & Adenocarcinoma & T3NOMO & 2 & II & malignant & + & 94 & + & 4 \\
\hline 180 & 69 & M & Colon & Adenocarcinoma(fibrous tissue) & T3NOMO & - & II & malignant & + & 96 & + & 4 \\
\hline 181 & 49 & M & Colon & Adenocarcinoma & T3NOMO & 2 & II & malignant & + & 89 & + & 4 \\
\hline
\end{tabular}




\begin{tabular}{|c|c|c|c|c|c|c|c|c|c|c|c|c|}
\hline 182 & 78 & $\mathrm{~F}$ & Colon & Adenocarcinoma & T4NOMO & 2 & II & malignant & + & 86 & + & 4 \\
\hline 183 & 67 & $\mathrm{~F}$ & Colon & Adenocarcinoma & T3NOMO & 2 & II & malignant & + & 98 & + & 4 \\
\hline 184 & 55 & $\mathrm{~F}$ & Colon & Adenocarcinoma & T3NOMO & 2 & II & malignant & + & 102 & + & 4 \\
\hline 185 & 31 & M & Colon & Adenocarcinoma & T4N0M0 & 2 & II & malignant & + & 115 & + & 4 \\
\hline 186 & 64 & $\mathrm{~F}$ & Colon & Adenocarcinoma & T3NOMO & 2 & ॥ & malignant & + & 84 & + & 4 \\
\hline 187 & 62 & M & Colon & Adenocarcinoma & T4N0M0 & 2 & II & malignant & + & 128 & + & 4 \\
\hline 188 & 65 & M & Colon & Adenocarcinoma & T3NOMO & 2 & ॥ & malignant & + & 132 & + & 4 \\
\hline 189 & 71 & M & Colon & Adenocarcinoma & T4NOMO & 2 & II & malignant & + & 84 & + & 4 \\
\hline 190 & 66 & $\mathrm{~F}$ & Colon & Adenocarcinoma & T4NOMO & 2 & II & malignant & + & 90 & + & 4 \\
\hline 191 & 41 & $M$ & Colon & Adenocarcinoma & T3N0M0 & 2 & II & malignant & + & 105 & + & 4 \\
\hline 192 & 53 & $\mathrm{~F}$ & Colon & Adenocarcinoma & T3NOMO & 2 & II & malignant & + & 101 & + & 4 \\
\hline 193 & 53 & M & Colon & Adenocarcinoma & T3NOMO & 2 & ॥ & malignant & + & 93 & + & 4 \\
\hline 194 & 62 & M & Colon & Adenocarcinoma & T3NOMO & 2 & II & malignant & + & 97 & + & 4 \\
\hline 195 & 69 & $\mathrm{~F}$ & Colon & Adenocarcinoma & T3NOMO & 2 & II & malignant & + & 117 & + & 4 \\
\hline 196 & 42 & $\mathrm{~F}$ & Colon & Adenocarcinoma & T3NOMO & 2 & II & malignant & + & 86 & + & 4 \\
\hline 197 & 68 & M & Colon & Adenocarcinoma & T4N0M0 & 2 & II & malignant & + & 93 & + & 4 \\
\hline 198 & 30 & M & Colon & Adenocarcinoma & T4NOMO & 3 & II & malignant & + & 117 & + & 4 \\
\hline 199 & 82 & M & Colon & Adenocarcinoma(fibrous tissue ) & T4NOMO & - & II & malignant & + & 103 & + & 4 \\
\hline 200 & 71 & M & Colon & Adenocarcinoma & T3NOMO & 2 & II & malignant & + & 107 & + & 4 \\
\hline 201 & 65 & M & Colon & Adenocarcinoma & T4N0M0 & 2 & II & malignant & + & 96 & + & 4 \\
\hline 202 & 53 & M & Colon & Adenocarcinoma & T3N0M0 & 2 & II & malignant & + & 127 & + & 4 \\
\hline 203 & 57 & M & Colon & Adenocarcinoma & T4NOMO & 2 & II & malignant & + & 115 & + & 4 \\
\hline 204 & 50 & $\mathrm{~F}$ & Colon & Adenocarcinoma & T3NOMO & 2 & II & malignant & + & 103 & + & 4 \\
\hline 205 & 78 & M & Colon & Adenocarcinoma & T3NOMO & 3 & II & malignant & + & 92 & + & 4 \\
\hline 206 & 49 & M & Colon & Mucinous adenocarcinoma & T3N0M0 & 2 & II & malignant & + & 86 & + & 4 \\
\hline 207 & 70 & $\mathrm{~F}$ & Colon & Mucinous adenocarcinoma & T3NOMO & 2 & II & malignant & + & 79 & ++ & 4 \\
\hline 208 & 34 & $\mathrm{~F}$ & Colon & Adenocarcinoma & T4NOMO & 2 & II & malignant & + & 83 & ++ & 4 \\
\hline 209 & 47 & $\mathrm{~F}$ & Colon & Adenocarcinoma & T3NOMO & 2 & II & malignant & + & 94 & + & 4 \\
\hline 210 & 34 & M & Colon & Adenocarcinoma & T3NOMO & 2 & II & malignant & + & 102 & + & 4 \\
\hline 211 & 55 & M & Colon & Adenocarcinoma & T3NOMO & 3 & II & malignant & + & 114 & + & 4 \\
\hline 212 & 73 & $\mathrm{~F}$ & Colon & Adenocarcinoma & T3NOMO & 3 & II & malignant & + & 148 & + & 4 \\
\hline 213 & 51 & M & Colon & Adenocarcinoma & T4N0M0 & 3 & II & malignant & + & 96 & + & 4 \\
\hline 214 & 76 & $\mathrm{~F}$ & Colon & Adenocarcinoma & T3NOMO & 3 & II & malignant & + & 93 & + & 4 \\
\hline 215 & 67 & $\mathrm{~F}$ & Colon & Adenocarcinoma & T3NOMO & 2 & II & malignant & + & 88 & + & 4 \\
\hline 216 & 51 & $M$ & Colon & Adenocarcinoma & T3NOMO & 3 & II & malignant & + & 109 & + & 4 \\
\hline 217 & 72 & M & Colon & Adenocarcinoma & T3NOMO & 3 & II & malignant & + & 112 & + & 4 \\
\hline
\end{tabular}




\begin{tabular}{|c|c|c|c|c|c|c|c|c|c|c|c|}
\hline 218 & 42 & $\mathrm{~F}$ & Colon & Adenocarcinoma & T3N0MO & 3 & II & malignant & + & 125 & + \\
\hline 219 & 64 & M & Colon & Adenocarcinoma & T3NOMO & 3 & II & malignant & + & 132 & + \\
\hline 220 & 38 & $M$ & Colon & Adenocarcinoma & T4N0M0 & 3 & II & malignant & + & 85 & + \\
\hline 221 & 60 & $\mathrm{~F}$ & Colon & Adenocarcinoma & T3NOMO & 3 & II & malignant & + & 94 & + \\
\hline 222 & 48 & $\mathrm{~F}$ & Colon & Adenocarcinoma & T3NOMO & 3 & II & malignant & + & 90 & + \\
\hline 223 & 39 & $M$ & Colon & Adenocarcinoma & T3N0MO & 3 & II & malignant & + & 84 & + \\
\hline 224 & 74 & $\mathrm{~F}$ & Colon & Adenocarcinoma & T3NOMO & 3 & II & malignant & + & 126 & + \\
\hline 225 & 53 & M & Colon & Adenocarcinoma & T4NOMO & 3 & II & malignant & + & 107 & + \\
\hline 226 & 46 & M & Colon & Mucinous adenocarcinoma & T3NOMO & 3 & ॥ & malignant & + & 118 & + \\
\hline 227 & 49 & $\mathrm{~F}$ & Colon & Mucinous adenocarcinoma & T3N0MO & 2 & II & malignant & + & 98 & + \\
\hline 228 & 51 & $\mathrm{~F}$ & Colon & Mucinous adenocarcinoma & T3NOMO & 2 & ॥ & malignant & + & 101 & + \\
\hline 229 & 41 & M & Colon & Adenocarcinoma & T4NOMO & 3 & II & malignant & + & 88 & + \\
\hline 230 & 35 & M & Colon & Mucinous adenocarcinoma & T4NOMO & 2 & II & malignant & + & 83 & ++ \\
\hline 231 & 24 & M & Colon & Mucinous adenocarcinoma(colonic $\mathrm{m}$ & T3NOMO & - & II & malignant & + & 103 & + \\
\hline 232 & 53 & $\mathrm{~F}$ & Colon & Adenocarcinoma & T4NOMO & 2 & II & malignant & + & 116 & + \\
\hline 233 & 72 & M & Colon & Mucinous adenocarcinoma(colonic $\mathrm{m}$ & T3NOMO & - & ॥ & malignant & + & 108 & + \\
\hline 234 & 58 & $\mathrm{~F}$ & Colon & Signet ring cell carcinoma & T3NOMO & - & ॥ & malignant & + & 127 & + \\
\hline 235 & 42 & M & Colon & Signet ring cell carcinoma & T3NOMO & - & II & malignant & + & 123 & + \\
\hline 236 & 49 & $\mathrm{~F}$ & Colon & Mucinous adenocarcinoma & T3NOMO & 2 & II & malignant & + & 102 & + \\
\hline 237 & 49 & $\mathrm{~F}$ & Colon & Adenocarcinoma & T4NOMO & 3 & II & malignant & + & 93 & + \\
\hline 238 & 35 & M & Colon & Adenocarcinoma & T4NOMO & 3 & ॥ & malignant & + & 97 & + \\
\hline 239 & 62 & M & Colon & Adenocarcinoma & T4NOMO & 3 & II & malignant & + & 86 & + \\
\hline 240 & 59 & M & Colon & Adenosquamous carcinoma & T4NOMO & - & II & malignant & + & 124 & + \\
\hline 241 & 59 & M & Colon & Adenosquamous carcinoma & T3NOMO & - & II & malignant & + & 117 & + \\
\hline 242 & 67 & $\mathrm{~F}$ & Colon & Adenosquamous carcinoma & T3NOMO & - & II & malignant & + & 102 & + \\
\hline 243 & 37 & $\mathrm{~F}$ & Colon & Signet ring cell carcinoma & T3NOMO & - & II & malignant & + & 99 & + \\
\hline 244 & 71 & M & Colon & Signet ring cell carcinoma & T4NOMO & - & ॥ & malignant & + & 102 & + \\
\hline 245 & 35 & $\mathrm{~F}$ & Colon & Atypical carcinoid & T4NOMO & - & II & malignant & + & 104 & + \\
\hline 246 & 27 & M & Colon & Adenocarcinoma & T4NOMO & 1 & II & Malignant & + & 116 & + \\
\hline 247 & 43 & M & Colon & Adenocarcinoma & T3NOMO & 1 & II & Malignant & + & 83 & + \\
\hline 248 & 41 & M & Colon & Adenocarcinoma & T3NOMO & 1 & ॥ & Malignant & + & 86 & + \\
\hline 249 & 54 & M & Colon & Adenocarcinoma & T3N0M0 & 1 & II & Malignant & + & 81 & + \\
\hline 250 & 67 & $\mathrm{~F}$ & Colon & Adenocarcinoma (smooth muscle) & T3NOMO & - & II & Malignant & + & 94 & + \\
\hline 251 & 43 & M & Colon & Adenocarcinoma & T3NOMO & 1 & II & Malignant & + & 92 & + \\
\hline 252 & 73 & $\mathrm{~F}$ & Colon & Adenocarcinoma & T3NOMO & 2 & II & Malignant & + & 97 & + \\
\hline 253 & 53 & $\mathrm{~F}$ & Colon & Adenocarcinoma & T3NOMO & 1 & II & Malignant & + & 87 & + \\
\hline
\end{tabular}




\begin{tabular}{|c|c|c|c|c|c|c|c|c|c|c|c|c|}
\hline 254 & 45 & M & Colon & Mucinous adenocarcinoma & T3NOMO & $1--2$ & II & Malignant & + & 85 & + & 4 \\
\hline 255 & 51 & M & Colon & Adenocarcinoma & T3NOMO & 1 & II & Malignant & + & 103 & + & 4 \\
\hline 256 & 60 & $\mathrm{~F}$ & Colon & Adenocarcinoma & T4NOMO & 1 & II & Malignant & + & 119 & + & 4 \\
\hline 257 & 68 & M & Colon & Adenocarcinoma & T4N0M0 & 1 & II & Malignant & + & 96 & + & 4 \\
\hline 258 & 48 & $\mathrm{M}$ & Colon & Adenocarcinoma & T3NOMO & 1 & II & Malignant & + & 109 & + & 4 \\
\hline 259 & 60 & $\mathrm{~F}$ & Colon & Adenocarcinoma & T3NOMO & 1 & II & Malignant & + & 92 & + & 4 \\
\hline 260 & 75 & $\mathrm{~F}$ & Colon & Adenocarcinoma & T3NOMO & 1 & II & Malignant & + & 87 & + & 4 \\
\hline 261 & 64 & $\mathrm{~F}$ & Colon & Adenocarcinoma & T3NOMO & 1 & II & Malignant & + & 102 & + & 4 \\
\hline 262 & 68 & $M$ & Colon & Adenocarcinoma & T3N0M0 & 1 & II & Malignant & + & 82 & ++ & 4 \\
\hline 263 & 46 & $\mathrm{~F}$ & Colon & Adenocarcinoma & T3NOMO & 1 & II & Malignant & + & 81 & ++ & 4 \\
\hline 264 & 43 & $M$ & Colon & Adenocarcinoma & T4NOMO & 1 & II & Malignant & + & 80 & + & 4 \\
\hline 265 & 38 & $M$ & Colon & Adenocarcinoma & T3NOMO & 1 & II & Malignant & + & 93 & + & 4 \\
\hline 266 & 49 & $\mathrm{~F}$ & Colon & Adenocarcinoma & T3NOMO & 1 & II & Malignant & + & 94 & + & 4 \\
\hline 267 & 71 & M & Colon & Adenocarcinoma & T3NOMO & 1 & II & Malignant & + & 91 & + & 4 \\
\hline 268 & 46 & M & Colon & Adenocarcinoma & T4NOMO & 1 & II & Malignant & + & 87 & + & 4 \\
\hline 269 & 49 & $M$ & Colon & Adenocarcinoma & T4NOMO & 1 & II & Malignant & + & 84 & + & 4 \\
\hline 270 & 70 & M & Colon & Adenocarcinoma & T4NOMO & 1 & II & Malignant & + & 89 & + & 4 \\
\hline 271 & 53 & $\mathrm{~F}$ & Colon & Adenocarcinoma & T3NOMO & 1 & II & Malignant & + & 86 & + & 4 \\
\hline 272 & 43 & M & Colon & Adenocarcinoma (sparse) & T3NOMO & 1 & II & Malignant & + & 93 & + & 4 \\
\hline 273 & 52 & $\mathrm{~F}$ & Colon & Adenocarcinoma (fibrous tissue and I & I T3NOMO & - & II & Malignant & + & 98 & + & 4 \\
\hline 274 & 69 & M & Colon & Mucinous adenocarcinoma & T3NOMO & $1--2$ & II & Malignant & + & 104 & + & 4 \\
\hline 275 & 34 & M & Colon & Mucinous adenocarcinoma & T3NOMO & 1 & II & Malignant & + & 115 & + & 4 \\
\hline 276 & 36 & $\mathrm{~F}$ & Colon & Adenocarcinoma (smooth muscle) & T3NOMO & - & II & Malignant & + & 97 & + & 4 \\
\hline 277 & 65 & $\mathrm{~F}$ & Colon & Adenocarcinoma & T3NOMO & 1 & II & Malignant & + & 93 & + & 4 \\
\hline 278 & 62 & M & Colon & Adenocarcinoma & T3NOMO & 1 & II & Malignant & + & 87 & + & 4 \\
\hline 279 & 69 & M & Colon & Mucinous adenocarcinoma & T3NOMO & 1 & II & Malignant & + & 89 & + & 4 \\
\hline 280 & 65 & M & Colon & Mucinous adenocarcinoma & T3NOMO & 1 & II & Malignant & + & 103 & + & 4 \\
\hline 281 & 56 & M & Colon & Adenocarcinoma (sparse) with necro: & T3NOMO & 1 & II & Malignant & + & 112 & + & 4 \\
\hline 282 & 40 & M & Colon & Adenocarcinoma (sparse) & T3NOMO & 2 & II & Malignant & + & 109 & + & 4 \\
\hline 283 & 53 & $\mathrm{~F}$ & Colon & Mucinous adenocarcinoma & T3NOMO & 1 & II & Malignant & + & 94 & + & 4 \\
\hline 284 & 66 & M & Colon & Adenocarcinoma & T3N0M0 & 2 & II & Malignant & + & 97 & + & 4 \\
\hline 285 & 50 & $\mathrm{~F}$ & Colon & Adenocarcinoma & T3NOMO & 1 & II & Malignant & + & 84 & + & 4 \\
\hline 286 & 63 & $\mathrm{~F}$ & Colon & Adenocarcinoma & T3NOMO & 3 & II & Malignant & + & 87 & ++ & 4 \\
\hline 287 & 64 & $\mathrm{~F}$ & Colon & Adenocarcinoma & T4NOMO & 1 & II & Malignant & + & 81 & ++ & 4 \\
\hline 288 & 32 & M & Colon & Mucinous adenocarcinoma & T3NOMO & $1--2$ & II & Malignant & + & 92 & + & 4 \\
\hline 289 & 68 & $\mathrm{~F}$ & Colon & Adenocarcinoma & T3NOMO & 2 & II & Malignant & + & 98 & + & 4 \\
\hline
\end{tabular}




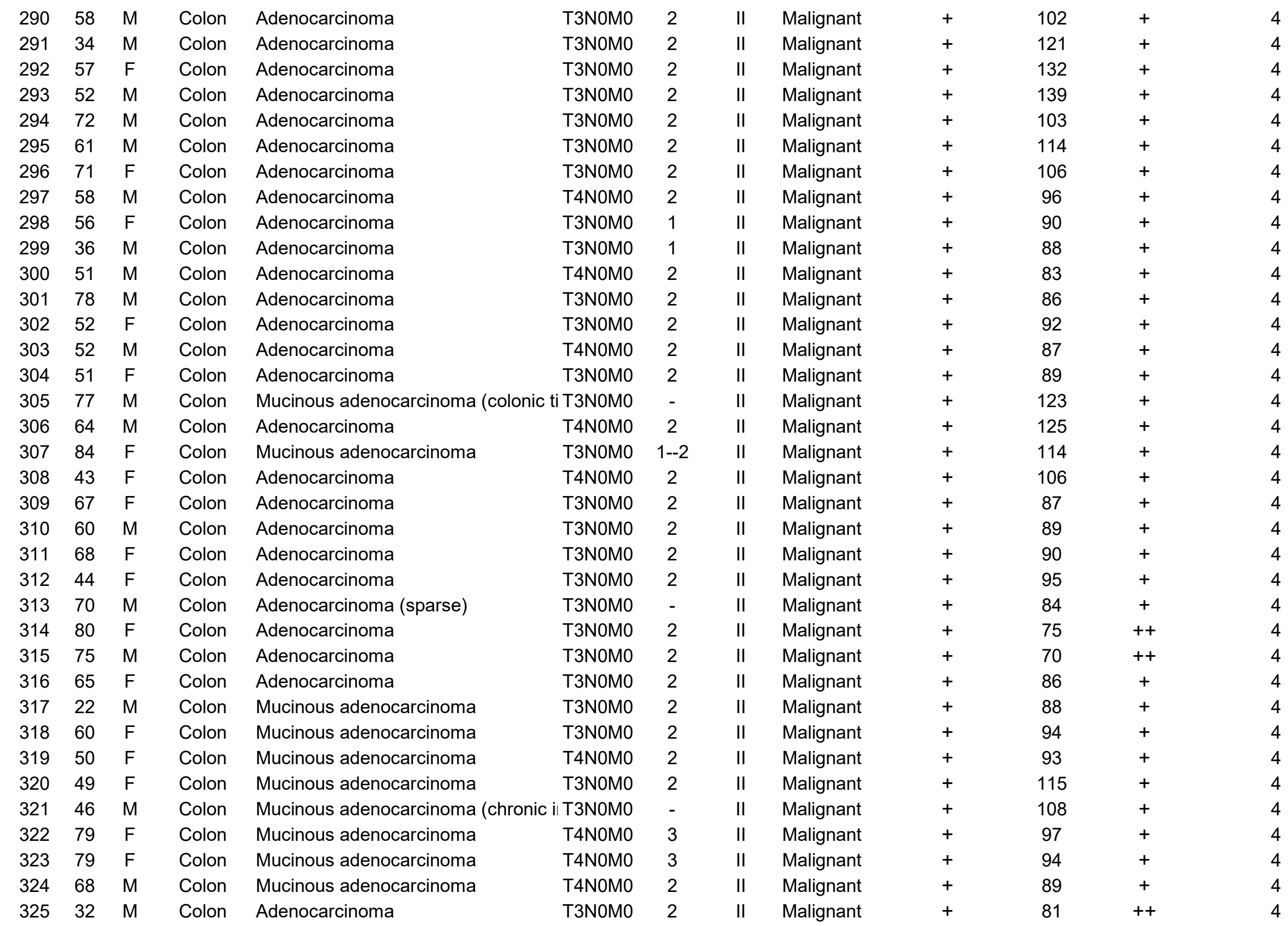




\begin{tabular}{|c|c|c|c|c|c|c|c|c|c|c|c|}
\hline 326 & 30 & $\mathrm{~F}$ & Colon & Mucinous adenocarcinoma & T3NOMO & 3 & II & Malignant & + & 88 & + \\
\hline 327 & 28 & M & Colon & Mucinous adenocarcinoma & T3NOMO & 3 & II & Malignant & + & 83 & + \\
\hline 328 & 47 & M & Colon & Mucinous adenocarcinoma & T4NOMO & 2 & II & Malignant & + & 91 & + \\
\hline 329 & 58 & M & Colon & Adenocarcinoma & T3NOMO & 3 & II & Malignant & + & 118 & + \\
\hline 330 & 82 & $\mathrm{~F}$ & Colon & Adenocarcinoma & T4NOMO & 3 & II & Malignant & + & 103 & + \\
\hline 331 & 58 & $\mathrm{~F}$ & Colon & Adenocarcinoma (fibrous tissue and I & T3NOMO & - & II & Malignant & + & 105 & + \\
\hline 332 & 47 & $\mathrm{~F}$ & Colon & Adenocarcinoma & T3NOMO & 3 & II & Malignant & + & 76 & + \\
\hline 333 & 46 & M & Colon & Adenocarcinoma & T3N0M0 & 3 & ॥ & Malignant & + & 93 & + \\
\hline 334 & 40 & $\mathrm{~F}$ & Colon & Adenocarcinoma & T3NOMO & 3 & II & Malignant & + & 89 & + \\
\hline 335 & 74 & M & Colon & Adenocarcinoma & T3NOMO & 3 & II & Malignant & + & 86 & + \\
\hline 336 & 49 & $M$ & Colon & Adenocarcinoma & T4N0MO & 3 & II & Malignant & + & 84 & + \\
\hline 337 & 33 & M & Colon & Adenocarcinoma & T3NOMO & 3 & II & Malignant & + & 85 & + \\
\hline 338 & 38 & M & Colon & Adenocarcinoma & T3NOMO & 3 & II & Malignant & + & 87 & + \\
\hline 339 & 33 & M & Colon & Adenocarcinoma & T4NOMO & 3 & II & Malignant & + & 96 & + \\
\hline 340 & 31 & M & Colon & Adenocarcinoma & T3NOMO & 3 & II & Malignant & + & 92 & + \\
\hline 341 & 70 & $\mathrm{~F}$ & Colon & Adenocarcinoma & T3NOMO & 3 & II & Malignant & + & 84 & ++ \\
\hline 342 & 54 & M & Colon & Adenocarcinoma (fibrofatty tissue) & T3NOMO & - & II & Malignant & + & 103 & + \\
\hline 343 & 32 & M & Colon & Mucinous adenocarcinoma & T4NOMO & 3 & II & Malignant & + & 115 & + \\
\hline 344 & 33 & $\mathrm{~F}$ & Colon & Mucinous adenocarcinoma & T4N0M0 & 3 & II & Malignant & + & 153 & + \\
\hline 345 & 60 & $\mathrm{~F}$ & Colon & Signet ring cell carcinoma & T4NOMO & - & II & Malignant & + & 126 & + \\
\hline 346 & 45 & M & Colon & Mucinous adenocarcinoma & T3NOMO & 3 & II & Malignant & + & 124 & + \\
\hline 347 & 50 & M & Colon & Signet ring cell carcinoma & T3NOMO & - & II & Malignant & + & 98 & + \\
\hline 348 & 75 & $\mathrm{~F}$ & Colon & Signet ring cell carcinoma & T3NOMO & - & ॥ & Malignant & + & 92 & + \\
\hline 349 & 50 & M & Colon & Signet ring cell carcinoma & T3NOMO & - & II & Malignant & + & 87 & + \\
\hline 350 & 57 & $\mathrm{~F}$ & Colon & Adenocarcinoma & T3NOMO & 2 & II & malignant & + & 89 & + \\
\hline 351 & 57 & $\mathrm{~F}$ & Colon & Adenocarcinoma & T3NOMO & 2 & II & malignant & + & 93 & + \\
\hline 352 & 61 & M & Colon & Adenocarcinoma & T4NOMO & 1 & II & malignant & + & 97 & + \\
\hline 353 & 61 & M & Colon & Adenocarcinoma & T4NOMO & 1 & II & malignant & + & 90 & + \\
\hline 354 & 27 & M & Colon & Adenocarcinoma (chronic inflammatic & T4NOMO & - & II & malignant & + & 83 & + \\
\hline 355 & 27 & M & Colon & Adenocarcinoma & T4NOMO & 2 & II & malignant & + & 105 & + \\
\hline 356 & 65 & M & Colon & Adenocarcinoma & T3N0MO & 1 & II & malignant & + & 111 & + \\
\hline 357 & 65 & M & Colon & Adenocarcinoma & T3NOMO & 1 & II & malignant & + & 124 & + \\
\hline 358 & 46 & $\mathrm{~F}$ & Colon & Adenocarcinoma & T4NOMO & 2 & ॥ & malignant & + & 127 & + \\
\hline 359 & 46 & $\mathrm{~F}$ & Colon & Adenocarcinoma & T4NOMO & 2 & II & malignant & + & 102 & + \\
\hline 360 & 32 & $\mathrm{~F}$ & Colon & Adenocarcinoma & T3NOMO & 1 & II & malignant & + & 107 & + \\
\hline 361 & 32 & $\mathrm{~F}$ & Colon & Adenocarcinoma & T3NOMO & 1 & II & malignant & + & 98 & + \\
\hline
\end{tabular}




\begin{tabular}{|c|c|c|c|c|c|c|c|c|c|c|c|}
\hline 362 & 58 & M & Colon & Adenocarcinoma & T4NOMO & 2 & II & malignant & + & 96 & + \\
\hline 363 & 58 & $M$ & Colon & Adenocarcinoma & T4NOMO & 2 & ॥ & malignant & + & 89 & + \\
\hline 364 & 54 & $M$ & Colon & Adenocarcinoma & T3NOMO & 2 & II & malignant & + & 80 & + \\
\hline 365 & 54 & $M$ & Colon & Adenocarcinoma & T3NOMO & 2 & ॥ & malignant & + & 99 & + \\
\hline 366 & 46 & $\mathrm{~F}$ & Colon & Adenocarcinoma & T3NOMO & 1 & II & malignant & + & 103 & + \\
\hline 367 & 46 & $\mathrm{~F}$ & Colon & Adenocarcinoma & T3NOMO & 1 & $\|$ & malignant & + & 108 & + \\
\hline 368 & 39 & $M$ & Colon & Adenocarcinoma & T4NOMO & 2 & ॥ & malignant & + & 113 & + \\
\hline 369 & 39 & M & Colon & Adenocarcinoma & T4NOMO & 2 & II & malignant & + & 121 & + \\
\hline 370 & 43 & $M$ & Colon & Adenocarcinoma & T3NOMO & 1 & ॥ & malignant & + & 127 & + \\
\hline 371 & 43 & M & Colon & Adenocarcinoma & T3N0M0 & 1 & II & malignant & + & 108 & + \\
\hline 372 & 57 & M & Colon & Adenocarcinoma & T3NOMO & 1 & ॥ & malignant & + & 103 & + \\
\hline 373 & 57 & M & Colon & Adenocarcinoma & T3NOMO & 1 & ॥ & malignant & + & 99 & + \\
\hline 374 & 59 & $\mathrm{~F}$ & Colon & Adenocarcinoma & T4NOMO & 1 & II & malignant & + & 84 & ++ \\
\hline 375 & 59 & $\mathrm{~F}$ & Colon & Adenocarcinoma & T4NOMO & 1 & ॥ & malignant & + & 81 & ++ \\
\hline 376 & 44 & $\mathrm{~F}$ & Colon & Adenocarcinoma (sparse) with necro & T3NOMO & 2 & II & malignant & + & 93 & + \\
\hline 377 & 44 & $\mathrm{~F}$ & Colon & Adenocarcinoma (necrotic tissue) & T3NOMO & - & II & malignant & + & 102 & + \\
\hline 378 & 57 & $\mathrm{~F}$ & Colon & Adenocarcinoma & T3NOMO & 2 & II & malignant & + & 109 & + \\
\hline 379 & 57 & $\mathrm{~F}$ & Colon & Adenocarcinoma & T3NOMO & 2 & ॥ & malignant & + & 114 & + \\
\hline 380 & 60 & M & Colon & Adenocarcinoma & T3NOMO & 2 & ॥ & malignant & + & 106 & + \\
\hline 381 & 60 & $M$ & Colon & Adenocarcinoma & T3NOMO & 2 & II & malignant & + & 109 & + \\
\hline 382 & 50 & $\mathrm{~F}$ & Colon & Adenocarcinoma & T3NOMO & 2 & ॥ & malignant & + & 112 & + \\
\hline 383 & 50 & $\mathrm{~F}$ & Colon & Adenocarcinoma & T3NOMO & 2 & ॥ & malignant & + & 126 & + \\
\hline 384 & 82 & $\mathrm{~F}$ & Colon & Adenocarcinoma & T3NOMO & 2 & II & malignant & + & 132 & + \\
\hline 385 & 82 & $\mathrm{~F}$ & Colon & Adenocarcinoma & T3NOMO & 2 & ॥ & malignant & + & 134 & + \\
\hline 386 & 37 & $M$ & Colon & Adenocarcinoma & T3NOMO & 2 & II & malignant & + & 138 & + \\
\hline 387 & 37 & $M$ & Colon & Adenocarcinoma & T3NOMO & 2 & II & malignant & + & 106 & + \\
\hline 388 & 72 & $\mathrm{~F}$ & Colon & Adenocarcinoma & T4NOMO & 2 & ॥ & malignant & + & 97 & + \\
\hline 389 & 72 & $\mathrm{~F}$ & Colon & Adenocarcinoma & T4NOMO & 2 & II & malignant & + & 82 & + \\
\hline 390 & 29 & $\mathrm{~F}$ & Colon & Adenocarcinoma & T3NOMO & 2 & II & malignant & + & 84 & + \\
\hline 391 & 29 & $\mathrm{~F}$ & Colon & Adenocarcinoma & T3NOMO & 2 & II & malignant & + & 90 & + \\
\hline 392 & 50 & $M$ & Colon & Adenocarcinoma & T3NOMO & 2 & II & malignant & + & 93 & + \\
\hline 393 & 50 & M & Colon & Adenocarcinoma & T3NOMO & 2 & II & malignant & + & 104 & + \\
\hline 394 & 26 & M & Colon & Adenocarcinoma & T3NOMO & 2 & II & malignant & + & 108 & + \\
\hline 395 & 26 & M & Colon & Adenocarcinoma & T3NOMO & 2 & II & malignant & + & 117 & + \\
\hline 396 & 82 & $\mathrm{~F}$ & Colon & Adenocarcinoma & T3NOMO & 2 & II & malignant & + & 89 & + \\
\hline 397 & 82 & $\mathrm{~F}$ & Colon & Adenocarcinoma & T3NOMO & 2 & II & malignant & + & 92 & + \\
\hline
\end{tabular}




\begin{tabular}{|c|c|c|c|c|c|c|c|c|c|c|c|c|}
\hline 398 & 63 & $\mathrm{~F}$ & Colon & Adenocarcinoma & T3NOMO & 2 & II & malignant & + & 95 & + & 4 \\
\hline 399 & 63 & $\mathrm{~F}$ & Colon & Adenocarcinoma & T3NOMO & 2 & II & malignant & + & 86 & + & 4 \\
\hline 400 & 73 & $M$ & Colon & Adenocarcinoma & T3NOMO & 2 & II & malignant & + & 84 & + & 4 \\
\hline 401 & 73 & $M$ & Colon & Adenocarcinoma & T3N0M0 & 2 & II & malignant & + & 82 & + & 4 \\
\hline 402 & 70 & $\mathrm{~F}$ & Colon & Adenocarcinoma & T3NOMO & 2 & II & malignant & + & 81 & + & 4 \\
\hline 403 & 70 & $\mathrm{~F}$ & Colon & Adenocarcinoma & T3NOMO & 2 & II & malignant & + & 83 & + & 4 \\
\hline 404 & 61 & $\mathrm{~F}$ & Colon & Adenocarcinoma & T3NOMO & 2 & II & malignant & + & 96 & + & 4 \\
\hline 405 & 61 & $\mathrm{~F}$ & Colon & Adenocarcinoma & T3NOMO & 2 & II & malignant & + & 92 & + & 4 \\
\hline 406 & 41 & $\mathrm{~F}$ & Colon & Adenocarcinoma & T3NOMO & 2 & II & malignant & + & 103 & + & 4 \\
\hline 407 & 41 & $\mathrm{~F}$ & Colon & Adenocarcinoma & T3N0M0 & 2 & II & malignant & + & 118 & + & 4 \\
\hline 408 & 44 & M & Colon & Adenocarcinoma & T4N0M0 & 2 & II & malignant & + & 99 & + & 4 \\
\hline 409 & 44 & M & Colon & Adenocarcinoma & T4NOMO & 2 & ॥ & malignant & + & 80 & + & 4 \\
\hline 410 & 33 & $\mathrm{~F}$ & Colon & Adenocarcinoma & T4N0M0 & 2 & II & malignant & + & 76 & +++ & 4 \\
\hline 411 & 33 & $\mathrm{~F}$ & Colon & Adenocarcinoma & T4NOMO & 2 & II & malignant & + & 83 & + & 4 \\
\hline 412 & 75 & M & Colon & Adenocarcinoma & T4NOMO & 2 & II & malignant & + & 102 & + & 4 \\
\hline 413 & 75 & M & Colon & Adenocarcinoma & T4N0M0 & 2 & II & malignant & + & 118 & + & 4 \\
\hline 414 & 50 & M & Colon & Adenocarcinoma & T4NOMO & 3 & II & malignant & + & 97 & + & 4 \\
\hline 415 & 50 & M & Colon & Adenocarcinoma & T4NOMO & 3 & II & malignant & + & 92 & + & 4 \\
\hline 416 & 75 & M & Colon & Adenocarcinoma & T4NOMO & 2 & II & malignant & + & 89 & + & 4 \\
\hline 417 & 75 & $M$ & Colon & Adenocarcinoma & T4N0M0 & 2 & II & malignant & + & 83 & + & 4 \\
\hline 418 & 44 & M & Colon & Adenocarcinoma & T4N0M0 & 3 & II & malignant & + & 138 & + & 4 \\
\hline 419 & 44 & M & Colon & Adenocarcinoma & T4NOMO & 3 & II & malignant & + & 125 & + & 4 \\
\hline 420 & 52 & $\mathrm{~F}$ & Colon & Adenocarcinoma (sparse) & T3NOMO & - & II & malignant & + & 128 & + & 4 \\
\hline 421 & 52 & $\mathrm{~F}$ & Colon & Adenocarcinoma & T3NOMO & 2 & II & malignant & + & 87 & + & 4 \\
\hline 422 & 57 & $\mathrm{~F}$ & Colon & Mucinous adenocarcinoma & T3N0M0 & 1 & II & malignant & + & 88 & + & 4 \\
\hline 423 & 57 & $\mathrm{~F}$ & Colon & Mucinous adenocarcinoma & T3NOMO & 1 & II & malignant & + & 90 & + & 4 \\
\hline 424 & 51 & $\mathrm{~F}$ & Colon & Mucinous adenocarcinoma (sparse) & T4NOMO & 2 & II & malignant & + & 93 & + & 4 \\
\hline 425 & 51 & $\mathrm{~F}$ & Colon & Mucinous adenocarcinoma & T4NOMO & 2 & II & malignant & + & 107 & + & 4 \\
\hline 426 & 56 & M & Colon & Squamous cell carcinoma & T3NOMO & 1 & II & malignant & + & 119 & + & 4 \\
\hline 427 & 56 & M & Colon & Squamous cell carcinoma & T3NOMO & 1 & II & malignant & + & 120 & + & 4 \\
\hline 428 & 62 & $\mathrm{~F}$ & Colon & Adenocarcinoma & T4N0M0 & 1 & II & malignant & + & 98 & + & 4 \\
\hline 429 & 62 & $\mathrm{~F}$ & Colon & Adenocarcinoma & T4N0M0 & 1 & II & malignant & + & 95 & + & 4 \\
\hline 430 & 41 & $\mathrm{~F}$ & Colon & Adenocarcinoma & T3NOMO & 1 & II & malignant & + & 85 & + & 4 \\
\hline 431 & 41 & $\mathrm{~F}$ & Colon & Adenocarcinoma & T3NOMO & 1 & II & malignant & + & 83 & + & 4 \\
\hline 432 & 68 & $\mathrm{~F}$ & Colon & Adenocarcinoma & T3NOMO & 2 & II & malignant & + & 91 & + & 4 \\
\hline 433 & 68 & $\mathrm{~F}$ & Colon & Adenocarcinoma & T3NOMO & 2 & II & malignant & + & 82 & + & 4 \\
\hline
\end{tabular}




\begin{tabular}{|c|c|c|c|c|c|c|c|c|c|c|c|}
\hline 434 & 34 & $\mathrm{M}$ & Colon & Adenocarcinoma & T3NOMO & 1 & II & malignant & + & 73 & + \\
\hline 435 & 34 & M & Colon & Adenocarcinoma & T3NOMO & 1 & II & malignant & + & 78 & + \\
\hline 436 & 72 & $\mathrm{~F}$ & Colon & Adenocarcinoma & T3NOMO & 1 & II & malignant & + & 76 & + \\
\hline 437 & 72 & $\mathrm{~F}$ & Colon & Adenocarcinoma & T3NOMO & 1 & II & malignant & + & 82 & + \\
\hline 438 & 51 & M & Colon & Adenocarcinoma & T3NOMO & 2 & II & malignant & + & 93 & + \\
\hline 439 & 51 & M & Colon & Adenocarcinoma & T3NOMO & 2 & II & malignant & + & 97 & + \\
\hline 440 & 52 & M & Colon & Adenocarcinoma (smooth muscle) & T4N0MO & - & II & malignant & + & 102 & + \\
\hline 441 & 52 & M & Colon & Adenocarcinoma & T4NOMO & 2 & II & malignant & + & 116 & + \\
\hline 442 & 72 & M & Colon & Adenocarcinoma & T3N0M0 & 2 & II & malignant & + & 113 & + \\
\hline 443 & 72 & M & Colon & Adenocarcinoma & T3NOMO & 2 & II & malignant & + & 128 & + \\
\hline 444 & 73 & M & Colon & Adenocarcinoma & T3N0M0 & 2 & II & malignant & + & 124 & + \\
\hline 445 & 73 & $M$ & Colon & Adenocarcinoma & T3NOMO & 2 & II & malignant & + & 108 & + \\
\hline 446 & 42 & $\mathrm{~F}$ & Colon & Adenocarcinoma & T3NOMO & 2 & II & malignant & + & 89 & + \\
\hline 447 & 42 & $\mathrm{~F}$ & Colon & Adenocarcinoma & T3NOMO & 2 & II & malignant & + & 92 & + \\
\hline 448 & 45 & M & Colon & Adenocarcinoma & T3NOMO & 2 & II & malignant & + & 94 & + \\
\hline 449 & 45 & $M$ & Colon & Adenocarcinoma & T3NOMO & 2 & II & malignant & + & 90 & + \\
\hline 450 & 43 & M & Colon & Adenocarcinoma & T3NOMO & 2 & II & malignant & + & 97 & + \\
\hline 451 & 43 & $M$ & Colon & Adenocarcinoma & T3NOMO & 2 & II & malignant & + & 88 & + \\
\hline 452 & 32 & M & Colon & Adenocarcinoma (necrotic tissue) & T3NOMO & - & II & malignant & + & 93 & + \\
\hline 453 & 32 & M & Colon & Adenocarcinoma (necrotic tissue) & T3NOMO & - & II & malignant & + & 125 & + \\
\hline 454 & 46 & M & Colon & Adenocarcinoma & T3NOMO & 2 & II & malignant & + & 118 & + \\
\hline 455 & 46 & M & Colon & Adenocarcinoma & T3NOMO & 2 & II & malignant & + & 109 & + \\
\hline 456 & 79 & $\mathrm{~F}$ & Colon & Adenocarcinoma & T3NOMO & 2 & II & malignant & + & 102 & + \\
\hline 457 & 79 & $\mathrm{~F}$ & Colon & Adenocarcinoma & T3NOMO & 2 & II & malignant & + & 89 & + \\
\hline 458 & 67 & $\mathrm{~F}$ & Colon & Adenocarcinoma & T3NOMO & 2 & II & malignant & + & 93 & + \\
\hline 459 & 67 & $\mathrm{~F}$ & Colon & Adenocarcinoma & T3NOMO & 2 & II & malignant & + & 96 & + \\
\hline 460 & 36 & $\mathrm{~F}$ & Colon & Adenocarcinoma with necrosis & T3NOMO & 2 & II & malignant & + & 97 & + \\
\hline 461 & 36 & $\mathrm{~F}$ & Colon & Adenocarcinoma (fibrous tissue and & I T3NOMO & - & II & malignant & + & 102 & + \\
\hline 462 & 32 & $\mathrm{~F}$ & Colon & Adenocarcinoma & T3NOMO & 2 & II & malignant & + & 117 & + \\
\hline 463 & 32 & $\mathrm{~F}$ & Colon & Adenocarcinoma & T3NOMO & 2 & II & malignant & + & 103 & + \\
\hline 464 & 62 & $\mathrm{~F}$ & Colon & Adenocarcinoma & T3NOMO & 2 & II & malignant & + & 98 & + \\
\hline 465 & 62 & $\mathrm{~F}$ & Colon & Adenocarcinoma & T3NOMO & 2 & II & malignant & + & 95 & + \\
\hline 466 & 67 & $\mathrm{~F}$ & Colon & Adenocarcinoma & T3NOMO & 2 & II & malignant & + & 90 & + \\
\hline 467 & 67 & $\mathrm{~F}$ & Colon & Adenocarcinoma & T3NOMO & 2 & II & malignant & + & 83 & + \\
\hline 468 & 38 & M & Colon & Adenocarcinoma & T4NOMO & 2 & II & malignant & + & 87 & + \\
\hline 469 & 38 & $M$ & Colon & Adenocarcinoma & T4NOMO & 2 & II & malignant & + & 82 & + \\
\hline
\end{tabular}




\begin{tabular}{|c|c|c|c|c|c|c|c|c|c|c|c|c|}
\hline 470 & 48 & $\mathrm{~F}$ & Colon & Adenocarcinoma with necrosis & T4N0M0 & - & II & malignant & + & 93 & + & 4 \\
\hline 471 & 48 & $\mathrm{~F}$ & Colon & Adenocarcinoma with necrosis & T4N0M0 & - & II & malignant & + & 98 & + & 4 \\
\hline 472 & 43 & M & Colon & Adenocarcinoma & T3NOMO & 2 & II & malignant & + & 87 & + & 4 \\
\hline 473 & 43 & M & Colon & Adenocarcinoma & T3NOMO & 2 & ॥ & malignant & + & 105 & + & 4 \\
\hline 474 & 63 & $\mathrm{~F}$ & Colon & Adenocarcinoma & T4N0M0 & 2 & II & malignant & + & 127 & + & 4 \\
\hline 475 & 63 & $\mathrm{~F}$ & Colon & Adenocarcinoma (sparse) & T4N0M0 & 2 & II & malignant & + & 119 & + & 4 \\
\hline 476 & 58 & $\mathrm{~F}$ & Colon & Adenocarcinoma with necrosis & T3N0M0 & 2 & II & malignant & + & 134 & + & 4 \\
\hline 477 & 58 & $\mathrm{~F}$ & Colon & Adenocarcinoma (necrotic tissue) & T3N0M0 & - & II & malignant & + & 135 & + & 4 \\
\hline 478 & 62 & $\mathrm{~F}$ & Colon & Adenocarcinoma & T4N0M0 & 2 & II & malignant & + & 128 & + & 4 \\
\hline 479 & 62 & $\mathrm{~F}$ & Colon & Adenocarcinoma & T4N0M0 & 2 & II & malignant & + & 124 & + & 4 \\
\hline 480 & 57 & $\mathrm{~F}$ & Colon & Adenocarcinoma & T3NOMO & 2 & II & malignant & + & 103 & + & 4 \\
\hline 481 & 57 & $\mathrm{~F}$ & Colon & Adenocarcinoma & T3NOMO & 2 & II & malignant & + & 116 & + & 4 \\
\hline 482 & 47 & M & Colon & Adenocarcinoma & T3NOMO & 2 & II & malignant & + & 98 & + & 4 \\
\hline 483 & 47 & M & Colon & Adenocarcinoma & T3N0M0 & 2 & II & malignant & + & 92 & + & 4 \\
\hline 484 & 60 & M & Colon & Adenocarcinoma & T3N0M0 & 2 & II & malignant & + & 86 & + & 4 \\
\hline 485 & 60 & M & Colon & Adenocarcinoma & T3NOMO & 2 & II & malignant & + & 93 & + & 4 \\
\hline 486 & 64 & M & Colon & Adenocarcinoma & T4NOMO & 2 & II & malignant & + & 85 & + & 4 \\
\hline 487 & 64 & M & Colon & Adenocarcinoma & T4NOMO & 2 & II & malignant & + & 92 & + & 4 \\
\hline 488 & 32 & M & Colon & Adenocarcinoma & T3NOMO & 2 & II & malignant & + & 98 & + & 4 \\
\hline 489 & 32 & $M$ & Colon & Adenocarcinoma & T3N0M0 & 2 & II & malignant & + & 84 & + & 4 \\
\hline 490 & 46 & $\mathrm{~F}$ & Colon & Adenocarcinoma & T3N0M0 & 2 & II & malignant & + & 87 & + & 4 \\
\hline 491 & 46 & $\mathrm{~F}$ & Colon & Adenocarcinoma & T3NOMO & 2 & II & malignant & + & 103 & + & 4 \\
\hline 492 & 57 & M & Colon & Adenocarcinoma & T3NOMO & 2 & II & malignant & + & 119 & + & 4 \\
\hline 493 & 57 & M & Colon & Adenocarcinoma & T3NOMO & 2 & II & malignant & + & 124 & + & 4 \\
\hline 494 & 67 & $\mathrm{~F}$ & Colon & Adenocarcinoma & T3NOMO & 2 & II & malignant & + & 107 & + & 4 \\
\hline 495 & 67 & $\mathrm{~F}$ & Colon & Adenocarcinoma & T3NOMO & 2 & II & malignant & + & 104 & + & 4 \\
\hline 496 & 55 & $\mathrm{~F}$ & Colon & Adenocarcinoma & T3N0MO & 2 & II & malignant & + & 89 & + & 4 \\
\hline 497 & 55 & $\mathrm{~F}$ & Colon & Adenocarcinoma (sparse) with necro: & T3NOMO & - & II & malignant & + & 82 & + & 4 \\
\hline 498 & 69 & $\mathrm{~F}$ & Colon & Adenocarcinoma & T3NOMO & 2 & II & malignant & + & 84 & + & 4 \\
\hline 499 & 69 & $\mathrm{~F}$ & Colon & Adenocarcinoma (chronic inflammati & ( T3NOMO & - & II & malignant & + & 87 & + & 4 \\
\hline 500 & 74 & M & Colon & Adenocarcinoma & T3N0MO & 2 & II & malignant & + & 90 & + & 4 \\
\hline 501 & 74 & M & Colon & Adenocarcinoma (fibrofatty tissue) & T3NOMO & - & II & malignant & + & 99 & + & 4 \\
\hline 502 & 53 & M & Colon & Adenocarcinoma & T3N0M0 & 2 & II & malignant & + & 102 & + & 4 \\
\hline 503 & 53 & M & Colon & Adenocarcinoma & T3N0M0 & 2 & II & malignant & + & 106 & + & 4 \\
\hline 504 & 40 & M & Colon & Adenocarcinoma & T3N0M0 & 2 & II & malignant & + & 113 & + & 4 \\
\hline 505 & 40 & M & Colon & Adenocarcinoma & T3NOMO & 2 & II & malignant & + & 94 & + & 4 \\
\hline
\end{tabular}




\begin{tabular}{|c|c|c|c|c|c|c|c|c|c|c|c|}
\hline 506 & 73 & $\mathrm{M}$ & Colon & Adenocarcinoma & T3NOMO & 2 & II & malignant & + & 86 & + \\
\hline 507 & 73 & $M$ & Colon & Adenocarcinoma & T3NOMO & 2 & II & malignant & + & 88 & + \\
\hline 508 & 65 & $M$ & Colon & Adenocarcinoma & T3NOMO & 2 & II & malignant & + & 90 & + \\
\hline 509 & 65 & $M$ & Colon & Adenocarcinoma & T3N0M0 & 2 & II & malignant & + & 91 & + \\
\hline 510 & 56 & $\mathrm{~F}$ & Colon & Adenocarcinoma & T4N0M0 & $2--3$ & II & malignant & + & 97 & + \\
\hline 511 & 56 & $\mathrm{~F}$ & Colon & Adenocarcinoma & T4NOMO & $2--2$ & II & malignant & + & 81 & + \\
\hline 512 & 78 & M & Colon & Adenocarcinoma & T3NOMO & $2--3$ & II & malignant & + & 110 & + \\
\hline 513 & 78 & M & Colon & Adenocarcinoma & T3NOMO & $2--3$ & ॥ & malignant & + & 124 & + \\
\hline 514 & 74 & $\mathrm{~F}$ & Colon & Mucinous adenocarcinoma & T3N0M0 & 2 & II & malignant & + & 128 & + \\
\hline 515 & 74 & $\mathrm{~F}$ & Colon & Mucinous adenocarcinoma & T3NOMO & 2 & II & malignant & + & 135 & + \\
\hline 516 & 47 & M & Colon & Adenocarcinoma & T3N0M0 & 2 & II & malignant & + & 84 & + \\
\hline 517 & 47 & M & Colon & Adenocarcinoma & T3NOMO & 2 & II & malignant & + & 83 & + \\
\hline 518 & 50 & $\mathrm{~F}$ & Colon & Adenocarcinoma & T4NOMO & 3 & II & malignant & + & 109 & + \\
\hline 519 & 50 & $\mathrm{~F}$ & Colon & Adenocarcinoma (chronic inflammati & i T4NOMO & - & II & malignant & + & 87 & + \\
\hline 520 & 39 & $\mathrm{~F}$ & Colon & Adenocarcinoma & T3NOMO & 3 & II & malignant & + & 112 & + \\
\hline 521 & 39 & $\mathrm{~F}$ & Colon & Adenocarcinoma & T3NOMO & 3 & II & malignant & + & 105 & + \\
\hline 522 & 74 & M & Colon & Adenocarcinoma & T3NOMO & 3 & II & malignant & + & 89 & + \\
\hline 523 & 74 & M & Colon & Adenocarcinoma & T3NOMO & 3 & II & malignant & + & 93 & + \\
\hline 524 & 46 & M & Colon & Adenocarcinoma & T4NOM0 & 3 & II & malignant & + & 128 & + \\
\hline 525 & 46 & M & Colon & Adenocarcinoma & T4NOMO & 3 & II & malignant & + & 103 & + \\
\hline 526 & 52 & M & Colon & Adenocarcinoma & T3NOMO & 3 & II & malignant & + & 106 & + \\
\hline 527 & 52 & M & Colon & Adenocarcinoma & T3N0M0 & 3 & II & malignant & + & 97 & + \\
\hline 528 & 72 & M & Colon & Mucinous adenocarcinoma & T3NOMO & 3 & ॥ & malignant & + & 92 & + \\
\hline 529 & 72 & M & Colon & Mucinous adenocarcinoma & T3NOMO & 3 & II & malignant & + & 84 & + \\
\hline 530 & 59 & M & Colon & Mucinous adenocarcinoma & T3NOMO & 3 & II & malignant & + & 76 & + \\
\hline 531 & 59 & M & Colon & Mucinous adenocarcinoma & T3NOMO & 3 & II & malignant & + & 84 & + \\
\hline 532 & 55 & $\mathrm{~F}$ & Colon & Adenocarcinoma (mucosa of colon) & T4NOMO & - & II & malignant & + & 143 & + \\
\hline 533 & 55 & $\mathrm{~F}$ & Colon & Mucinous adenocarcinoma & T4NOMO & 3 & II & malignant & + & 135 & + \\
\hline 534 & 35 & M & Colon & Mucinous adenocarcinoma & T3NOMO & 3 & II & malignant & + & 127 & + \\
\hline 535 & 35 & M & Colon & Mucinous adenocarcinoma & T3NOMO & 3 & II & malignant & + & 138 & + \\
\hline 536 & 72 & M & Colon & Signet-ring cell carcinoma & T3NOMO & - & II & malignant & + & 106 & + \\
\hline 537 & 72 & M & Colon & Signet-ring cell carcinoma & T3NOMO & - & II & malignant & + & 117 & + \\
\hline 538 & 47 & M & Colon & Mucinous adenocarcinoma & T2 NO MO & & $\| A$ & tumor & + & 86 & + \\
\hline 539 & 44 & M & Colon & Mucinous adenocarcinoma & T3 NO MO & & IIA & tumor & + & 83 & + \\
\hline 540 & 33 & M & Colon & Partly mucinous adenocarcinoma & T3 NO MO & & IIA & tumor & + & 92 & + \\
\hline 541 & 70 & M & Colon & Partly mucinous adenocarcinoma & T3 NO MO & & IIA & tumor & + & 84 & + \\
\hline
\end{tabular}




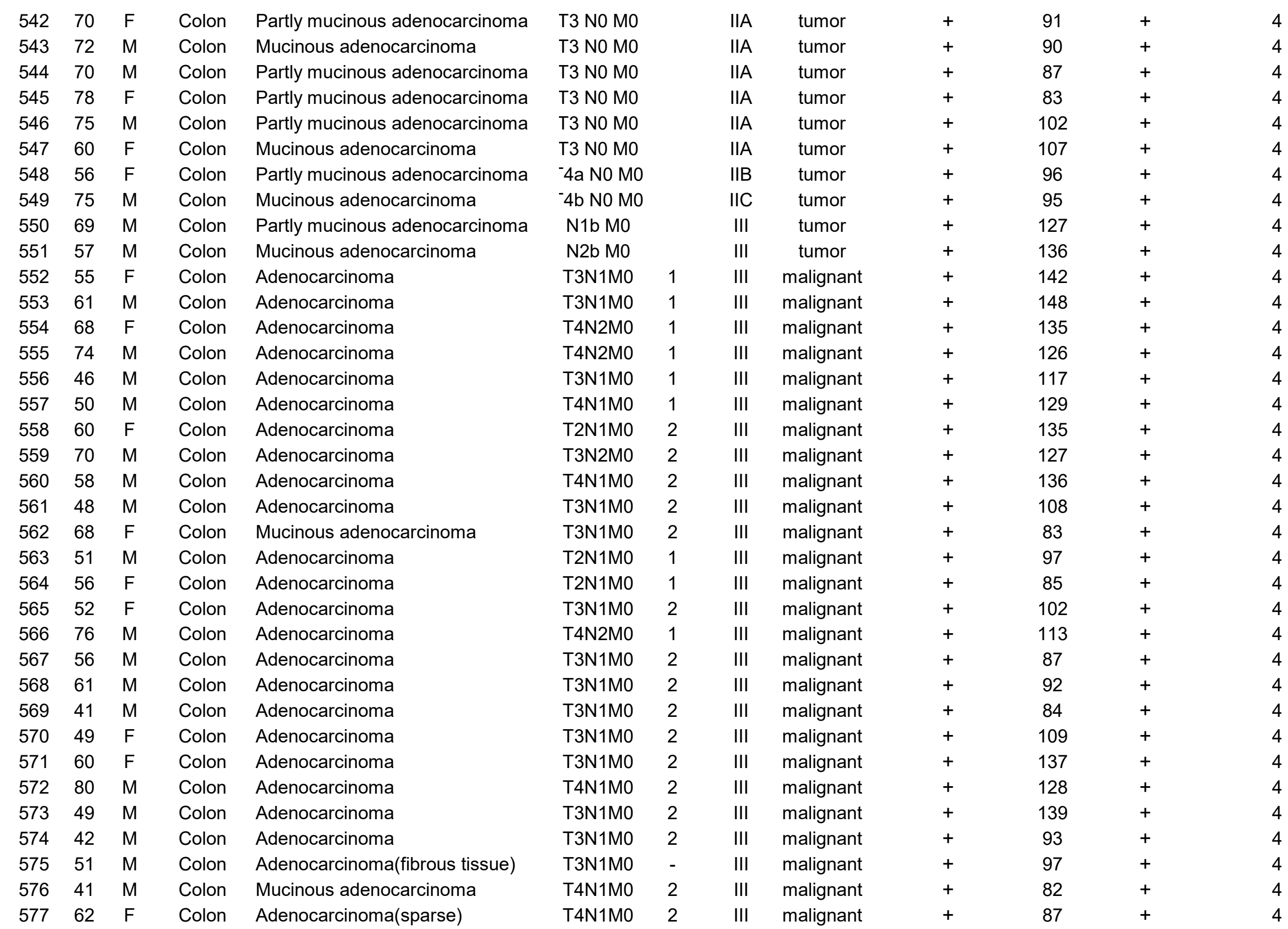




\begin{tabular}{|c|c|c|c|c|}
\hline 578 & 38 & $M$ & Colon & Adenocarcinoma \\
\hline 579 & 65 & $M$ & Colon & Adenocarcinoma \\
\hline 580 & 58 & $\mathrm{~F}$ & Colon & Adenocarcinoma \\
\hline 581 & 41 & $\mathrm{~F}$ & Colon & Adenocarcinoma \\
\hline 582 & 48 & $\mathrm{~F}$ & Colon & Adenocarcinoma \\
\hline 583 & 78 & $\mathrm{~F}$ & Colon & Adenocarcinoma \\
\hline 584 & 72 & $\mathrm{~F}$ & Colon & Adenocarcinoma \\
\hline 585 & 47 & $\mathrm{~F}$ & Colon & Adenocarcinoma \\
\hline 586 & 64 & M & Colon & Adenocarcinoma \\
\hline 587 & 54 & $\mathrm{~F}$ & Colon & Adenocarcinoma \\
\hline 588 & 52 & $\mathrm{~F}$ & Colon & Adenocarcinoma \\
\hline 589 & 45 & M & Colon & Adenocarcinoma \\
\hline 590 & 70 & $\mathrm{~F}$ & Colon & Adenocarcinoma(necrosis) \\
\hline 591 & 69 & $\mathrm{~F}$ & Colon & Adenocarcinoma \\
\hline 592 & 61 & M & Colon & Adenocarcinoma \\
\hline 593 & 74 & $M$ & Colon & Adenocarcinoma \\
\hline 594 & 46 & M & Colon & Mucinous adenocarcinoma \\
\hline 595 & 53 & $\mathrm{~F}$ & Colon & Mucinous adenocarcinoma \\
\hline 596 & 37 & $\mathrm{~F}$ & Colon & Mucinous adenocarcinoma \\
\hline 597 & 57 & M & Colon & Mucinous adenocarcinoma \\
\hline 598 & 45 & $\mathrm{~F}$ & Colon & Adenocarcinoma \\
\hline 599 & 62 & $\mathrm{~F}$ & Colon & Adenocarcinoma \\
\hline 600 & 40 & M & Colon & Adenocarcinoma \\
\hline 601 & 73 & $\mathrm{~F}$ & Colon & Adenocarcinoma \\
\hline 602 & 20 & $\mathrm{~F}$ & Colon & Adenocarcinoma \\
\hline 603 & 38 & $\mathrm{~F}$ & Colon & Adenocarcinoma \\
\hline 604 & 34 & $M$ & Colon & Mucinous adenocarcinoma \\
\hline 605 & 56 & M & Colon & Signet ring cell carcinoma \\
\hline 606 & 56 & $\mathrm{~F}$ & Colon & Signet ring cell carcinoma \\
\hline 607 & 81 & $\mathrm{~F}$ & Colon & Signet ring cell carcinoma \\
\hline 608 & 68 & M & Colon & Squamous cell carcinoma \\
\hline 609 & 73 & $\mathrm{~F}$ & Colon & Adenocarcinoma \\
\hline 610 & 55 & M & Colon & Adenocarcinoma \\
\hline 611 & 59 & $\mathrm{~F}$ & Colon & Adenocarcinoma \\
\hline 612 & 43 & $F$ & Colon & Adenocarcinoma \\
\hline 613 & 60 & M & Colon & Adenocarcinoma \\
\hline
\end{tabular}

\begin{tabular}{|c|c|c|c|c|c|c|c|}
\hline T4N1M0 & 2 & III & malignant & + & 80 & + & 4 \\
\hline T3N1M0 & 2 & III & malignant & + & 137 & + & 4 \\
\hline T3N1M0 & 2 & III & malignant & + & 135 & + & 4 \\
\hline T3N1M0 & 2 & III & malignant & + & 128 & + & 4 \\
\hline T4N1M0 & 2 & III & malignant & + & 116 & + & 4 \\
\hline T3N1M0 & 2 & III & malignant & + & 109 & + & 4 \\
\hline T3N1M0 & 2 & III & malignant & + & 105 & + & 4 \\
\hline T3N1M0 & 2 & III & malignant & + & 142 & + & 4 \\
\hline T3N1M0 & 2 & III & malignant & + & 147 & + & 4 \\
\hline T4N1M0 & 2 & III & malignant & + & 137 & + & 4 \\
\hline T3N1M0 & 2 & III & malignant & + & 129 & + & 4 \\
\hline T4N1M0 & 1 & III & malignant & + & 108 & + & 4 \\
\hline T3N1M0 & 2 & III & malignant & + & 86 & + & 4 \\
\hline T3N1M0 & 2 & III & malignant & + & 88 & + & 4 \\
\hline T3N2M0 & 2 & III & malignant & + & 92 & + & 4 \\
\hline T3N1M0 & 2 & III & malignant & + & 90 & + & 4 \\
\hline T3N2M0 & 1 & III & malignant & + & 93 & + & 4 \\
\hline T3N1M0 & 2 & III & malignant & + & 82 & + & 4 \\
\hline T4N2M0 & 3 & III & malignant & + & 91 & + & 4 \\
\hline T3N1M0 & 2 & III & malignant & + & 99 & + & 4 \\
\hline T4N2M0 & 1 & III & malignant & + & 83 & +++ & 4 \\
\hline T3N1M0 & 3 & III & malignant & + & 89 & + & 4 \\
\hline T4N2M0 & 3 & III & malignant & + & 103 & + & 4 \\
\hline T4N1M0 & 3 & III & malignant & + & 83 & + & 4 \\
\hline T4N1M0 & 3 & III & malignant & + & 89 & + & 4 \\
\hline T4N1M0 & 3 & III & malignant & + & 91 & + & 4 \\
\hline T3N1M0 & 3 & III & malignant & + & 98 & + & 4 \\
\hline T4N2M0 & - & III & malignant & + & 106 & + & 4 \\
\hline T3N1M0 & - & III & malignant & + & 109 & + & 4 \\
\hline T4N2M0 & - & III & malignant & + & 112 & + & 4 \\
\hline T4N1M0 & 2 & III & malignant & + & 86 & + & 4 \\
\hline T3N1M0 & 1 & III & Malignant & + & 89 & + & 4 \\
\hline T3N2M0 & 1 & III & Malignant & + & 93 & + & 4 \\
\hline T3N2M0 & 1 & III & Malignant & + & 95 & + & 4 \\
\hline T4N1M0 & 2 & III & Malignant & + & 104 & + & 4 \\
\hline T4N2M0 & 1 & III & Malignant & + & 124 & + & 4 \\
\hline
\end{tabular}




\begin{tabular}{|c|c|c|c|c|c|c|c|c|c|c|c|}
\hline 614 & 65 & $M$ & Colon & Adenocarcinoma & T4N1M0 & 2 & III & Malignant & + & 128 & + \\
\hline 615 & 45 & M & Colon & Adenocarcinoma & T3N1M0 & 1 & III & Malignant & + & 109 & + \\
\hline 616 & 30 & $\mathrm{~F}$ & Colon & Adenocarcinoma & T3N2M0 & 1 & III & Malignant & + & 114 & + \\
\hline 617 & 78 & M & Colon & Adenocarcinoma & T4N1M0 & 2 & III & Malignant & + & 87 & + \\
\hline 618 & 49 & $\mathrm{~F}$ & Colon & Adenocarcinoma & T4N1M0 & 2 & III & Malignant & + & 89 & + \\
\hline 619 & 51 & $\mathrm{~F}$ & Colon & Adenocarcinoma & T4N1M0 & 1 & III & Malignant & + & 93 & + \\
\hline 620 & 55 & M & Colon & Adenocarcinoma & T3N2M0 & 1 & III & Malignant & + & 97 & + \\
\hline 621 & 39 & $\mathrm{~F}$ & Colon & Mucinous adenocarcinoma & T3N2M0 & 2 & III & Malignant & + & 81 & + \\
\hline 622 & 60 & M & Colon & Adenocarcinoma & T3N1M0 & 1 & III & Malignant & + & 127 & + \\
\hline 623 & 50 & M & Colon & Mucinous adenocarcinoma (smooth r & T3N1M0 & - & III & Malignant & + & 109 & + \\
\hline 624 & 70 & $\mathrm{~F}$ & Colon & Mucinous adenocarcinoma & T2N1M0 & 1 & III & Malignant & + & 102 & + \\
\hline 625 & 45 & $\mathrm{~F}$ & Colon & Mucinous adenocarcinoma & T3N2M0 & 1 & III & Malignant & + & 87 & + \\
\hline 626 & 51 & M & Colon & Mucinous adenocarcinoma & T3N1M0 & 1 & III & Malignant & + & 93 & + \\
\hline 627 & 40 & $\mathrm{~F}$ & Colon & Adenocarcinoma & T3N1M0 & 1 & III & Malignant & + & 94 & + \\
\hline 628 & 54 & M & Colon & Mucinous adenocarcinoma & T3N1M0 & 3 & III & Malignant & + & 98 & + \\
\hline 629 & 55 & M & Colon & Mucinous adenocarcinoma (sparse) & T3N2M0 & 1 & III & Malignant & + & 103 & + \\
\hline 630 & 56 & $\mathrm{~F}$ & Colon & Mucinous adenocarcinoma & T4N1M0 & 2 & III & Malignant & + & 108 & + \\
\hline 631 & 83 & $\mathrm{~F}$ & Colon & Adenocarcinoma & T3N2M0 & 3 & III & Malignant & + & 117 & + \\
\hline 632 & 40 & M & Colon & Adenocarcinoma & T4N1M0 & 2 & III & Malignant & + & 88 & + \\
\hline 633 & 60 & $\mathrm{~F}$ & Colon & Adenocarcinoma & T4N1M0 & 2 & III & Malignant & + & 84 & +++ \\
\hline 634 & 55 & M & Colon & Adenocarcinoma & T3N2M0 & 2 & III & Malignant & + & 92 & + \\
\hline 635 & 56 & M & Colon & Adenocarcinoma & T3N2M0 & 3 & III & Malignant & + & 90 & + \\
\hline 636 & 61 & $\mathrm{~F}$ & Colon & Adenocarcinoma & T3N1M0 & 2 & III & Malignant & + & 87 & + \\
\hline 637 & 64 & $\mathrm{~F}$ & Colon & Adenocarcinoma & T4N1M0 & 2 & III & Malignant & + & 84 & + \\
\hline 638 & 60 & $\mathrm{~F}$ & Colon & Adenocarcinoma & T3N1M0 & 2 & III & Malignant & + & 78 & + \\
\hline 639 & 73 & $\mathrm{~F}$ & Colon & Adenocarcinoma & T4N2M0 & 2 & III & Malignant & + & 94 & + \\
\hline 640 & 42 & M & Colon & Adenocarcinoma (sparse) & T3N2M0 & 2 & III & Malignant & + & 81 & + \\
\hline 641 & 43 & M & Colon & Adenocarcinoma & T3N1M0 & 2 & III & Malignant & + & 84 & + \\
\hline 642 & 50 & $\mathrm{~F}$ & Colon & Adenocarcinoma & T3N2M0 & 2 & III & Malignant & + & 87 & + \\
\hline 643 & 43 & M & Colon & Adenocarcinoma & T4N1M0 & 2 & III & Malignant & + & 93 & + \\
\hline 644 & 51 & M & Colon & Adenocarcinoma & T3N1M0 & 2 & III & Malignant & + & 98 & + \\
\hline 645 & 35 & $\mathrm{~F}$ & Colon & Adenocarcinoma & T3N1M0 & 2 & III & Malignant & + & 96 & + \\
\hline 646 & 35 & M & Colon & Adenocarcinoma & T4N1M0 & 2 & III & Malignant & + & 84 & +++ \\
\hline 647 & 52 & $\mathrm{~F}$ & Colon & Adenocarcinoma & T3N2M0 & 2 & III & Malignant & + & 88 & + \\
\hline 648 & 61 & M & Colon & Adenocarcinoma (fibrous tissue and I & T3N1M0 & - & III & Malignant & + & 91 & + \\
\hline 649 & 47 & $\mathrm{~F}$ & Colon & Adenocarcinoma & T3N1M0 & 2 & III & Malignant & + & 94 & + \\
\hline
\end{tabular}




\begin{tabular}{|c|c|c|c|c|c|c|c|c|c|c|c|c|}
\hline 650 & 64 & $\mathrm{~F}$ & Colon & Adenocarcinoma & T3N2M0 & 2 & III & Malignant & + & 98 & + & 4 \\
\hline 651 & 70 & $\mathrm{~F}$ & Colon & Adenocarcinoma & T3N2M0 & 2 & III & Malignant & + & 104 & + & 4 \\
\hline 652 & 43 & $M$ & Colon & Adenocarcinoma & T3N1M0 & 1 & III & Malignant & + & 112 & + & 4 \\
\hline 653 & 39 & $M$ & Colon & Adenocarcinoma & T4N2M0 & 2 & III & Malignant & + & 115 & + & 4 \\
\hline 654 & 68 & $\mathrm{~F}$ & Colon & Adenocarcinoma & T2N1M0 & 2 & III & Malignant & + & 109 & + & 4 \\
\hline 655 & 57 & $\mathrm{~F}$ & Colon & Adenocarcinoma & T3N2M0 & 2 & III & Malignant & + & 107 & + & 4 \\
\hline 656 & 40 & $\mathrm{~F}$ & Colon & Adenocarcinoma (fibrous tissue and I & I T2N2M0 & - & III & Malignant & + & 124 & + & 4 \\
\hline 657 & 84 & $\mathrm{~F}$ & Colon & Adenocarcinoma (degeneration tissu & T2N2M0 & - & III & Malignant & + & 126 & + & 4 \\
\hline 658 & 65 & M & Colon & Adenocarcinoma & T3N1M0 & 2 & III & Malignant & + & 108 & + & 4 \\
\hline 659 & 30 & M & Colon & Adenocarcinoma & T2N1M0 & 2 & III & Malignant & + & 105 & + & 4 \\
\hline 660 & 58 & M & Colon & Mucinous adenocarcinoma (smooth r & T3N1M0 & - & III & Malignant & + & 94 & + & 4 \\
\hline 661 & 48 & M & Colon & Adenocarcinoma & T3N1M0 & 2 & III & Malignant & + & 85 & + & 4 \\
\hline 662 & 39 & M & Colon & Mucinous adenocarcinoma & T3N1M0 & 2 & III & Malignant & + & 89 & + & 4 \\
\hline 663 & 67 & M & Colon & Mucinous adenocarcinoma & T4N2M0 & 2 & III & Malignant & + & 93 & + & 4 \\
\hline 664 & 28 & $\mathrm{~F}$ & Colon & Mucinous adenocarcinoma & $\mathrm{T} 2 \mathrm{~N} 1 \mathrm{M} 0$ & 1 & III & Malignant & + & 94 & + & 4 \\
\hline 665 & 56 & M & Colon & Mucinous adenocarcinoma & T3N2M0 & 2 & III & Malignant & + & 96 & + & 4 \\
\hline 666 & 42 & M & Colon & Adenocarcinoma & T3N1M0 & 3 & III & Malignant & + & 103 & + & 4 \\
\hline 667 & 49 & $\mathrm{~F}$ & Colon & Adenocarcinoma & T3N2M0 & 1 & III & Malignant & + & 105 & + & 4 \\
\hline 668 & 41 & $M$ & Colon & Adenocarcinoma & T3N2M0 & 2 & III & Malignant & + & 109 & + & 4 \\
\hline 669 & 70 & M & Colon & Adenocarcinoma & T3N1M0 & 3 & III & Malignant & + & 115 & + & 4 \\
\hline 670 & 45 & M & Colon & Mucinous adenocarcinoma & T4N2M0 & 2 & III & Malignant & + & 127 & + & 4 \\
\hline 671 & 51 & M & Colon & Adenocarcinoma & T4N1M0 & 3 & III & Malignant & + & 147 & + & 4 \\
\hline 672 & 57 & $\mathrm{~F}$ & Colon & Adenocarcinoma (degeneration tissu & T3N1M0 & - & III & Malignant & + & 152 & + & 4 \\
\hline 673 & 65 & M & Colon & Adenocarcinoma & T3N1M0 & 3 & III & Malignant & + & 137 & + & 4 \\
\hline 674 & 28 & $\mathrm{~F}$ & Colon & Mucinous adenocarcinoma & T4N1M0 & 3 & III & Malignant & + & 128 & + & 4 \\
\hline 675 & 33 & $\mathrm{~F}$ & Colon & Adenocarcinoma (smooth muscle) & T4N1M0 & - & III & Malignant & + & 84 & + & 4 \\
\hline 676 & 51 & M & Colon & Adenocarcinoma & T3N2M0 & 3 & III & Malignant & + & 97 & + & 4 \\
\hline 677 & 52 & $\mathrm{~F}$ & Colon & Adenocarcinoma & T3N1M0 & 3 & III & Malignant & + & 81 & + & 4 \\
\hline 678 & 43 & $\mathrm{~F}$ & Colon & Adenocarcinoma & T3N1M0 & 3 & III & Malignant & + & 90 & + & 4 \\
\hline 679 & 55 & M & Colon & Adenocarcinoma & T4N1M0 & 3 & III & Malignant & + & 94 & + & 4 \\
\hline 680 & 59 & $\mathrm{~F}$ & Colon & Mucinous adenocarcinoma & T3N1M0 & 3 & III & Malignant & + & 97 & + & 4 \\
\hline 681 & 43 & M & Colon & Mucinous adenocarcinoma & T4N1M0 & 3 & III & Malignant & + & 99 & + & 4 \\
\hline 682 & 38 & $\mathrm{~F}$ & Colon & Signet ring cell carcinoma & T3N1M0 & - & III & Malignant & + & 120 & + & 4 \\
\hline 683 & 22 & M & Colon & Mucinous adenocarcinoma & T4N1M0 & 3 & III & Malignant & + & 118 & + & 4 \\
\hline 684 & 56 & M & Colon & Mucinous adenocarcinoma & T4N1M0 & 3 & III & Malignant & + & 84 & + & 4 \\
\hline 685 & 56 & $M$ & Colon & Mucinous adenocarcinoma & T3N1M0 & 3 & III & Malignant & + & 86 & + & 4 \\
\hline
\end{tabular}




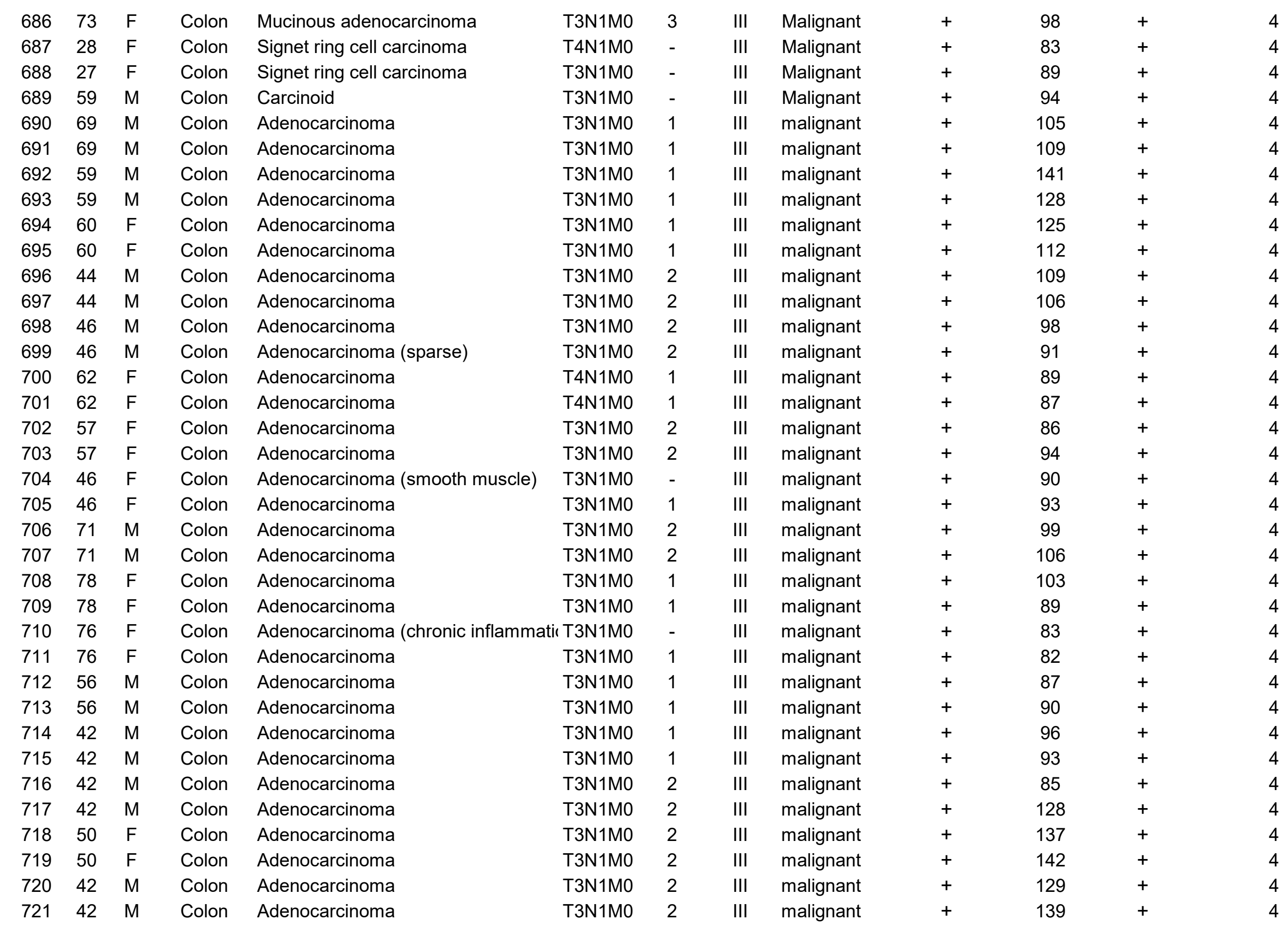




\begin{tabular}{|c|c|c|c|c|c|c|c|c|c|c|c|}
\hline 722 & 53 & $\mathrm{M}$ & Colon & Adenocarcinoma & T3N1M0 & 2 & III & malignant & + & 137 & + \\
\hline 723 & 53 & $M$ & Colon & Adenocarcinoma & T3N1M0 & 2 & III & malignant & + & 126 & + \\
\hline 724 & 56 & $M$ & Colon & Adenocarcinoma & T3N1M0 & 2 & III & malignant & + & 118 & + \\
\hline 725 & 56 & $M$ & Colon & Adenocarcinoma & T3N1M0 & 2 & III & malignant & + & 109 & + \\
\hline 726 & 63 & $\mathrm{~F}$ & Colon & Adenocarcinoma & T3N1M0 & 2 & III & malignant & + & 101 & + \\
\hline 727 & 63 & $\mathrm{~F}$ & Colon & Adenocarcinoma & T3N1M0 & 2 & III & malignant & + & 91 & + \\
\hline 728 & 56 & $\mathrm{~F}$ & Colon & Adenocarcinoma & T3N1M0 & 2 & III & malignant & + & 97 & + \\
\hline 729 & 56 & $\mathrm{~F}$ & Colon & Adenocarcinoma & T3N1M0 & 2 & III & malignant & + & 98 & + \\
\hline 730 & 60 & $M$ & Colon & Adenocarcinoma & T3N1M0 & 2 & III & malignant & + & 96 & + \\
\hline 731 & 60 & M & Colon & Adenocarcinoma & T3N1M0 & 2 & III & malignant & + & 86 & + \\
\hline 732 & 41 & $\mathrm{~F}$ & Colon & Adenocarcinoma & T3N1M0 & 2 & III & malignant & + & 85 & + \\
\hline 733 & 41 & $\mathrm{~F}$ & Colon & Adenocarcinoma & T3N1M0 & 2 & III & malignant & + & 84 & + \\
\hline 734 & 33 & M & Colon & Adenocarcinoma (sparse) & T3N1M0 & - & III & malignant & + & 93 & + \\
\hline 735 & 33 & M & Colon & Adenocarcinoma & T3N1M0 & 2 & III & malignant & + & 109 & + \\
\hline 736 & 58 & $\mathrm{~F}$ & Colon & Adenocarcinoma & T3N1M0 & 2 & III & malignant & + & 118 & + \\
\hline 737 & 58 & $\mathrm{~F}$ & Colon & Adenocarcinoma (sparse) & T3N1M0 & 2 & III & malignant & + & 87 & + \\
\hline 738 & 52 & $\mathrm{~F}$ & Colon & Adenocarcinoma & T3N1M0 & 3 & III & malignant & + & 96 & + \\
\hline 739 & 52 & $\mathrm{~F}$ & Colon & Adenocarcinoma & T3N1M0 & 3 & III & malignant & + & 82 & + \\
\hline 740 & 51 & M & Colon & Adenosquamous carcinoma & T3N1M0 & - & III & malignant & + & 84 & + \\
\hline 741 & 51 & M & Colon & Adenosquamous carcinoma & T3N1M0 & - & III & malignant & + & 83 & + \\
\hline 742 & 80 & M & Colon & Adenocarcinoma & T4N2M0 & 2 & III & malignant & + & 97 & + \\
\hline 743 & 80 & M & Colon & Adenocarcinoma & T4N2M0 & 2 & III & malignant & + & 99 & + \\
\hline 744 & 60 & M & Colon & Adenocarcinoma & T3N1M0 & 2 & III & malignant & + & 90 & + \\
\hline 745 & 60 & M & Colon & Adenocarcinoma & T3N1M0 & 2 & III & malignant & + & 93 & + \\
\hline 746 & 75 & M & Colon & Adenocarcinoma & T3N1M0 & 2 & III & malignant & + & 87 & + \\
\hline 747 & 75 & M & Colon & Adenocarcinoma & T3N1M0 & 2 & III & malignant & + & 167 & + \\
\hline 748 & 68 & $\mathrm{~F}$ & Colon & Adenocarcinoma (chronic inflammatic & (T3N1M0 & - & III & malignant & + & 156 & + \\
\hline 749 & 68 & $\mathrm{~F}$ & Colon & Adenocarcinoma (smooth muscle) & T3N1M0 & - & III & malignant & + & 152 & + \\
\hline 750 & 50 & $\mathrm{~F}$ & Colon & Adenocarcinoma & T3N1M0 & 2 & III & malignant & + & 148 & + \\
\hline 751 & 50 & $\mathrm{~F}$ & Colon & Adenocarcinoma & T3N1M0 & 2 & III & malignant & + & 135 & + \\
\hline 752 & 69 & $\mathrm{~F}$ & Colon & Adenocarcinoma & T3N1M0 & 2 & III & malignant & + & 162 & + \\
\hline 753 & 69 & $\mathrm{~F}$ & Colon & Adenocarcinoma & T3N1M0 & 2 & III & malignant & + & 128 & + \\
\hline 754 & 76 & $\mathrm{~F}$ & Colon & Adenocarcinoma & T3N1M0 & 2 & III & malignant & + & 97 & + \\
\hline 755 & 76 & $\mathrm{~F}$ & Colon & Adenocarcinoma & T3N1M0 & 2 & III & malignant & + & 92 & + \\
\hline 756 & 30 & $\mathrm{~F}$ & Colon & Adenocarcinoma & T3N1M0 & 2 & III & malignant & + & 90 & + \\
\hline 757 & 30 & $\mathrm{~F}$ & Colon & Adenocarcinoma & T3N1M0 & 2 & III & malignant & + & 88 & + \\
\hline
\end{tabular}




\begin{tabular}{|c|c|c|c|c|c|c|c|c|c|c|c|}
\hline 758 & 66 & $\mathrm{~F}$ & Colon & Adenocarcinoma & T3N1M0 & 2 & III & malignant & + & 85 & + \\
\hline 759 & 66 & $\mathrm{~F}$ & Colon & Adenocarcinoma & T3N1M0 & 2 & III & malignant & + & 93 & + \\
\hline 760 & 42 & $\mathrm{~F}$ & Colon & Adenocarcinoma & T3N1M0 & 2 & III & malignant & + & 96 & + \\
\hline 761 & 42 & $\mathrm{~F}$ & Colon & Adenocarcinoma & T3N1M0 & 2 & III & malignant & + & 107 & + \\
\hline 762 & 65 & $\mathrm{~F}$ & Colon & Adenocarcinoma & T3N1M0 & 3 & III & malignant & + & 109 & + \\
\hline 763 & 65 & $\mathrm{~F}$ & Colon & Adenocarcinoma & T3N1M0 & 3 & III & malignant & + & 84 & + \\
\hline 764 & 60 & M & Colon & Adenocarcinoma & T3N1M0 & $2--3$ & III & malignant & + & 89 & + \\
\hline 765 & 60 & M & Colon & Adenocarcinoma (sparse) & T3N1M0 & $2--3$ & III & malignant & + & 96 & + \\
\hline 766 & 68 & M & Colon & Adenocarcinoma & $\mathrm{T} 4 \mathrm{~N} 1 \mathrm{M} 0$ & 3 & III & malignant & + & 163 & + \\
\hline 767 & 68 & M & Colon & Adenocarcinoma & $\mathrm{T} 4 \mathrm{~N} 1 \mathrm{M} 0$ & 3 & III & malignant & + & 152 & + \\
\hline 768 & 37 & M & Colon & Adenocarcinoma & T4N1M0 & 2 & III & malignant & + & 148 & + \\
\hline 769 & 37 & M & Colon & Adenocarcinoma & T4N1M0 & 2 & III & malignant & + & 139 & + \\
\hline 770 & 72 & $\mathrm{~F}$ & Colon & Adenocarcinoma & T4N1M0 & $2--3$ & III & malignant & + & 135 & + \\
\hline 771 & 72 & $\mathrm{~F}$ & Colon & Adenocarcinoma & $\mathrm{T} 4 \mathrm{~N} 1 \mathrm{M} 0$ & $2--3$ & III & malignant & + & 112 & + \\
\hline 772 & 24 & M & Colon & Mucinous adenocarcinoma & T3N2M0 & 3 & III & malignant & + & 118 & + \\
\hline 773 & 24 & M & Colon & Mucinous adenocarcinoma & T3N2M0 & 3 & III & malignant & + & 127 & + \\
\hline 774 & 60 & $\mathrm{~F}$ & Colon & Mucinous adenocarcinoma & T3N1M0 & 2 & III & malignant & + & 124 & + \\
\hline 775 & 60 & $\mathrm{~F}$ & Colon & Mucinous adenocarcinoma & T3N1M0 & 2 & III & malignant & + & 105 & + \\
\hline 776 & 42 & M & Colon & Mucinous adenocarcinoma (sparse) & T3N1M0 & 1 & III & malignant & + & 101 & + \\
\hline 777 & 42 & M & Colon & Mucinous adenocarcinoma (sparse) & T3N1M0 & 1 & III & malignant & + & 89 & + \\
\hline 778 & 59 & $\mathrm{~F}$ & Colon & Mucinous adenocarcinoma & T3N1M0 & 1 & III & malignant & + & 87 & + \\
\hline 779 & 59 & $\mathrm{~F}$ & Colon & Mucinous adenocarcinoma & T3N1M0 & 1 & III & malignant & + & 90 & + \\
\hline 780 & 48 & $\mathrm{~F}$ & Colon & Mucinous adenocarcinoma & T3N1M0 & 3 & III & malignant & + & 96 & + \\
\hline 781 & 48 & $\mathrm{~F}$ & Colon & Mucinous adenocarcinoma & T3N1M0 & 3 & III & malignant & + & 99 & + \\
\hline 782 & 45 & $\mathrm{~F}$ & Colon & Adenocarcinoma & T4N2M0 & 2 & III & malignant & + & 136 & + \\
\hline 783 & 45 & $\mathrm{~F}$ & Colon & Adenocarcinoma & T4N2M0 & 2 & III & malignant & + & 128 & + \\
\hline 784 & 52 & M & Colon & Adenocarcinoma & T3N1M0 & 2 & III & malignant & + & 109 & + \\
\hline 785 & 52 & M & Colon & Adenocarcinoma & T3N1M0 & 2 & III & malignant & + & 117 & + \\
\hline 786 & 42 & M & Colon & Adenocarcinoma & $\mathrm{T} 4 \mathrm{~N} 1 \mathrm{M} 0$ & 2 & III & malignant & + & 110 & + \\
\hline 787 & 42 & M & Colon & Adenocarcinoma & $\mathrm{T} 4 \mathrm{~N} 1 \mathrm{M} 0$ & 2 & III & malignant & + & 104 & + \\
\hline 788 & 52 & $M$ & Colon & Adenocarcinoma & $\mathrm{T} 4 \mathrm{~N} 1 \mathrm{M} 0$ & 2 & III & malignant & + & 98 & + \\
\hline 789 & 52 & M & Colon & Adenocarcinoma & T4N1M0 & 2 & III & malignant & + & 87 & + \\
\hline 790 & 47 & M & Colon & Adenocarcinoma & T4N1M0 & 2 & III & malignant & + & 84 & +++ \\
\hline 791 & 47 & M & Colon & Adenocarcinoma & $\mathrm{T} 4 \mathrm{~N} 1 \mathrm{M} 0$ & 2 & III & malignant & + & 93 & + \\
\hline 792 & 60 & $\mathrm{M}$ & Colon & Adenocarcinoma & T3N1M0 & 2 & III & malignant & + & 91 & + \\
\hline 793 & 60 & $M$ & Colon & Adenocarcinoma (sparse) & T3N1M0 & 2 & III & malignant & + & 98 & + \\
\hline
\end{tabular}




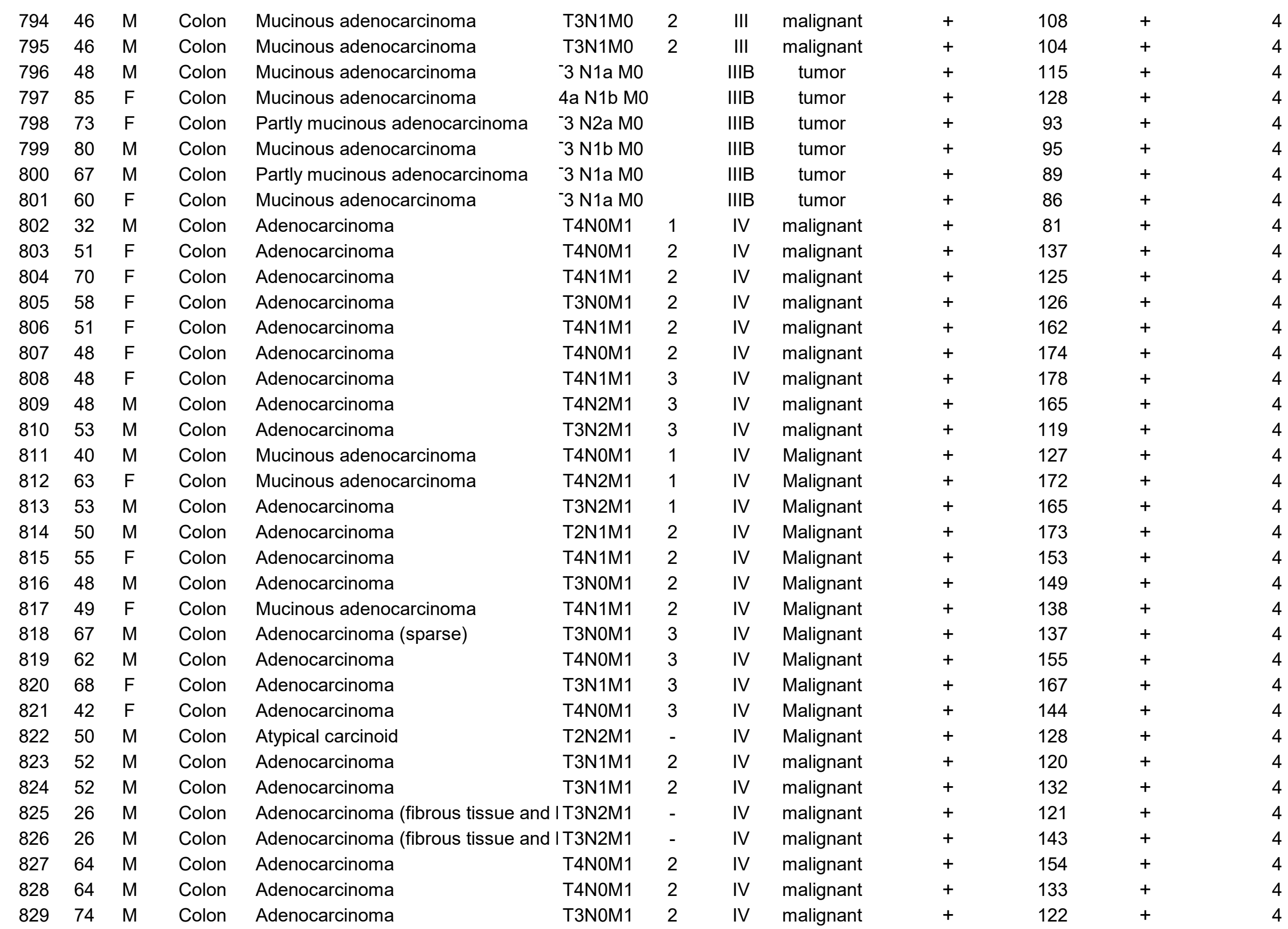




\begin{tabular}{|c|c|c|c|c|c|}
\hline 830 & 74 & $\mathrm{M}$ & Colon & Adenocarcinoma & T3N0M1 \\
\hline 831 & 56 & $\mathrm{M}$ & Colon & Adenocarcinoma & T3N0M1 \\
\hline 832 & 56 & M & Colon & Adenocarcinoma & T3N0M1 \\
\hline 833 & 77 & M & Colon & Mucinous adenocarcinoma & M1a \\
\hline 834 & 38 & $\mathrm{~F}$ & Colon & Partly mucinous adenocarcinoma & $\mathrm{N} 2 \mathrm{a} \mathrm{M} 1 \mathrm{a}$ \\
\hline 835 & 31 & $M$ & Colon & Mucinous adenocarcinoma & tb N1b M1a \\
\hline 836 & 70 & M & Colon & Mucinous adenocarcinoma & T3 M1b \\
\hline 837 & 70 & M & Colon & NAT (colon mucosal tissue ) & \\
\hline 838 & 53 & $\mathrm{~F}$ & Colon & Mucinous adenocarcinoma & M0 \\
\hline 839 & 53 & $\mathrm{~F}$ & Colon & NAT (colon mucosal tissue ) & \\
\hline 840 & 77 & M & Colon & NAT (colon mucosal tissue ) & \\
\hline 841 & 47 & M & Colon & NAT (colon mucosal tissue ) & \\
\hline 842 & 54 & $\mathrm{~F}$ & Colon & Partly mucinous adenocarcinoma & $\mathrm{T} 4 \mathrm{a} \mathrm{M0}$ \\
\hline 843 & 54 & $\mathrm{~F}$ & Colon & NAT (colon mucosal tissue ) & \\
\hline 844 & 79 & $\mathrm{~F}$ & Colon & Mucinous adenocarcinoma & T3 M0 \\
\hline 845 & 79 & $\mathrm{~F}$ & Colon & NAT (colon mucosal tissue ) & \\
\hline 846 & 48 & M & Colon & NAT (colon mucosal tissue ) & \\
\hline 847 & 85 & $\mathrm{~F}$ & Colon & NAT (colon mucosal tissue ) & \\
\hline 848 & 44 & M & Colon & NAT (colon mucosal tissue ) & \\
\hline 849 & 33 & M & Colon & NAT (colon mucosal tissue ) & \\
\hline 850 & 38 & $\mathrm{~F}$ & Colon & NAT (colon mucosal tissue ) & \\
\hline 851 & 31 & M & Colon & NAT (colon mucosal tissue ) & \\
\hline 852 & 75 & M & Colon & NAT (colon mucosal tissue ) & \\
\hline 853 & 70 & $\mathrm{M}$ & Colon & NAT (colon mucosal tissue ) & \\
\hline 854 & 70 & $\mathrm{~F}$ & Colon & NAT (colon mucosal tissue ) & \\
\hline 855 & 72 & M & Colon & NAT (colon mucosal tissue ) & \\
\hline 856 & 70 & M & Colon & NAT (colon mucosal tissue ) & \\
\hline 857 & 78 & $\mathrm{~F}$ & Colon & NAT (colon mucosal tissue ) & \\
\hline 858 & 73 & $\mathrm{~F}$ & Colon & NAT (colon mucosal tissue ) & \\
\hline 859 & 75 & M & Colon & NAT (colon mucosal tissue ) & \\
\hline 860 & 80 & M & Colon & NAT (colon mucosal tissue ) & \\
\hline 861 & 67 & M & Colon & NAT (colon mucosal tissue ) & \\
\hline 862 & 56 & $\mathrm{~F}$ & Colon & NAT (colon mucosal tissue ) & \\
\hline 863 & 40 & M & Colon & Partly mucinous adenocarcinoma & NO MO \\
\hline 864 & 40 & M & Colon & NAT (colon mucosal tissue ) & \\
\hline 865 & 69 & $\mathrm{M}$ & Colon & NAT (colon mucosal tissue ) & \\
\hline
\end{tabular}

$\begin{array}{cc}\text { IV } & \text { malignant } \\ \text { IV } & \text { malignant } \\ \text { IV } & \text { malignant } \\ \text { IVA } & \text { tumor } \\ \text { IVA } & \text { tumor } \\ \text { IVA } & \text { tumor } \\ \text { IVB } & \text { tumor } \\ & \\ & \text { tumor }\end{array}$




\begin{tabular}{|c|c|c|c|c|c|}
\hline 866 & 63 & $\mathrm{M}$ & Colon & Partly mucinous adenocarcinoma & NO MO \\
\hline 867 & 63 & M & Colon & NAT (colon mucosal tissue ) & \\
\hline 868 & 78 & $\mathrm{~F}$ & Colon & Little mucinous adenocarcinoma & NO MO \\
\hline 869 & 78 & $\mathrm{~F}$ & Colon & NAT (colon mucosal tissue ) & \\
\hline 870 & 57 & M & Colon & NAT (colon mucosal tissue ) & \\
\hline 871 & 72 & $M$ & Colon & Partly mucinous adenocarcinoma & NO MO \\
\hline 872 & 72 & $M$ & Colon & NAT (colon mucosal tissue ) & \\
\hline 873 & 60 & $\mathrm{~F}$ & Colon & NAT (colon mucosal tissue ) & \\
\hline 874 & 60 & $\mathrm{~F}$ & Colon & NAT (colon mucosal tissue ) & \\
\hline 875 & 27 & $M$ & Colon & Adenocarcinoma & T4NOMO \\
\hline 876 & 61 & M & Colon & Adenocarcinoma & T4NOMO \\
\hline 877 & 62 & $\mathrm{~F}$ & Colon & Adenocarcinoma & T4NOMO \\
\hline 878 & 55 & $M$ & Colon & Adenocarcinoma & T2NOMO \\
\hline 879 & 44 & M & Colon & Adenocarcinoma & T4NOMO \\
\hline 880 & 46 & $\mathrm{~F}$ & Colon & Adenocarcinoma & T3NOMO \\
\hline 881 & 68 & $\mathrm{~F}$ & Colon & Adenocarcinoma & T3NOMO \\
\hline 882 & 70 & $M$ & Colon & Adenocarcinoma & T3N1M0 \\
\hline 883 & 39 & M & Colon & Adenocarcinoma & T2NOMO \\
\hline 884 & 82 & M & Colon & Adenocarcinoma & T4NOMO \\
\hline 885 & 58 & $\mathrm{~F}$ & Colon & Adenocarcinoma & T3NOMO \\
\hline 886 & 73 & $M$ & Colon & Adenocarcinoma & T4NOMO \\
\hline 887 & 65 & $\mathrm{~F}$ & Colon & Adenocarcinoma & T3NOMO \\
\hline 888 & 49 & $M$ & Colon & Adenocarcinoma & T2NOMO \\
\hline 889 & 40 & M & Colon & Adenocarcinoma & T3NOMO \\
\hline 890 & 34 & $M$ & Colon & Adenocarcinoma & T3NOMO \\
\hline 891 & 61 & M & Colon & Mucinous adenocarcinoma & T3NOMO \\
\hline 892 & 59 & M & Colon & Adenocarcinoma & T2NOMO \\
\hline 893 & 68 & M & Colon & Adenocarcinoma & T3NOMO \\
\hline 894 & 62 & M & Colon & Adenocarcinoma & T3NOMO \\
\hline 895 & 60 & $\mathrm{~F}$ & Colon & Adenocarcinoma & T3NOMO \\
\hline 896 & 64 & $M$ & Colon & Adenocarcinoma & T3N1M0 \\
\hline 897 & 47 & $\mathrm{~F}$ & Colon & Adenocarcinoma & T2NOMO \\
\hline 898 & 75 & $M$ & Colon & Adenocarcinoma & T4NOMO \\
\hline 899 & 69 & $\mathrm{~F}$ & Colon & Adenocarcinoma & T2NOMO \\
\hline 900 & 55 & $\mathrm{~F}$ & Colon & Adenocarcinoma & T3NOMO \\
\hline 901 & 68 & $M$ & Colon & Adenocarcinoma & T4N0M0 \\
\hline
\end{tabular}

$\begin{array}{ll}\text { tumor } & + \\ \text { tumor } & + \\ & + \\ \text { tumor } & + \\ & + \\ \text { Malignant } & + \\ \text { Malignant } & + \\ \text { Malignant } & + \\ \text { Malignant } & + \\ \text { Malignant } & + \\ \text { Malignant } & + \\ \text { Malignant } & + \\ \text { Malignant } & + \\ \text { Malignant } & + \\ \text { Malignant } & + \\ \text { Malignant } & + \\ \text { Malignant } & + \\ \text { Malignant } & + \\ \text { Malignant } & + \\ \text { Malignant } & + \\ \text { Malignant } & + \\ \text { Malignant } & + \\ \text { Malignant } & + \\ \text { Malignant } & + \\ \text { Malignant } & + \\ \text { Malignant } & + \\ \text { Malignant } & + \\ \text { Malignant } & + \\ \text { Malignant } & + \\ \text { Malignant } & + \\ \text { Malignant } & + \\ \text { Malignant } & +\end{array}$

\begin{tabular}{|c|c|}
\hline 126 & + \\
\hline 134 & + \\
\hline 128 & + \\
\hline 145 & + \\
\hline 156 & + \\
\hline 178 & + \\
\hline 182 & + \\
\hline 180 & + \\
\hline 176 & + \\
\hline 84 & + \\
\hline 89 & + \\
\hline 94 & + \\
\hline 90 & + \\
\hline 83 & + \\
\hline 85 & + \\
\hline 91 & + \\
\hline 103 & + \\
\hline 87 & + \\
\hline 83 & + \\
\hline 89 & + \\
\hline 93 & + \\
\hline 91 & + \\
\hline 99 & + \\
\hline 102 & + \\
\hline 108 & + \\
\hline 83 & + \\
\hline 87 & + \\
\hline 98 & + \\
\hline 88 & + \\
\hline 91 & + \\
\hline 94 & + \\
\hline 85 & + \\
\hline 87 & + \\
\hline 96 & + \\
\hline 94 & + \\
\hline 89 & + \\
\hline
\end{tabular}




\begin{tabular}{|c|c|c|c|c|c|}
\hline 902 & 36 & M & Colon & Adenocarcinoma & T3NOMO \\
\hline 903 & 72 & $\mathrm{~F}$ & Colon & Adenocarcinoma & T4NOMO \\
\hline 904 & 47 & M & Colon & Adenocarcinoma & T2NOMO \\
\hline 905 & 32 & $\mathrm{~F}$ & Colon & Adenocarcinoma & T3NOMO \\
\hline 906 & 51 & M & Colon & Adenocarcinoma & T3NOMO \\
\hline 907 & 66 & M & Colon & Adenocarcinoma & T2NOMO \\
\hline 908 & 72 & M & Colon & Mucinous adenocarcinoma & T2NOMO \\
\hline 909 & 51 & $\mathrm{~F}$ & Colon & Mucinous adenocarcinoma & T4N0MO \\
\hline 910 & 58 & $\mathrm{~F}$ & Colon & Adenocarcinoma & T3NOMO \\
\hline 911 & 66 & M & Colon & Adenocarcinoma & T3NOMO \\
\hline 912 & 46 & M & Colon & Mucinous adenocarcinoma & $\mathrm{T} 2 \mathrm{~N} 1 \mathrm{MO}$ \\
\hline 913 & 67 & $\mathrm{~F}$ & Colon & Mucinous adenocarcinoma & T3NOMO \\
\hline 914 & 39 & M & Colon & Mucinous adenocarcinoma & T3NOMO \\
\hline 915 & 65 & $\mathrm{~F}$ & Colon & Mucinous adenocarcinoma & T3NOMO \\
\hline 916 & 74 & $\mathrm{~F}$ & Colon & Mucinous adenocarcinoma & T3NOMO \\
\hline 917 & 37 & $M$ & Colon & Mucinous adenocarcinoma & T3NOMO \\
\hline 918 & 58 & M & Colon & Mucinous adenocarcinoma & $\mathrm{T} 4 \mathrm{~N} 1 \mathrm{M} 1$ \\
\hline 919 & 74 & M & Colon & Mucinous adenocarcinoma & T4N0MO \\
\hline 920 & 59 & $\mathrm{~F}$ & Colon & Mucinous adenocarcinoma & T4N0MO \\
\hline 921 & 60 & M & Colon & Adenocarcinoma & T4NOMO \\
\hline 922 & 45 & $\mathrm{~F}$ & Colon & Adenocarcinoma & T3NOMO \\
\hline 923 & 68 & M & Colon & Adenocarcinoma & T4NOMO \\
\hline 924 & 43 & $\mathrm{~F}$ & Colon & Adenocarcinoma & T4NOMO \\
\hline 925 & 72 & M & Colon & Adenocarcinoma & T3NOMO \\
\hline 926 & 36 & M & Colon & Adenocarcinoma & T4NOMO \\
\hline 927 & 44 & $\mathrm{~F}$ & Colon & Adenocarcinoma & T4NOMO \\
\hline 928 & 37 & $M$ & Colon & Adenocarcinoma & $\mathrm{T} 4 \mathrm{~N} 1 \mathrm{M} 0$ \\
\hline 929 & 77 & $\mathrm{~F}$ & Colon & Adenocarcinoma & T2NOMO \\
\hline 930 & 52 & $\mathrm{~F}$ & Colon & Adenocarcinoma & T3NOMO \\
\hline 931 & 26 & M & Colon & Adenocarcinoma & T3N2M1 \\
\hline 932 & 55 & $\mathrm{~F}$ & Colon & Adenocarcinoma & T4NOMO \\
\hline 933 & 37 & $M$ & Colon & Adenocarcinoma & T2NOMO \\
\hline 934 & 73 & $M$ & Colon & Adenocarcinoma & T3NOMO \\
\hline 935 & 57 & $\mathrm{~F}$ & Colon & Adenocarcinoma & T3NOMO \\
\hline 936 & 57 & $M$ & Colon & Adenocarcinoma & T2NOMO \\
\hline 937 & 57 & $M$ & Colon & Adenocarcinoma & T3NOMO \\
\hline
\end{tabular}

$\begin{array}{ll}\text { Malignant } & + \\ \text { Malignant } & + \\ \text { Malignant } & + \\ \text { Malignant } & + \\ \text { Malignant } & + \\ \text { Malignant } & + \\ \text { Malignant } & + \\ \text { Malignant } & + \\ \text { Malignant } & + \\ \text { Malignant } & + \\ \text { Malignant } & + \\ \text { Malignant } & + \\ \text { Malignant } & + \\ \text { Malignant } & + \\ \text { Malignant } & + \\ \text { Malignant } & + \\ \text { Malignant } & + \\ \text { Malignant } & + \\ \text { Malignant } & + \\ \text { Malignant } & + \\ \text { Malignant } & + \\ \text { Malignant } & + \\ \text { Malignant } & + \\ \text { Malignant } & + \\ \text { Malignant } & + \\ \text { Malignant } & + \\ \text { Malignant } & + \\ \text { Malignant } & + \\ \text { Malignant } & + \\ \text { Malignant } & + \\ \text { Malignant } & + \\ \text { Malignant } & + \\ \text { Malignant } & + \\ \text { Malignant } & + \\ \text { Malignant } & + \\ \text { Malignant } & +\end{array}$

\begin{tabular}{|c|c|}
\hline & + \\
\hline $\begin{array}{l}90 \\
97\end{array}$ & + \\
\hline 103 & + \\
\hline 112 & + \\
\hline 109 & + \\
\hline 87 & + \\
\hline 89 & + \\
\hline 90 & + \\
\hline 85 & + \\
\hline 84 & + \\
\hline 67 & + \\
\hline 58 & + \\
\hline 43 & +++ \\
\hline 78 & + \\
\hline 79 & + \\
\hline 96 & + \\
\hline 103 & + \\
\hline 88 & + \\
\hline 85 & + \\
\hline 93 & + \\
\hline 90 & + \\
\hline 83 & +++ \\
\hline 89 & + \\
\hline 93 & + \\
\hline 92 & + \\
\hline 67 & + \\
\hline 54 & + \\
\hline 63 & + \\
\hline 83 & + \\
\hline 87 & + \\
\hline 90 & + \\
\hline 93 & + \\
\hline 89 & + \\
\hline 87 & + \\
\hline 94 & + \\
\hline 103 & + \\
\hline
\end{tabular}




$\begin{array}{lllll}938 & 59 & \text { M } & \text { Colon } & \text { Adenocarcinoma } \\ 939 & 57 & \mathrm{~F} & \text { Colon } & \text { Adenocarcinoma } \\ 940 & 61 & \mathrm{~F} & \text { Colon } & \text { Adenocarcinoma } \\ 941 & 63 & \mathrm{M} & \text { Colon } & \text { Adenocarcinoma } \\ 942 & 30 & \mathrm{M} & \text { Colon } & \text { Adenocarcinoma } \\ 943 & 77 & \mathrm{M} & \text { Colon } & \text { Adenocarcinoma } \\ 944 & 52 & \mathrm{M} & \text { Colon } & \text { Adenocarcinoma } \\ 945 & 62 & \mathrm{M} & \text { Colon } & \text { Adenocarcinoma } \\ 946 & 61 & \mathrm{M} & \text { Colon } & \text { Adenocarcinoma } \\ 947 & 54 & \mathrm{M} & \text { Colon } & \text { Adenocarcinoma } \\ 948 & 90 & \mathrm{M} & \text { Colon } & \text { Adenocarcinoma } \\ 949 & 70 & \mathrm{M} & \text { Colon } & \text { Adenocarcinoma } \\ 950 & 73 & \mathrm{M} & \text { Colon } & \text { Adenocarcinoma } \\ 951 & 72 & \mathrm{~F} & \text { Colon } & \text { Adenocarcinoma } \\ 952 & 74 & \mathrm{M} & \text { Colon } & \text { Adenocarcinoma } \\ 953 & 53 & \mathrm{M} & \text { Colon } & \text { Adenocarcinoma } \\ 954 & 54 & \mathrm{~F} & \text { Colon } & \text { Adenocarcinoma } \\ 955 & 62 & \mathrm{M} & \text { Colon } & \text { Adenocarcinoma } \\ 956 & 44 & \mathrm{M} & \text { Colon } & \text { Adenocarcinoma } \\ 957 & 60 & \mathrm{M} & \text { Colon } & \text { Adenocarcinoma } \\ 958 & 49 & \mathrm{~F} & \text { Colon } & \text { Adenocarcinoma } \\ 959 & 56 & \mathrm{~F} & \text { Colon } & \text { Adenocarcinoma } \\ 960 & 64 & \mathrm{~F} & \text { Colon } & \text { Adenocarcinoma } \\ 961 & 29 & \mathrm{~F} & \text { Colon } & \text { Adenocarcinoma } \\ 962 & 48 & \mathrm{~F} & \text { Colon } & \text { Adenocarcinoma } \\ 963 & 60 & \mathrm{M} & \text { Colon } & \text { Adenocarcinoma } \\ 964 & 47 & \mathrm{M} & \text { Colon } & \text { Adenocarcinoma } \\ 965 & 67 & \mathrm{~F} & \text { Colon } & \text { Adenocarcinoma } \\ 966 & 48 & \mathrm{M} & \text { Colon } & \text { Adenocarcinoma } \\ 967 & 64 & \mathrm{M} & \text { Colon } & \text { Adenocarcinoma } \\ 968 & 54 & \mathrm{M} & \text { Colon } & \text { Adenocarcinoma } \\ 969 & 75 & \mathrm{~F} & \text { Colon } & \text { Adenocarcinoma } \\ 970 & 68 & \mathrm{M} & \text { Colon } & \text { Adenocarcinoma } \\ 971 & 46 & \mathrm{M} & \text { Colon } & \text { Adenocarcinoma } \\ 972 & 78 & \mathrm{M} & \text { Colon } & \text { Adenocarcinoma } \\ 973 & 42 & \mathrm{~F} & \text { Colon } & \text { Adenocarcinoma }\end{array}$

$\begin{array}{ll}\text { T3NOM0 } & 2 \\ \text { T3NOM0 } & 2 \\ \text { T3NOM0 } & - \\ \text { T2NOM0 } & 2 \\ \text { T3NOM0 } & 2 \\ \text { T3NOM0 } & - \\ \text { T2NOM0 } & 3 \\ \text { T2NOM0 } & 2 \\ \text { T2NOM0 } & 2 \\ \text { T3NOM0 } & 1 \\ \text { T2NOM0 } & 1 \\ \text { T2NOM0 } & 1 \\ \text { T3NOM0 } & 1 \\ \text { T2NOM0 } & 1 \\ \text { T3NOM1 } & 1 \\ \text { T3NOM0 } & 1 \\ \text { T4NOM0 } & 2 \\ \text { T1NOM0 } & 1 \\ \text { T3NOM0 } & 1 \\ \text { T3NOM0 } & 1 \\ \text { T4NOM0 } & 2 \\ \text { T4NOM0 } & 2 \\ \text { T2NOM0 } & 1 \\ \text { T3NOM0 } & 2 \\ \text { T3NOM0 } & 2 \\ \text { T3NOM0 } & - \\ \text { T4N1M0 } & 2 \\ \text { T2NOM0 } & 1 \\ \text { T2NOM0 } & 1 \\ \text { T4NOM0 } & 1 \\ \text { T2NOM0 } & 2 \\ \text { T2NOM0 } & 2 \\ \text { T2NOM0 } & 2 \\ \text { T3NOM0 } & 2 \\ \text { T2NOM0 } & 2 \\ \text { T3NOM0 } & 2 \\ \end{array}$

$\begin{array}{ll}\text { Malignant } & + \\ \text { Malignant } & + \\ \text { Malignant } & + \\ \text { Malignant } & + \\ \text { Malignant } & + \\ \text { Malignant } & + \\ \text { Malignant } & + \\ \text { Malignant } & + \\ \text { Malignant } & + \\ \text { Malignant } & + \\ \text { Malignant } & + \\ \text { Malignant } & + \\ \text { Malignant } & + \\ \text { Malignant } & + \\ \text { Malignant } & + \\ \text { Malignant } & + \\ \text { Malignant } & + \\ \text { Malignant } & + \\ \text { Malignant } & + \\ \text { Malignant } & + \\ \text { Malignant } & + \\ \text { Malignant } & + \\ \text { Malignant } & + \\ \text { Malignant } & + \\ \text { Malignant } & + \\ \text { Malignant } & + \\ \text { Malignant } & + \\ \text { Malignant } & + \\ \text { Malignant } & + \\ \text { Malignant } & + \\ \text { Malignant } & + \\ \text { Malignant } & + \\ \text { Malignant } & + \\ \text { Malignant } & + \\ \text { Malignant } & + \\ \text { Malignant } & +\end{array}$

\begin{tabular}{|c|c|}
\hline 112 & + \\
\hline 98 & + \\
\hline 94 & + \\
\hline 86 & + \\
\hline 89 & + \\
\hline 81 & + \\
\hline 92 & + \\
\hline 83 & + \\
\hline 88 & + \\
\hline 87 & + \\
\hline 94 & + \\
\hline 103 & + \\
\hline 109 & + \\
\hline 117 & + \\
\hline 86 & + \\
\hline 83 & + \\
\hline 92 & + \\
\hline 90 & + \\
\hline 97 & + \\
\hline 89 & + \\
\hline 88 & + \\
\hline 108 & + \\
\hline 105 & + \\
\hline 115 & + \\
\hline 110 & + \\
\hline 121 & + \\
\hline 87 & + \\
\hline 89 & + \\
\hline 93 & + \\
\hline 97 & + \\
\hline 86 & + \\
\hline 107 & + \\
\hline 114 & + \\
\hline 123 & + \\
\hline 108 & + \\
\hline 105 & + \\
\hline
\end{tabular}




$\begin{array}{ccccc}974 & 52 & \mathrm{M} & \text { Colon } & \text { Adenocarcinoma } \\ 975 & 81 & \mathrm{M} & \text { Colon } & \text { Adenocarcinoma } \\ 976 & 32 & \mathrm{M} & \text { Colon } & \text { Adenocarcinoma } \\ 977 & 36 & \mathrm{~F} & \text { Colon } & \text { Adenocarcinoma } \\ 978 & 47 & \mathrm{M} & \text { Colon } & \text { Adenocarcinoma } \\ 979 & 43 & \mathrm{M} & \text { Colon } & \text { Adenocarcinoma } \\ 980 & 81 & \mathrm{M} & \text { Colon } & \text { Adenocarcinoma } \\ 981 & 63 & \mathrm{~F} & \text { Colon } & \text { Adenocarcinoma } \\ 982 & 60 & \mathrm{M} & \text { Colon } & \text { Adenocarcinoma } \\ 983 & 62 & \mathrm{~F} & \text { Colon } & \text { Adenocarcinoma } \\ 984 & 64 & \mathrm{M} & \text { Colon } & \text { Adenocarcinoma } \\ 985 & 65 & \mathrm{M} & \text { Colon } & \text { Adenocarcinoma } \\ 986 & 86 & \mathrm{M} & \text { Colon } & \text { Adenocarcinoma } \\ 987 & 48 & \mathrm{~F} & \text { Colon } & \text { Adenocarcinoma } \\ 988 & 44 & \mathrm{M} & \text { Colon } & \text { Adenocarcinoma } \\ 989 & 75 & \mathrm{M} & \text { Colon } & \text { Adenocarcinoma } \\ 990 & 60 & \mathrm{M} & \text { Colon } & \text { Adenocarcinoma } \\ 991 & 70 & \mathrm{~F} & \text { Colon } & \text { Adenocarcinoma } \\ 992 & 67 & \mathrm{M} & \text { Colon } & \text { Adenocarcinoma } \\ 993 & 57 & \mathrm{M} & \text { Colon } & \text { Adenocarcinoma } \\ 994 & 33 & \mathrm{~F} & \text { Colon } & \text { Adenocarcinoma } \\ 995 & 75 & \mathrm{M} & \text { Colon } & \text { Adenocarcinoma } \\ 996 & 31 & \mathrm{M} & \text { Colon } & \text { Adenocarcinoma } \\ 997 & 67 & \mathrm{~F} & \text { Colon } & \text { Adenocarcinoma } \\ 998 & 66 & \mathrm{~F} & \text { Colon } & \text { Adenocarcinoma } \\ 999 & 72 & \mathrm{M} & \text { Colon } & \text { Adenocarcinoma } \\ 1000 & 53 & \mathrm{~F} & \text { Colon } & \text { Adenocarcinoma } \\ 1001 & 76 & \mathrm{~F} & \text { Colon } & \text { Adenocarcinoma } \\ 1002 & 69 & \mathrm{M} & \text { Colon } & \text { Adenocarcinoma } \\ 1003 & 35 & \mathrm{~F} & \text { Colon } & \text { Adenocarcinoma } \\ 1004 & 60 & \mathrm{M} & \text { Colon } & \text { Adenocarcinoma } \\ 1005 & 62 & \mathrm{M} & \text { Colon } & \text { Adenocarcinoma } \\ 1006 & 48 & \mathrm{M} & \text { Colon } & \text { Adenocarcinoma } \\ 1007 & 55 & \mathrm{~F} & \text { Colon } & \text { Adenocarcinoma } \\ 1008 & 64 & \mathrm{~F} & \text { Colon } & \text { Adenocarcinoma } \\ 1009 & 58 & \mathrm{M} & \text { Colon } & \text { Adenocarcinoma }\end{array}$

$\begin{array}{ll}\text { T3NOMO } & 2 \\ \text { T2NOM0 } & 2 \\ \text { T3NOM0 } & - \\ \text { T3NOM0 } & 2 \\ \text { T3NOM0 } & - \\ \text { T3NOM0 } & 2 \\ \text { T2NOM0 } & 2 \\ \text { T4NOM0 } & 2 \\ \text { T3NOM0 } & 2 \\ \text { T3NOM0 } & 2 \\ \text { T2NOM0 } & 2 \\ \text { T3NOM0 } & 2 \\ \text { T4N1M0 } & 2 \\ \text { T4N1M0 } & 2 \\ \text { T4NOM0 } & 3 \\ \text { T4NOM0 } & 2 \\ \text { T3N1M0 } & 2 \\ \text { T3NOM0 } & 2 \\ \text { T4NOM0 } & 2 \\ \text { T3NOM0 } & 2 \\ \text { T3NOM0 } & 2 \\ \text { T4NOM0 } & 2 \\ \text { T4NOM0 } & 1 \\ \text { T3NOM0 } & 2 \\ \text { T2NOM0 } & 1 \\ \text { T3NOM0 } & 1 \\ \text { T3NOM0 } & 1 \\ \text { T2NOM0 } & 2 \\ \text { T4NOM0 } & 2 \\ \text { T3NOM0 } & 2 \\ \text { T4NOM0 } & 2 \\ \text { T4NOM0 } & 1 \\ \text { T2NOM0 } & 1 \\ \text { T4NOM0 } & 1 \\ \text { T2NOM0 } & 2 \\ \text { T2NOM0 } & - \\ & \end{array}$

$\begin{array}{ll}\text { Malignant } & + \\ \text { Malignant } & + \\ \text { Malignant } & + \\ \text { Malignant } & + \\ \text { Malignant } & + \\ \text { Malignant } & + \\ \text { Malignant } & + \\ \text { Malignant } & + \\ \text { Malignant } & + \\ \text { Malignant } & + \\ \text { Malignant } & + \\ \text { Malignant } & + \\ \text { Malignant } & + \\ \text { Malignant } & + \\ \text { Malignant } & + \\ \text { Malignant } & + \\ \text { Malignant } & + \\ \text { Malignant } & + \\ \text { Malignant } & + \\ \text { Malignant } & + \\ \text { Malignant } & + \\ \text { Malignant } & + \\ \text { Malignant } & + \\ \text { Malignant } & + \\ \text { Malignant } & + \\ \text { Malignant } & + \\ \text { Malignant } & + \\ \text { Malignant } & + \\ \text { Malignant } & + \\ \text { Malignant } & + \\ \text { Malignant } & + \\ \text { Malignant } & + \\ \text { Malignant } & + \\ \text { Malignant } & + \\ \text { Malignant } & + \\ \text { Malignant } & + \\ & +\end{array}$

\begin{tabular}{|c|c|}
\hline 82 & + \\
\hline 81 & + \\
\hline 93 & + \\
\hline 107 & + \\
\hline 88 & + \\
\hline 81 & + \\
\hline 93 & + \\
\hline 129 & + \\
\hline 121 & + \\
\hline 81 & + \\
\hline 83 & + \\
\hline 92 & + \\
\hline 98 & + \\
\hline 97 & + \\
\hline 109 & + \\
\hline 105 & + \\
\hline 89 & + \\
\hline 82 & + \\
\hline 94 & + \\
\hline 98 & + \\
\hline 103 & + \\
\hline 94 & + \\
\hline 87 & + \\
\hline 83 & + \\
\hline 92 & + \\
\hline 97 & + \\
\hline 83 & + \\
\hline 104 & + \\
\hline 109 & + \\
\hline 127 & + \\
\hline 135 & + \\
\hline 121 & + \\
\hline 101 & + \\
\hline 109 & + \\
\hline 97 & + \\
\hline 98 & + \\
\hline
\end{tabular}




\begin{tabular}{|c|c|c|c|c|c|}
\hline 1010 & 41 & $\mathrm{~F}$ & Colon & Adenocarcinoma & T2NOMO \\
\hline 1011 & 46 & $M$ & Colon & Adenocarcinoma & T3N0MO \\
\hline 1012 & 63 & $\mathrm{~F}$ & Colon & Adenocarcinoma & T3N0MO \\
\hline 1013 & 57 & $\mathrm{~F}$ & Colon & Adenocarcinoma & T3NOMO \\
\hline 1014 & 48 & $M$ & Colon & Adenocarcinoma & T3NOMO \\
\hline 1015 & 66 & M & Colon & Adenocarcinoma & T3NOMO \\
\hline 1016 & 66 & $M$ & Colon & Adenocarcinoma & T4NOMO \\
\hline 1017 & 64 & $\mathrm{~F}$ & Colon & Adenocarcinoma & T2NOMO \\
\hline 1018 & 77 & $\mathrm{~F}$ & Colon & Adenocarcinoma & T3N1M0 \\
\hline 1019 & 74 & $M$ & Colon & Adenocarcinoma & T3N0MO \\
\hline 1020 & 61 & $\mathrm{~F}$ & Colon & Adenocarcinoma & T2N1M0 \\
\hline 1021 & 64 & $M$ & Colon & Adenocarcinoma & T2NOMO \\
\hline 1022 & 45 & $M$ & Colon & Adenocarcinoma & T3NOMO \\
\hline 1023 & 50 & M & Colon & Adenocarcinoma & T3NOMO \\
\hline 1024 & 63 & $\mathrm{~F}$ & Colon & Adenocarcinoma & T3NOMO \\
\hline 1025 & 46 & $\mathrm{~F}$ & Colon & Adenocarcinoma & T2NOMO \\
\hline 1026 & 60 & M & Colon & Adenocarcinoma & T3NOMO \\
\hline 1027 & 62 & $\mathrm{~F}$ & Colon & Adenocarcinoma & T3NOMO \\
\hline 1028 & 80 & M & Colon & Adenocarcinoma & T2NOMO \\
\hline 1029 & 48 & $M$ & Colon & Adenocarcinoma & T2NOMO \\
\hline 1030 & 23 & M & Colon & Mucinous adenocarcinoma & T3N0MO \\
\hline 1031 & 46 & M & Colon & Mucinous adenocarcinoma & T3N1M0 \\
\hline 1032 & 60 & $\mathrm{~F}$ & Colon & Mucinous adenocarcinoma & T2NOMO \\
\hline 1033 & 74 & $\mathrm{~F}$ & Colon & Adenocarcinoma & T3NOMO \\
\hline 1034 & 59 & $\mathrm{~F}$ & Colon & Mucinous adenocarcinoma & T3N1M0 \\
\hline 1035 & 69 & $\mathrm{~F}$ & Colon & Adenocarcinoma & T4NOMO \\
\hline 1036 & 53 & M & Colon & Mucinous adenocarcinoma & T3NOMO \\
\hline 1037 & 51 & M & Colon & Adenocarcinoma & T2NOMO \\
\hline 1038 & 60 & M & Colon & Adenocarcinoma & T2NOMO \\
\hline 1039 & 56 & $\mathrm{~F}$ & Colon & Adenocarcinoma & T4NOMO \\
\hline 1040 & 56 & M & Colon & Adenocarcinoma & T3N0MO \\
\hline 1041 & 80 & $\mathrm{~F}$ & Colon & Adenocarcinoma & T2NOMO \\
\hline 1042 & 47 & M & Colon & Adenocarcinoma & T2NOMO \\
\hline 1043 & 68 & M & Colon & Adenocarcinoma & T4NOMO \\
\hline 1044 & 78 & M & Colon & Adenocarcinoma & T3NOMO \\
\hline 1045 & 50 & $M$ & Colon & Adenocarcinoma & T4NOMO \\
\hline
\end{tabular}

\begin{tabular}{|c|c|c|c|}
\hline Malignant & + & 81 & + \\
\hline Malignant & + & 87 & + \\
\hline Malignant & + & 86 & + \\
\hline Malignant & + & 105 & + \\
\hline Malignant & + & 118 & + \\
\hline Malignant & + & 137 & + \\
\hline Malignant & + & 129 & + \\
\hline Malignant & + & 103 & + \\
\hline Malignant & + & 84 & + \\
\hline Malignant & + & 98 & + \\
\hline Malignant & + & 87 & + \\
\hline Malignant & + & 94 & + \\
\hline Malignant & + & 103 & + \\
\hline Malignant & + & 89 & + \\
\hline Malignant & + & 87 & + \\
\hline Malignant & + & 96 & + \\
\hline Malignant & + & 104 & + \\
\hline Malignant & + & 108 & + \\
\hline Malignant & + & 117 & + \\
\hline Malignant & + & 124 & + \\
\hline Malignant & + & 132 & + \\
\hline Malignant & + & 143 & + \\
\hline Malignant & + & 88 & + \\
\hline Malignant & + & 89 & + \\
\hline Malignant & + & 93 & + \\
\hline Malignant & + & 94 & + \\
\hline Malignant & + & 90 & + \\
\hline Malignant & + & 102 & + \\
\hline Malignant & + & 104 & + \\
\hline Malignant & + & 107 & + \\
\hline Malignant & + & 83 & + \\
\hline Malignant & + & 87 & + \\
\hline Malignant & + & 91 & + \\
\hline Malignant & + & 94 & + \\
\hline Malignant & + & 128 & + \\
\hline Malignant & + & 116 & + \\
\hline
\end{tabular}




$\begin{array}{lllll}1046 & 47 & \text { M } & \text { Colon } & \text { Mucinous adenocarcinoma } \\ 1047 & 46 & \text { M } & \text { Colon } & \text { Adenocarcinoma } \\ 1048 & 52 & \text { M } & \text { Colon } & \text { Adenocarcinoma } \\ 1049 & 50 & \text { M } & \text { Colon } & \text { Adenocarcinoma } \\ 1050 & 68 & \text { M } & \text { Colon } & \text { Mucinous adenocarcinoma } \\ 1051 & 37 & \text { M } & \text { Colon } & \text { Adenocarcinoma } \\ 1052 & 67 & \text { F } & \text { Colon } & \text { Adenocarcinoma } \\ 1053 & 48 & \text { M } & \text { Colon } & \text { Adenocarcinoma } \\ 1054 & 38 & \text { M } & \text { Colon } & \text { Adenocarcinoma } \\ 1055 & 46 & \text { M } & \text { Colon } & \text { Adenocarcinoma } \\ 1056 & 77 & \text { M } & \text { Colon } & \text { Adenocarcinoma } \\ 1057 & 39 & \text { M } & \text { Colon } & \text { Adenocarcinoma } \\ 1058 & 35 & \text { M } & \text { Colon } & \text { Adenocarcinoma } \\ 1059 & 65 & \text { F } & \text { Colon } & \text { Adenocarcinoma } \\ 1060 & 74 & \text { M } & \text { Colon } & \text { Adenocarcinoma } \\ 1061 & 56 & \text { M } & \text { Colon } & \text { Adenocarcinoma } \\ 1062 & 50 & \text { F } & \text { Colon } & \text { Adenocarcinoma } \\ 1063 & 35 & \text { M } & \text { Colon } & \text { Signet ring cell carcinoma } \\ 1064 & 31 & \text { M } & \text { Colon } & \text { Adenocarcinoma } \\ 1065 & 59 & \text { M } & \text { Colon } & \text { Mucinous adenocarcinoma } \\ 1066 & 51 & \text { M } & \text { Colon } & \text { Cancer adjacent colon tissue } \\ 1067 & 68 & \text { M } & \text { Colon } & \text { Cancer adjacent colon tissue } \\ 1068 & 52 & \text { M } & \text { Colon } & \text { Cancer adjacent colon tissue } \\ 1069 & 35 & \text { M } & \text { Colon } & \text { Cancer adjacent colon tissue } \\ 1070 & & \text { M } & \text { Colon } & \text { Colon tissue } \\ 1071 & & \text { M } & \text { Colon } & \text { Colon tissue } \\ 1072 & & \text { M } & \text { Colon } & \text { Colon tissue } \\ 1073 & & \text { M } & \text { Colon } & \text { Colon tissue } \\ 1074 & & \text { M } & \text { Colon } & \text { Colon tissue } \\ 1075 & & \text { M } & \text { Colon } & \text { Colon tissue } \\ & & & & \\ 1075 & & \end{array}$

$\begin{array}{cc}\text { T3NOM0 } & 2 \\ \text { T4NOM0 } & 3 \\ \text { T3NOM0 } & 3 \\ \text { T4NOM0 } & 2 \\ \text { T3NOM0 } & 2 \\ \text { T4N1M1 } & 2 \\ \text { T3NOM0 } & - \\ \text { T2NOM0 } & 3 \\ \text { T4N1M0 } & 3 \\ \text { T3NOM0 } & 3 \\ \text { T4NOM0 } & 3 \\ \text { T2NOM0 } & 3 \\ \text { T2NOM0 } & 3 \\ \text { T2NOM0 } & 3 \\ \text { T3NOM0 } & 3 \\ \text { T3NOM1 } & 3 \\ \text { T2NOM0 } & 3 \\ \text { T3NOM0 } & - \\ \text { T4NOM0 } & 3 \\ \text { T3NOM0 } & * \\ - & - \\ - & - \\ - & - \\ - & - \\ & \\ & \\ & \\ & \\ & \\ & \\ & \\ & \\ & \end{array}$

$\begin{array}{cc}\text { Malignant } & + \\ \text { Malignant } & + \\ \text { Malignant } & + \\ \text { Malignant } & + \\ \text { Malignant } & + \\ \text { Malignant } & + \\ \text { Malignant } & + \\ \text { Malignant } & + \\ \text { Malignant } & + \\ \text { Malignant } & + \\ \text { Malignant } & + \\ \text { Malignant } & + \\ \text { Malignant } & + \\ \text { Malignant } & + \\ \text { Malignant } & + \\ \text { Malignant } & + \\ \text { Malignant } & + \\ \text { Malignant } & + \\ \text { Malignant } & + \\ \text { Malignant } & + \\ \text { NAT } & + \\ \text { NAT } & + \\ \text { NAT } & + \\ \text { NAT } & + \\ \text { Normal } & - \\ \text { Normal } & - \\ \text { Normal } & - \\ \text { Normal } & - \\ \text { Normal } & - \\ \text { Normal } & -\end{array}$

$\begin{array}{lll}109 & + & 4 \\ 129 & + & 4 \\ 135 & + & 4 \\ 142 & + & 4 \\ 144 & + & 4 \\ 138 & + & 4 \\ 127 & + & 4 \\ 126 & + & 4 \\ 138 & + & 4 \\ 87 & + & 4 \\ 94 & + & 4 \\ 97 & + & 4 \\ 81 & + & 4 \\ 104 & + & 4 \\ 107 & + & 4 \\ 67 & + & 3 \\ 85 & + & 4 \\ 108 & + & 4 \\ 127 & + & 4 \\ 106 & + & 4 \\ 134 & + & 4 \\ 156 & + & 4 \\ 167 & + & 4 \\ 103 & + & 4\end{array}$

